\title{
Structure Changes in Nephila Dragline: The Influence of Temperature, Humidity and Mechanical Load
}

\author{
Dissertation \\ zur Erlangung des Doktorgrades \\ der Mathematisch-Naturwissenschaftlichen Fakultäten \\ der Georg-August-Universität zu Göttingen
}

vorgelegt von

Anja Glišović

aus Lübeck

Göttingen 2007 
D7

Referent: Prof. Dr. Tim Salditt

Koreferent: Prof. Dr. Annette Zippelius

Tag der mündlichen Prüfung: 4. Mai 2007 
"If at first you don't succeed, try, try and try again."

Robert the Bruce, King of Scots (1274 - 1329) 



\section{Contents}

1 Introduction 2

2 Nephila Dragline - Composition, Structure and Models 4

2.1 Proteins ........................... 5

2.2 Dragline Silk - From Primary Structure to Fiber . . . . . . . . . . . . 8

2.3 Stress-Strain Curves . . . . . . . . . . . . . . . . . . . . . . 12

2.3.1 Hysteresis . . . . . . . . . . . . . . . . 15

2.4 Dragline's Mechanical Properties . . . . . . . . . . . . . . . . . . . . 17

2.5 Models . . . . . . . . . . . . . . . . . . . . . . . 19

2.5.1 Hierarchical Chain Model . . . . . . . . . . . . . . . . . . . 19

2.5.2 Molecular Modeling of Spider Silk Elasticity . . . . . . . . . 22

3 X-Ray Diffraction, Instrumentation and Data Analysis 26

3.1 Fiber Diffraction . . . . . . . . . . . . . . . . 30

3.2 1D-Patterns and Fiber Bundles . . . . . . . . . . . 33

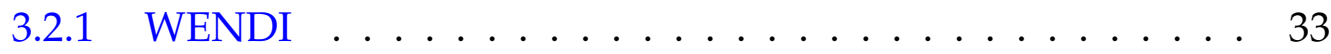

3.3 2D-Patterns - Single Fiber Diffraction . . . . . . . . . . . . . . 34

3.3.1 Microfocus Beamline ID13 equipped with KB-Optics . . . . 34

3.3.2 Microfocus Beamline ID13 equipped with CRL-Optics . . . 35

3.3.3 Raw Data Treatment and Calibration . . . . . . . . . . . . 35

3.4 X-Ray Data Analysis . . . . . . . . . . . . . . . . . 38

3.5 LEX 810 High Resolution Extensometer . . . . . . . . . . . . . 42

3.5.1 Humidity Chamber . . . . . . . . . . . . . . 43

4 Forced Silking and Samples Preparation 44

4.1 Degumming .......................... 45

4.2 Sample Preparation . . . . . . . . . . . . . . . . 45

5 Dragline - Humidity, Hysteresis and Relaxation 48

5.1 Influence of Humidity . . . . . . . . . . . . . . . . . . . . . 48

5.2 Hysteresis . . . . . . . . . . . . . . . . . 50

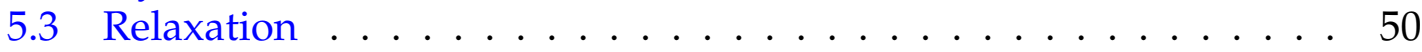

6 Scattering Simulation $\quad \mathbf{5 4}$

6.1 Simulation Program cBay . . . . . . . . . . . . . . . . . . . 55

6.2 Alanine Crystallites . . . . . . . . . . . . . . . . 56 
7 Thermal Changes in Dragline Fiber Bundles $\quad 60$

7.1 Samples, Measurement and Data Analysis . . . . . . . . . . . . . . . . . 60

7.2 Results ............................. 61

7.2.1 Lattice constants . . . . . . . . . . . . . . . . 6 62 62

7.2.2 Peak Intensity $\mathrm{I}(\mathrm{T})$ and Peak Area $\mathrm{A}(\mathrm{T}) \ldots \ldots 2$

7.2.3 Size of the Crystallites . . . . . . . . . . . . . . . . 64

7.3 Summary and Discussion . . . . . . . . . . . . . 65

8 Single Fiber Diffraction - The Influence of Strain $\quad 72$

8.1 Results ....................... 73

8.1.1 Angular Width and Crystallite Orientation . . . . . . . . . 73

8.1.2 Radial Width and Crystallite Size . . . . . . . . . . . . . . . 74

8.1.3 Peak Position and Lattice Constants . . . . . . . . . . . . . . 75

8.1.4 Amorphous Halo . . . . . . . . . . . . . . . . . 75

8.2 Discussion .......................... 76

9 Single Fiber Diffraction - The Effect of High Humidities and Strain 86

9.1 Influence of Humidity on the Structure at Low Strains . . . . . . . . 87

9.2 Immersed Fibers under Strain . . . . . . . . . . . . . . . . . . . . 93

9.2.1 Angular Width and Crystallite Orientation . . . . . . . . 93

9.2.2 Radial Width and Crystallite Size . . . . . . . . . . . . . . . . 93

9.2.3 Peak Position and Lattice Constants . . . . . . . . . . . . . . 93

9.2.4 Amorphous Halo . . . . . . . . . . . . . . . . . . . . . 94

9.3 Discussion . . . . . . . . . . . . . . . . . . 94

$\begin{array}{ll}10 \text { Summary } & 100\end{array}$

$\begin{array}{ll}\text { Abbreviations } & 102\end{array}$

$\begin{array}{ll}\text { Bibliography } & 104\end{array}$

A Small Angle X-Ray Scattering of Spider Silk 112

$\begin{array}{ll}\text { B Primary Structures } & \mathbf{1 1 6}\end{array}$

B.1 Nephila clavipes . . . . . . . . . . . . . . . . . . . . 1116

B.2 Nephila madagascariensis. . . . . . . . . . . . . . . . . . . . . . 118

B.3 Nephila senegalensis . . . . . . . . . . . . . . . . . . 119

C Fit2D Macros $\quad \mathbf{1 2 0}$

C.1 Equatorial, Meridional and Arc Slices . . . . . . . . . . . . . . 120

C.2 Off-Axis Slices . . . . . . . . . . . . . . . . . . . . . 133

C.3 Cake Integration . . . . . . . . . . . . . . . . . . 139

$\begin{array}{ll}\text { D List of Publications } & 146\end{array}$

$\begin{array}{lll}\text { E Acknowledgement } & 148\end{array}$

$\begin{array}{ll}\text { Lebenslauf } & \mathbf{1 5 0}\end{array}$ 


\section{Chapter 1}

\section{Introduction}

Important periods of mankind such as Stone Age, Bronze Age, or Iron Age have been named after materials. Modern times are marked by the use of a wide variety of mostly synthetic materials such as for example polymers. A general understanding of their functional properties is essential for our technological progress. In many cases biomaterials can compete with synthetic materials and beat them in sustainability, recyclability and biodegradability. Also they are synthesized at ambient conditions with only water as solvent. In respect to the rising ecological and economical issues, the characterization and understanding of the properties and the formation of biomaterials have become extremely important, as nature can inspire very useful inventions if only the underlying principles are understood. For example, George de Mestral invented velcro after observing burrs clinging to his dog's fur.

In recent years spider silk has attracted much interest as a fascinating biomaterial. Dragline fibers exceed nylon in extensibility and its tensile strength is near that of steel. This combination is highly unusual and hardly be mimicked by manmade fibers. Also the origin of these astonishing mechanical properties is yet not completely understood. Such knowledge is of fundamental importance for the synthesis, improvement and design of biomimetic, spider silk inspired polymers and custom made artificial spider silk $[1 ; 2 ; 3]$.

The correlation between molecular and mesoscopic structure and the mechanical properties was already found to be of great importance in biomaterials like bone, tendons and wood, where evolution has also accomplished outstanding performance by complex hierarchical structures and self assembly. Therefore it is reasonable to assume a similar correlation for spider silk which is under adaptation and optimization by evolution for more than 380 million years now [4]. In order to reveal this relation a wide variety of techniques is being used to characterize the chemical, mechanical, thermal and structural properties of spider silk. Among others the study of structure changes in dragline bundles performed by Grubb and Jelinski [5; 6] and the works of Riekel and Vollrath [7; 8] on single fiber diffraction mark the starting point for the spider silk project at the Institut für Röntgenphysik.

The goal of this thesis is to provide a more detailed insight into the correlation between spider silk structure and its mechanical properties. To this end, the influence of thermal and mechanical load as well as the influence of humidity were 
studied. The humidity and temperature controlled environments for the samples had to be adapted to fit the experimental needs. The use of single fibers also increased the experimental requirements significantly $[9 ; 10]$ but was essential to unambiguously link the structural changes to the applied mechanical load. The feasibility of combined single fiber X-ray diffraction, force-strain and humidity controlled experiments was established to obtain previously unaccessible information.

In this work special emphasis was given to the crystalline components of spider silk, due to their good accessibility by X-rays. The crystallites were characterized in respect to their response to humidity, strain and temperature. A possible mechanism for the fiber's intrinsic energy dissipation is proposed based on these results, as well as an explanation for the recovery of spider silk after immersion in water. Furthermore, the hysteresis effects (the 'extention memory') can be explained. Finally, the fiber's glass transition at high temperatures has been studied, along with the thermal denaturation process.

Following this introduction, chapter 2 presents composition, structure, mechanical properties and theoretical models of spider silk, as can be found in the literature. Furthermore it covers the basics of tensile studies and proteins. Fiber X-ray diffraction is briefly discussed in chapter 3 together with the details on instrumentation and data treatment. The art of keeping Nephila spiders and obtaining silk from them is described in chapter 4 . Chapter 5 is devoted to the mechanical properties, and chapter 6 to the simulation of the scattering distribution. The next three chapters' (7 - 9) emphasis is on the actual experiments and cover thermally induced changes in spider silk, as well as strain induced changes as observed by single fiber X-ray diffraction, both under ambient and humid conditions. In the final chapter 10 the results are summarized. 


\section{Chapter 2}

\section{Nephila Dragline - Composition, Structure, Models}

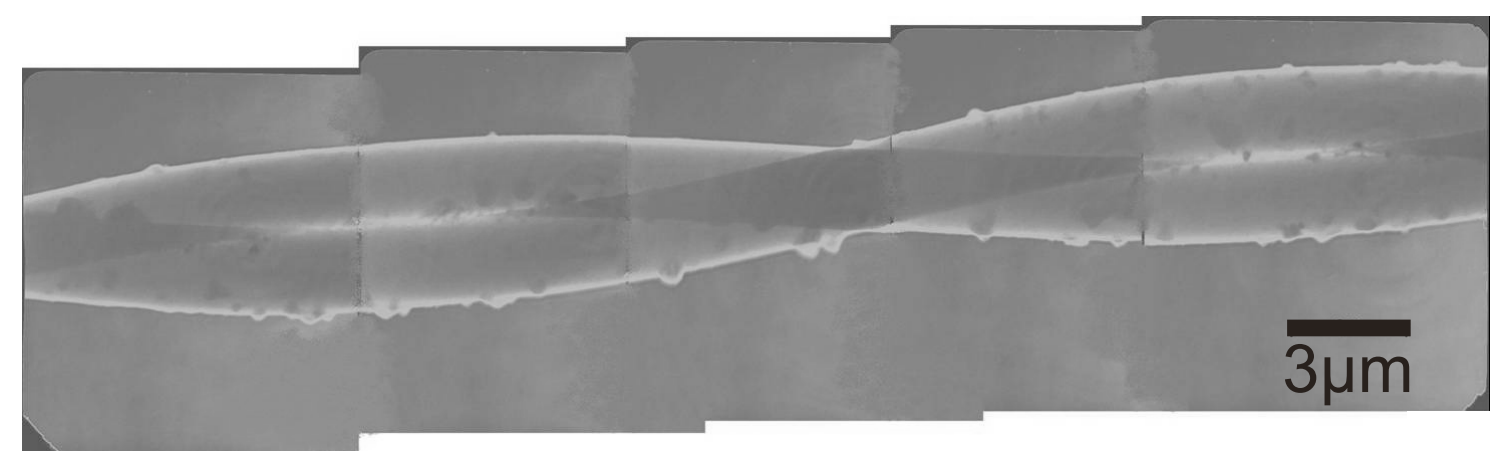

Figure 2.1: Transmission X-ray microscopy image of a Araneus MAS double fiber. The fibers are twisted around each other and flatten at the contact area [11; 12].

The genus Nephila has become the standard genus for research on spider silk due to the remarkable properties of their dragline, a body length of $4-5 \mathrm{~cm}$, a pacific character and easy keeping, see chapter 4.

Orb-weaving spiders like Nephila have up to seven different silk types at hand $[4 ; 13 ; 14]$. Every silk variety has its typical application and is produced and stored in a special gland, see figure 2.2. Each silk gland comprises a lumen and a duct to the external spinnerets. The silk proteins are secreted by the gland cells' endoplasmatic reticulum in form of fluid spinning dope, an aqueous protein solution. While passing through the duct the spinning dope undergoes a phase transition from liquid to solid fiber induced by shear [15; 16; 17] and a $p H$-gradient in the duct $[18 ; 19 ; 20]$. The spinning speed has a great influence on the structure and the mechanical properties of dragline and silkworm silk, a higher spinning rate leads to an increased toughness $[21 ; 22 ; 23 ; 24]$. The spider controls the silk flow by muscular valves at the duct's end.

The orb web's frame is built from dragline silk which is produced in the spider's major ampullate gland and also referred to as MAS or MA silk. The dragline also acts as the spider's life line, catching her safely after a free fall of several centimeters, when the spider drops from her web in moments of danger. 


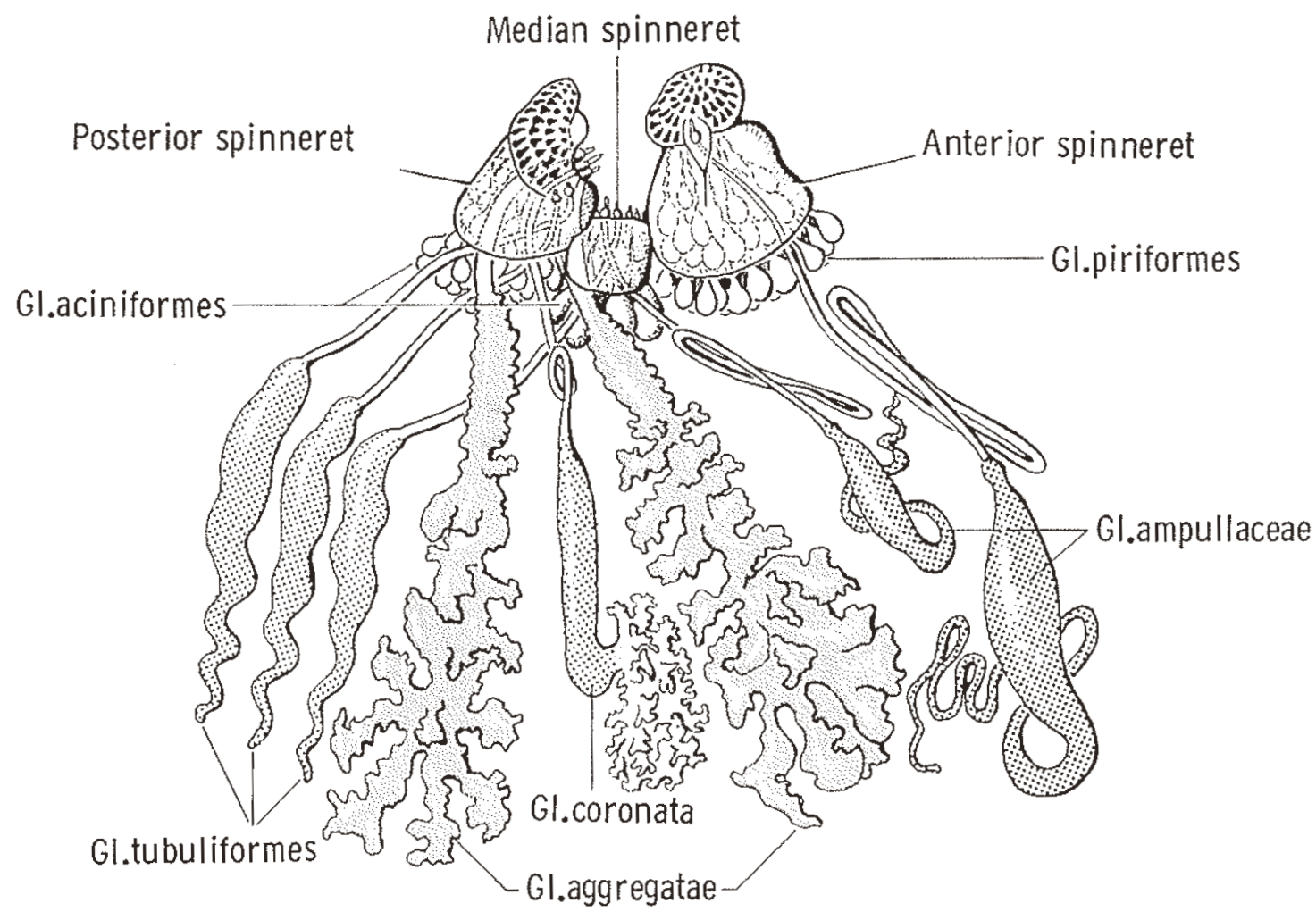

Figure 2.2: Spinning apparatus and silk glands of Nephila madagascariensis, from [25] after [13]. Gl. aciniformes - egg sac's outer wall and sperm web, Gl. tubuliformes - cocoon silk, Gl. coronata - axial thread of sticky spiral, Gl. aggregatae - glue of sticky spiral, Gl. ampullaceae - dragline, frame thread and Gl. piriformes - attachement disk.

\subsection{Proteins}

As all proteins silk consists of amino acids, dragline predominately of glycine and alanine [26; 27]. Amino acids (aa) are small molecules in which an carbon atom $\left(C_{\alpha}\right)$ connects an amine group and a carboxyl group. There are twenty natural amino acids which are used by cells in protein biosynthesis. The aa varieties differ by the side chain (R) attached to the $C_{\alpha}$-atom. The simplest amino acid (glycine) has a hydrogen atom as side chain while the second simplest aa (alanine) has a methyl group, more sophisticated side chains, like hydrocarbon chains with aromatic rings, are also possible. Two amino acids can be connected by a peptide bond between amine and carboxyl group, see figure 2.3. A peptide bond is not a classical covalent bond. The double bond characteristic of the $C O$ group is shared with the $C N$ bond (normally a single bond). The energy of such a resonant structure is higher than that of a normal covalent bond.

More than one hundered amino acids connected by peptide bonds are called a protein. The aa sequence (primary structure) is unique for every kind of protein. Primary structures of proteins are available online in protein data banks like ExPASy [28]. For the tested dragline silk of the three Nephila species, Nephila sene- 


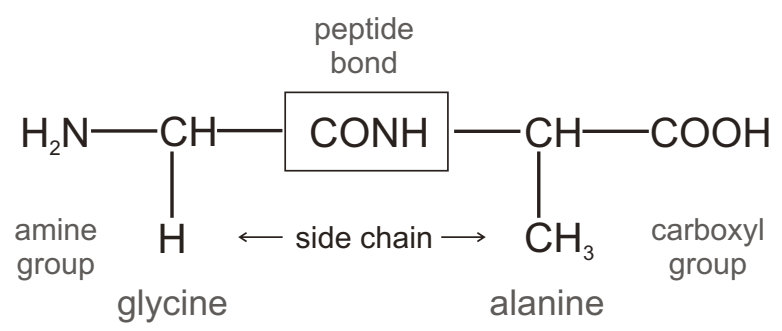

Figure 2.3: Schematic of an dipeptide consisting of glycine and alanine. The peptide bond is formed between the molecules' carboyl group $(-\mathrm{COOH})$ and amine group $\left(-\mathrm{NH}_{2}\right)$ by a dehydration synthesis reaction in which a water molecule is released: $\mathrm{COOH}+\mathrm{H}_{2} \mathrm{~N}=\mathrm{CONH}+\mathrm{H}_{2} \mathrm{O}$.

galensis (NS), Nephila clavipes (NC) and Nephila madagascariensis (NM), they are also listed in appendix B.

A protein achieves the most energy efficient configuration (secondary structure) by rotations around the dihedral angles $(\Phi, \Psi)$, see figure 2.4. The secondary structure is stabilized by hydrogen bonds between amino acids which approached each other during folding and depends strongly on the side chain's properties. When ever a number of subsequent amino acids has the same dihedral angles a regular secondary structure occurs, e.g. helices, $\beta$-turns or $\beta$-pleated sheets. $\beta$-Sheet strands have nearly the straightest possible conformation. Theoretically the maximal length of a dipeptide is $7.2 \AA$ [29], the length of a $\beta$-sheet dipeptide is $6.95 \AA$. The corresponding dihedral angles are $\Phi=40^{\circ}$ and $\Psi=215^{\circ}$ [30].

The arrangement of a protein's secondary structure elements is called tertiary structure. For the arrangement of $\beta$-sheet strands parallel and an anti-parallel (read from amine group to carboxyl group) conformations are possible, stabilized by hydrogen bonds between adjacent protein strands, see figure 2.5. Layers of parallel and anti-parallel $\beta$-sheets stacked on top of each other form a $\beta$-sheet crystallites, as shown in figure 2.6 for two layers. In $\beta$-sheet crystallites secondary and tertiary structure merge. They can connect different protein strands and therefore stabilize a material. 


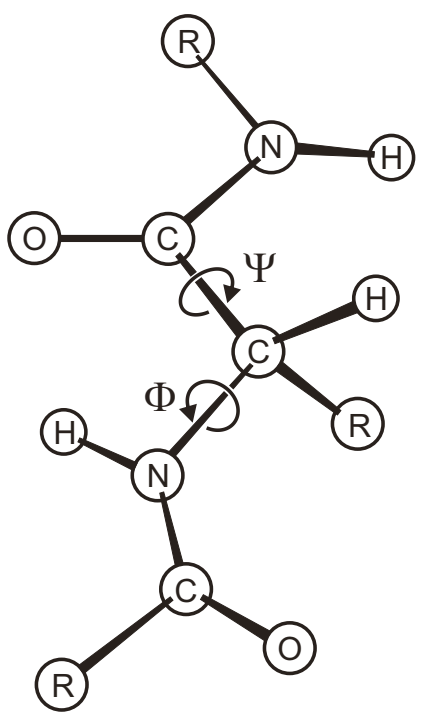

Figure 2.4: Schematic of a dipeptide. $R$ indicates a side chain. The molecule can rotate around the dihedral angels $\Phi$ and $\Psi$ to achieve the most energy efficient conformation. This process is also referred to as folding.

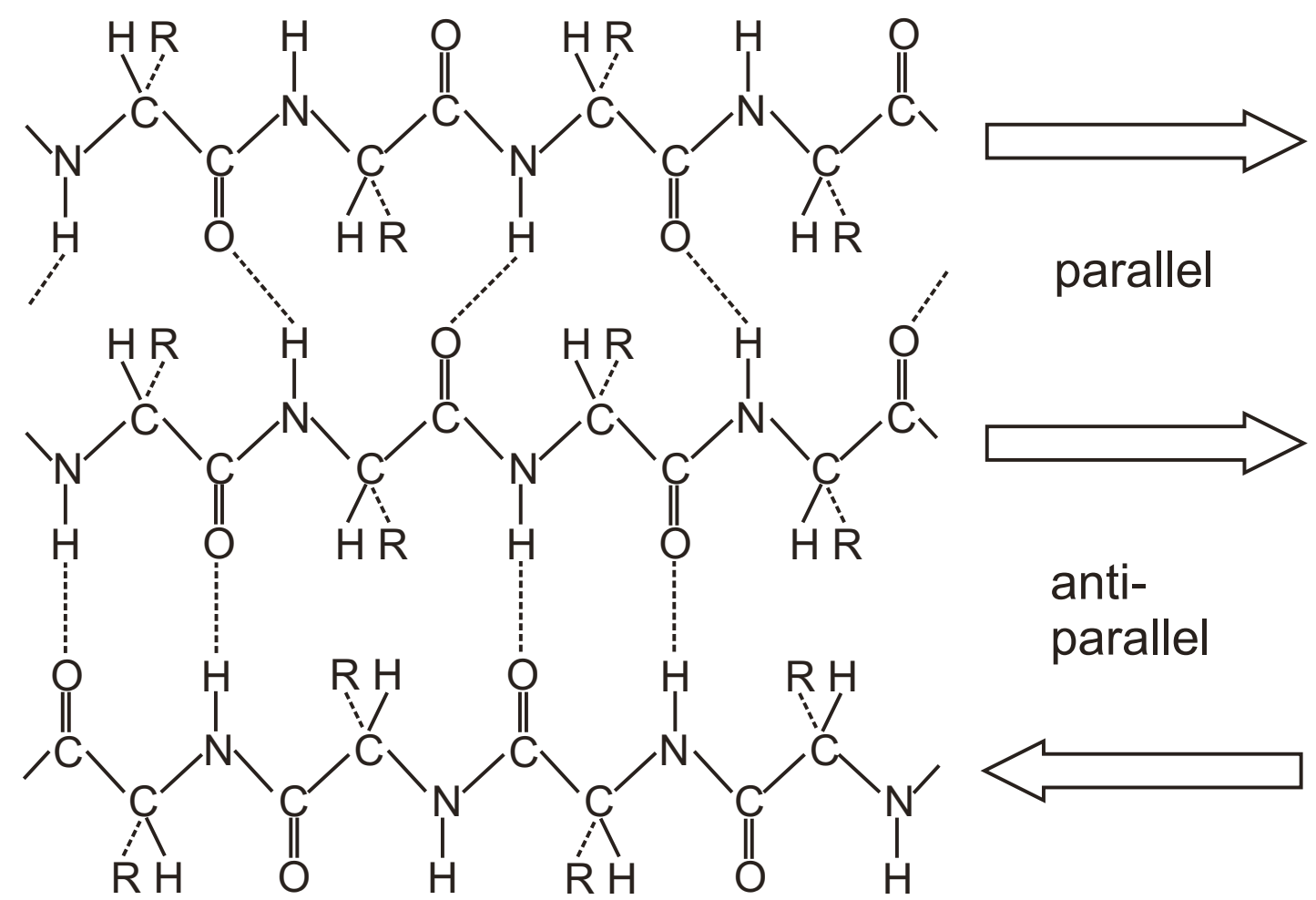

Figure 2.5: The $y z$-plane of a parallel and an anti-paralle arrangement of $\beta$-sheet strands. The strands are connected by hydrogen-bonds (dotted lines) in the yz-plane. In parallel conformation adjacent $\beta$-sheet strands run in the same direction (read from $N$-terminus to $C$-terminus) while directions alternate for the anti-parallel case. 


\subsection{Dragline Silk - From Primary Structure to Fiber}

Dragline silk consists of two proteins: Spidroin I [31] and Spidroin II [32]. Nephila Spidroins are highly repetitive, consisting of a periodic arrangement of similar amino acid motives ended by a none structured tail [24; 33]. E.g. NC Spidroin 1 is built from 747 amino acids with 25 motive repeats and Spidroin 2 from 627 aa with 15 motive repeats. A motive consists of a glycine rich block of 20-30 aa and a 5-8 aa alanine block. Therefore both Spidroins are diblock copolymers.

During the phase transition from liquid spinning dope to fiber the shorter alanine blocks aggregate in $\beta$-sheet crystallites [34] while the glycine rich parts from an amorphous matrix in which the crystallites are embedded. The secondary structure of the amorphous matrix is partly under discussion [35; 36]. NMR experiments, in which the signal from spider silk was compared with simulated signals of different secondary structures, propose 310 -helices as the predominate structure element [36]. On the other hand FTIR measurements indicate a mixture of random coil, $\beta$-turns and helices [37;38]. Observed with X-rays these parts of a dragline fiber give rise to an amorphous halo with a mean distance of the amino acids of approximately $3.5 \AA$. The ratio of crystalline fraction to amorphous fraction varies between 3:7 to 2:8 [5]. Own measurements of NC dragline lie with $25 \%$ crystallinity well within these values [12]. Often, he crystalline components are considered to be inactive cross links connecting different protein-strands and as a passive filler material reinforcing the amorphous matrix $[34 ; 39 ; 40]$.

By wide angle X-ray diffraction (WAXS) the $\beta$-sheets of all three Nephila species investigated have been classified to be anti-parallel [41], see figure 2.5 and to belong to the Warwicker System 3 [42; 43]. Accordingly, these types of anti-parallel $\beta$-pleated sheets have a pseudo orthorhombic unit cell [41] with $10.6 \AA \times 9.44 \AA$ $\times 6.95 \AA$ [42]. To fix the coordinate system:

$x$-axis Axis along the amino acid side chains; lattice constant $a=10.6 \AA$

$y$-axis Axis in the direction of the H-bonds; lattice constant $b=9.44 \AA$

$z$-axis Axis along the covalent peptide bonds; lattice constant $c=6.95 \AA$

A schematic representation of a comparable anti-parallel $\beta$-sheet is given in figure 2.6. The crystallites' $z$-axis is well-aligned along the fiber axis while $x$ - and $y$-axis are randomly distributed, see figure 2.7. Therefore it is also a nematic elastomer [44]. The size of the crystallites varies slightly for all three species. Typically the an average crystallite of NS is a little bigger than those of NM and NC. The values of all our measurements on Nephila draglines (single fiber and bundle), correspond to a mean crystallite for all three species with the dimensions of $53 \AA \times 47 \AA \times 60 \AA$, in all three symmetry axis, respectively. Bigger but less periodic crystallites were also suggest but could not experimentally verified yet [35]. Nevertheless the alanine blocks are with a max. length of $27.8 \AA$ too small to form the $\beta$-sheet crystallites alone. Most likely the alanine blocks will not be arranged exactly next to each other but shifted [45]. In such an arrangement the crystallites are dominated by the alanine blocks.

With the average size of the crystallites it is possible to estimate the minimal 
number of different Spidroin strands which form a crystallite. Approximately $5 \times 5$ unit cells form the $x y$-face of a $\beta$-sheet crystallite. On the surface of each unit cell four amino acids are arranged, so one hundred protein strands form a single crystallite. With maximal 25 repeating motives in a single Spidroin 1 strand this means that minimum four protein strands are necessary to build up a single $\beta$-sheet crystallite. A real $\beta$-sheet crystallite will be formed by more protein strands due to the fact that Spidroin 1 and Spidroin 2 have equal portion in the fiber and that the crystallites have to be interconnected for a stable fiber.

The packing of the crystallites is quite close [46; 47]. The mean distance between two crystallites along the fiber axis was found by Small Angle X-ray Scattering (SAXS) and Neutron Scattering to vary between $70 \AA$ - $80 \AA$ [6; 7; 48]. The crystallite's mean spacing perpendicular to the fiber axis could not be determined experimentally yet. But calculations based upon a crystalline fraction of $20 \%-30$ $\%$ and the given crystallite size of $53 \AA \times 47 \AA \times 60 \AA$ lead to a perpendicular mean distance of $50 \AA-60 \AA$, see appendix A.

A main issue about dragline silk's structure is the existence of another structural level between the arrangement of the crystallites and the macroscopic fiber. Some groups suggest a microfibrillar substructure [49] which is verified for fibers from the silkworm Bombyx mori (BM) [50]. Our own experiments performed by X-ray transmission microscopy on whole fibers and slices suggest that there is no such microfibrillar structure [11]. The diameter of Nephila dragline fibers range from $4 \mu \mathrm{m}$ for NC to $9 \mu \mathrm{m}$ for NS and depends on the spider's size and species. Due to the symmetrical arrangement of the two MA glands along the spider's abdominal center-line, dragline threads a spider uses are in double fiber conformation, as shown in figure 2.1. 


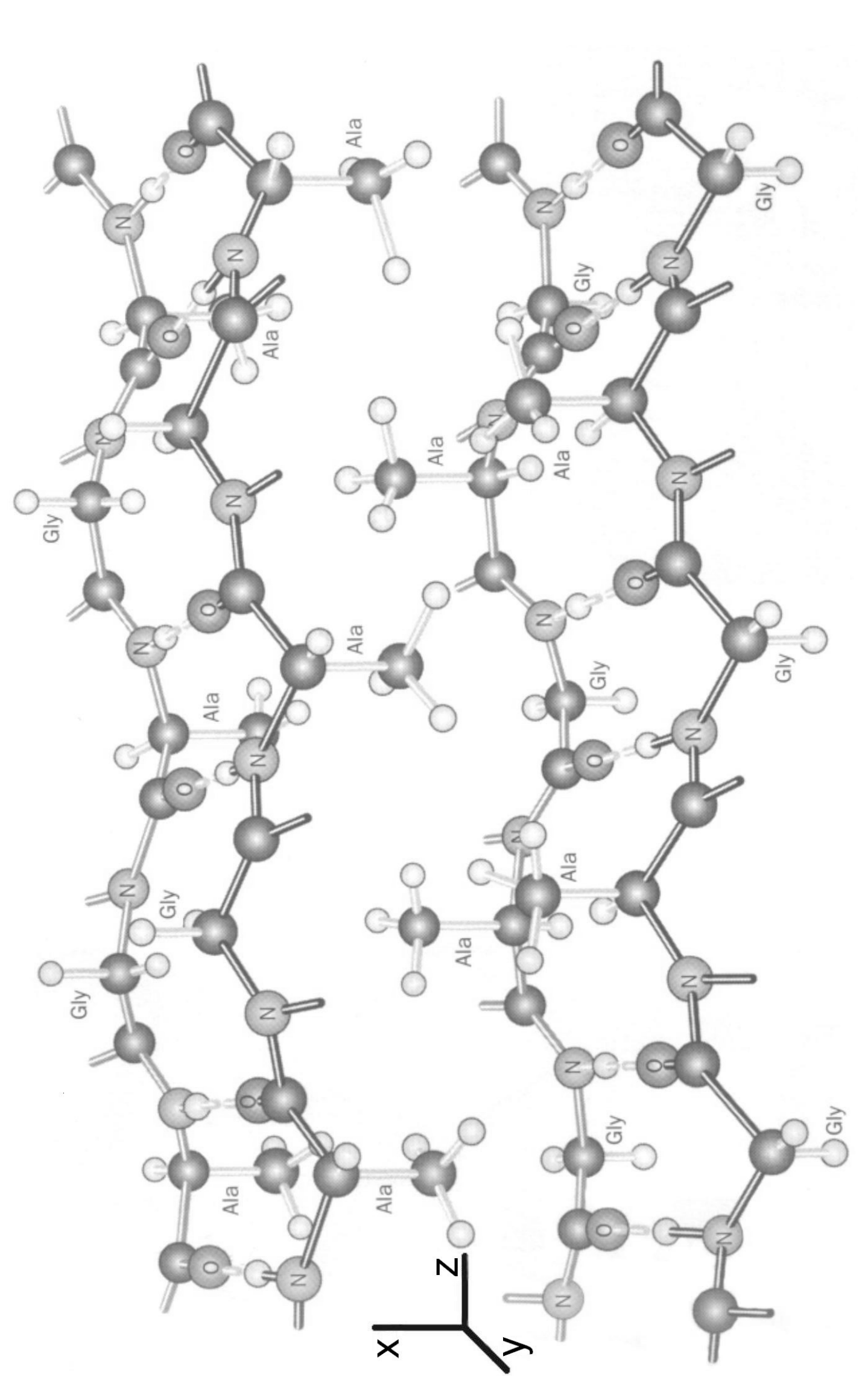

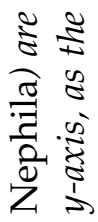

文芒

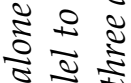

₹

है

से है

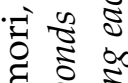

दे ड

ต

온

욜

릉

辛

ㅎํㄹำ

돌ำ.

क

돌

3.

की

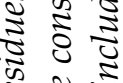

ญ.

₹

雯

运 8

की

0 공

उ.

ะ

के के

d.

है

$\times$ 도ㅇㅛㅛ

ह ํํㄹำ ว. 辛芒 जै को نे 류 率寒 


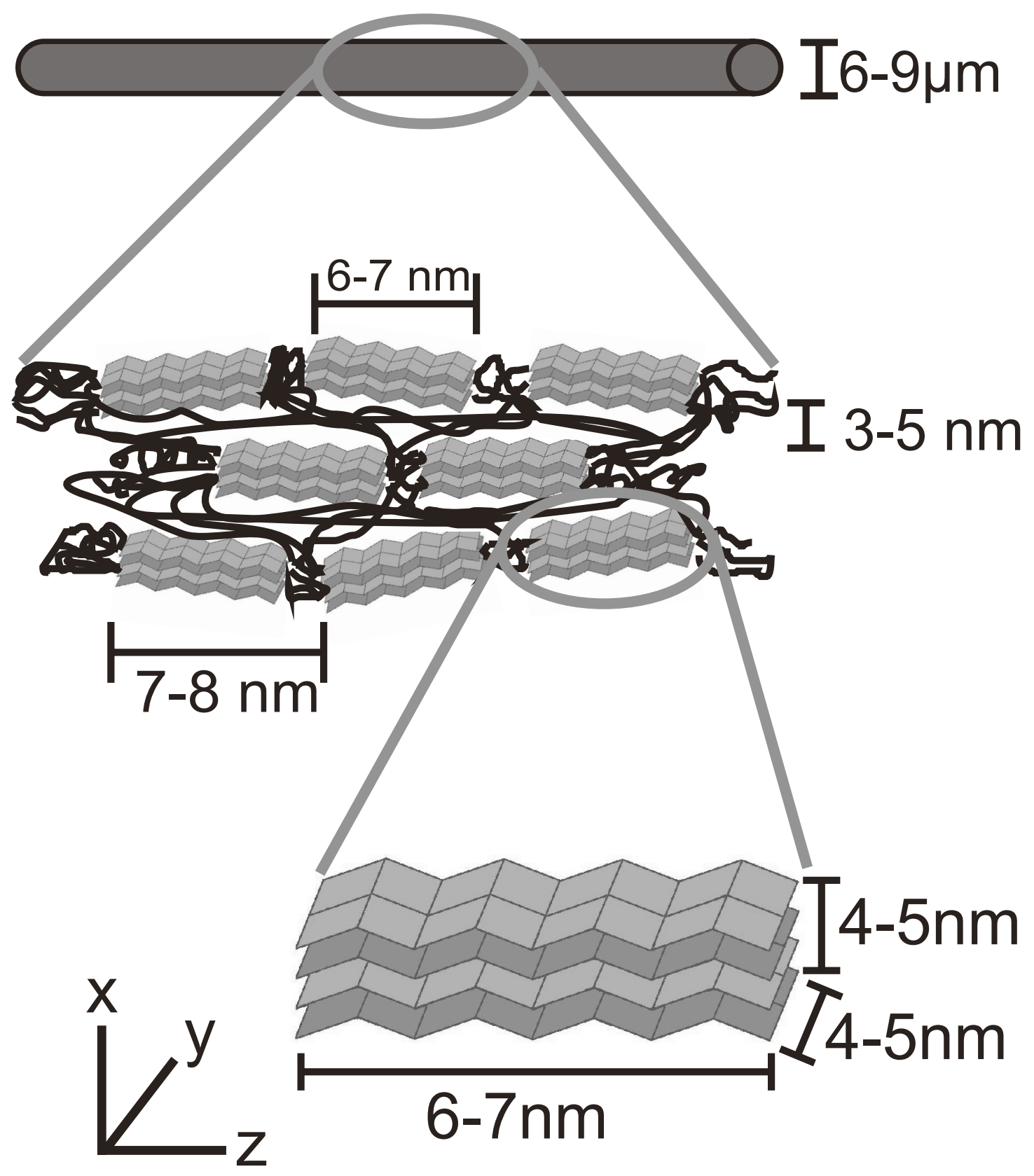

Figure 2.7: Structure of dragline silk: On micrometer scale the fiber, on nanometer scale amorphous matrix and $\beta$-sheet crystallites. 


\subsection{Stress-Strain Curves}

The standard method for tensile studies is the stress-strain measurement or, without the normalization by the fiber diameter, force-strain measurement in which the force is recorded versus elongation. Sometimes such curves are also referred to as $\varepsilon-\sigma$-curves or $\varepsilon$ - $\sigma$-diagrams with $\varepsilon=$ strain and $\sigma=$ stress. They characterize the sample's response to an applied tensile strain $\varepsilon$ and allow the determination of important information such as elastic modulus, breaking strain $\sigma_{\max }$, breaking force, toughness (total energy per volume until breaking point) and the amount of energy dissipation. Figure 2.8 shows three materials which represent an extremely stiff material (steel), a very extensible material (rubber) and an intermediate, so-called viscoelastic, material (perlon / polyamide 6). The stress-strain curves of Nephila dragline at approximately $50 \%$ relative humidity $(\mathbf{R H})$ is also shown. Spider silk lies in between the two extremes and is therefore also a viscoelastic material. The unusual form of the stress-strain curve with two nearly linear regions makes the difference to polyamide 6 . From such simple stress-strain curves the following properties can directly be retrieved:

Initial elastic modulus $\mathcal{E}$ : Slope of the initial linear region; $\sigma=\mathcal{E} \mathcal{E}$; Unit $P a$

Yield point: Normally the stress at which a material begins to plastically deform. For spider silk the point of the curve's main inflection [39].

Tensile strength: Breaking stress $\sigma_{\max }$ at which the fiber tears.

Extensibility: Breaking strain $\varepsilon_{\max }$ at which the fiber tears.

Toughness: Energy per unit volume a material can absorb before failure, represented by the integral of stress $\sigma$ over strain $\sigma$ which corresponds to the area under the curve. Unit $\mathrm{J} / \mathrm{m}^{3}$.

They are also shown in figure 2.9. Stress-strain measurements can be performed with or without gauge force $F_{G}$. Without a gauge force the measurement starts immediately. The stress-strain curves exhibit an initial region where the sample is not completely stretched and the force is nearly zero, see the region $0 \% \leq \varepsilon \leq 4 \%$ in figure 2.12, page 18. A gauge force causes the measurement to start only when the preset force threshold is crossed. Therefore the measurements do not start at zero but with an offset which is also used to define $l_{0}$ for the calculation of the relative elongation $\varepsilon=\left[\left(l-l_{0}\right) / l_{0}\right] \cdot 100$, see figure 2.10. Normally this offset is negligible but in hysteresis measurements it causes a shift in subsequent curves. These errors can be corrected either by linear regression or renormalization. For dragline silk a gauge force of $F_{G}=1.5 \mathrm{mN}$ proved itself. 


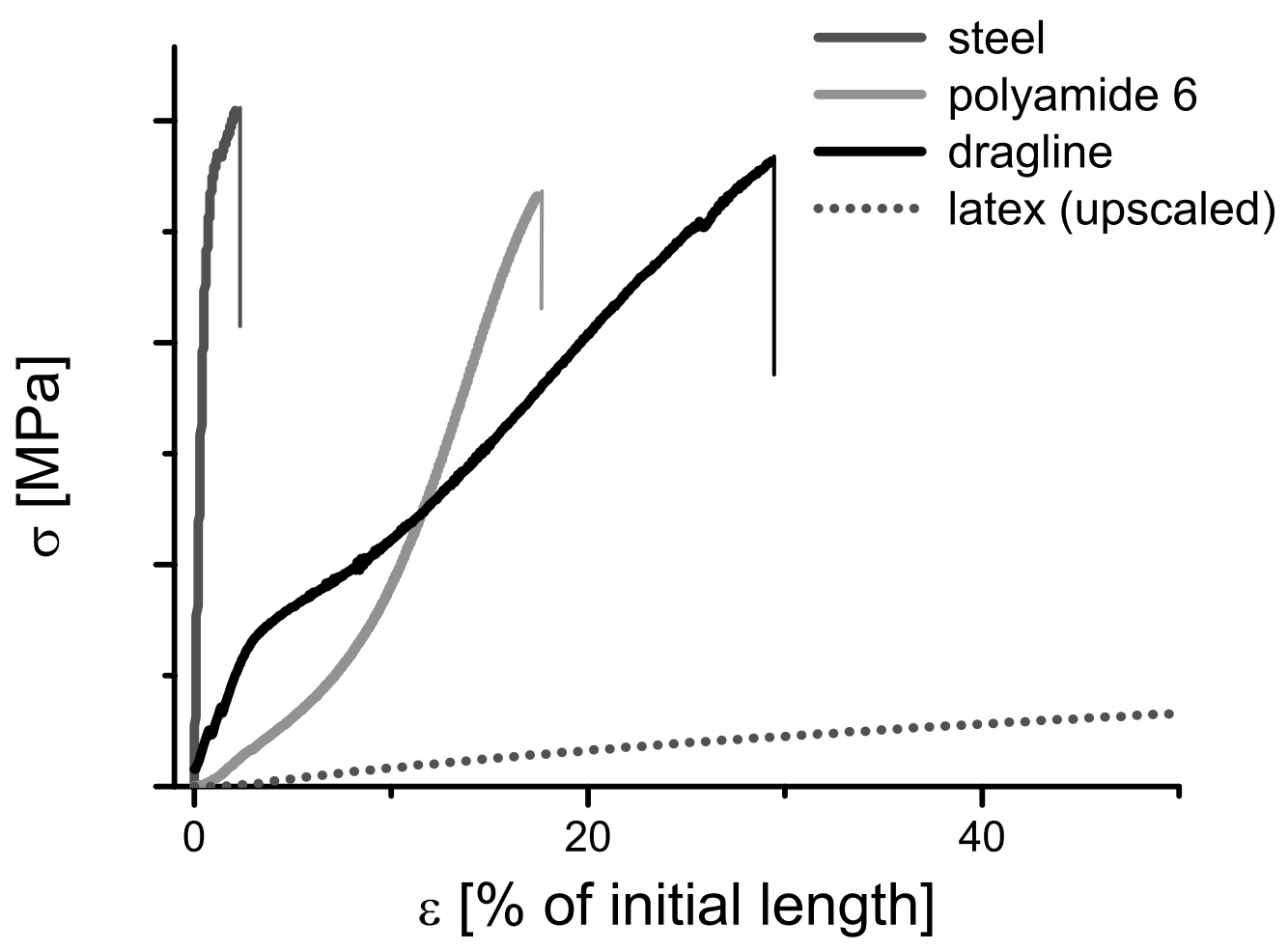

Figure 2.8: Stress-strain curves of steel, polyamide 6 (perlon), latex and NS dragline. Steel, polyamide 6 and dragline have a comparable maximum strain but different initial elastic moduli (slopes of the initial linear regimes), see also table 2.1. The area under the curve which corresponds to the energy stored in the fiber, is maximal for dragline. The stress-strain curve of latex is upscaled otherwise it would be too close to the $x$-axis. Also only the beginning of the latex stress-strain curve is shown. Latex can be stretched up to $800 \%$ of its initial length. 


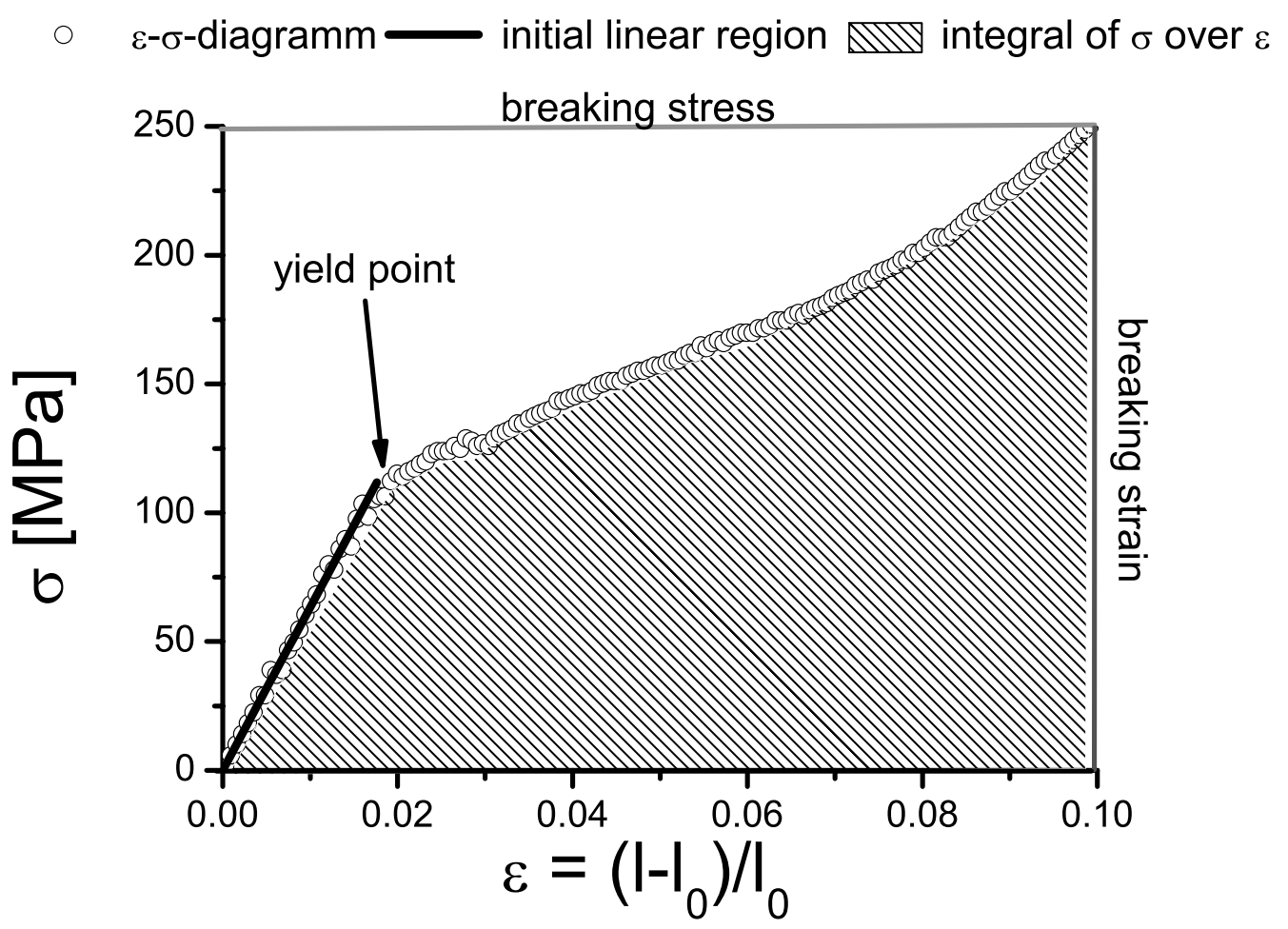

Figure 2.9: Main properties which can be retrieved from a simple stress-strain measurement shown exemplary on NS dragline. Initial elastic modulus = slope of the initial linear region. Yield point $=$ position where the two linear regimes merge into each other. Tensile strength = breaking stress $\sigma_{\max }$. Extensibility $=$ breaking strain $\varepsilon_{\max }$. Toughness (total energy per volume till breaking point $)=$ area under the curve. 


\subsubsection{Hysteresis}

The area under a force-strain curve resembles the integral of force over elongation and therefore the stretching energy. Partly the stretching energy is stored in the fiber, partly it is dissipated. The percentage of energy dissipation is measured by an advanced type of stress-strain experiments in which not only the fiber's response to elongation but also to reduced extension is recorded. Such measurements are called hysteresis measurements or just hysteresis and consist of two curves, see figure 2.10. One for increasing strain, called ascent and one for decreasing strain (descent). While the area under the ascent curve resembles the total stretching energy necessary, the area under the descent gives the energy elastically stored in the fiber. The dissipated energy then is the area between these two curves. For Nephila dragline the dissipated energy is around $68 \%$ of the total stretching energy [52; 53] and approximately $32 \%$ is elastically stored. Incidentally, this is the reason for the term viscoelastic, "viscous" like a fluid that dissipates energy and "elastic" for energy storage. Subsequent hysteresis measurements of the same sample indicate whether deformation and energy loss in the fiber are due to reversible or irreversible processes. No differences in subsequent hysteresis cycles indicate reversible processes, see figure 2.11, while significant changes indicate irreversible processes. Depending on the material this is normally true for the first two to five cycles after which fatigue failure occurs.

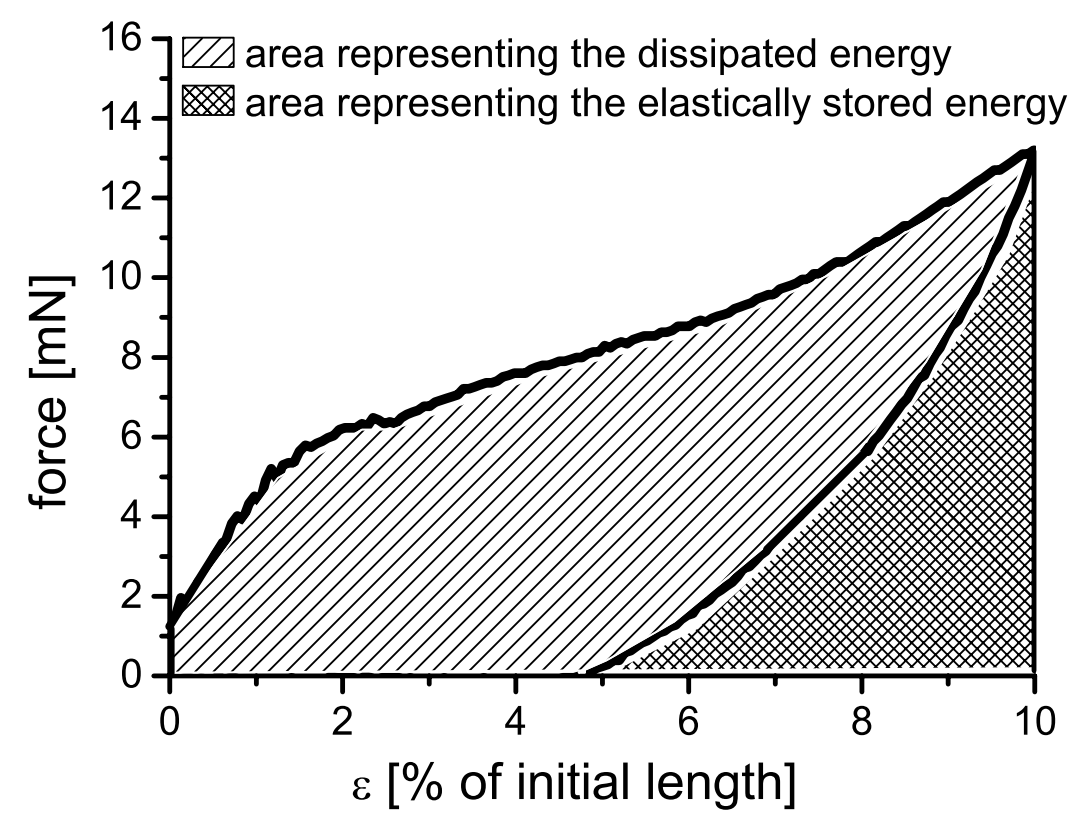

Figure 2.10: A typical hysteresis curve for NS dragline. The area under the ascending curve corresponds to the total energy necessary to stretch the fiber, the area under the descending curve to the energy elastically stored in the fiber (checkered). The differences between the two areas correspond to the dissipated energy (streaked). 


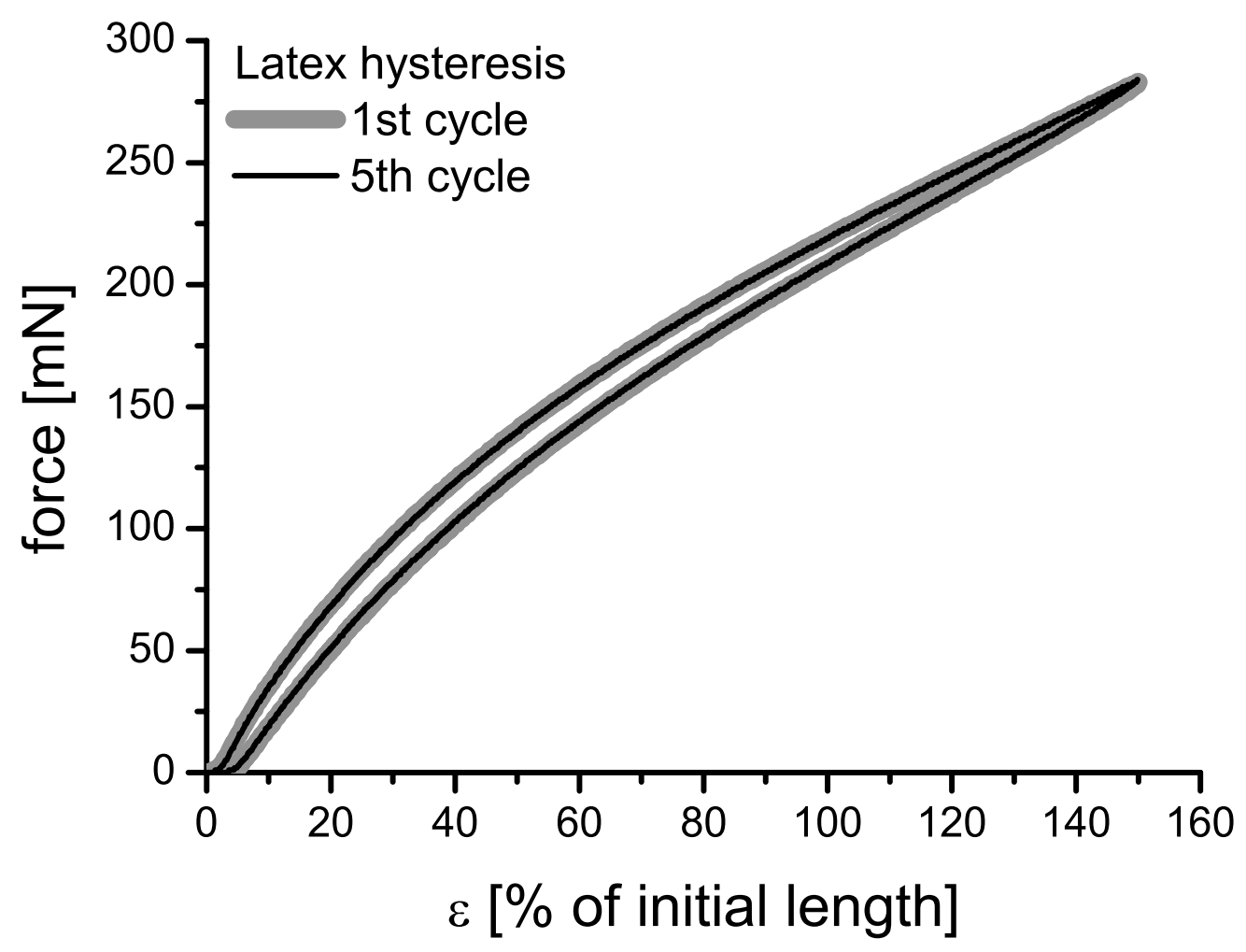

Figure 2.11: First and fifth hysteresis cycle of a latex sample. The different cycles are nearly indistinguishable. The energy loss per cycle is approximately $7 \%$ of the total stretching energy. The sample was approximately $15 \mathrm{~mm} \times 2 \mathrm{~mm} \times 0.125 \mathrm{~mm}$ and is used e.g. for laboratory gloves. Latex exhibits mainly entropic elasticity, the classical model for reversible processes. 


\subsection{Dragline's Mechanical Properties}

MAS combines a tensile strength $\sigma_{\max }$ of $1.1 \mathrm{GPa}$ with an extensibility $\varepsilon_{\max }$ of up to $30 \%$ connected by an elastic modulus $\mathcal{E}$ of $20 \mathrm{GPa}$ for Nephila. The measurement of these properties is outlined in the previous section 2.3. The extensibility exceeds that of nylon (polyamide 6,6) and steel, see figure 2.8. Nylon is also outperformed in tensile strength, see table 2.1. The high extensibility, kept over the fiber's whole extension range, combined with high tensile strength results in a toughness of $160 \mathrm{~J} / \mathrm{m}^{3}$ (total energy $E_{\max }$ per volume until rupture). This value is astonishing for a protein, for comparison nylon exhibits a toughness of $80 \mathrm{~J} / \mathrm{m}^{3}$ and steel $6 \mathrm{~J} / \mathrm{m}^{3}$.

Strongly related to the toughness is the ability to dissipate energy. Especially the spider's major ampullate gland silk is optimized for this purpose because its not only used for the web's frame but also acts as the spider's lifeline. Without internal energy dissipation in the fiber, neither the spider nor her prey would be caught, but instead catapulted back in a spring-like fashion. On the other hand also a specific amount of elastic behavior is useful to reform the web after an impact. $65-68 \%$ of the stretching energy $E$ is dissipated in dragline while only $32-35 \%$ are elastically stored. Although a stretched fiber contracts to its original shape the former extension is 'stored' in the fiber (hysteresis). In a subsequent elongation the stress caused by a special strain is much smaller than before, until the prior maximum extension is crossed. From this point on the stress-strain behavior is the same as for an unstretched fiber, see figure 2.12. This 'elongation history' of dragline can be erased by immersion in water. Wetted the fiber contracts to approximately $50 \%$ of its original length $[6 ; 54 ; 55 ; 56]$. This phenomena is called supercontraction. After drying the fiber is again in the native state as before an extension [57]. A potential structure change which can explain this hysteresis behavior will be presented in chapter 8 . Beside contraction a wetted dragline fiber softens with humidity but without losing significantly in tensile strength and toughness, see chapter 5 .

Table 2.1: Tensile properties of man-made and natural fibers. The toughness describes the energy a fiber can take before rupture per volume. Dragline silk outperforms the other materials in this regard due to its combination of tensile strength and extensibility. The values for Nephila are from measurements performed in the Institute für Röntgenphysik [52; 53], the others are from literature [58; 59].

\begin{tabular}{lcccc}
\hline Material & $\begin{array}{c}\sigma_{\max } \\
{[G P a]}\end{array}$ & $\begin{array}{c}\varepsilon_{\max } \\
{[\% \text { of initial length] }}\end{array}$ & $\begin{array}{c}\mathcal{E} \\
{[\mathrm{GPa}]}\end{array}$ & $\begin{array}{c}\text { Toughness } \\
{\left[\mathrm{MJ} / \mathrm{m}^{3}\right]}\end{array}$ \\
\hline High tensile steel & 1.5 & 0.8 & 200 & 6 \\
Kevlar 47 & 3.6 & 2.7 & 130 & 50 \\
Nylon & 0.95 & 18 & 4 & 80 \\
Bombyx mori & 0.6 & 18 & 6 & 70 \\
Nephila MAS & 1.1 & 30 & 20 & 170 \\
\hline
\end{tabular}




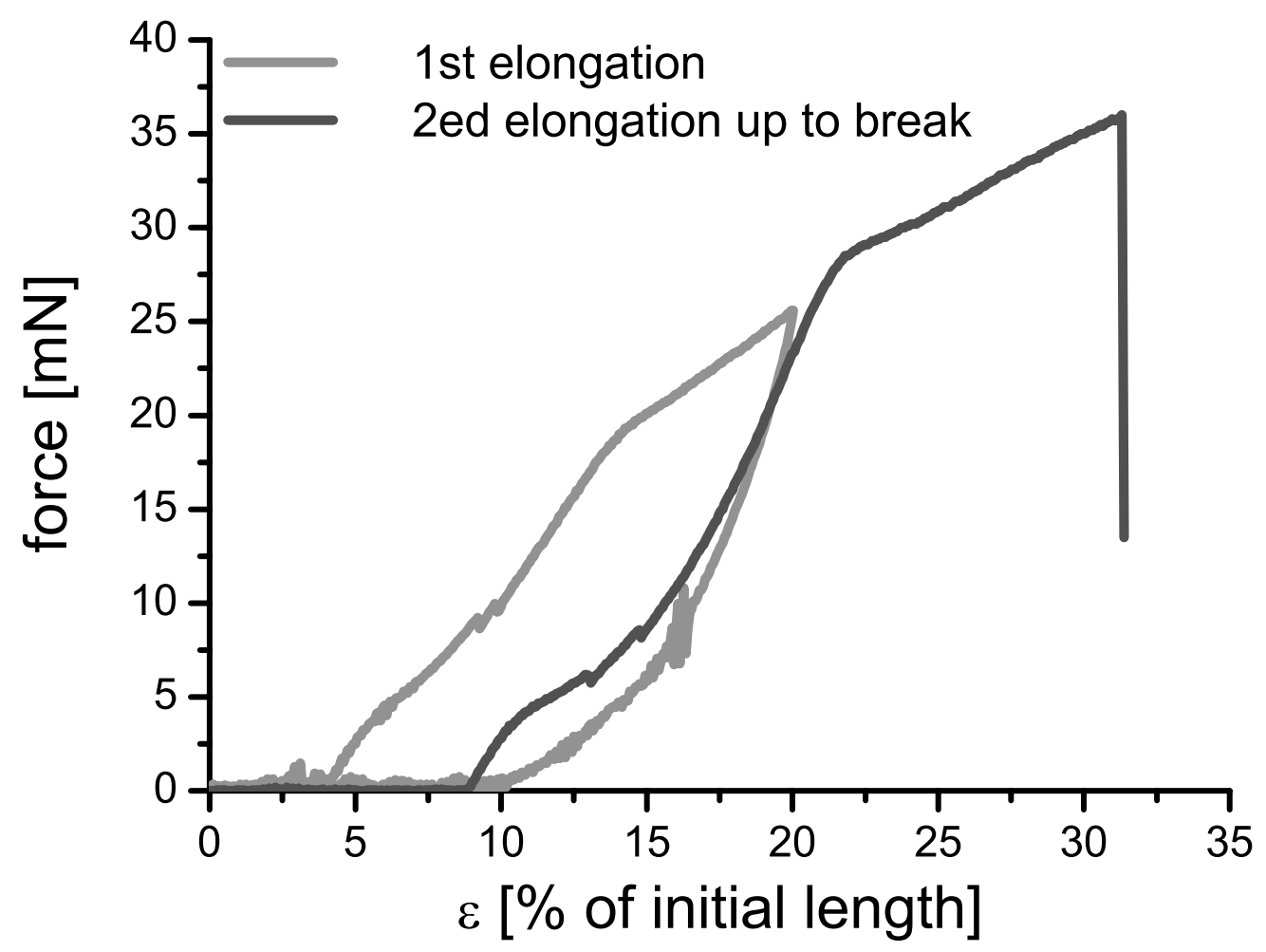

Figure 2.12: Force-strain curve of elongation, relaxation and repeated elongation up to break. The second ascent differs from the first until the first maximum extension is crossed. From there on the force-strain curve is equal to the force-strain curve obtained without relaxation. Data by $\mathrm{T}$. Vehoff [52; 53]. 


\subsection{Models}

Over the years some models have been developed to describe the stress-strain behavior of spider silk but until now none of them has been able to describe all modes of spider silk's properties. Some tried to expand models developed for other polymers, like polyethylen, to spider silk by adding hydrogen bonds to the otherwise assumed ideal polymer chains. Others described the dragline's response to mechanical load by strain induced unfolding of proteins [60;61] or tried a phenomenological approach [39; 62]. The two most successful models will be presented here shortly.

\subsubsection{Hierarchical Chain Model}

The hierarchical chain model was originally developed by Haijun Zhou and coworkers for spider capture silk which shows a behavior similar to wet dragline but exceeds it in extensibility [63; 64]. Two observation were the starting point of this model:

1. High extensibility, interpreted as a high amount of extra length, stored in the relaxed fiber.

2. Exponential force-strain curve (figure 2.14), which gives rise to the assumption that some fraction of extra length can be pulled out easily, while other fractions are harder to be pulled out.

In the model both observations are combined with the hierarchical organization of proteins (primary to quaternary structure), the polymer is composed of a number of different structural motifs, which are organized in hierarchy levels. Each of these hierarchy levels $h$ consist of a number $m_{h}$ of identical structural units $M_{h+1}$ which again are composed of $m_{h+1}$ identical subunits $M_{h+2}$, see figure 2.13. The highest level is the fiber itself, denoted as $h=0$, structural motifs of lower levels are for example $\beta$-sheets or helices.

An external force $f$ causes an extension $x_{0}(f)$ which consists of the positional rearrangement of the the subunits $\Delta x_{0}(f)$ plus the extension $x_{1}(f)$ of the $m_{0}$ subunits $M_{1}$.

$$
x_{0}(f)=\Delta x_{0}(f)+m_{0} x_{1}(f)
$$

The same holds true for the extension of all following subunits $h$.

$$
x_{h}(f)=\Delta x_{h}(f)+m_{h} x_{h+1}(f)
$$

This continues through all hierarchy levels and therefore the extension of the whole fiber can be written as:

$$
x(f)=\Delta x_{0}(f)+\sum_{h=0} m_{0} m_{1} \ldots m_{h} \Delta x_{h+1}(f)
$$


This can be calculated for any force field, but for the sake of simplicity the response of every hierarchy level $h$ is assumed to be linear and proportional to the unit's relaxed contour length $L_{h}$ :

$$
\Delta x_{h}(f)= \begin{cases}\alpha L_{h} f / f_{h} & f<f_{h} \\ \alpha L_{h} & f \geq f_{h}\end{cases}
$$

where $\alpha$ is a dimensionless proportional constant and $f_{h}$ a characteristic force to displace and rearrange the submodules. After $f_{h}$ is reached, no more extra length can be gained. For the rearrangement of the subunits sacrificial bonds between the subunits have to be broken. They are assumed to be stronger for deeper hierarchy levels $h$ and therefore is the characteristic force $f_{h}$ :

$$
f_{h+1}=\beta f_{h} \quad \text { with } \beta=1 .
$$

This ensures that the extra length hidden in the subunits reveals from highest to lowest hierarchy level. Together with the equation 2.3 and 2.4, the condition $f_{h-1}<f \leq f_{h}$ leads to the following differential equation:

$$
\frac{d x(f)}{d f}=\sum_{h^{\prime}-h} \frac{\alpha L_{0}}{f_{h}^{\prime}}=\frac{\alpha \beta L_{0}}{\beta-1} f^{-1}
$$

Which can be solved by an exponential force-strain relationship:

$$
f \approx \exp (x / l) \quad \text { with } \quad l=\frac{\alpha \beta}{\beta-1} L_{0}
$$

This model agrees very well with experimental data found for spider silk, see figure 2.14. By comparison with experimental data it was found that $\beta \approx 2, f_{0} \approx$ $10^{-4} N, \alpha=0.3$ and $4-5$ levels of hierarchy are suitable for spider capture silk. 


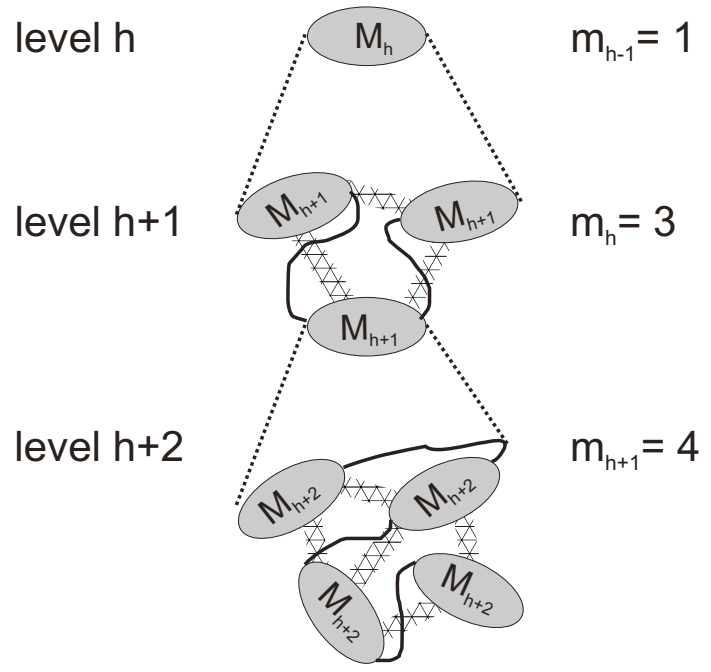

$X X X X X Y$ scarificial bonds

Figure 2.13: Schematic of the hierarchical chain model, according to $\mathrm{H}$. Zhou et al. [63]. At each hierarchy level $h$ a structural module $M_{h}$ is composed of $m_{h}$ identical units $M_{h+1}$ which themselves are composed of $m_{h+1}$ subunits $M_{h+2}$.

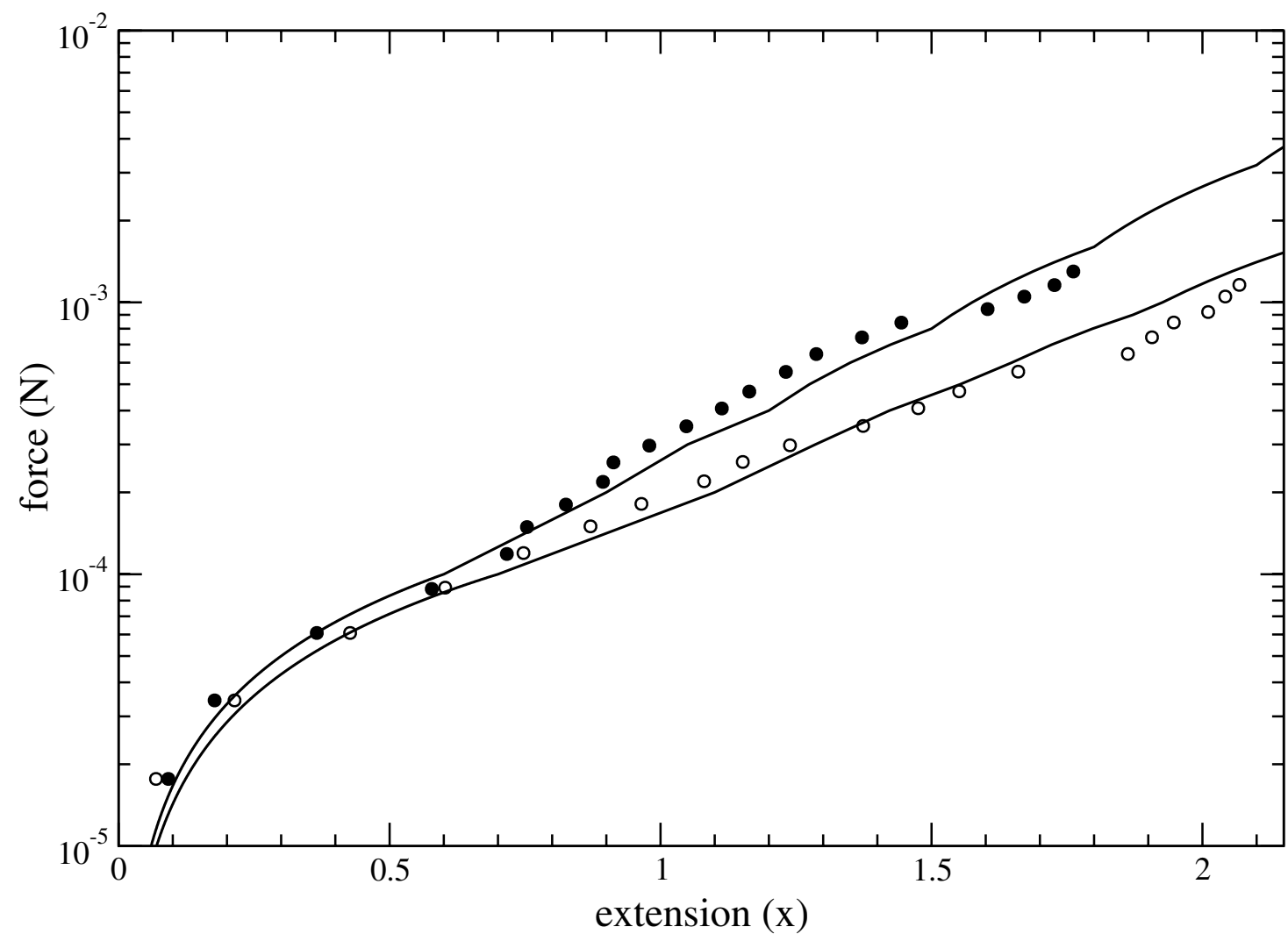

Figure 2.14: Comparison between experiment (circles / dots) and simulation (lines), extension( $\mathrm{x}$ ) $=\left(l-l_{0}\right) /\left(l_{0}\right)$. The simulated curves follow $f \approx \exp (x / l)$ and agree very well with experimental data, from [63]. 


\subsubsection{Molecular Modeling of Spider Silk Elasticity}

The model by Yves Termonia is among the models for dragline silk the most successful. The advantage of the used molecular dynamics simulation is the ability to deal with a higher complexity than could be dealt with analytically.

Starting point of the simulation is a simplified, two-dimensional network of flexible amorphous chains reinforced by stiff crystallites [65; 66; 67], see figure 2.15. Chain entanglements and hydrogen bonds between the chains are allowed [68; 69]. The elastic modulus of a hydrogen bond $\mathcal{E}_{h}$ is assumed to be $\mathcal{E}_{h}=4 \mathrm{GPa}$. The amorphous chains are assumed to be freely jointed and entropic as in polyethylene, accordingly the elastic tensile modulus of $\mathcal{E}_{a} \approx 7 \mathrm{MPa}$ at room temperature and the distance between two entanglements of $3.7 \mathrm{~nm}$ for polyethylene are used in the model.

For the sake of simplicity and to reduce the calculation time the entanglements are assumed to be arranged on a regular lattice. This simplification also leads to a replacement of the hydrogen bonds between the chains by "global" hydrogen bonds interconnecting adjacent entanglements.

The $\beta$-sheet crystallites $(20 \AA \times 60 \AA)$ are viewed as stiff $\left(\mathcal{E}_{c}=160 \mathrm{GPa}\right)$, multifunctional cross links, connecting different amorphous strands. Further the amorphous chains connected in crystallite are constraint, forming a layer of stiffer strands around the crystallite. The layers are supposed to be six times stiffer than the undisturbed amorphous bulk which means $\mathcal{E}_{\text {layer }}=6 \mathcal{E}_{a}$.

The network's response to an applied elongation $\varepsilon$ is simulated in a series of small strain increments $\delta \varepsilon$ with local stress and strain values given by

$$
\sigma_{i}=\mathcal{E}_{i} \varepsilon_{i}
$$

For an amorphous chain strand $i$ the relation

$$
\sigma=\mathcal{E}_{a} n_{e}^{1 / 2} \frac{1}{3} \mathcal{L}\left[\lambda_{i} / n_{e}^{1 / 2}\right]-\sigma_{0}
$$

was used, where $\lambda_{i}=\left(1+\varepsilon_{i}\right)$ denotes the local draw ration, $n_{e}$ the number of segments per chain and $\mathcal{L}$ the Langevin function:

$$
\mathcal{L}(x)=\operatorname{coth}(x)-\frac{1}{x}
$$

Only displacements in the direction of the fiber axis are considered. Lateral displacements are supposed to be only due to area conservation (volume conservation in 3D). Further the amorphous chains break when their draw ratio exceeded their maximum $\lambda_{\max }=n_{e}^{1 / 2}$. The hydrogen bonds break and deform at a rate

$$
v_{i}=\tau \exp \left[-\left(U-\beta \sigma_{i}\right) / k_{b} T\right]
$$

where $\sigma_{i}$ is the local stress, $\tau$ the thermal vibration frequency, $U$ activation energy and $\beta$ the activation volume. The values of $U=35 \mathrm{kcal} / \mathrm{mol}$ and $\beta=4.74 \AA$ are chosen due to experimental findings.

With these settings the curves displayed in figure 2.16 and 2.17 were obtained 
which resemble the experimental findings quite well. The initial linear curve shape then can be attributed to the hydrogen bonds, see figures 2.16. Breaking of $\mathrm{H}$-bonds leads to the yield point. Beside the reinforcement by $\beta$-sheet crystallites the constrainment of the chains contributes significantly to the fibers tensile properties, see figures 2.16. Without the stiffer layer the mechanical behavior is dominated by the amorphous chains, the systems response to an applied force is softer than with the layer. The softening by rising humidity could be reproduced by a reduction of hydrogen bonds in the amorphous matrix, see figure 2.17. All this makes this model the most useful to study the mechanical properties, although it does not cover hysteresis behavior.

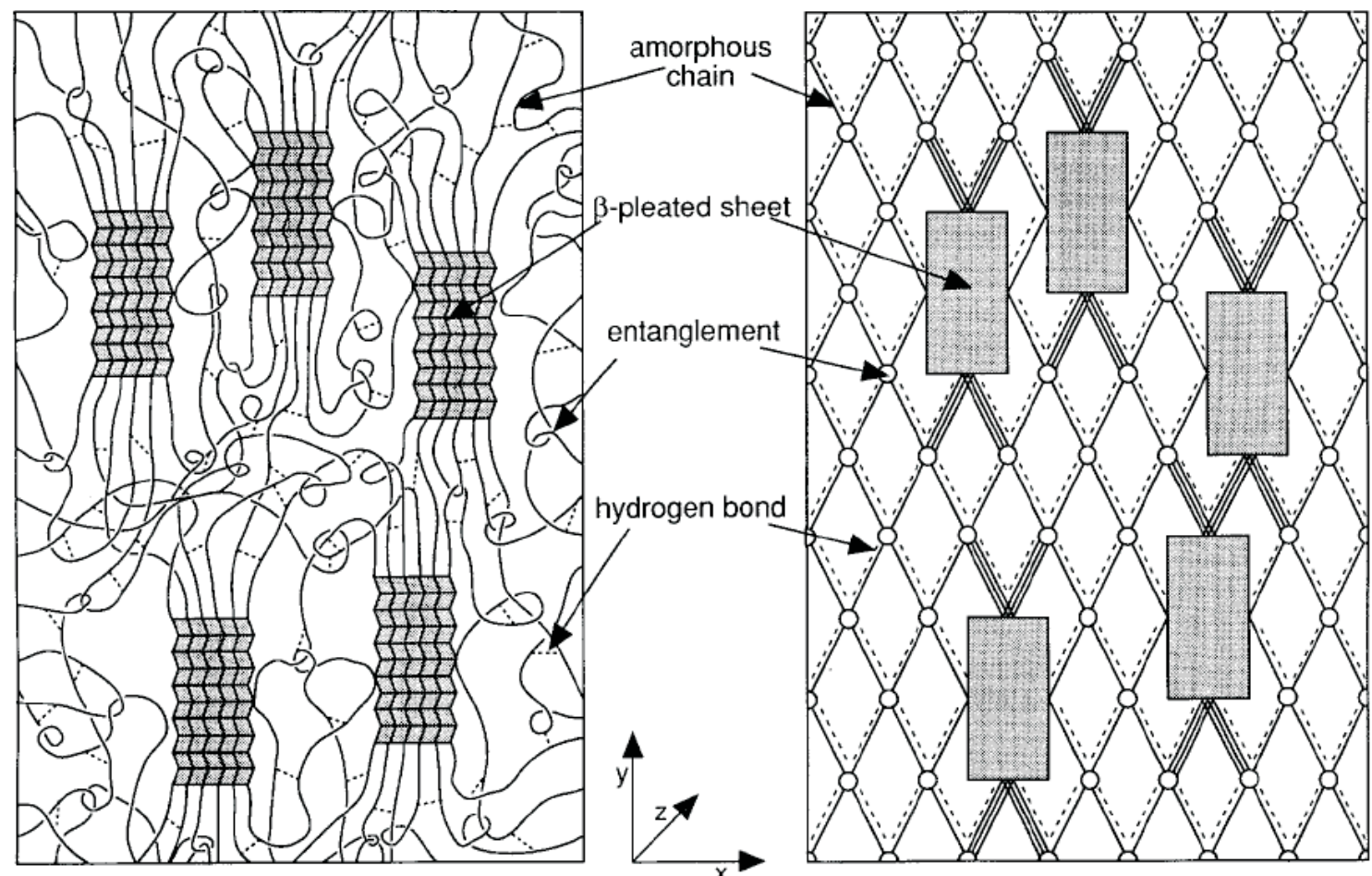

$6 \mathrm{~nm}$

Figure 2.15: Left Schematic of the nanoscale structure of spider silk. Right Simplified dragline silk inspired network used by Y. Termonia for molecular modeling of spider silk elasticity. The amorphous matrix is replaced by a regular latter of entanglements (circles), hydrogen bonds between chains by bonds between entanglements (dotted lines). From publication [65] 


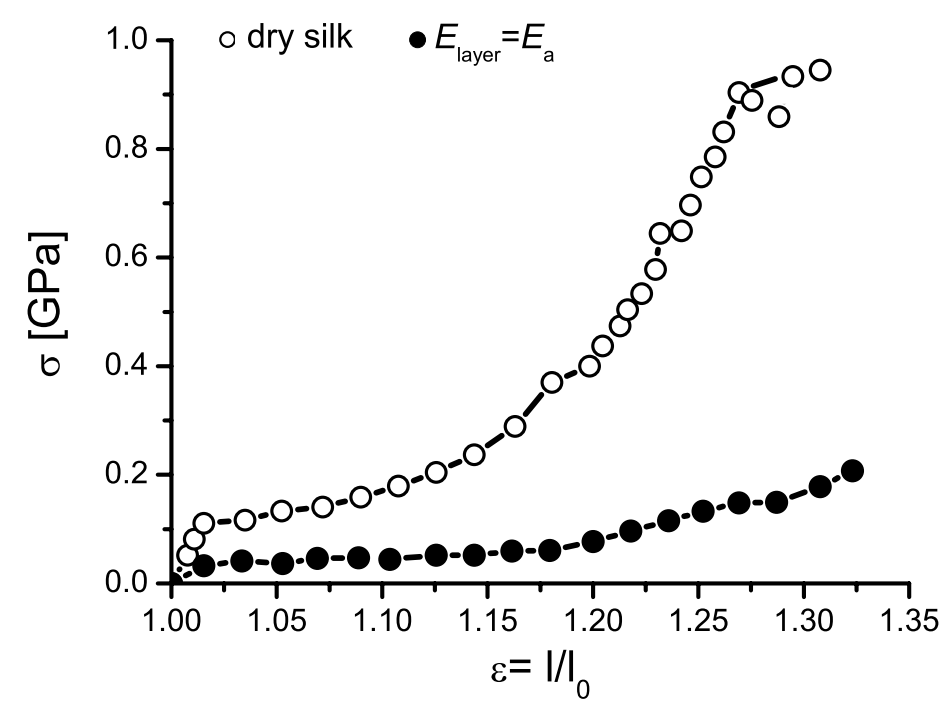

Figure 2.16: Simulated stress-strain curve for dry spider silk and with $\mathcal{E}_{\text {layer }}=\mathcal{E}_{a}$. Graph according to Y. Termonia [65].

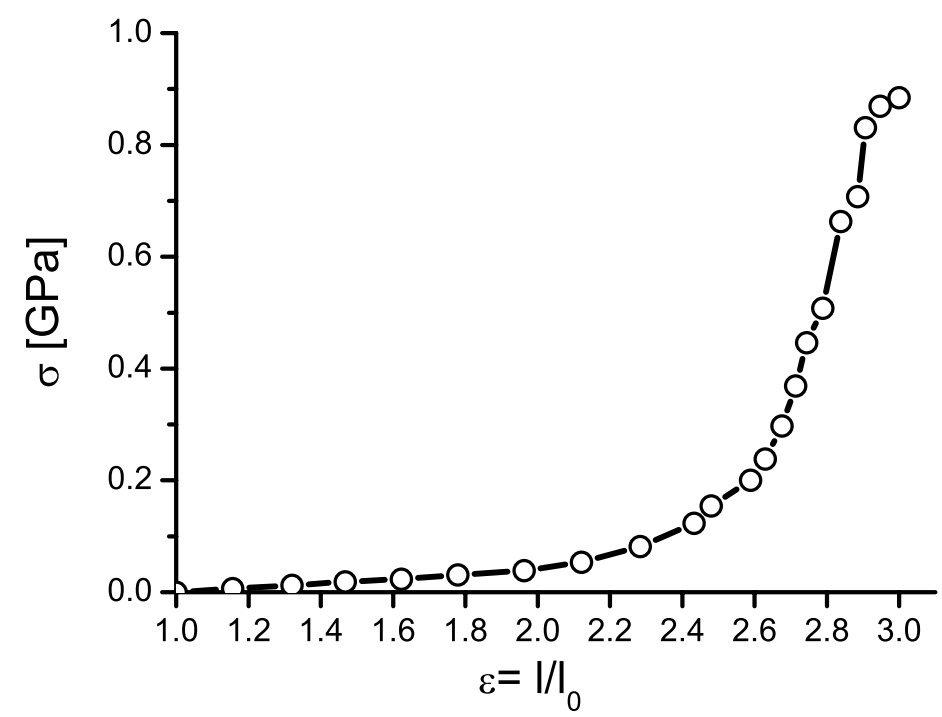

Figure 2.17: Simulated stress-strain curve for unrestrained wet spider silk. "Wet" means that the hydrogen bonds in the amorphous matrix are substituded by water. Graph according to Y. Termonia [65]. 



\section{Chapter 3}

\section{X-Ray Diffraction, Instrumentation and Data Analysis}

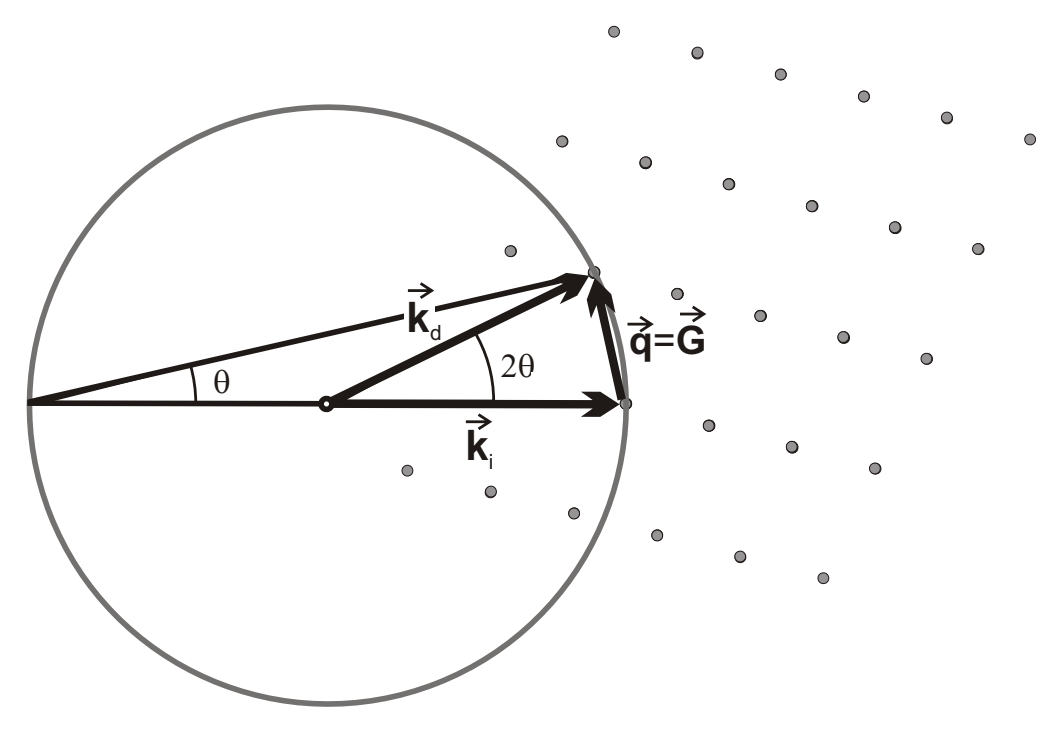

Figure 3.1: Two-dimensional representation of the Ewald-Sphere. Whenever the scattering vector $\vec{q}$ (difference between incident $\vec{k}_{i}$ and diffracted $\vec{k}_{d}$ wave) equals a vector $\vec{G}$, connecting two points of the reciprocal crystal lattice, a reflection occurs.

With the first experiments of Max von Laue, Walther Friedrich and Paul Knipping on copper sulfate, in 1912, X-ray diffraction has become the standard method for structure determination [70]. The experiment did not only prove that X-rays are electromagnetic radiations similar to light but also that crystals have a regular, periodic structure.

The smallest repeating unit with the same symmetry as the whole crystal is called unit cell [71]. A crystal can be composed by a subsequent arrangement of such identical unit cells. The position $\vec{R}_{n}+\vec{r}_{j}$ of every atom in a crystal can then be given by superposition of the lattice vector $\vec{R}_{n}$ denoting the unit cell the atom 
lays in and its position $\vec{r}_{j}$ within the unit cell. The X-ray scattering function $\mathcal{S} \mathcal{F}(\vec{q})$ factorizes into the product of lattice sum $S$ and structure factor $F$ :

$$
\begin{aligned}
\mathcal{S F}(\vec{q}) & =S(\vec{q}) \cdot F(\vec{q}) \\
S(\vec{q}) & =\sum_{\vec{R}_{n}} e^{i \vec{q} \cdot \vec{R}_{n}} \\
F(\vec{q}) & =\sum_{\vec{r}_{j}} f_{i}(\vec{q}) e^{i \vec{q} \cdot \vec{r}_{j}}
\end{aligned}
$$

The lattice sum $S(\vec{q})$ describes the diffraction from the unit cells arrangement. The unit cell's basis vectors are $\vec{a}, \vec{b}$ and $\vec{c}$ and therefore the lattice vectors are of the form

$$
\vec{R}_{n}=n_{a} \vec{a}+n_{b} \vec{b}+n_{c} \vec{c}
$$

where $n_{a}, n_{b}$ and $n_{c}$ are integers. The form factor $F(\vec{q})$ describes the diffraction from the atoms in the unit cell, with $f_{i}$ the atomic scattering factor. The scattering function $\mathcal{S F}(\vec{q})$ vanishes due to destructive interfernce unless the scattering vector $\vec{q}$ fulfills:

$$
\vec{q} \cdot \vec{R}_{n}=2 \pi \times \text { integer }
$$

This is the case when the scattering vector $\vec{q}$ equals a vector $\vec{G}$ of the reciprocal lattice defined by

$$
\begin{aligned}
\vec{G} & =h a^{*}+k b^{*}+l c^{*} \\
a^{*} & =2 \pi \frac{b \times c}{a \cdot(b \times c)} \\
b^{*} & =2 \pi \frac{c \times a}{a \cdot(b \times c)} \\
c^{*} & =2 \pi \frac{a \times b}{a \cdot(b \times c)}
\end{aligned}
$$

where $h, k$ and $l$ are all integers. The product of such lattice vectors from recipro$\operatorname{cal}(\vec{G})$ and direct space $\left(\vec{R}_{n}\right)$ is

$$
\vec{G} \cdot \vec{R}_{n}=2 \pi\left(h n_{a}+k n_{b}+l n_{c}\right)=2 \pi \times \text { integer }
$$

and always fulfills the requirement stated in equation 3.5. When ever the LaueCondition

$$
\vec{q}=\vec{G}
$$

is satisfied the interference is constructive, $\mathcal{S F}$ becomes of the order of the number of unit cells $N=N_{a} \cdot N_{b} \cdot N_{c}$, a reflection occurs. The Laue-Condition is illustrated by the Ewald-Sphere shown in figure 3.1. 
With $u_{j}, v_{j}, w_{j}$ indicating the positions of all atom's $M$ within the unit cell, the intensity $I$ of a certain reflection $h, k, l$ becomes:

$$
\begin{aligned}
I(h, k, l) & =|S(h, k, l)|^{2} \cdot|F(h, k, l)|^{2} \\
S(h, k, l) & =\frac{\sin \left(\pi N_{a} h\right)}{\sin (\pi h)} \frac{\sin \left(\pi N_{b} k\right)}{\sin (\pi k)} \frac{\sin \left(\pi N_{c} l\right)}{\sin (\pi l)} \\
F(h, k, l) & =\sum_{j=1}^{M} f_{i} e^{-i 2 \pi\left(u_{j} h+v_{j} k+w_{j} l\right)}
\end{aligned}
$$

An analogous description of X-ray scattering was introduced by $W$. H. Bragg and W. L. Bragg in 1913 [72]. It proposes that in a crystal the atoms are arranged in virtual planes. The scattering of X-rays at such an ensemble of parallel planes is constructive whenever the retardation between two rays scattered at neighboring planes equals a multiple of an integer of the wavelength:

$$
2 d \sin \theta=n \lambda \quad \text { Bragg condition }
$$

Where $n$ and $m$ are natural numbers and $d$ is the distance between the planes. The notation $h, k, l$ also denotes the planes in a three-dimensional crystal from which a reflection originates and therefore interconnects the two ways of describing $X-$ ray diffraction introduced by Laue and Bragg.

Any plane in a crystal cuts the crystal's main axes in one, two or three points. The axes intercepts where the axes $x, y, z$ are cut by the plane may be $1 / 3,-1,2$ and the inverse of these intercepts $3,-1,1 / 2$. The set of minimal integers with the same ratio is $6,-2,1$, see figure 3.2. These are the Miller indices. For the general case they are denoted as $h, k, l$. All planes with the same Miller indices are parallel and contribute to a reflection. Both planes and reflections are described by the same Miller indices. To distinguish them reflections are written with parentheses $(h \mathrm{kl})$ and ensembles of Bragg planes are written in square brackets [ $h \mathrm{kl}]$, furthermore negative Miller indices are referred to with a top line, so $(6-21)$ becomes (62)1). The distance $d$ between the Bragg planes can be directly retrieved from the experiment by the angle $2 \theta$ between incident and diffracted beam, as stated in equation 3.15 and shown in figure 3.1. If additionally the unit cell's symmetry and the Miller indices are known the lattice constants $a, b, c$ can be calculated. E.g. for an orthorhombic unit cell, as in anti-parallel $\beta$-sheets, the relation is:

$$
q=\frac{2 \pi}{d}=2 \pi \sqrt{\frac{h^{2}}{a^{2}}+\frac{k^{2}}{b^{2}}+\frac{l^{2}}{c^{2}}}
$$

But not only the crystal's lattice constants can be retrieved but also the crystal's length $L$. Normally a X-ray beam is much bigger than the illuminated crystal, so a peak's full width at half maximum (fwhm) depends on the total number of Bragg planes in the crystal. Thus the crystal's length $L$ perpendicular to the planes can be calculated. This is done by the Debye-Scherrer formula:

$$
L=\frac{k \lambda}{\beta \cos \theta}=\frac{k \lambda}{\Delta \theta \cos \theta} \text { Debye-Scherrer formula }
$$


Where $\beta$ is the fwhm in radiant and $k$ is a factor which takes the crystal's shape into account. For the analysis of our experiments $k=0.9$ was used which lays between $k=0.89$ for a spherical crystal and $k=0.94$ for a cube.

In X-ray physics it is common to use the scattering vector $q$ instead of $\theta$ because $\theta$ always requires to give also the wavelength. The scattering vector $q$ and $\theta$ are related by the Bragg condition as follows:

$$
\begin{aligned}
q & =\frac{2 \pi}{d} \\
& =\frac{4 \pi \sin \theta}{\lambda}
\end{aligned}
$$

Commonly $q$ is given in reciprocal Ångströms which corresponds to $10^{-10}$ meters. Thus the Debye-Scherrer formula becomes:

$$
\begin{aligned}
L & =\frac{k 4 \pi \lambda}{4 \pi \Delta \theta \cos \theta} \\
\Rightarrow L & =\frac{k \cdot 4 \pi}{d q}
\end{aligned}
$$

With the auxiliary calculations:

$$
\begin{aligned}
2 d \sin \theta & =\lambda \quad \text { Bragg condition } \\
\Rightarrow q & =\frac{4 \pi}{\lambda} \sin \theta \\
\frac{d q}{d \theta} & =\frac{4 \pi}{\lambda} \cos \theta
\end{aligned}
$$

Actually the Debye-Scherrer formula was derived for large crystals where the number of Bragg planes $m$ is significantly larger than their distance $d$. This is not the case for dragline silk. Normally there are around five Bragg planes along the $\beta$-sheets $x$ - and $y$-axis. A numerical analysis of the deviation from the theoretical crystal size $L_{\text {theo }}$ and the crystallite size $L_{D S}$ calculated by Debye-Scherrer formula can be found for example in [53]. For five Bragg planes the deviation is not bigger than 1.8 percent. An overview of the deviations for small numbers of Bragg planes is given in table 3.1. Down to three Bragg planes the deviation is not bigger than $6 \%$ which is well within the experiments' sensitivity, see chapter 7 to 9 .

Table 3.1: Deviation $\sigma$ of the crystallite sizes $L$ calculated via Debye-Scherrer formula from the real crystallite size against the number of Bragg planes $m$. The deviation is given in percent. From [53].

\begin{tabular}{c|c|c|c|c|c|c|c|}
$m$ & 2 & 3 & 4 & 5 & 6 & 7 & 8 \\
\hline$\sigma[\%]$ & 12.9 & 5.16 & 2.81 & 1.77 & 1.22 & 0.892 & 0.681
\end{tabular}




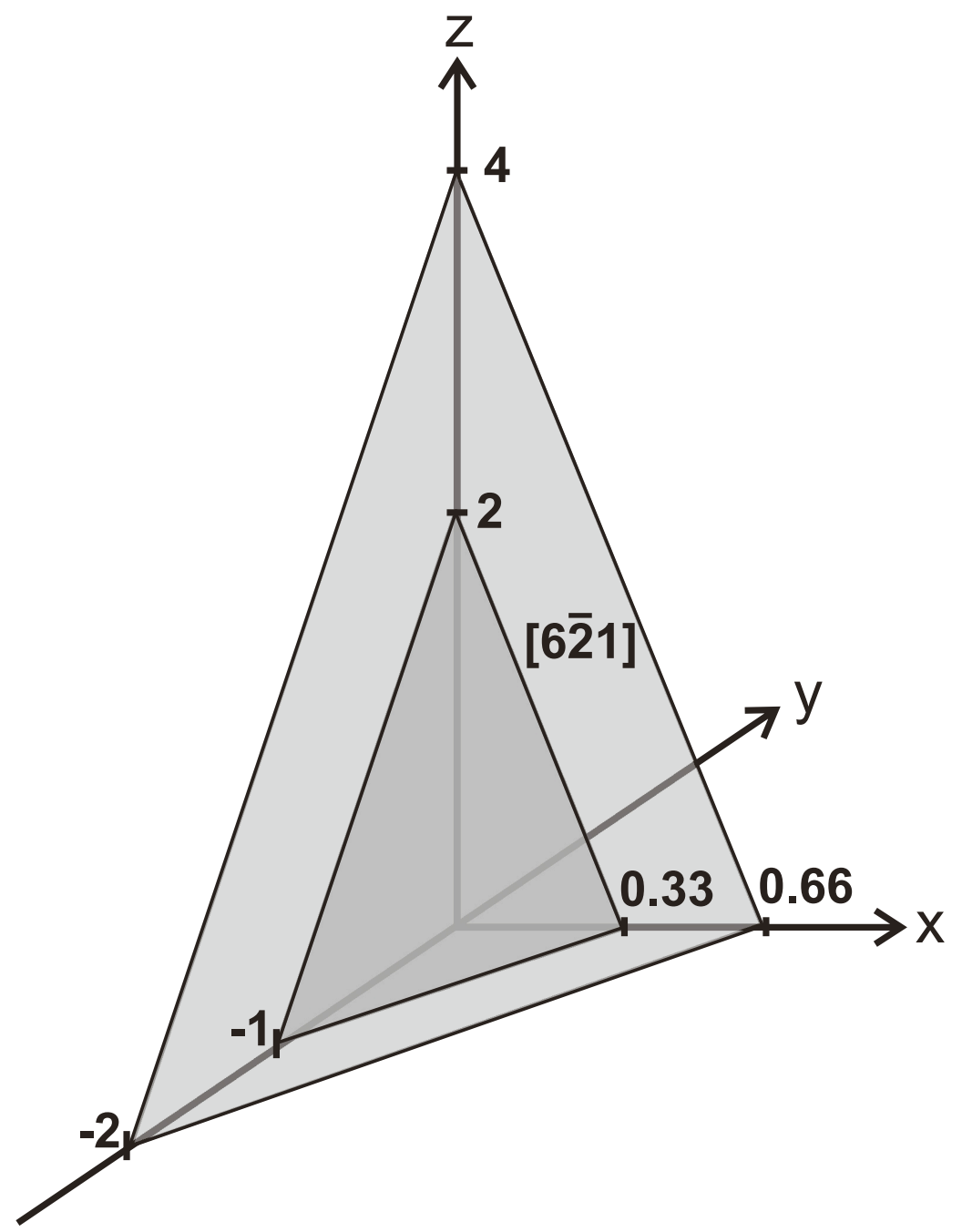

Figure 3.2: Two [62̄1] planes. The axes intercepts where the planes $c$ ut the axes are $1 / 3,-1,2$ and $2 / 3,-2,4$, respectively. The inverse of these intercepts are $3,-1,1 / 2$ and $3 / 2,-1 / 2,1 / 4$. The set of minimal integers with the same ratio is $6,-2,1$. All planes with the same Miller index are parallel and contribute to the same reflection, in this case (62)1).

\subsection{Fiber Diffraction}

A powder consists of a high number of randomly distributed crystallites so that for every possible reflection at least one crystallite has the appropriate orientation. The formerly distinct reflections then become rings around the incident beam (Debye-Scherrer cones). Diffraction from a fiber can be considered as a limit of scattering from an anisotropic powder in which the same crystal axis, here denoted as $z$-axis, of all crystals in the sample is aligned along the fiber axis, while the orientation of $x$ - and $y$-axis is isotropic [73]. Due to this geometry it is useful to split the scattering vector $\vec{q}$ into his components parallel $q_{\|}$and perpendicular $q_{\perp}$ to the fiber axis, whereas $|q|=\sqrt{q_{\|}^{2}+q_{\perp}^{2}}$. Correspondingly there are two principal scattering planes, see figure 3.3:

meridionial plane the scattering vector $\vec{q}$ has only a component parallel to the 


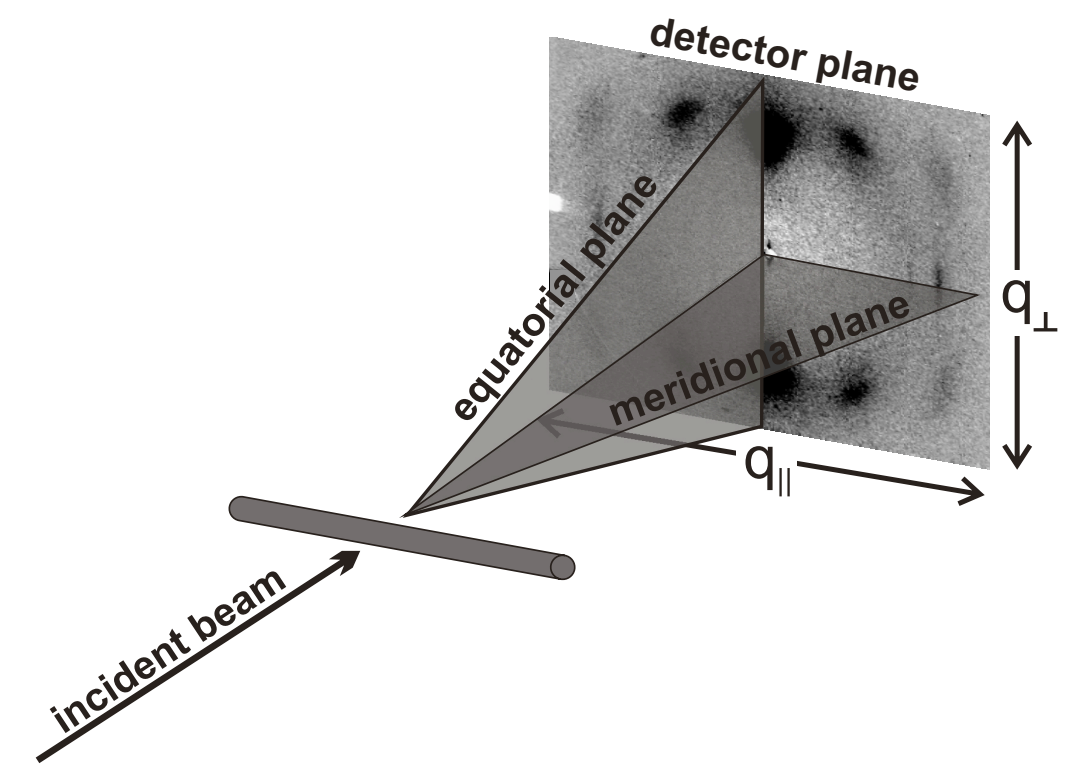

Figure 3.3: Schematic of fiber diffraction. The sample geometry suggests to spilt the scattering vector $\vec{q}$ in components parallel $q_{\|}$and perpendicular $q_{\perp}$ accordingly a meridional and equatorial plane are defined.

fiber axis $\vec{q}=\left(q_{\|}, 0\right)$.

equatorial plane the scattering vector $\vec{q}$ has only a component perpendicular to the fiber axis $\vec{q}=\left(0, q_{\perp}\right)$.

If additional orientations of the crystallites exist in the fiber other phenomena occur. For example the crystallites in kevlar, which also has two main equatorial relfections, are arranged radial in a star like pattern. This arrangement causes the intensity of the equatorial reflections to depend strongly on the beam's position on the fiber [74; 75]. Only when the beam is centered on the fiber both reflections have the same intensity.

In spider silk the only preference of the crystallites is the predominate alignment of the $z$-axis along the fiber axis. The main challenge in X-ray scattering on spider silk is to overcome the weak scattering strength of spider silk [9]. One way to do so is to increase the scattering volume in the beam by winding up a high number of silk fibers. If the winding is done carefully, none of the information about the crystallites' distribution within the fiber is lost, see figure 3.4. Another possibility is to use a more brilliant source like an undulator. Such sources enable even single fiber measurements which will be discussed in chapter 8 and 9 . 


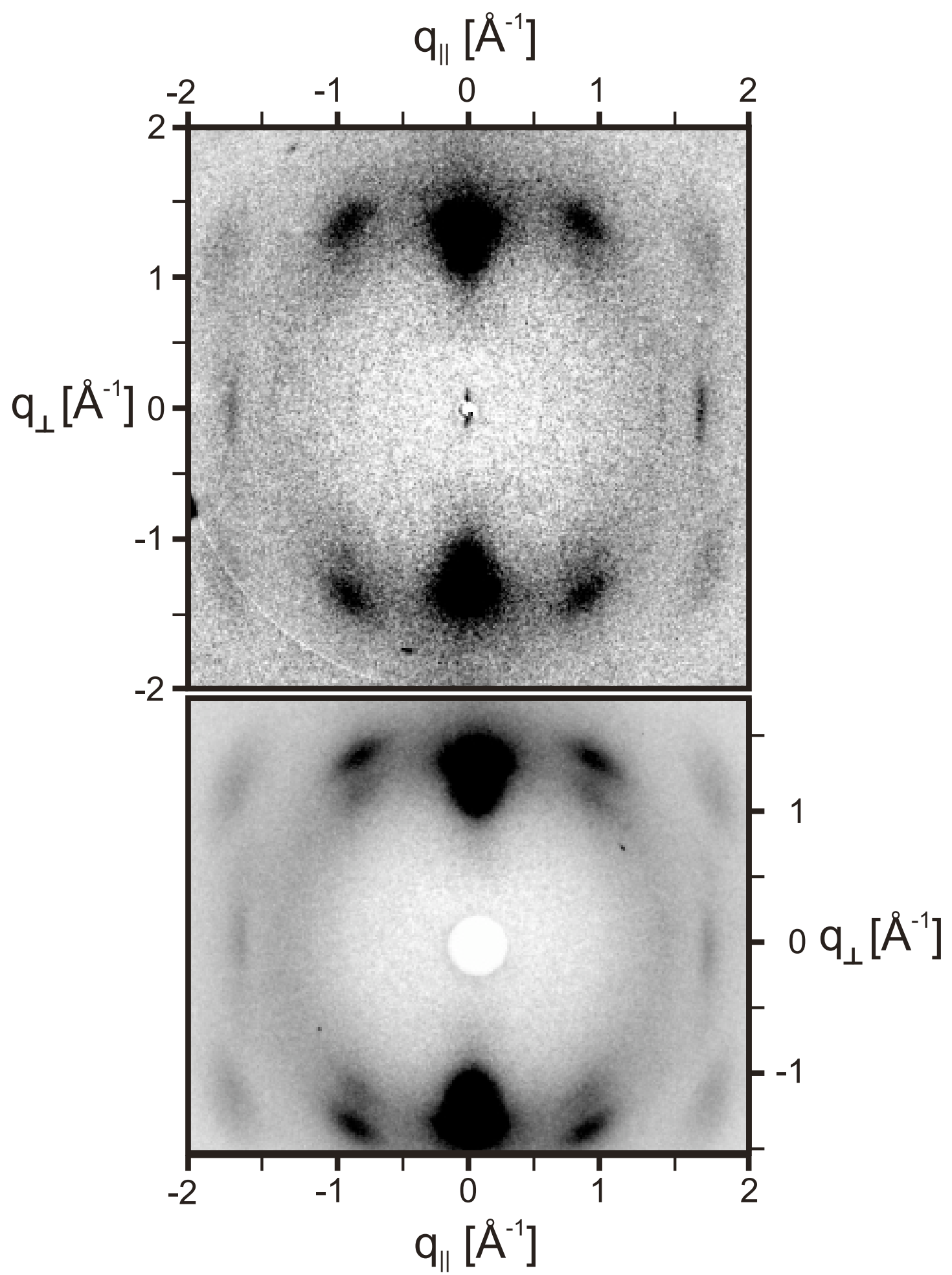

Figure 3.4: Top: Diffraction from a single NM dragline fiber. The data have been taken at the ESRF's microfocus beam line ID 13 at ambient conditions, wavelength $\lambda=0.97 \AA(12.7 \mathrm{keV})$, illumination time 11sec. The indexing is given in figure 3.9. Bottom: Diffraction from a fiber bundle of approximately 400 NC dragline threads, measured at HASYLAB beamline D4 at ambient conditions. Both patterns cover a comparable q-range, wavelength $\lambda=0.97 \AA(10.9 \mathrm{keV})$, illumination time 10min. 


\subsection{D-Patterns and Fiber Bundles}

Diffractometers operating with a X-ray tube source are available in most X-ray laboratories but do not provide a sufficient flux to obtain diffraction patterns from single spider silk fibers. In order to study the structure of spider silk with such instruments it is necessary to increase the sample's scattering volume. For this purpose fiber bundles with 400-900 fibers were produced. These bundles were approximately $4 \mathrm{~mm}$ long and $2 \mathrm{~mm}$ high. By this sample configuration it was possible to study the fibers' structure with the institute's diffractometer WENDI without losing the orientational information.

\subsubsection{WENDI}

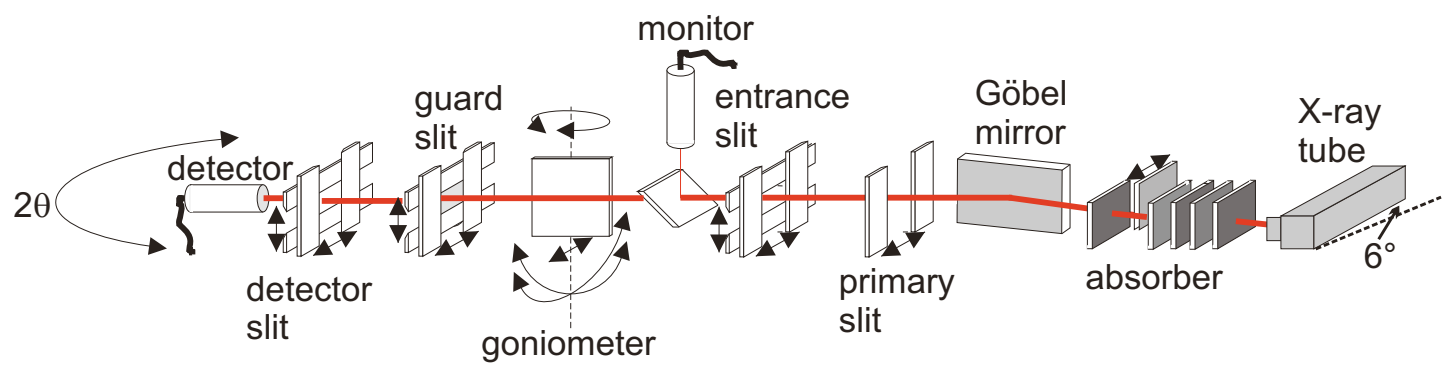

Figure 3.5: Schematic of the WENDI diffractometer in the Institute für Röntgenphysik [76].

The institute's diffractometer WENDI is a z-axis diffractometer operating with a line focus $(0.4 \mathrm{~mm} \times 12 \mathrm{~mm})$. For the analysis of spider silk it was operated in transmission configuration. The instrument is equipped with a sealed tube source, bent collimating Göbel multilayer mirrors, motorized slits, and a fast $\mathrm{NaI}$ scintillation counter (Cyberstar, Oxford Instruments, Abingdon, UK) controlled by the scientific software SPEC (Certified Scientific Software, Cambridge, USA). The multilayer mirrors and an additional Ni-foil were optimized to select $\mathrm{Cu}-\mathrm{K} \alpha$ radiation ( $\mathrm{E}=8.0 \mathrm{keV}, \lambda=1.54 \AA$ ). The (120)- and (200)-reflections were chosen to be observed. Therefore the fiber bundle was mounted vertically so that the horizontal scattering plane corresponded to the sample's equatorial plane, see figure 3.3. The instrument's resolution strongly depends on the beam size, theoretically a resolution $\Delta \theta=0.001 \mathrm{deg}$ is possible. For the experiments described in chapter 7 a step size of $0.1 \mathrm{deg}$ and an illumination time of $60-90 \mathrm{sec}$ per step were the best compromise of resolution and experiment time. A calibration to allocate the reciprocal space to the scattering signal, as for the experiments performed at the ESRF, see section 3.3.3, is unnecessary after the position of the primary beam is set as zero. Correspondingly, the analysis was performed in $2 \theta$-notation and followed the methods described in section 3.4. The experimental results are given in chapter 7. 


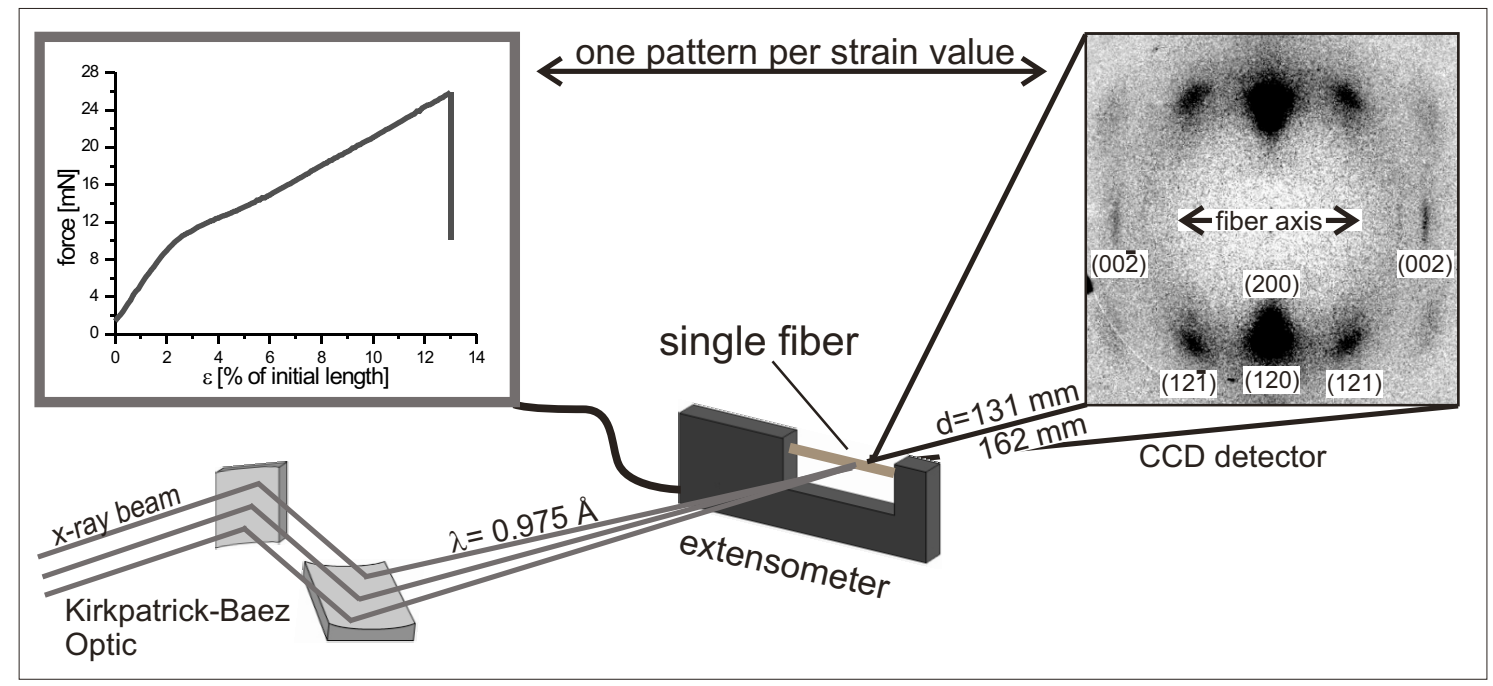

Figure 3.6: Schematic of the experimental setup at ID13. The monochromatic beam (Si-111 monochromators not shown) from the undulator is focused by a pair of crossed Kirk-Patrick Beaz mirros. At the sample the beam has a size of approximately $7 \mu \mathrm{m}$. The single fiber is mounted in a LEX 810 extensometer which records the force-strain curves, simultaneously the diffraction pattern is recorded by a MAR 165 CCD, positioned 131-162 mm behind the sample. The image in the upper left corner shows a diffraction pattern with Miller indices.

\subsection{D-Patterns - Single Fiber Diffraction}

The single fiber experiments were performed at the Microfocus Beamline ID13 at European Sychrotron Radiation Facility ESRF in 2005 and 2006. With the highly brilliant X-ray beam available it is possible to obtain diffraction patterns from single dragline fibers $[8 ; 9 ; 10]$.

\subsubsection{Microfocus Beamline ID13 equipped with KB-Optics}

The principal aim of the Microfocus Beamline ID13 is to provide small focal spots for wide-angle $X$-ray diffraction (WAXS) and small-angle $X$-ray scattering (SAXS). All ambient experiments were performed at the beamline's standard ambient conditions around $50 \%$ relative humidity and $23^{\circ} \mathrm{C}$ and with the beamline's setup described here. The beamline's incident photon energy is variable between about $5 \mathrm{keV}$ (Be cut-off) and about $17 \mathrm{keV}$ (mirror cut-off). The experiments were performed around the optimal energy of the $18 \mathrm{~mm}$ period undulator at $\mathrm{E}=12.7 \mathrm{keV}$ $(\lambda=0.97 \AA)$. The monochromaticity was defined by a double $\mathrm{Si}(111)$ monochromator. The beamline's Kirkpatrik Beaz mirror pair was used to focus the beam to approximately $7 \mu \mathrm{m}$ at the sample. The focal intensity was approximately $2.62 \cdot 10^{11}$ cps. The Mar 165 CCD detector (Mar USA, Evanston, IL), was used rebinned to $1024 \times 1024$ pixel, and a pixel size of $157.88 \mu \mathrm{m} \times 157.88 \mu \mathrm{m}$ (rebinned). NIST $\mathrm{Al}_{2} \mathrm{O}_{3}$ powder was used for calibration, see section 3.3.3. For further details see [77]. 


\subsubsection{Microfocus Beamline ID13 equipped with CRL-Optics}

Within the year between the two experiments described in chapter 8 and 9 the beamline's optical devices changed. The KB-optic was replaced by compound refractive lenses (CRL) [78]. This setting provided a much cleaner beam but simultaneously increased the focal spot from $7 \mu \mathrm{m}$ to $10 \mu \mathrm{m}$. Additionally absorption in the CRLs leaded to a reduced flux so that the illumination time for a pattern was increased from $0.5 \mathrm{sec}$ with KB-optics to $10 \mathrm{sec}$ with CRLs. The sample-todetector distance was also adapted to the position of the focal spot to approximately $123 \mathrm{~mm}$. Again the experiments were performed around the optimal energy of the undulator at $\mathrm{E}=12.7 \mathrm{keV}(\lambda=0.97 \AA)$ and the monochromaticity was defined by the double $\mathrm{Si}(111)$ monochromator. Settings and calibration of the Mar 165 CCD detector (Mar USA, Evanston, IL) were equal to those described in the previous section 3.3.1.

\subsubsection{Raw Data Treatment and Calibration}

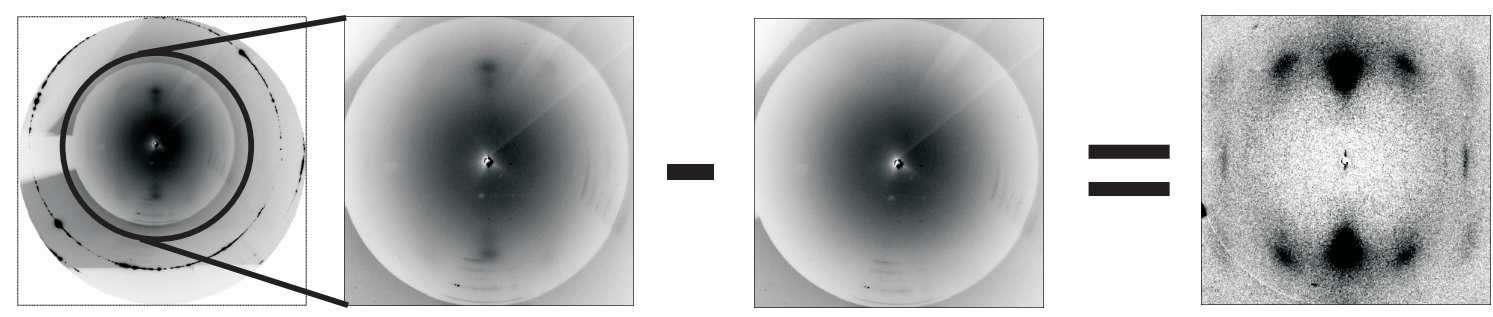

Figure 3.7: Schematic of the data treatment. From the left to the right: raw image (dark current subtracted), zoom into the raw image, background (zoom and dark current substracted), subtracted image. Without the subtraction of the background the signal is to weak for analysis.

For both setups at ID13 the data treatment was the same. Dark current, background image and sample image were collected for the same illumination time. The dark current was subtracted from both background and sample image and Miller indices were allocated to the Bragg reflections, see figure 3.7. For dragline silk of Nephila, which belongs to the Warwicker system 3 [42], the indexing is shown in figure 3.9. To allocate the reflections to a position in reciprocal space the detector was calibrated with NIST $\mathrm{Al}_{2} \mathrm{O}_{3}$ powder and the FIT2D free software by A. Hammersley [79]. $\mathrm{Al}_{2} \mathrm{O}_{3}$ powder is implemented in the Fit2D software. E. g. for the meridional plane the allocation is given by the following equations and figure 3.8:

$$
\begin{aligned}
|\vec{q}| & =\frac{4 \pi}{\lambda} \sin \left[\frac{1}{2} \operatorname{atan}\left[\frac{\left(p_{n}-p_{0}\right) \cdot p_{s}}{d}\right]\right] \\
\delta q & =q-q_{\perp}=q(1-\cos \theta) \\
\frac{\delta q}{q} & =(1-\cos \theta)
\end{aligned}
$$

With $\lambda=$ wavelength $[\AA], p_{n}=$ pixel number $n, p_{0}=$ pixel number of the primary beam, $p_{s}=$ pixel size $[\mathrm{mm}]$ and $d=$ distance sample to detector $[\mathrm{mm}]$. Where $\vec{q}$ 
is the scattering vector obtained from the triangle formed by incident beam and reflection position on the detector and $q_{\perp}$ is the $q$-value perpendicular to the fiber axis, see figure 3.3. The deviation $\delta q / q$ is approximately $1.2 \%$ for $q=2 \AA^{-1}$ and the reciprocal width of such a pixel is approximately $7 \cdot 10^{-3} \AA^{-1}$. All reflections analysed have $q$-values smaller then $q=2 \AA^{-1}$ and therefore $|\vec{q}|=q_{\perp}$. The same is true for the equatorial reflections and reflections which are neither equatorial nor meridional.To use the Fit2D calibration wavelength, sample to detector distance and position of the primary beam have to be roughly known (e.g. by calculation from equation 3.26) and are refined by the software. Simultaneously with the calibration deviations from the ideal setup geometry like detector tilt etc. were corrected.

After indexing and calibration the reflections' positions, angular and radial widths were determined as described in the next section.

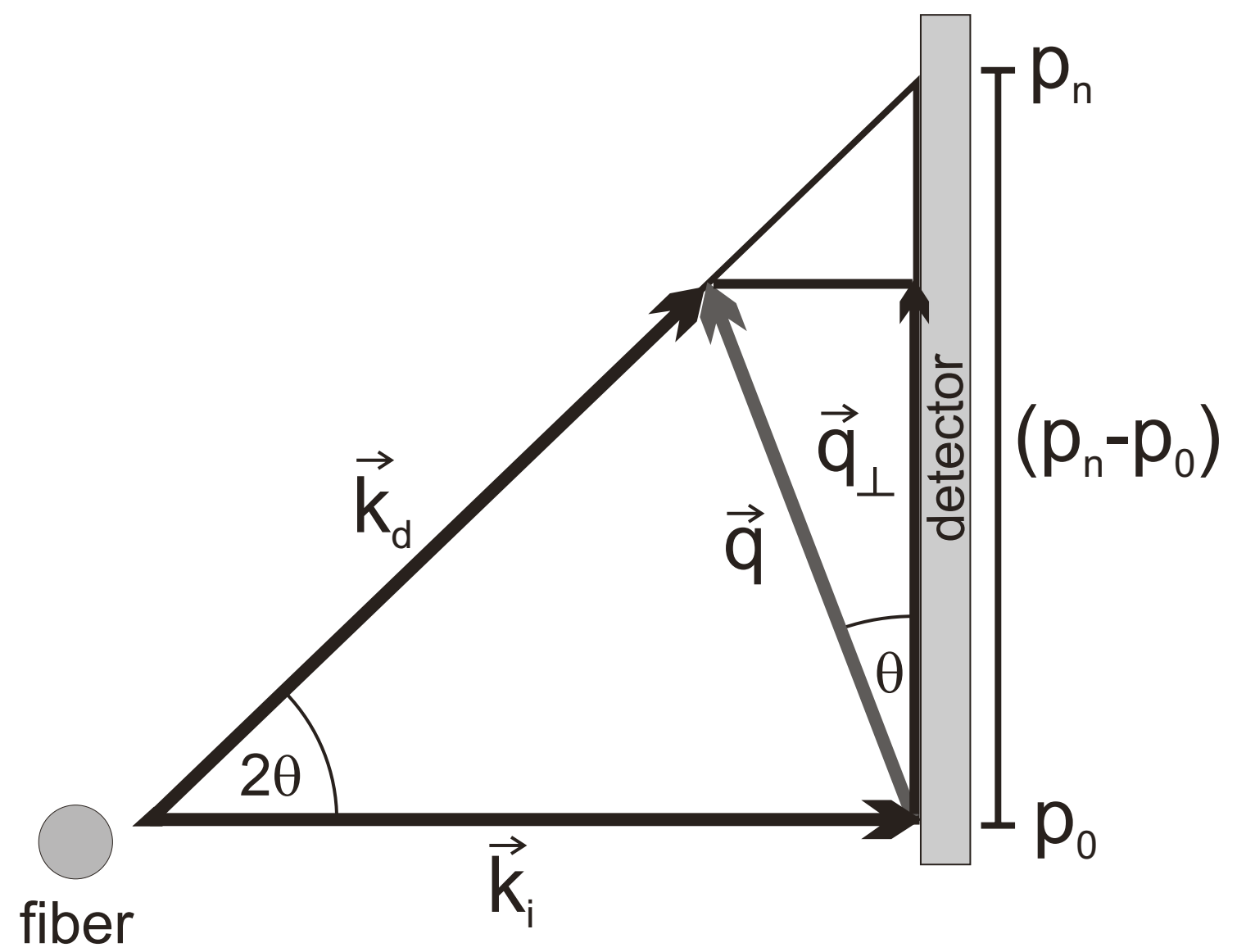

Figure 3.8: Allocation of detector signal to reciprocal space with $p_{0}=$ pixel number of the incident beam $\vec{k}_{i}, p_{n}=$ pixel number of the diffracted beam $\vec{k}_{d}$. 


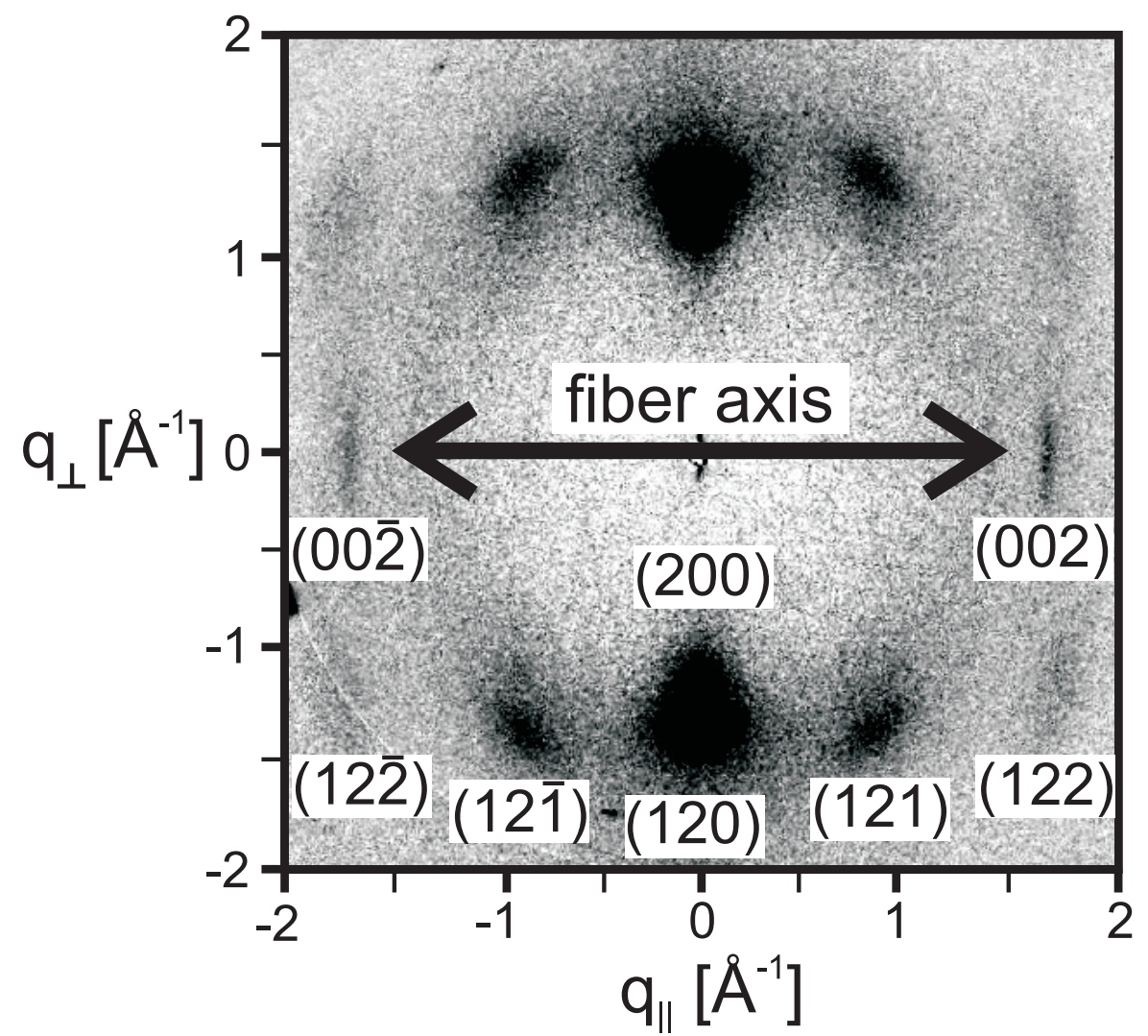

Figure 3.9: 2D-Diffraction pattern of a NM single fiber with indexing. The reflections are symmetric with respect to the fiber axis. 


\subsection{X-Ray Data Analysis}

The analysis of a 2D-diffraction pattern is best done by 1D-slices along the main symmetry axes. Such slices permit a more accurate and faster adaptation of the intensity profiles than fitting the entire 2D-diffraction pattern. To obtain onedimensional intensity profiles the slice functions and cake integration of Fit2D were used. The macros are given in appendix $C$. The different types of slices and their typical intensity profiles for dragline silk are shown in figure 3.10.

For the analysis of the intensity profiles two slightly different settings were used:

1. A range around the peak or peaks of interest was selected and fitted by Lorentzian curves with Origin 7.0 (MicroCal). In this case the amorphous halo was treated as a linear underground.

2. The fitting interval covered both peaks and amorphous halo. Peaks and amorphous halo were fitted by Lorentzian curves with Origin 7.0 (MicroCal).

The first one is more suitable for noisy profiles while the second works well for integrated and therefore less noisy ones. Both settings lead to similar results. A detailed line shape analysis of the diffraction peaks indicates that Lorentzians are better suited in describing the tails of the peaks as compared to Gaussians lineshapes. For example, in the case of the NM curves at $T=52^{\circ} \mathrm{C}$, chapter 7 , the fitting yields $\chi^{2}=1.21$ for the double Lorentzian, and $\chi^{2}=5.17$ for the double Gaussian. We therefore chose the Lorentian profiles for fitting the data although the modelling of crystalline reflections with Gaussians is more common, see figure 3.11. Importantly, the results on the peak position indicate that the mean deviation between the two models is small, for example for the (120)-reflection the relative error is smaller than $1 \%$ for the peak position, and smaller than $10 \%$ for the crystallite size, see figure 3.12. For further analysis peak position, fwhm, intensity $(I)$ and the area under the peak $(A)$, also denoted as integrated peak intensity, were recorded. With these values the slices hold the following information:

\section{Meridional and Equatorial Slices}

These two slices are the most important of the ensemble. With the peak's position $q_{h k l}$ in reciprocal angstroms the $d$-spacing of the crystallites' Bragg planes and lattice constants can be calculated, see also appendix 3. From the reflection's fwhm the crystallite size can be calculated via the well-known Debye-Scherrer formula 3.21, page 29. Additionally with a combination of crystallite size $L$ and intensity $I$ and of crystallite size $L$ and peak area $A$, relative changes in the number of scattering crystallites $N$ can be observed $\left(I \sim N L^{2}\right.$ and $\left.A \sim N L\right)$.

\section{Arc Slices and Angular Width}

From circular slices, as shown in figure 3.10c, where the circle's center and beam center are identical and the (120)-reflections's maximum is defined as outer ra- 
dius, an angular distribution of the intensity is obtained. This intensity distribution reflects the orientational distribution of the $\beta$-sheet crystallites along the fiber axis. Obviously, the smaller the angular width, the better the degree of ordering. Such profiles are best fitted by Gaussian curves because the order distribution follows mainly a Gaussian bell curve. The lower limit of the angular width is given by the instrumental peak broadening which can be estimated by the divergency of the primary beam which is negligible in respect to the broadening by the orientational distribution (mosaicity).

\section{Off-Axis Slices}

For spider silk off-axis slices are necessary because the (121)-reflection is much stronger than the (002)-reflection. Slices through the (120)- and (121)-reflection give the lattice constant along (001) and were used to check the results from the equatorial slices. The off axis slice's fwhm is not related to the crystallite size via Debye-Scherrer formula. This is only the case for radial slices through beam center and peak center.

\section{Cake Integration}

The intensity profile obtained from an integration along a circular section of $\pm 15^{\circ}$ around the (002)-reflection is suitable for the analysis of the amorphous halo. The intensity of the amorphous halo is low and noisy. This is overcome by integrating the intensity of a circular section of $\pm 15^{\circ}$ around the (002)-reflection. With bigger integration angles and width, spurious peaks contribute to the intensity profile. More points contribute to the intensity of the outer parts of the profile than to the inner ones. This can be corrected by a factor proportional to $2 \pi r$. This is automatically done by Fit2D if the option "intensity conservation" is activated. Cake integrations can also be used to analyse the (002)-reflection. 


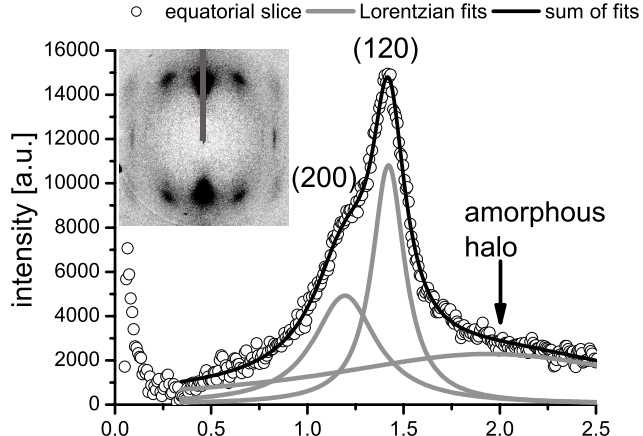

a

q $\left[\AA^{-1}\right]$

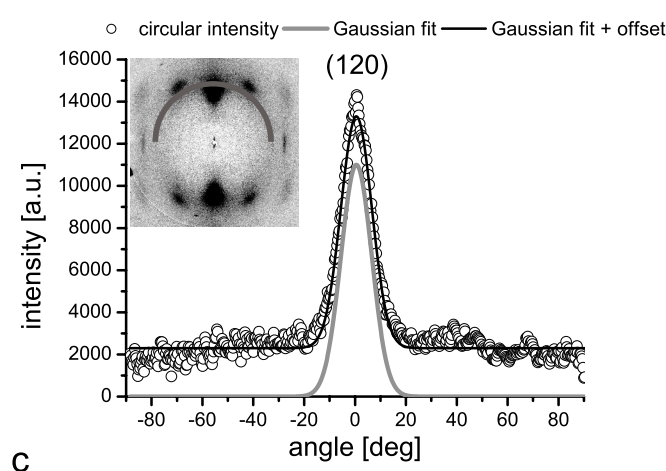

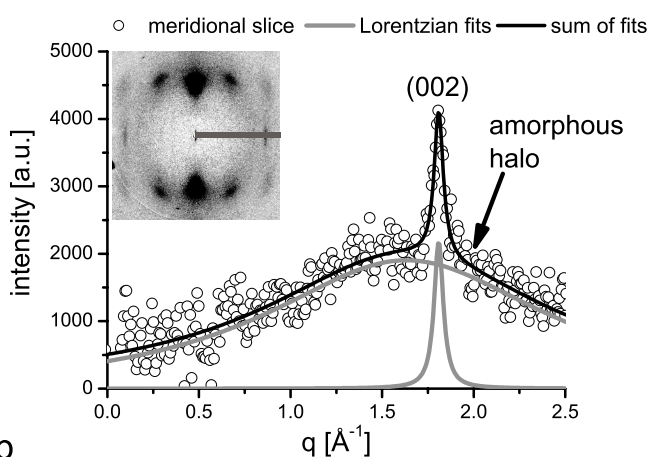

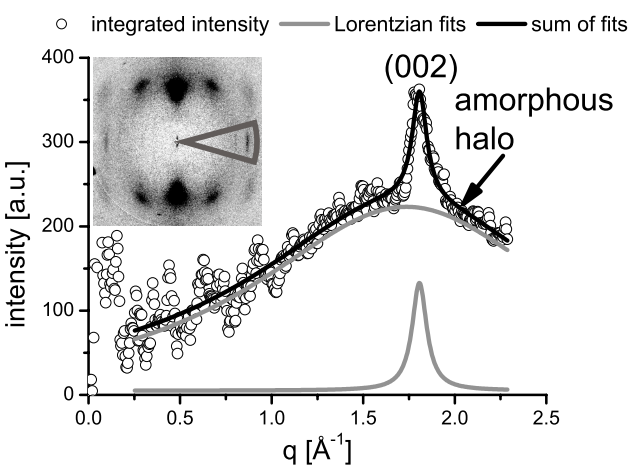

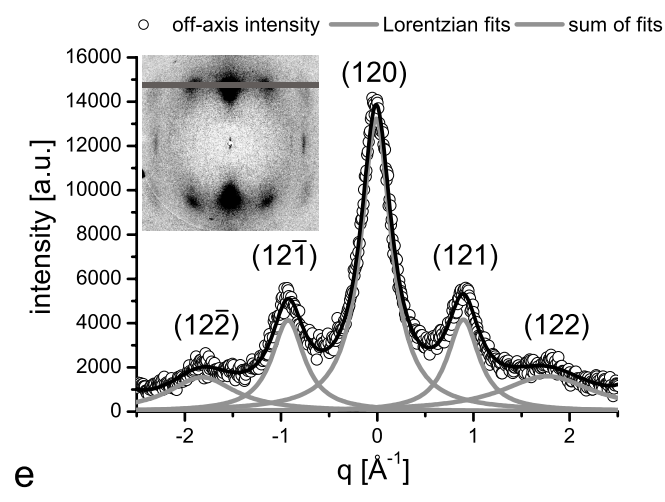

Figure 3.10: The intensities and fits along different slice and integration types used for analysis. For the angular intensity distribution a Gaussian curve was used. The other peaks were fitted by Lorentzian curves. (a) Equatorial slice through the main reflections (200) and (120). (b) Meridional slice through the (002)-reflection. (c) Circular slice through (120). (d) Integrated intensity showing the maxima of the amorphous halo and the (002)-reflection. (e) Off-axis slices through the (121)-peaks. 


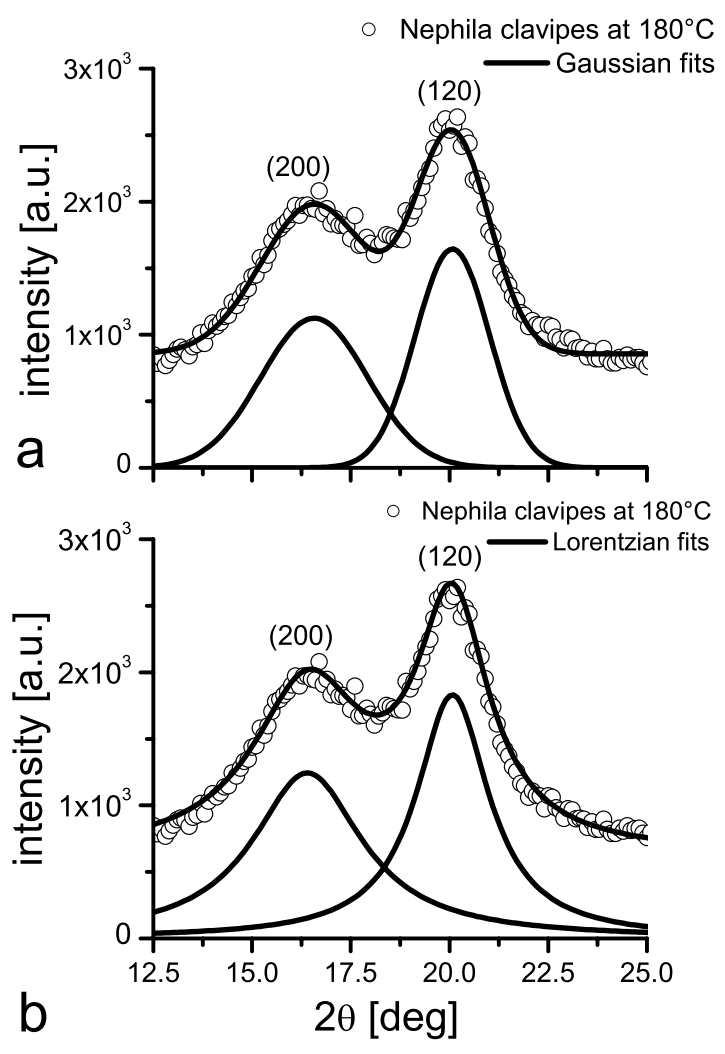

Figure 3.11: Comparison between Gaussian and Lorentzian fit. (a) Gaussian fit of the two meridional peaks of $N C$ at $180^{\circ} \mathrm{C}$, the tails of the experimental curve are not described well by the Gaussians. (b) Lorentzian fit of the two meridional peaks of $\mathrm{NC}$ at $180^{\circ} \mathrm{C}$, the tails of the experimental curve are well described. Details on the temperature dependence are given in chapter 7.

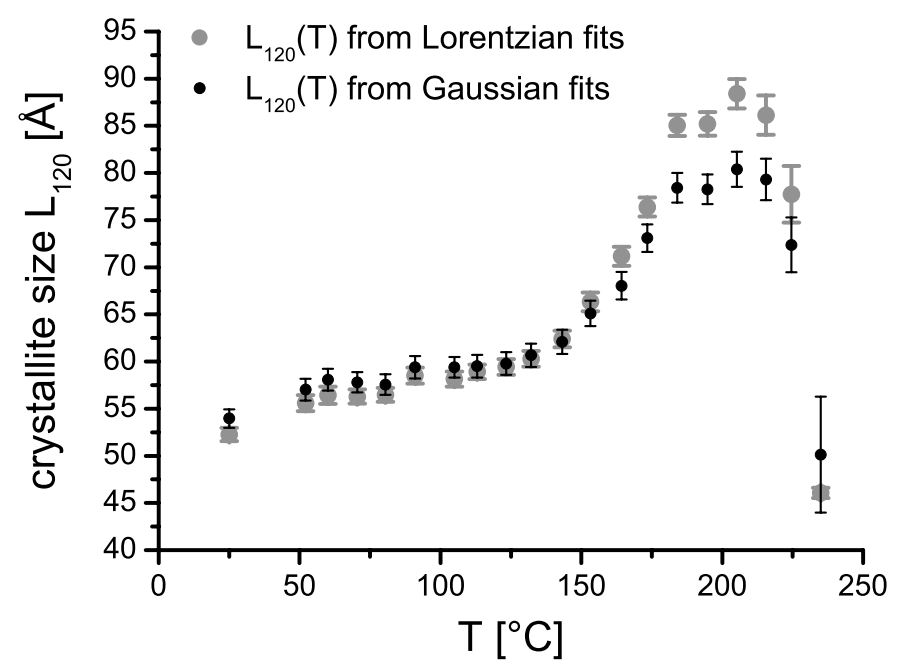

Figure 3.12: Crystallite size $L_{120}$ of NM fibers derived from Gaussian and Lorentzian fits versus temperature. Between room temperature and $150^{\circ} \mathrm{C}$ the results correspond well with each other. Between $150^{\circ} \mathrm{C}$ and $225^{\circ} \mathrm{C}$ deviations can be observed. Details on the temperature dependence are given in chapter 7 . 


\subsection{LEX 810 High Resolution Extensometer}

In our experiments single fiber diffraction was combined with simultaneous force-strain measurements. For this purpose a LEX 810 high resolution extensometer was used, as the tensile studies described in chapter 5. Together with an UV1000 control unit and an UvWin PC applications software the LEX 810 is a complete system to measure material failure at small strain values. The whole setup was developed and provided by Dia-Stron Ltd., Andover, England. Basically the extensometer consists of an optically encoded DC micrometer drive with 0.1 microns positional repeatability and a semi-conductor strain gauge load cell (Sensotec, USA) with $2.5 \mathrm{~N}$ maximum force and $5 \cdot 10^{-5} \mathrm{~N}$ force resolution, see figure 3.13. The extension rate was selectable in a range of $0.01-60 \mathrm{~mm} / \mathrm{min}$. Standard setting was $0.1 \mathrm{~mm} / \mathrm{sec}$. Three sample sizes $(7.5 \mathrm{~mm}, 15 \mathrm{~mm}$ and $30 \mathrm{~mm})$ were available. Samples of $7.5 \mathrm{~mm}$ were used for normal stress-strain measurements. In combination with X-ray diffraction samples of $30 \mathrm{~mm}$ were used to provide enough room for the alignment. The LEX810 was operated by a modified UvWin PC application to allow pauses in the stress-strain measurements to take diffraction patterns. The force-strain curves were analysed as outlined in chapter 2.3.

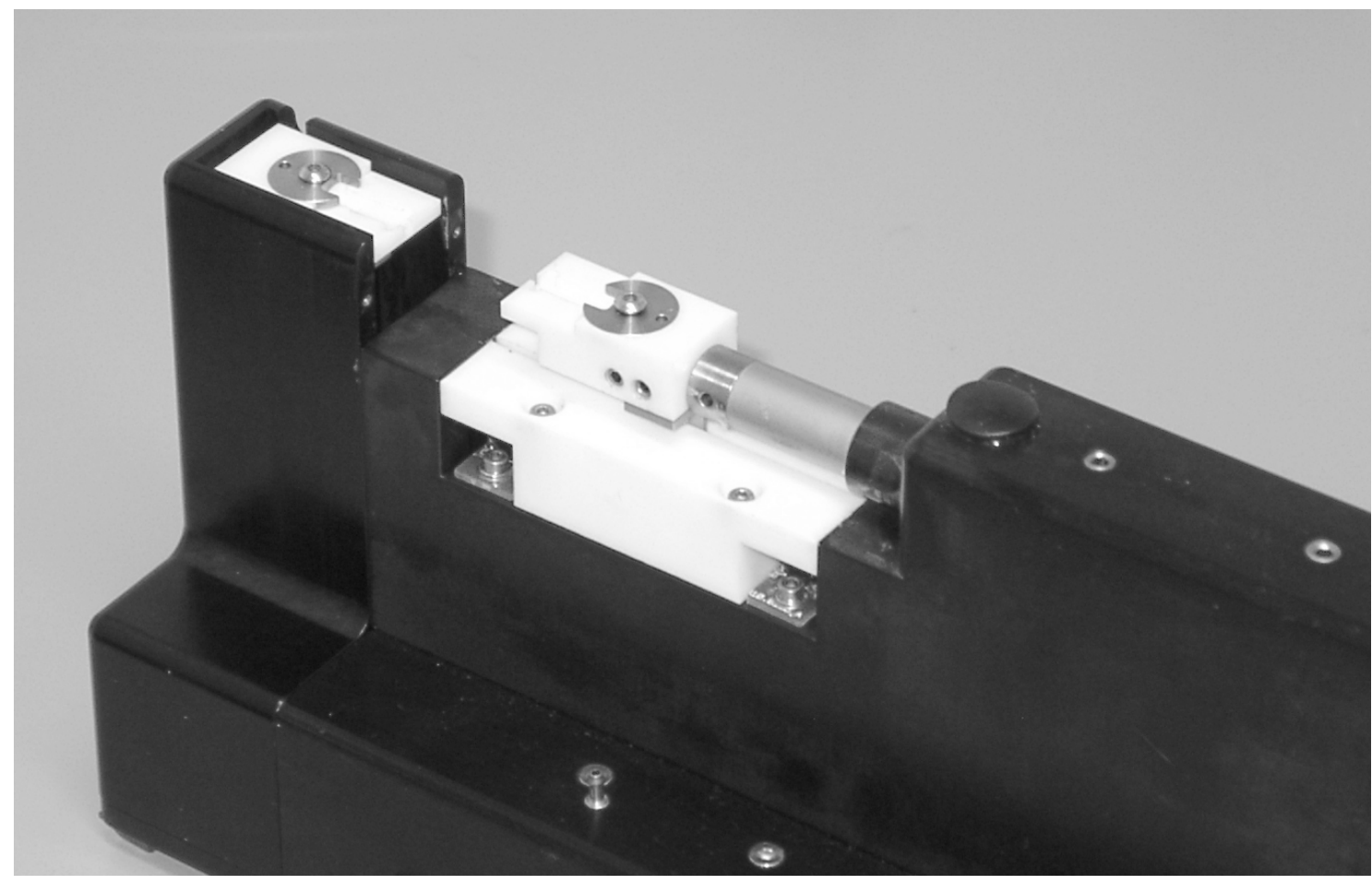

Figure 3.13: Detail of the LEX 810 high resolution extensometer. The sample pits are made from teflon. On the left the piezo load cell and on the right the optically encoded linear motor can be seen (gray tube). The load cell is removable so types of different sensitivity can be used. Available in the Institut für Röntgenphysik are a $2.5 \mathrm{~N}$ and a $20 \mathrm{~N}$ maximum force load cell. The whole instrument is about $30 \mathrm{~cm}$ long and about $8 \mathrm{~cm}$ wide and $12 \mathrm{~cm}$ high. 


\subsubsection{Humidity Chamber}

In order to study the dependency of structure and mechanical properties on the relative humidity (RH) a chamber for the LEX 810 was designed with respect to the requirements of single fiber $X$-ray diffraction. Basically the humidity chamber is a housing for the extensometer's entire sample area, welded from $2 \mathrm{~mm}$ steel plates. Two $80 \mathrm{~mm} \times 40 \mathrm{~mm}$ windows of 4 micron polypropylene foil as beam pass and a $6 \mathrm{~mm}$ tube housing for humid nitrogen were integrated. The chamber is not hermetic, so no pressure control valve is necessary. The nitrogen was provided by the institute's and the beamline's pressurized nitrogen supply. Directly from the supply the nitrogen has a humidity of less than 5\% RH. Humidities around 95\% could be achieved by passing the nitrogen through a water-filled gas-washing bottle. The size of the gas bubbles is essential for the degree of humidification. The bigger the bubbles, the lower the humidity of the exiting gas stream. The bubbles' size was controlled by a filter in the gas-washing bottle and a moderate flow. Other humidities were set by mixing an arid and a humid gas stream. The gas flow was controlled by tunable valves and the humidity was checked with a testo 635 humidity and temperature sensor (Testo AG, Lenzkirch, Germany) integrated into the tubes, see figure 3.14.

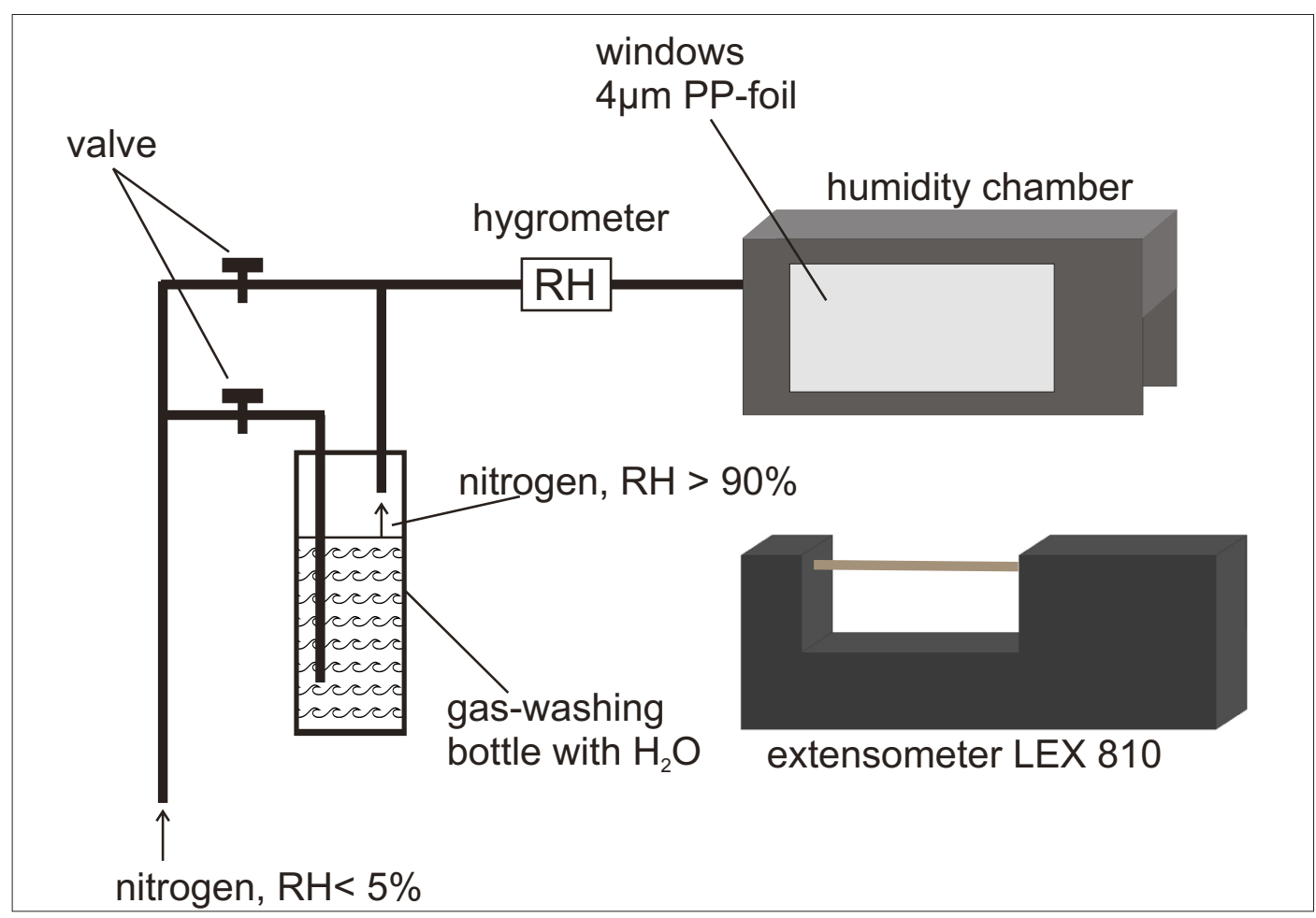

Figure 3.14: Schematic of the experimental setup with humidity control. The humidity chamber can be imposed on the extensometer. The windows for the X-ray beam are $4 \mu \mathrm{m}$ polypropylene (PP) foil. For humidities below 5\% RH pressurized nitrogen from the beamline's supply was used directly. For higher humidities dry nitrogen and nitrogen moistened by passing through a water-filled gas-washing bottle were mixed. The humidity was controlled by a testo 635 humidity and temperature sensor inserted before the humidity chamber. For humidities over $90 \%$ RH pure moistened nitrogen was used. 


\section{Chapter 4}

\section{Forced Silking and Samples Preparation}

\section{The Genus Nephila}

Females of three different species of the genus Nephila have been kept in the institute, namely Nephila senegalensis (NS), Nephila clavipes (NC) and Nephila madagascariensis (NM), see figure 4.1. The spiders lived in wood frame cages of $40 \mathrm{~cm} \times 40 \mathrm{~cm} \times 20 \mathrm{~cm}$ size. The small sides are made of a $2 \mathrm{~mm} \times 2 \mathrm{~mm}$ mesh and equipped with a flap for feeding. Back and front are made of plexiglass and totally removable for catching the inhabitant. The cages were stored in a glass cabinet at $22^{\circ} \mathrm{C}$ night temperatures and $26^{\circ} \mathrm{C}$ by day. The humidity varied between $50 \%$ to $70 \%$ depending on the ultrasonic vaporizer's and the radiator's working intervals. The spiders were fed with Calliphora (blowflies) every one or two days. The web was wetted daily with sprayed water.

The dragline samples were collected by a variation of forced silking [80]. The spiders were sedated in the refrigerator for $1 \frac{1}{2}$ hours at temperatures between $3^{\circ} \mathrm{C}$ and $5^{\circ} \mathrm{C}$. For some spiders it was necessary to sedate them at $0^{\circ} \mathrm{C}$ because at higher temperatures the immobilization was incomplete. For this purpose the spider was moved into an smaller plastic box $(10 \mathrm{~cm}$ in diameter and $13 \mathrm{~cm}$ heigh, vent holes only in the lid), which was placed in a mixture of water and ice. Again this setup was left for $1 \frac{1}{2}$ hours in the refrigerator. We interpret the phenomena of cold resistance as an adaptation because it often occurred with spiders bred in Europe for some generations. After sedation the spiders were fixed up side down on a piece of styrofoam to expose the spinning apparatus, see figure 4.2.

Sometimes a longer dragline thread was still existent and could be picked up with forceps. If this was not the case, the spinnerets had to be stimulated with a soft brush. In a knee-jerk reaction the spider attaches a mixture of different silk types to the bristles. The dragline had to be separated from the other types of silk threads. This can easily be done optically. The dragline fibers are from the major ampullate gland and the thickest type of all the silks an orb-web spider produces, see section 2. A binocular microscope at 10x magnification was used for this and to separate the two dragline fibers from the pair of major ampullate glands every Nephila spider has, if single fibers were desired. The fibers were wound directly onto a sample holder or onto a reel for storage. The fibers are best stored in the 
dark at ambient conditions. The maximum frequency for silking was one time per week and spider. The amount of harvested threads varied between $60 \mathrm{~m}$ and $120 \mathrm{~m}$ depending on the spiders condition. It was checked that fibers collected in this way and fibers collected directly from the web showed no differences in thickness by optical microscopy.

\subsection{Degumming}

In the native state the silk fibers are surrounded by a sericin coat which can have a thickness up to $10 \%$ of the fiber's diameter [49]. Sericin is a hygroscopic glycoprotein which ensures the constant humidity of the fiber and acts as an adhesive between double fibers. The sericin coat can be removed by washing in a slight alkaline solution, e.g. a mixture of common commercial washing agent and water as used for textiles. Normally $40 \mathrm{~min}$ at $60-70^{\circ} \mathrm{C}$ are sufficient to remove the whole sericin coat. Afterwards the fibers have to be cleaned in a water-bath (1h at $30^{\circ} \mathrm{C}$ ). It is useful to process the fibers on the reel which has to be resistant against the alkaline solution. This was the case for the used PE-reels. For X-ray diffraction and mechanical measurements it is not necessary to remove the sericin coat but it is useful to obtain single threads from double fibers when they could not be obtained during forced silking.

\subsection{Sample Preparation}

For the temperature dependent studies on fiber bundles the fibers were directly reeled onto the sample holder. For single fiber diffraction experiments in combination with stress-strain measurements and normal stress-strain measurements the fibers had to be trimmed and fixed on a special sample holder. In principal the sample holders are rectangular plastic items with two $0.5 \mathrm{~cm}$ deep cups at both ends for the glue. The cups have a small depression on the edge to prevent a bending of the sample. The sample holders are positioned in trays with predefined distances of $7.5 \mathrm{~mm}, 15 \mathrm{~mm}$ and $30 \mathrm{~mm}$. Such a tray again is placed on a adjustable vacuum tray. The suction helps to place the fiber in the sample holders and fixes it during application and hardening of the glue. The Dymax 3094 and 3013 UV/VIS-adhesives (Dymax Europe GmbH, Frankfurt a.M., Germany) were hardened for five seconds by a CoolLED 2600 UV Curing Pen (COOLLED Ltd., Andover, UK). The samples were transfered into the extensometer by a vacuum tweezer which ensured that the samples were kept at the predefined length and not strained in any way. The whole sample preparation items were provided together with the LEX 810 High Resolution Extensometer by Dia-Stron Ltd. (Andover, UK). 


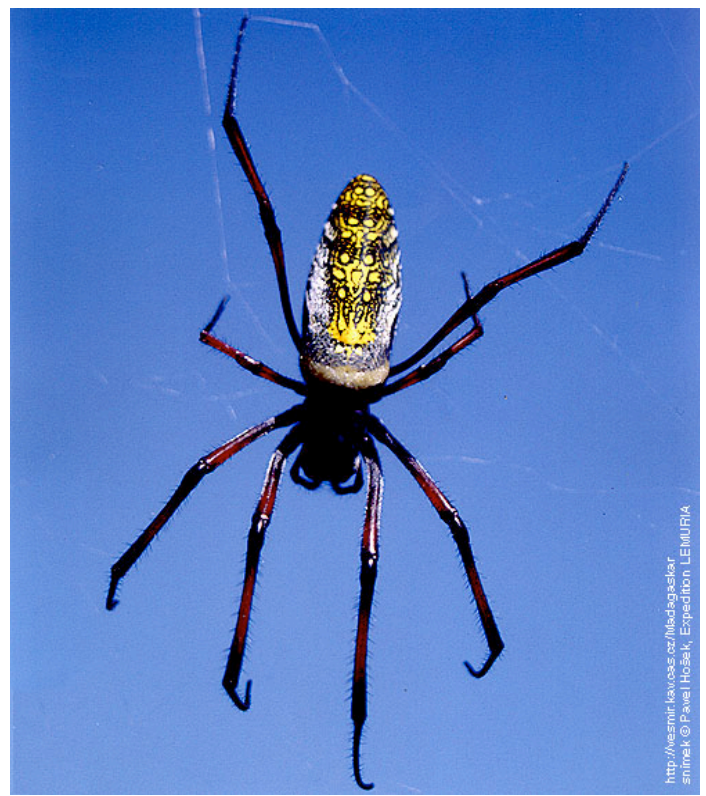

(a) N. madagascariensis

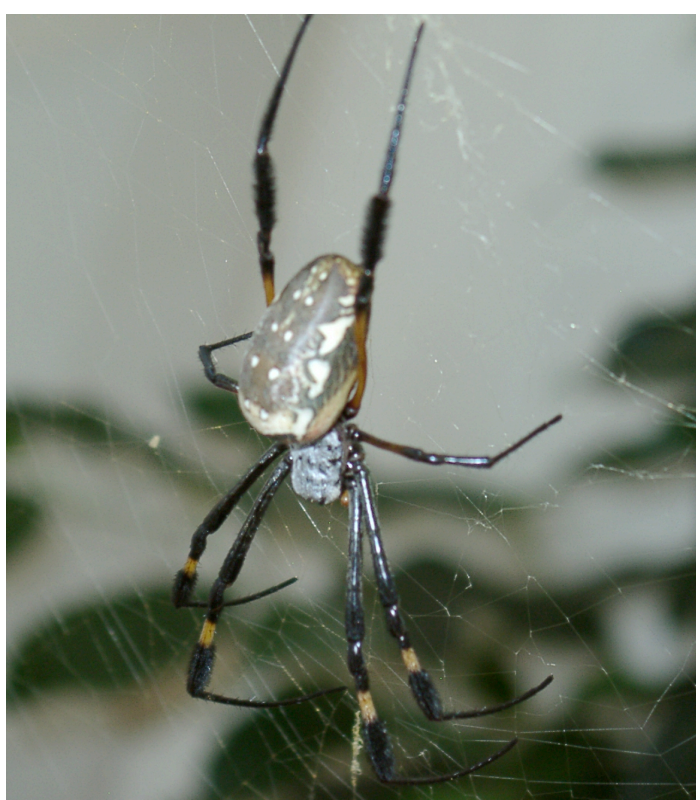

(b) N. senegalensis

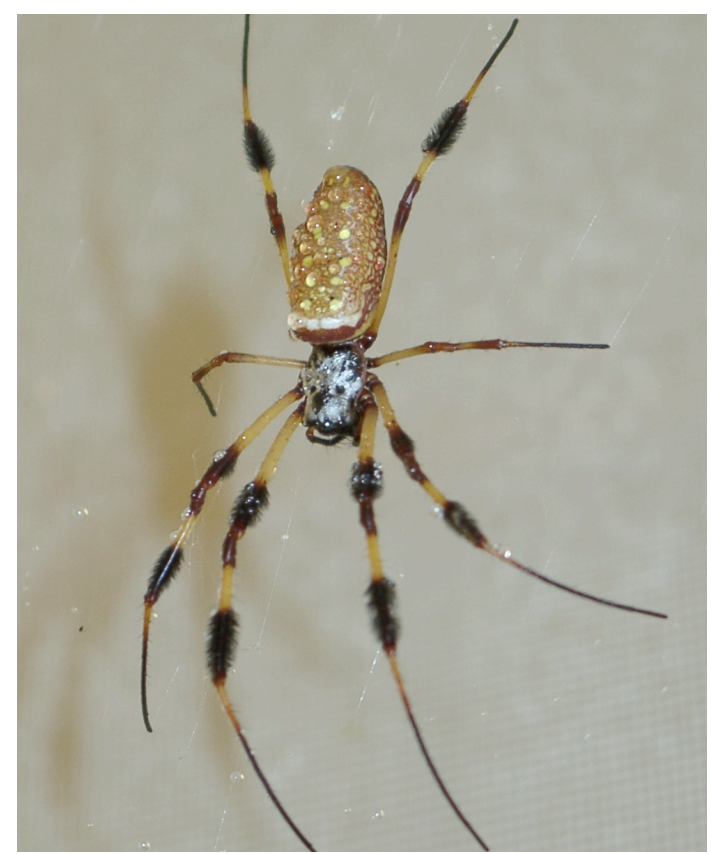

(c) N. clavipes

Figure 4.1: The three Nephila species kept in the Institut für Röntgenphysik. The genus Nephila inhabits tropical and subtropical regions around the world. NC originates from Florida while NS and NM are from West and East Africa, respectively. The picture of NM is by Pavel Hošek, Expedition Lemuria, the pictures of NS and NC were taken at the Institute für Röntgenphysik. 


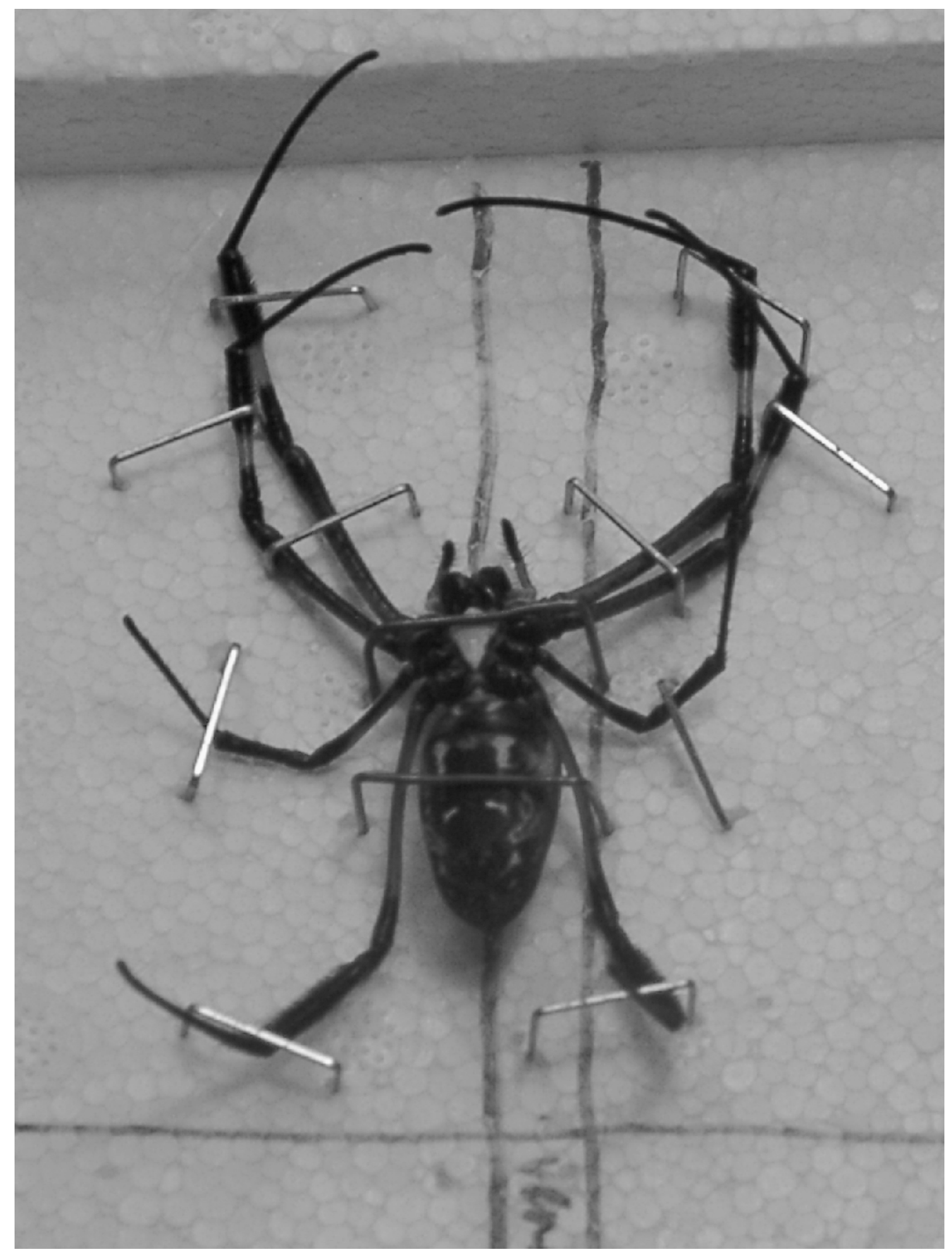

Figure 4.2: A female NS spider fixed for silking. The high number of clamps is necessary to prevent the spider from retracting her very movable legs from the fixation. The clamps work by blocking the legs' degrees of freedom and have to be fasten by hand to avoid any injuries of the spider. The ladies were silked with a maximum frequency of once a week. 


\section{Chapter 5}

\section{Dragline Silk: Humidity, Hysteresis and Relaxation}

Related to this doctoral thesis two diploma theses were carried out at the Institute für Röntgenphysik. The work of Thorsten Vehoff [52; 53] focused on a detailed analysis of the influence of humidity on dragline's mechanical properties and will be outlined here. The other work dealt with scattering simulations. Humidity has a strong influence on the mechanical properties of untreated polyamides [81; 82] to which dragline belongs in the broadest sense [66]. In particular this can be seen in supercontraction of wet dragline threads [54; 55; 56] (chapter 2.4) and the two different forms of stress-strain curves for dragline silk described in literature. One is of sigmoidal shape [83], the other consists of two linear regimes with a yield point in between [39]. The differences in the shape form are rarely discussed in literature, as are the circumstances at which the measurements were performed. For this purpose more than hundred MAS fibers from N. clavipes and $N$. senegalensis were studied at four different humidities: $25 \%, 44 \%, 75 \%$ and $85 \%$ $\mathrm{RH}$. Breaking strain $\varepsilon_{\max }$, breaking force $F_{\max }$, the energy the fiber can take prior to breaking $E$ as well as the initial elastic modulus $\mathcal{E}$ were determined at these conditions, using the linear extensometer LEX 810 described in chapter 3.5.

To set the different humidities a special humidity chamber was adapted to the extensometer. It was made of Teflon and comparable to the chamber used for the diffraction experiments, chapter 3.5.1, but additionally hold two reservoirs for salt solution. For relative humidities of about $25 \%$ a steady flow of nitrogen was used, $44 \%$ RH corresponded to the laboratory's standard conditions, 75\% RH was obtained by saturated water sodium chloride $(\mathrm{NaCl})$ solution and $85 \%$ by pure water in the reservoirs. Temperature and humidity were measured by a testo 635 sensor (Testo AG, Lenzkirch, Germany). Further details can be found in section 3.5 .1 and in $[52 ; 53]$.

\subsection{Influence of Humidity}

At humidities below 35\% RH the stress-strain curve of dragline consists of two linear regimes. For rising humidity the curve's shape changes by a decreasing initial slope followed by a more pronounced plateau to the sigmoidal curves and 
ends at humidities above 75\% RH in an elastomeric shape, as shown in figure 5.1. With increasing humidity the fiber softens (decrease of the initial elastic modulus $\mathcal{E})$ and extensibility increases $\varepsilon_{\max }$. The extensibility $\varepsilon_{\max }$ was found to increase with increasing humidity by $\varepsilon_{\max }[\%]=(0.29 \pm 0.03) \cdot R H+(13.1 \pm 1.5)$ while the elastic modulus $\mathcal{E}$ deceases by $\mathcal{E}[\mathrm{GPa}]=-(0.185 \pm 0.008) \cdot R H+(17.0 \pm 0.6)$. The increased extensibility was explained by the phenomena of supercontraction, mentioned in chapter 2.4. Analogous to polyamides it is most likely caused by water molecules permeating into the amorphous matrix. The water substitutes hydrogen bonds between the protein chains by forming hydration shells. This decreases the interaction between the single protein strands, they behave more like an ideal chain, the amorphous matrix softens. Further results indicate that both breaking force $F_{\max }$ and stretching energy $E$ are nearly the same for humid and arid fibers, see tables 5.1 and 5.2. This indicates that for rupture the same amount of covalent protein bonds has to be broken for both states (arid and humid), see tables 5.1 and 5.2.

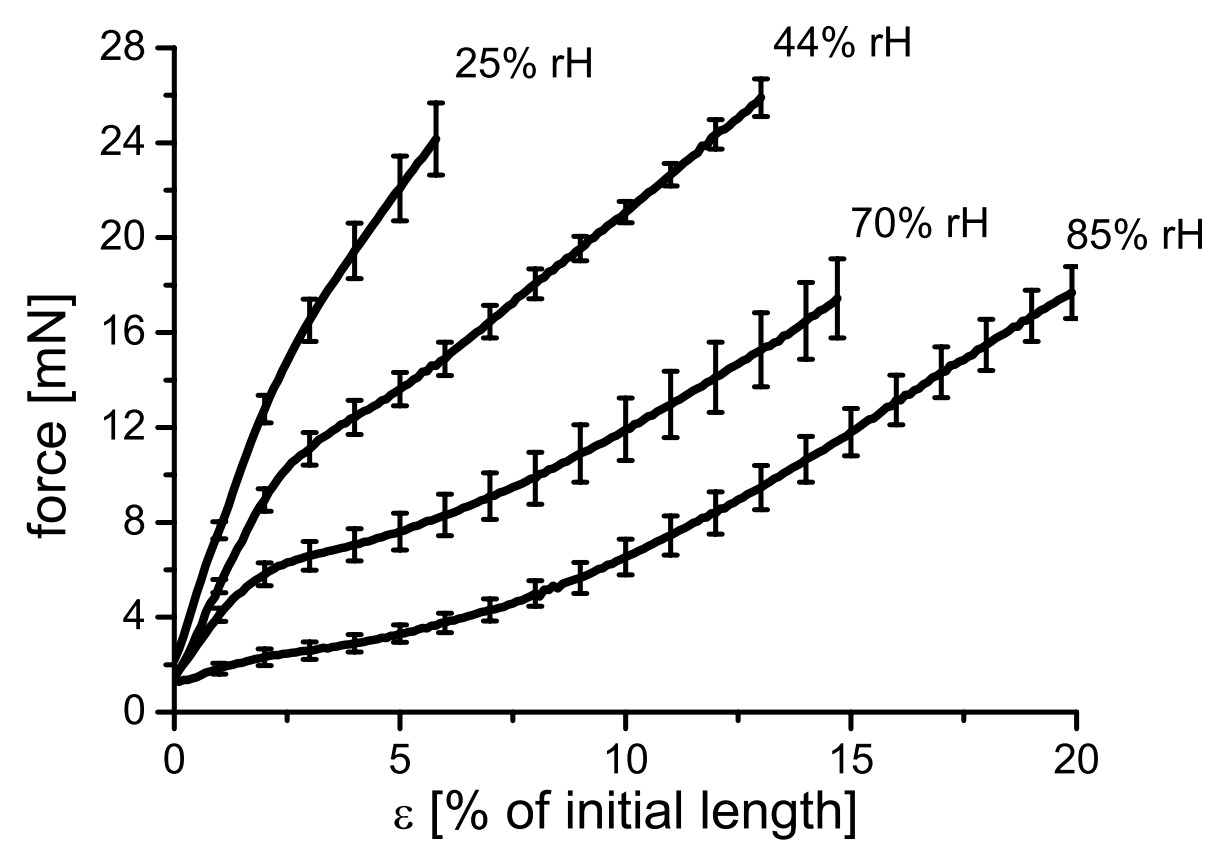

Figure 5.1: Influence of humidity on the tensile behavior of NS dragline. With increasing humidity extensibility increases and the slope decreases. The curves shown are the mean curves of the ensembles measured at different humidities. The curves are shown up to the extension at which the first fiber of an ensemble tears (ensemble's minimal extension). Data by T. Vehoff [52; 53]. 


\subsection{Hysteresis}

The hysteresis measurements were performed between $37 \%$ and $44 \% \mathrm{RH}$. Up to ten hysteresis cycles per sample were recorded followed by a measurement until failure, see figure 5.2. The measurements were performed without a gauge force, see chapter 2.3. The energy loss in the first hysteresis cycle (area between ascending and descending curve) was found to be $68 \% \pm 2.2 \%$. The following hysteresis cycles differ in shape form the first one. The ascents are shifted closer to the descents so that the stretching energy $E_{x}$ is only approximately half the energy of the first cycle $E_{1}=2 E_{x}$ and only $37 \%$ of $E_{x}$ is dissipated. The differences between all hysteresis curves after the first is negligible, as for first and following descents, see figure 5.3 .

To extend the fiber beyond the maximum extension of the previous hysteresis cycle the force has to be greater than the force applied previously. In order to check this a second set of hysteresis measurements was performed in which a gauge force of $1.5 \mathrm{mN}$ was set (compare chapter 2.3) with the consequence that each cycle started at new, longer length. The plot of such a set of hysteresis cycles with gauge is shown in figure 5.4. The envelope of the hysteresis cycles is identical with that of a single measurement until breaking-point.

\subsection{Relaxation}

Beside the influence of humidity and hysteresis, the relaxation of elongated dragline fibers was studied $[52 ; 53]$. For this purpose the samples were extended up to $\varepsilon=20 \%$ and held in place for 300,600, 1200 and 1800 seconds while the force-time behavior was recorded. The curves found in this way were best described by a stretched exponential function: $F(t)=F_{1}+F_{2} \cdot \exp \left(-(t / \tau)^{\beta}\right.$. With $F_{1}$ denoting the force after complete relaxation, $F_{2}$ the fraction of force decreasing to zero at infinite times and $\tau$ the relaxation time. In this way it was found that it was impossible to obtain an average relaxation time. But the relaxation parameter $\beta$ could be determined to $\beta=0.320 \pm 0.033$ which indicates that many different relaxation processes and time scales are involved.

Table 5.1: Tensile properties of NS dragline at different humidities [52].

\begin{tabular}{cllll}
\hline & $25 \% \mathrm{RH}$ & $45 \% \mathrm{RH}$ & $70 \% \mathrm{RH}$ & $80 \% \mathrm{RH}$ \\
\hline$F_{\max }[\mathrm{mN}]$ & $44.4 \pm 2.7$ & $40.3 \pm 3.6$ & $35.3 \pm 2.5$ & $33.0 \pm 3.1$ \\
$\varepsilon_{\max }[\%]$ & $18.9 \pm 2.0$ & $24.2 \pm 2.8$ & $30.6 \pm 1.8$ & $34.6 \pm 2.5$ \\
$E[\mu \mathrm{J}]$ & $40.6 \pm 5.7$ & $46.0 \pm 8.9$ & $42.2 \pm 4.4$ & $39.3 \pm 6.4$ \\
$\mathcal{E}[\mathrm{GPa}]$ & $10.5 \pm 0.6$ & $7.8 \pm 0.4$ & $5.0 \pm 0.5$ & $1.4 \pm 0.5$ \\
\hline
\end{tabular}


Table 5.2: Tensile properties of NC dragline at different humidities [52].

\begin{tabular}{clll}
\hline & $25 \% \mathrm{RH}$ & $44 \% \mathrm{RH}$ & $80 \% \mathrm{RH}$ \\
\hline$F_{\max }[\mathrm{mN}]$ & $30.0 \pm 2.1$ & $33.7 \pm 1.9$ & $36.5 \pm 1.4$ \\
$\varepsilon_{\max }[\%]$ & $22.6 \pm 1.2$ & $24.6 \pm 1.5$ & $39.8 \pm 1.3$ \\
$E[\mu \mathrm{J}]$ & $32.7 \pm 3.0$ & $38.0 \pm 3.7$ & $43.7 \pm 2.8$ \\
$\mathcal{E}[\mathrm{GPa}]$ & $13.7 \pm 0.7$ & $14.0 \pm 0.7$ & $1.4 \pm 0.5$ \\
\hline
\end{tabular}

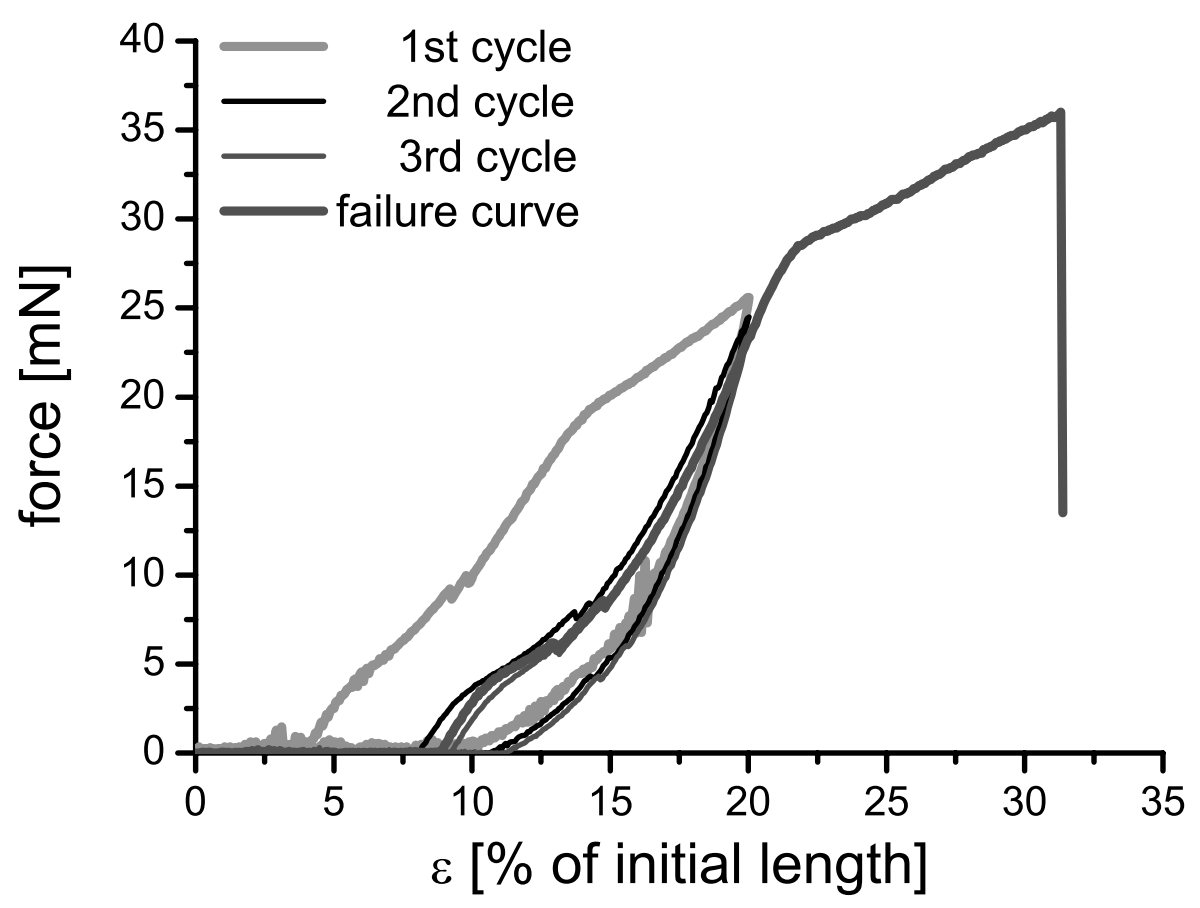

Figure 5.2: Five hysteresis cycles followed by a force-strain curve up to failure. Only the first hysteresis curve differs from the following cycles. Together the ascent of the first hysteresis cycle and the second half $(\varepsilon>18 \%)$ of the failure curve have a shape which is equal to a force-strain curve obtained without the hysteresis cycles. Data by T. Vehoff [52; 53]. 


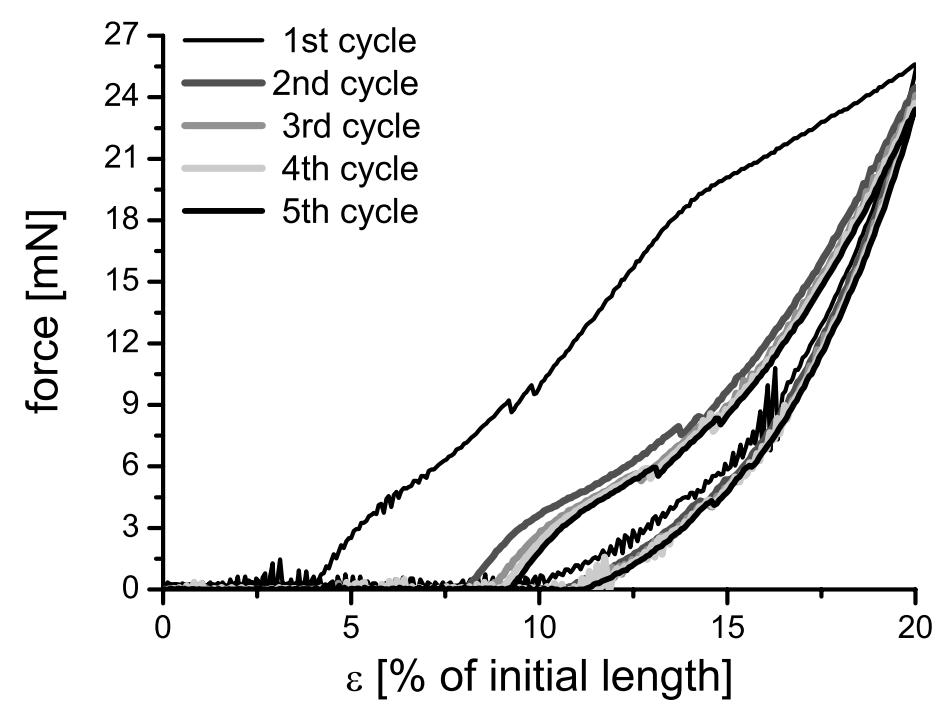

Figure 5.3: Detail of the five subsequent hysteresis cycles from figure 5.2. The curve differences after the first cycle are negligible. Data by T. Vehoff [52; 53].

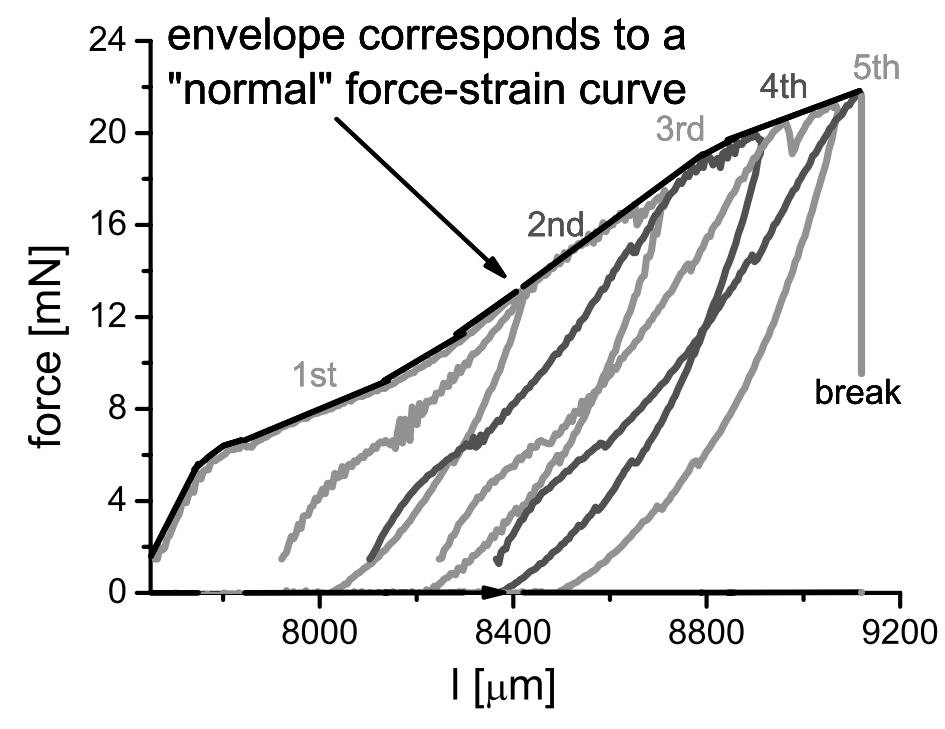

Figure 5.4: Ensemble of five hysteresis cycles with gauge force. The gauge force forces the measurement to start after a preset threshold is crossed. The cycles are shifted to higher extensions. Ascent and descent now longer merge directly into each other but are now connected by a plateau with smaller positive slope. These plateaus along with the first ascent form a stress-strain curve as found by simple stress-strain measurements. Data by T. Vehoff [52; 53]. 



\section{Chapter 6}

\section{Scattering Simulation and Crystallite Composition}

The diploma thesis of M. H. Meling [84; 85], the second work related to the spider silk project at the Institute für Röntgenphysik, focused on the construction and scattering simulation of virtual $\beta$-sheet crystallites in order to study the effect of different crystallite compositions and structures [84]. Historically the idea of comparable studies was to estimate a probable amino acid composition, to produce an artificial crystal with this composition and to compare the diffraction patterns from this crystal with that of silk, see figure 6.1. From these experiments we know that the scattering from silk is dominated by poly-L-alanine [86] and was also proved for spider silk [34].

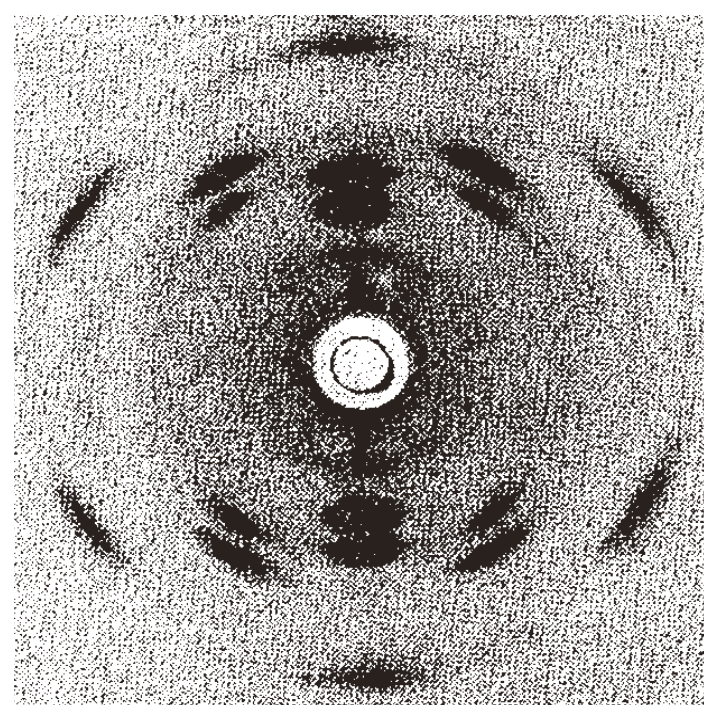

(a) Poly-L-Alanine

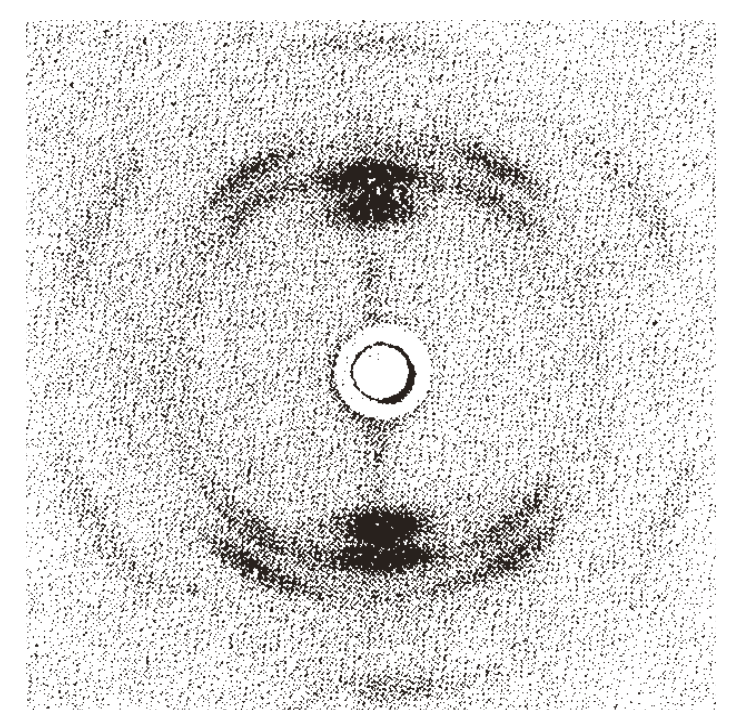

(b) Tussah Silk

Figure 6.1: Diffraction Pattern of tussah silk (silk from a wildform of the silkworm Bombyx mori) and $\beta$-poly-L-alanine. The similarity is striking, from [86].

Naturally, this method is elaborate, expensive and time-consuming. The nowadays available computing power makes it possible to study the scattering from nearly any crystal structure and to compare it with experiments. For Nephila this 
has been done by B. L. Thiel et al. [35] and for Neutron scattering by D. Sapede [87]. Thiel and co-workers introduced an alternative explanation for the structure of spider silk by proposing non-periodic lattice crystals. The diffraction of such crystal would be comparable to that of small but perfect crystals as necessary to apply the Debye-Scherrer formula, equation 3.21, page 29. This and the desire to study the effect of different amino acid compositions [88] and defects in the crystal lattice [34] were the motivation to develop our own scattering simulation program. Such an approach was tried in the first diploma thesis about spider silk written in our institute [12]. In this thesis the atom positions in the unit cell given by Corey and co-workers [41] for the silk from B. mori were expanded to a pure alanine crystallite with larger lattice constants. Although these simulations were able to reproduce the main equatorial peaks the results were unsatisfactory. It was realized that a simulation of the diffraction required more sophisticated methods and much more time. Stephan Ulrich from the Institute für Theoretische Physik and Martin H. Meling from the Max-Planck-Institut für Biophysikalische Chemie constructed and optimized virtual crystallite structures using the costume made scattering simulation program scattering $c B a y$ and the molecular dynamic simulations program GROMACS [89].

\subsection{Simulation Program $c B a y$}

Basically the simulation program cBay calculates the scattering of a given crystal as outlined in chapter 3. Additionally, the scattering function $\mathcal{S F}$ incorporates the rotary matrix $\mathcal{D}$ to allow different crystal orientations. Then the scattering function of one crystal with orientation $\mathcal{D}$ becomes

$$
\begin{gathered}
\mathcal{S F}(\vec{q} \mathcal{D})=\mathcal{S}(\vec{q} \mathcal{D}) \cdot \mathcal{F}(\vec{q} \mathcal{D})=\sum_{\vec{R}_{n}} e^{i \vec{q} \mathcal{D} \vec{R}_{n}} \cdot \sum_{\vec{r}_{j}} f_{i}(\vec{q}) e^{i \vec{q} \mathcal{D} \vec{r}_{j}} \\
=\frac{\sin \left(\frac{N_{a} \vec{q} \mathcal{D} \vec{a}}{2}\right)}{\sin \left(\frac{\vec{q} \mathcal{D} \vec{a}}{2}\right)} \frac{\sin \left(\frac{N_{b} \vec{q} \mathcal{D} \vec{b}}{2}\right)}{\sin \left(\frac{\vec{q} \mathcal{D} \vec{b}}{2}\right)} \frac{\sin \left(\frac{N_{c} \mathcal{D} \vec{q} \vec{c}}{2}\right)}{\sin \left(\frac{\vec{q} \mathcal{D} \vec{c}}{2}\right)} \cdot \sum_{\vec{r}_{j}} f_{i}(\vec{q}) e^{i \vec{q} \mathcal{D} \vec{r}_{j}},
\end{gathered}
$$

where $\vec{a}, \vec{b}$ and $\vec{c}$ are the unit cell's basis vectors, $\vec{r}_{j}$ the position of atom $f_{j}$ within the unit cell and $N_{a}, N_{b}$ and $N_{c}$ are the number of unit cells along the three crystal axes $x, y, z$, as defined in chapter 2.2 and 3 . Thus the intensity $I(\vec{q})$ for different orientations becomes

$$
I(\vec{q})=\int|\mathcal{S}(\vec{q} \mathcal{D}) \cdot \mathcal{F}(\vec{q} \mathcal{D})|^{2} p(\psi, \theta, \varphi) d \psi d \theta d \varphi,
$$

where $\psi, \theta$ and $\varphi$ are the Euler angles of the rotary matrix and $p(\psi, \theta, \varphi)$ is the probability for each angle. In the case of dragline silk $-\pi \leq \psi, \varphi \leq+\pi$ to reproduce the orientational equipartition of $x$ - and $y$-axis around the $z$-axis, while $\theta$ (deviation of $z$-axis from fiber axis) is described by a Gaussian distribution with $5^{\circ} \leq \sigma_{\theta} \leq 7.5^{\circ}$.

In a similar way it was possible to simulate the scattering from the amorphous 
matrix. In this case the equations for scattering from a crystal were replaced by those of scattering from a liquid of identical particles (amino acids) in which distance and orientation of the amino acids is totally random. By comparing such simulations with the experiment it was found that the mean distance within the amorphous matrix is approximately $3.26 \AA$. The Debye-Waller factor was also taken into account and was found to be responsible for the low intensity of reflections with $q>2 \AA^{-1}$.

\subsection{Alanine Crystallites}

The virtual $\beta$-sheet crystallites were constructed with the program YASARA [90], starting from an alanine chain with 12 amino acids ( $\widehat{=} 6$ unit cells) and an adjacent amino acid distance of $3.5 \AA$. This chain was multiplied and arranged in a parallel or anti-parallel way along the $y$-axis. Then this double strand was duplicated and translated along the $x$-axis to form a subunit with the same symmetry as the $\beta$-sheet crystallite, see figure 6.2. The lattice constants $a$ and $b$ where varied in order to allow a study of slightly different crystallites, $b$ was chosen between $9.4 \AA \leq b \leq 9.8 \AA$ and $a$ between $10.2 \AA \leq a \leq 10.8 \AA$.

By subsequent laying of these subunits the desired crystallite was constructed, e. g. an anti-parallel crystallite given by Grubb [5] has a unit cell of $10.6 \AA \times 9.44$ $\AA \times 6.95 \AA$, which are repeated $2 \times 5 \times 6$ times along the respective axes and form a crystallite $21 \AA \times 47 \AA \times 42 \AA$. Both parallel and anti-parallel crystallites have the same size.

The scattering simulations of both parallel and anti-parallel arrangement lack the (120)-reflection due to deviations from the $\beta$-sheets natural symmetry. To overcome this problem the $\beta$-strands where shifted by half an unit cell along the $y$ axes (y-shift) as indicated in figure 6.3 and optimized by the molecular dynamic simulations program GROMACS [89]. For the parallel conformation the simulation leads to diffraction patterns showing at least all reflections found in the experiments, but not for the anti-parallel case, see figure 6.5.

A possibility for anti-parallel structures to overcome the mentioned problems is a shift of 0.167 unit cells along the $z$-axis together with the $y$-shift, see figure 6.4. This modification was proposed by Arnott [91]. It turns out to be a stable configuration after optimization by molecular dynamics simulations, even though the precise values of the shifts differ from the proposed start configuration. Most likely the deviations are due to the fact that molecular dynamic optimization takes place in vacuum. The formation of the $\beta$-sheet crystallites, on the other hand, takes place in the spinning duct in an aquatous solution, under changes of the $p H$ - and a stress-gradiant. So it is likely to assume that the minimal energy structure in vacuum does not necessarily correspond to the minimal structure found in nature. The diffraction patterns of this proposed start conformation, however, agree well with the experiment. 


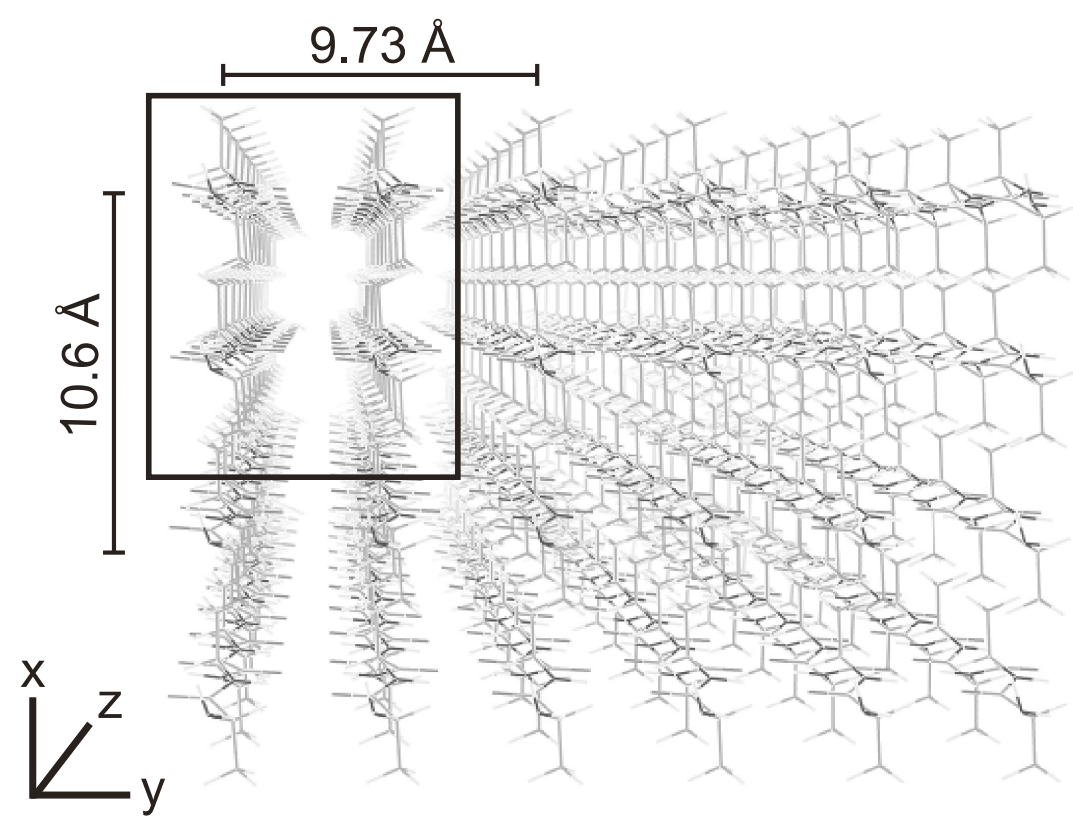

Figure 6.2: Schematic of a $\beta$-sheet obtained by multiplying and rearranging of a protein strands. These structures are also the starting point for modifications of the anti-parallel $\beta$-sheets according to Arnott [91], as shown in figure 6.4. Scattering simulations of structures as shown lack the (120)-peak, regardless of parallel or anti-parallel arrangement. A modification that leads to convenient scattering simulations of parallel $\beta$-sheets is given in figure 6.3. Data by M. H. Meling [84].

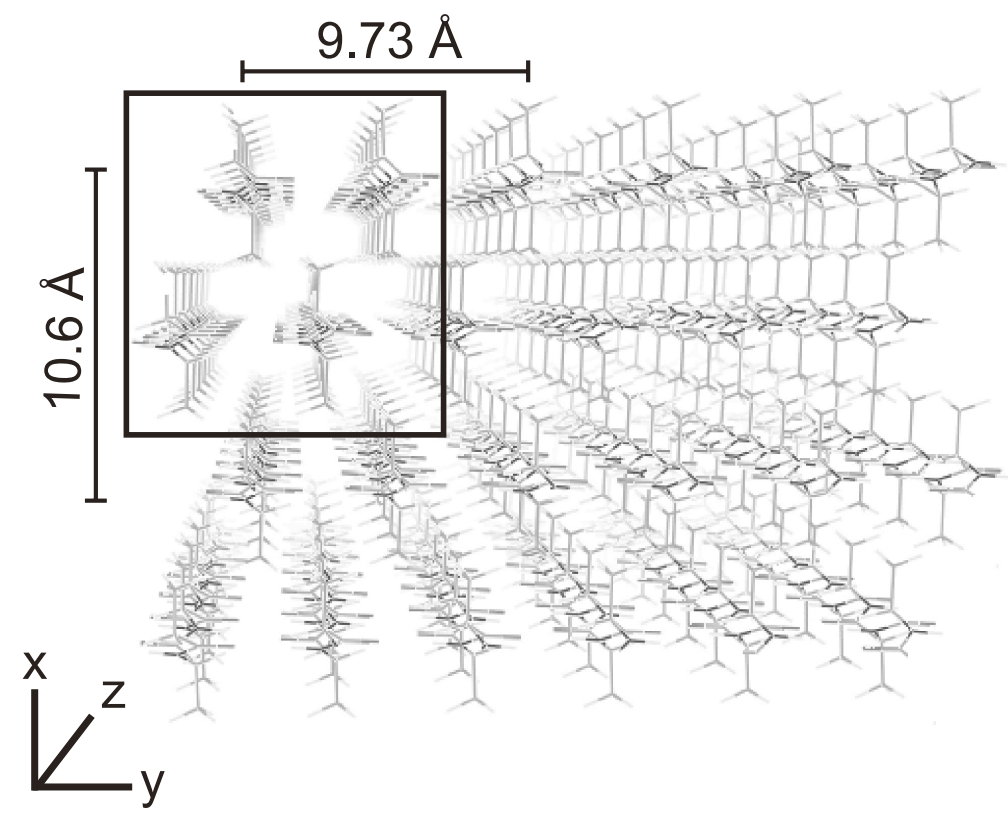

Figure 6.3: Schematic of $\beta$-sheet structures with $y$-shift. The simulation of such modified parallel structures gives all reflections which are found in the experiment. The consistence between scattering simulation and experiment can be improved for parallel structures by molecular dynamic simulations. Anti-parallel structures modified in this way do not give convenient diffraction patterns. They have to be modified as proposed by Arnott [91]. Data by M. H. Meling [84]. 


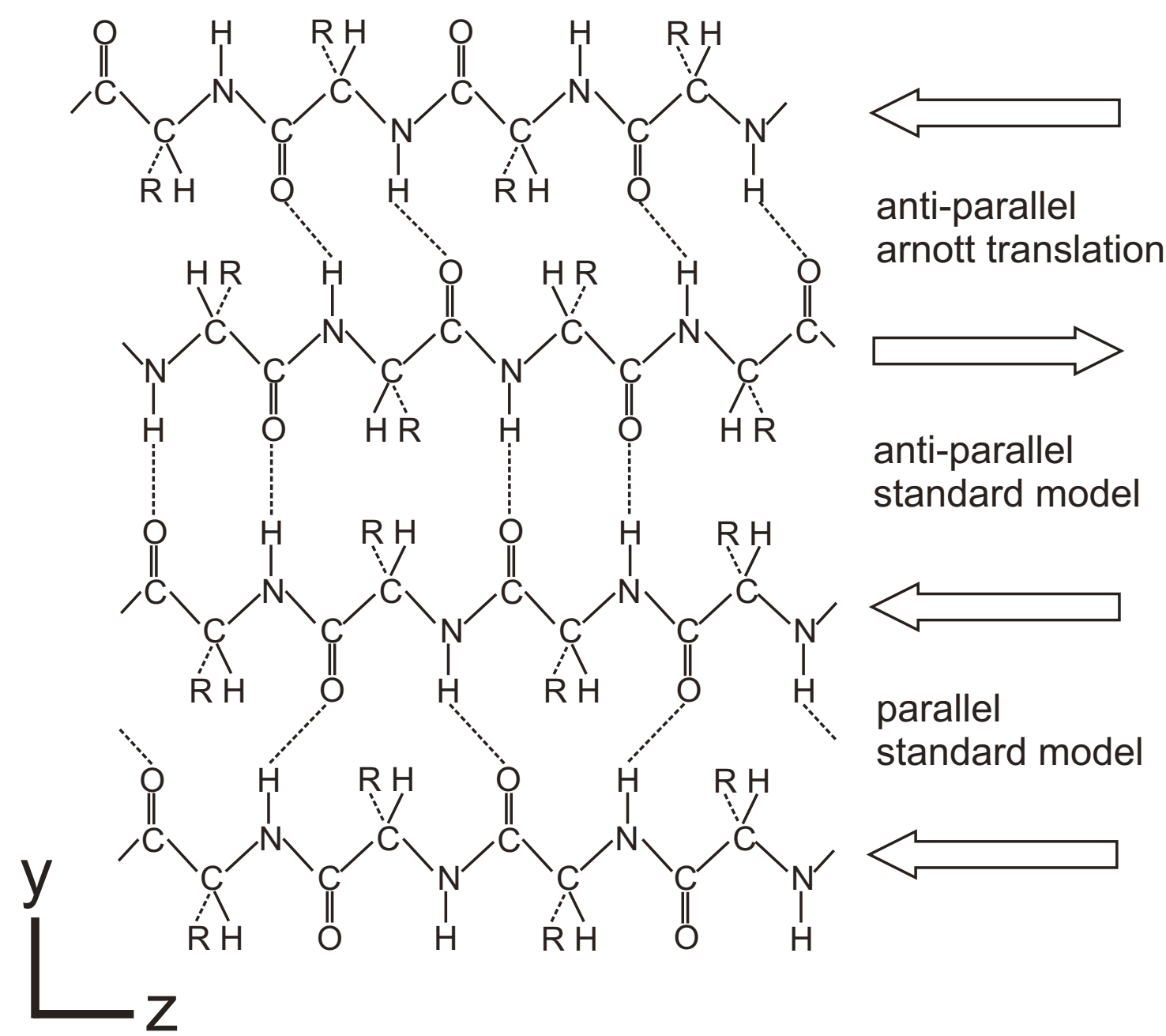

Figure 6.4: Schematic of the modification of an anti-parallel $\beta$-sheet with $y$-shift as proposed by Arnott [91], a standard anti-parallel $\beta$-sheet and a standard parallel $\beta$-sheet. Arnott proposes a displacement of the protein strands in respect to each other by approximately 0.17 lattice constants c or $1.16 \AA$ along the $z$-axis. Scattering simulations of such an 'Arnott' crystallite agree well with the experimentally found diffraction patterns, see figure 6.5 . 


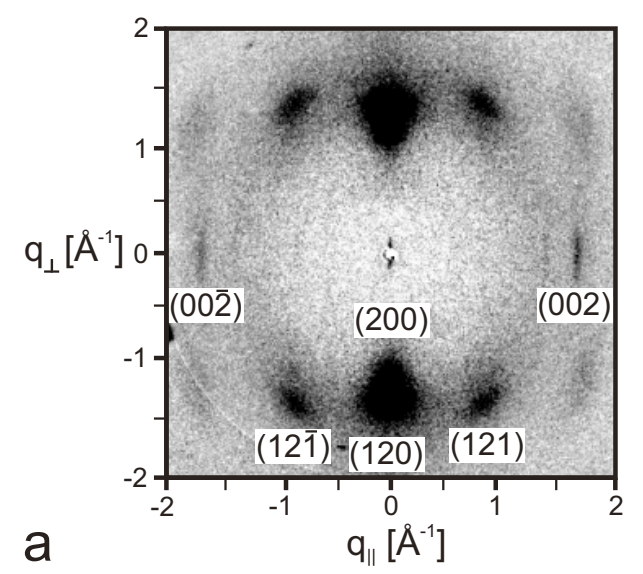

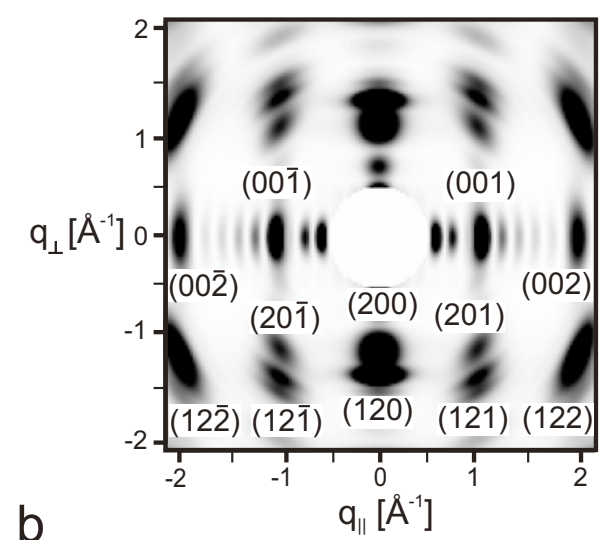

b

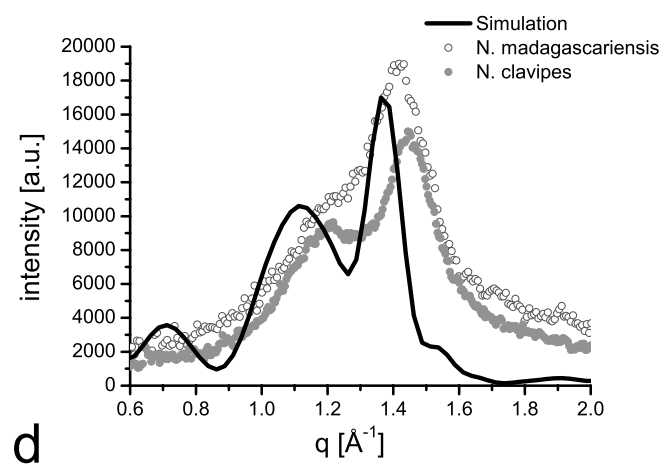

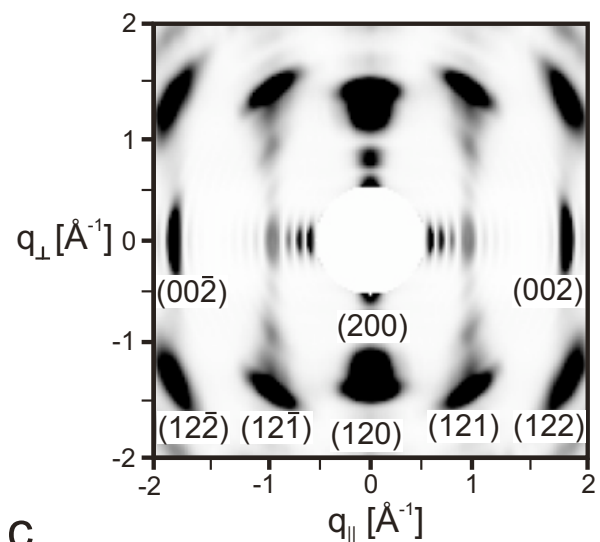

C

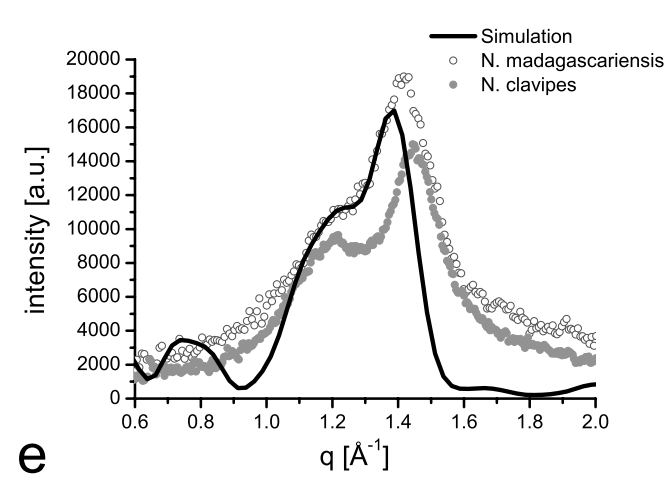

Figure 6.5: (a) Diffraction pattern of a single NM fiber measured at the microfocus beamline ID13, ESRF Grenoble. (b) Simulated diffraction pattern from a parallel crystallite with $y$-shift. Noticeable are (001)- and (201)-reflections which can not be observed experimentally. Data by S. Ulrich. (c) Simulated diffraction pattern from an anti-parallel 'Arnott' crystallite. Data by S. Ulrich. (d) Comparison of the equatorial slices as found experimentally and by scatting simulation of an parallel crystallite with $y$-shift. Peak position and curve shape deviate from the experimental findings. (e) Comparison of the equatorial slices as found experimentally and by scattering simulation of an anti-parallel 'Arnott' crystallite. Peak position and curve shape are quite in good agreement with the experiment. 


\section{Chapter 7}

\section{Thermal Changes in Dragline Fiber Bundles}

In this chapter we present an investigation of the structure of spider silk $\beta$-sheet crystallites by $X$-ray diffraction over a broad temperature range between ambient temperatures and thermal denaturation conditions. The intensity in the equatorial plane was recorded, as described in chapter 3.2.1. Accordingly the intensity profiles are displayed versus the scattering angle $2 \theta$.

We find that the $\beta$-sheet crystallites are extremely stable against thermal denaturation. Spider silk is mostly studied in a temperature range from $10^{\circ} \mathrm{C}$ to $60^{\circ} \mathrm{C}$, corresponding to typical temperatures of the spider's habitats. Calorimetry studies have been reported at higher temperatures, and give some evidence for a glass transition at $150-160^{\circ} \mathrm{C}[22 ; 92]$. Here we study the spider silk structure by fiber diffraction over a wide temperature range up to thermal denaturation and focus on the crystalline fraction is easily accessible by X-ray diffraction.

\subsection{Samples, Measurement and Data Analysis}

Dragline fibers of the three species named in chapter 4 of the genus Nephila have been studied. The fibers were collected as described in the same chapter and reeled directly onto the sample holder.

For the temperature measurements a sample holder was used suitable to expose three different samples subsequently at one temperature. For temperature control we used a home built heating device consisting of an aluminium block $(5 \mathrm{~cm} \times 4 \mathrm{~cm} \times 1.5 \mathrm{~cm})$, a $50 \mathrm{~cm}$ heating wire (Thermocoax, France) and a thyristor based control unit. The heating wire was partly embedded in the aluminium block and partly wrapped around it. The temperature $T$ was controlled with a Pt100 temperature sensor. The scattering intensity was measured over an angular range between $2 \theta=6^{\circ}$ to $2 \theta=40^{\circ}$, scanned with a step size of $0.1^{\circ}$. The exposure time varied between the different silk samples from 60sec/step to $90 \mathrm{sec} / \mathrm{step}$ depending on the scattering signal's strength. First, all samples were measured at room temperature $\left(25^{\circ} \mathrm{C}\right)$. The next temperature point was $50^{\circ} \mathrm{C}$. From $T=50^{\circ} \mathrm{C}$ on, $T$ was increased in steps of ten degrees up to $250^{\circ} \mathrm{C}$. The fibers were orientated vertical and the scans were taken perpendicular to the fibers' axes. In this 
range, the (120)- and the (200)-peak dominate the scattering distribution, see figure 7.1. For comparison, ex-situ annealing measurements were carried out on one sample of Nephila clavipes dragline to investigate irreversible changes by temperature. To this end, the sample was first kept for $30 \mathrm{~min}$ in a preheated oven and then measured at room temperature. Subsequently the same sample was heated up to the next temperature in the same way and measured again. Each intensity profile was treated as follows: A selected range around the two main peaks in between $12.5-25^{\circ}$ was fitted with two Lorentzians (Origin 7.0, MicroCal), see figure 7.1. The peak position in $2 \theta$, fwhm, intensity $(I)$ and the area under the peak $(A)$, also denoted as integrated peak intensity, were determined for the two respective peaks, and used for further analysis. From the fwhm values, the average crystallite sizes $L_{200}$ and $L_{120}$ were calculated using the Debye-Scherrer formula (equation 3.17, page 28). To compare the results for different $T$, and for each reflection and each spider species, the intensities and the other peak parameters were normalized to the values measured at $25^{\circ} \mathrm{C}$, the starting point of the temperature scan. For example, the intensity of the (120)-reflection was normalized as

$$
I_{(120)}^{N S}(T)=\underbrace{I_{(120)}^{N S}(T)}_{\text {rawdata }} / I_{(120)}^{N S}\left(25^{\circ} \mathrm{C}\right) .
$$

After inspection of the results, we found that samples of the three species behave quite similar, and the average of the three different Nephila species was considered in the analysis, e.g. for the intensity $I_{120}(T)=\left(I_{120}^{N S}(T)+I_{120}^{N C}(T)+I_{120}^{N M}(T)\right) / 3$. The standard deviation estimated from the three values were used for the error bars in the $T$ plots.

\subsection{Results}

In figure 7.2a, scattering profiles are shown for five different temperatures to illustrate the evolution with $T$. Between $25^{\circ} \mathrm{C}$ and $150^{\circ} \mathrm{C}$ there is nearly no change in the scattering profiles. Beginning at $150{ }^{\circ} \mathrm{C}$ the peak intensity is decreasing and the peaks are sharpening at the same time, i.e. the fwhm is decreasing. However, there is a notable difference between the (120)- and (200)-peaks. Initially, the (120)-reflection is more intense than the (200)-reflection but with increasing $T$, the (120)-reflection decreases faster than the (200)-reflection. Peaks are observable up to about $250^{\circ} \mathrm{C}$. The decrease of the fwhm saturates at about $194^{\circ} \mathrm{C}$, from where on the width is found to increase again. While the thermal denaturation pathway seems to be broad and complicated, one can say that denaturation starts at approximately $200^{\circ} \mathrm{C}$, which is extremely stable for a protein. During heating and especially at temperatures above $150{ }^{\circ} \mathrm{C}$ the samples start to contract, see figure 7.3. Therefore, beside the thermal effects internal stress of the fibers may occur, if a relaxation is hindered by the sample holder. Figure 7.3 shows the contraction measured with a telescope zoomed to measure the length of a single fiber placed in a temperature-controlled oven. The maximum contraction goes up to $20 \%$ of its original length. Thermal contraction is typically associated with entropic elasticity. In the present case irreversible changes and the loss of water content are probably the primary cause for the effect at high $T$. 


\subsubsection{Lattice constants}

From the $2 \theta$-peak positions the lattice constants $a$ and $b$ of the Bragg planes were calculated Bragg's formula (equation 3.15, page 28) and the relation between $d$ spacing and lattice constants for an orthorhombic lattice given by equation 3.16. It was found that the $d$-spacing corresponding to the (120)-peak increases from $4.41 \AA$ at $25^{\circ} \mathrm{C}$ up to $4.44 \AA(\approx 0.7 \%)$ at $143^{\circ} \mathrm{C}$, and then decreases again to $4.42 \AA$, see figure 7.4a. For the (200)-peak, the respective $d$-spacing shows nearly the same behavior. It increases by $1.1 \%$ of its initial value of $5.36 \AA$ then decreases down to $5.39 \AA$. However, above $200^{\circ} \mathrm{C}$ it increases again up to $5.44 \AA$, see figure 7.4 a. With the two $d$-spacings, the changes of the lattice constants $a$ and $b$ were calculated from equation 3.16. Correspondingly, the lattice constant $a$ changes by $1.12 \%$ and $b$ by $0.41 \%$ from $25^{\circ} \mathrm{C}$ to $143^{\circ} \mathrm{C}$, see table 7.1 . Therefore the temperature expansion coefficient is highly anisotropic along the two respective lattice constants. The thermal expansion varies by a factor of three in the two respective directions. This is interesting because the (100) direction associated with the lattice constant $a$ corresponds to the non-covalent bonds between the amino acid side groups of neighboring $\beta$-sheet strands. Contrarily, the direction along $b$ which is found to be more stable with temperature, is associated with hydrogen bonds between different $\beta$-sheet strands. It seems quite plausible, that the hydrogen-bonds exhibit higher thermal stability and less extensibility than the non-covalent bonds between the amino acid residues. Both coefficients are significantly higher than those of typical solids. For comparison, we note that the expansion coefficient of the interlayer spacing of a graphite single crystal between $78 \mathrm{~K}$ and $297 \mathrm{~K}$ is about $7.43 \cdot 10^{-5}$ [93].

The comparison with the ex-situ determination of the lattice constant is interesting, since all reversible thermal expansion effects should obviously not appear after cooling the sample down again. The residual irreversible changes appearing in particular at medium and higher temperature indicate a decrease of the $d$-spacing, see figure $7.4 \mathrm{~b}$. An approximately linear decrease of $-4.27 \cdot 10^{-4} \AA / \mathrm{K}$ is observed.

Table 7.1: Lattice constants $a$ and $b$ of the orthorhombic $\beta$-sheet crystallite at two different temperatures., as calculated from equation 3.16. Exp. coeff. = Expansion coefficient.

\begin{tabular}{lrrrr} 
& $25^{\circ} \mathrm{C}$ & $143^{\circ} \mathrm{C}$ & Exp. coeff. $[\AA / \mathrm{K}]$ & change \\
\hline $\mathrm{a}[\AA]$ & 10.72 & 10.84 & $1.017 \cdot 10^{-3}$ & $1.12 \%$ \\
$\mathrm{~b}[\AA]$ & 9.68 & 9.72 & $3.39 \cdot 10^{-4}$ & $0.41 \%$ \\
\hline
\end{tabular}

\subsubsection{Peak Intensity I(T) and Peak Area A(T)}

Figure 7.5 shows the thermal characteristics of the maximum peak intensity $I(T)$ and the peak area $A(T)$ (integrated intensity) of both the (120)- and the (200)-peak. While $A_{120}(T)$ and $I_{120}(T)$ are both steady or monotonously decreasing, $A_{200}(T)$ 
and $I_{200}(T)$ both show a maximum around $194{ }^{\circ} \mathrm{C}$. Peak intensity and area are both strongly related to the number of crystallites $N$ in the scattering volume. Let us first interpret $I(T)$ and $A(T)$ under the simplified assumption that the intensity changes are solely determined by the $T$-dependence of the structure factor, while the form factor or equivalently the structure of the unit cell remains constant. Secondly, we note that there is orientational, but no positional long-range order of the crystallites, so that the results can be understood as the average of single crystallite scattering. It follows from elemental kinematic scattering theory [94] that

$$
\begin{aligned}
I & =c_{I} N L^{2} \\
A & =\int \frac{I}{4 L^{2}\left(q-q_{0}\right)^{2}+1} d q=c_{A} N L,
\end{aligned}
$$

where $L$ is the size of the crystal along the corresponding lattice direction. $c_{I}$ and $c_{A}$ are proportionality coefficients. The number of scatterers and the size of the crystal may change, as long as the form factor and all other quantities are constant $c_{I}$ and $c_{A}$ are fixed which we assume here. For the sake of simplicity we also restrict our consideration to the case of Lorentzian lineshapes, which was observed experimentally. In principle these two equations can be used to determine the unknowns $N$ and $L$. However, $c_{I}$ and $c_{A}$ are unknown, since the form factor is unknown, and the intensity values are not in absolute values. Hence we can use the reference temperature of $25^{\circ} \mathrm{C}$, to get $c_{I}=I\left(25^{\circ} \mathrm{C}\right) /\left(N\left(25^{\circ} \mathrm{C}\right) L\left(25^{\circ} \mathrm{C}\right)^{2}\right)$, and $c_{A}=I\left(25^{\circ} \mathrm{C}\right) /\left(N\left(25^{\circ} \mathrm{C}\right) L\left(25^{\circ} \mathrm{C}\right)\right)$. Accordingly, we get $N(T) / N\left(25^{\circ} \mathrm{C}\right)$ and $L(T) / L\left(25^{\circ} \mathrm{C}\right)$ normalized to the reference state. Of course, most directly and without knowledge of proportionality constants, the average crystallite size along the (hkl) direction can be obtained from the fwhm, see further below. Figure 7.6 shows the $N(T) / N\left(25^{\circ} \mathrm{C}\right)$ curves for the two peaks. Beginning at $100^{\circ} \mathrm{C}$ both curves decrease. However, the number $N$ determined by the above procedure has to be identical within the experimental errors to be consistent. We see that the experimental results for $I(T)$ and $A(T)$ cannot be simply understood from changes of the structure factor, but that changes in the unit cell structure must occur. Nevertheless we assume that the trend of a decreasing number of crystallites is correct. A simple experimental argument that the $T$ dependence cannot be explained based on the structure factor alone is the changing intensity ratio $I_{200} / I_{120}$. From figure $7.5 a$ we can see that between $150^{\circ} \mathrm{C}$ and $200{ }^{\circ} \mathrm{C}$, the (200)peak becomes stronger and the ratio $I_{200} / I_{120}$ increases with $T$, see figure 7.2. Thus the (200)-peak increases in comparison to the (120)-peak. On the other hand the position of the peaks stays constant. Therefore we can assume that the crystal structure of the crystallites remains the same. We conclude that changes in the form factor rather than the structure factor are responsible for $I_{200} / I_{120}$, e.g. a change in the crystallite's amino acid composition. Such an effect should be similar to the different scattering intensities of Nephila and Bombyx mori silk, see figure 7.1. For BM the intensity of the (120)- and (200)-peak are nearly the same. Chemically, the main difference between the $\beta$-sheet crystallites of $\mathbf{B M}$ and spider silk is attributed to the composition. While BM crystallites consist of approximately half alanine and half glycine [41], the $\beta$-sheets of Nephila are believed to consist mainly of alanine. 


\subsubsection{Size of the Crystallites}

Next we consider the crystallite size $L$, which was directly computed from the fwhm by the Debye-Scherrer formula (equation 3.17, page 28) with a shape factor $k=0.9$. As is well known from equation 3.17 the peak's radial width (fwhm) is inverse to the crystallite size. Therefore the decrease of the fwhm, which starts at $150^{\circ} \mathrm{C}$, indicates an increase of average crystallite size, see figure 7.7. From the intensity decrease (see section 7.2.2) we know that the number of crystallites decreases with $T$. One may wonder whether the apparent crystallite growth occuring along with a decrease in crystallite number density can be explained by a preferred 'melting' of small crystallites. Unfortunately, direct access to the size distribution of the crystallites is not possible. Note that the size distribution influences the lineshape, but the experimental accuracy is not good enough to exploit this. Therefore, we test the idea of 'crystallite growth by selective removal of small crystallites' by means of a simple estimate. For that purpose we switch from crystallite size $L$ to number of Bragg planes $M$. In an orthorhombic crystal system $M$ is related to $L$ by $L_{h k l}=M_{h k l} \cdot d_{h k l}$ with $d=\left(h^{2} / a^{2}+k^{2} / b^{2}+l^{2} / c^{2}\right)^{-0.5}$. For all three spider species $M_{120} \approx 11$ and $M_{200} \approx 9$, so that in the following we use an average value of $M=10$. For illustration, let us compare the results of the measurement at $194^{\circ} \mathrm{C}$ with our estimation. $L_{120}\left(194^{\circ} \mathrm{C}\right)$ is 1.6 times larger than the $25^{\circ} \mathrm{C}$ value, while the ratio in number density is $N_{120}\left(194^{\circ} \mathrm{C}\right) / N_{120}\left(25^{\circ} \mathrm{C}\right)=0.32$. So on the one hand, the average number of Bragg planes has to increase from $M=10$ to $M=16$, on the other hand the total number of crystallites has to decrease to roughly one third of the initial crystallites. Assuming the extreme case of equipartition, where all numbers of Bragg planes between 1 and 19 are equally likely to occur, all crystallites with $M<14$ have to melt to raise the average accordingly. Only crystallites with 14 to 19 Bragg planes remain. This numerical example shows that melting of small crystals alone can hardly be a realistic cause for the observed trend. Note also that the assumption of equipartition is very unrealistic in view of the highly repetitive structure of Spidroin 1 and Spidroin 2 [31; 32], compare also appendix B, as well as in view of crystallite growth and stability. More realistic size distributions would accentuate the trend. Thus real growth of crystallites at higher temperatures must be the cause of the observed effect. Higher temperatures may more easily allow for the activation energy associated with crystallization of additional chain segments with a more disordered sequence [95]. The $T$ dependent results were corroborated by ex-situ temperature measurements on NC. As a matter of fact one can see the same effects although the curves are slightly shifted to higher temperatures, see figure 7.7. The maximum crystallite size occurs at $220^{\circ} \mathrm{C}$, compared to about $200^{\circ} \mathrm{C}$ for the in-situ measurements. Denaturing seems to occur at higher temperatures, indicating that 30 minutes annealing time is not long enough due to kinetic effects in thermal denaturing. However, these temperatures are distinctly smaller than the denaturation temperatures given in [96] for Nephila pilipes, a species from the Asia-Pacific region. 


\subsection{Summary and Discussion}

Our measurements indicate a surprisingly high thermal stability of spider silk against denaturing. At the same time, we found evidence for a structural change of the $\beta$-sheets with increasing $T$, intermediate between ambient and denaturing temperatures. Two possible explanations for the increase in crystallite size with $T$ have been discussed:

(i) There is no real growth. The observed 'growth' is observed because small crystallites break up (melt) earlier than bigger crystallites.

(ii) There is a real growth of the crystallites, anisotropic in the two azimuthal lattice directions. However, this growth cannot outnumber the melting of some crystallites, so that the scattering intensity decreases while the peak sharpens. The growth of the crystallites is accompanied by a change in composition explaining the change in the intensity ratio of the two strongest peaks.

The quantitative changes in the diffraction pattern indicate that the second explanation is more likely. They also show that not only the crystal size (structure factor), but also its composition (form factor) must change. A change in the amino acid composition during heating could also lead to additional peaks which are not observed for a $\beta$-sheet crystallites dominated by alanine. While the observed peaks are dominating the diffraction pattern, further experiments using synchrotron radiation and area detectors could shed more light on the $T$ dependent structural changes in spider silks. 


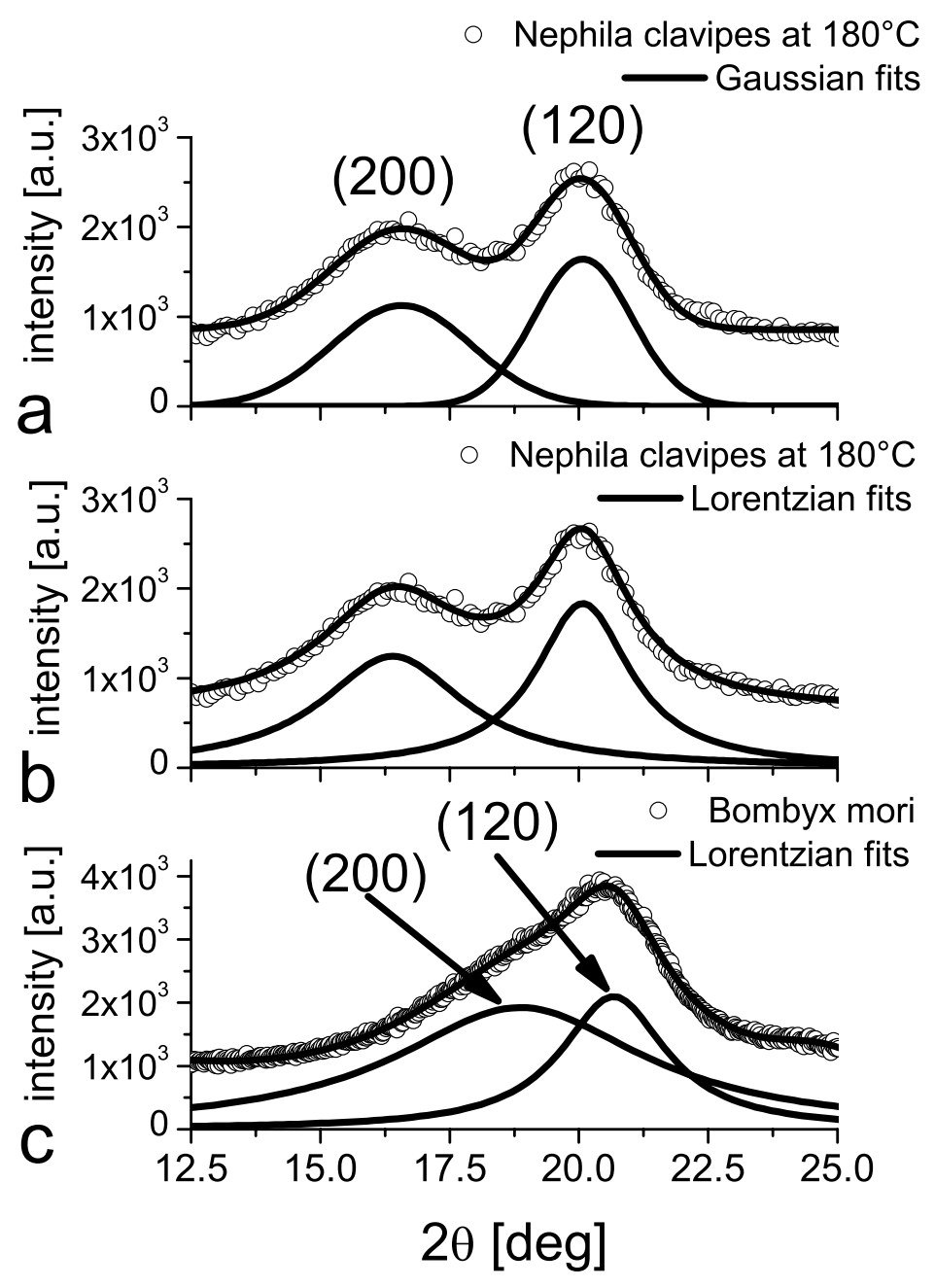

Figure 7.1: Examples for the scattering profiles showing the characteristic (200)- and (120)-peaks of the quasi-orthorhombic lattices for $a$ and $b$ Nephila clavipes, and $c$ B. mori. $(a, b)$ Intensity profile corresponding to the intensity along the equatorial plane, see figure 3.10a, crossing the two main azimuthal reflections. (a) The profiles are fitted by a double Gaussian curve. Note the deviation between the measured curve and Gaussian curve around $2 \theta=22.5$. (b) The profiles are fitted by a double Lorentzian curve which describe the profile more exact than Gaussians. For spider silk, the (200) intensity is typically weak at room temperature but increases with $T$. (c) Intensity profile of a silk sample from the silkworm B. mori fitted by two Lorentzians. The intensities of the two equatorial peaks is nearly the same. 

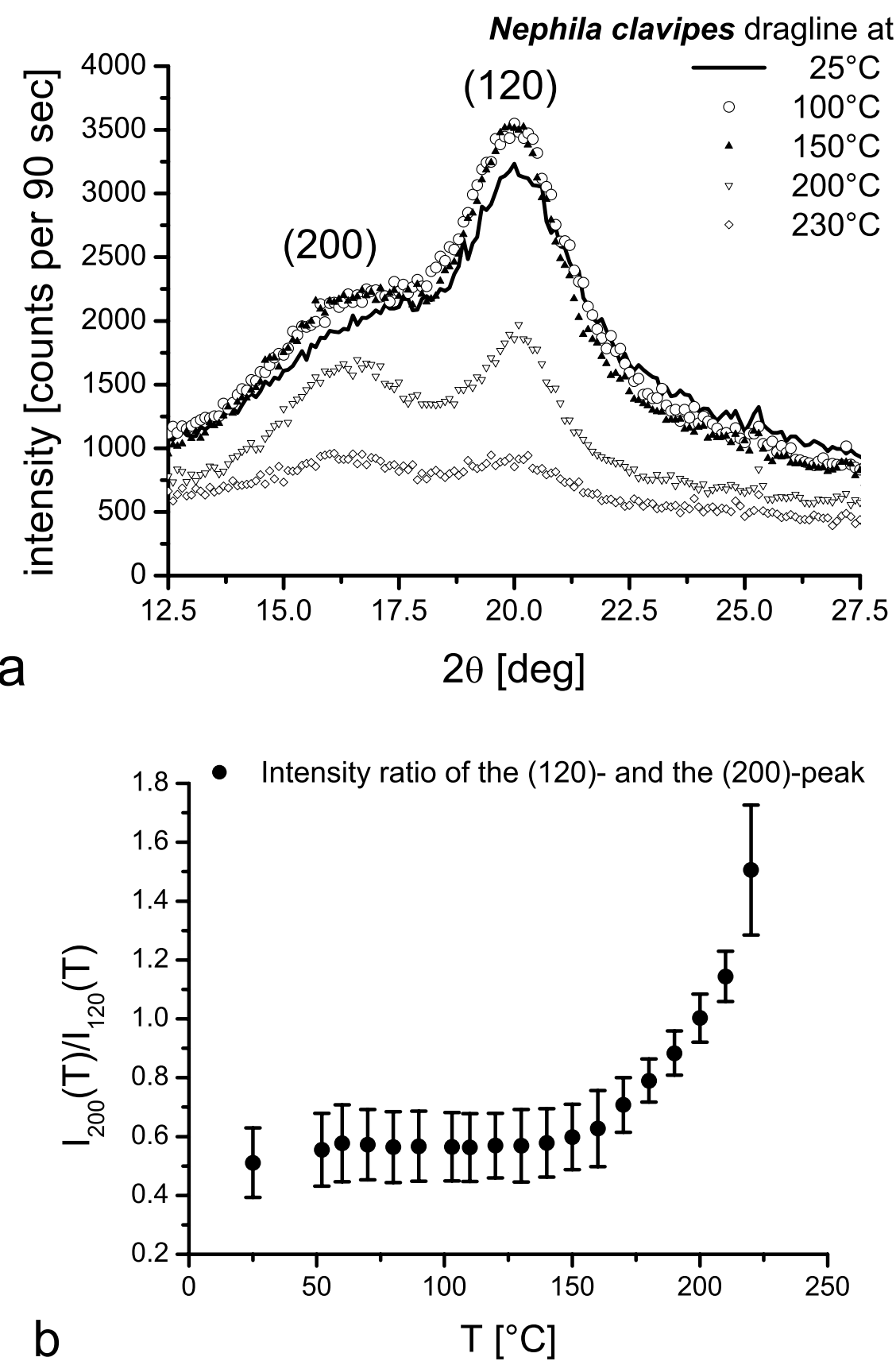

Figure 7.2: (a) Intensity profile of a NC fiber bundle at five different temperatures. (b) The intensity ratio of the (120)- and (200)-peak versus temperature. The (200)-peak is found to increase in comparison to the (120)-peak. 


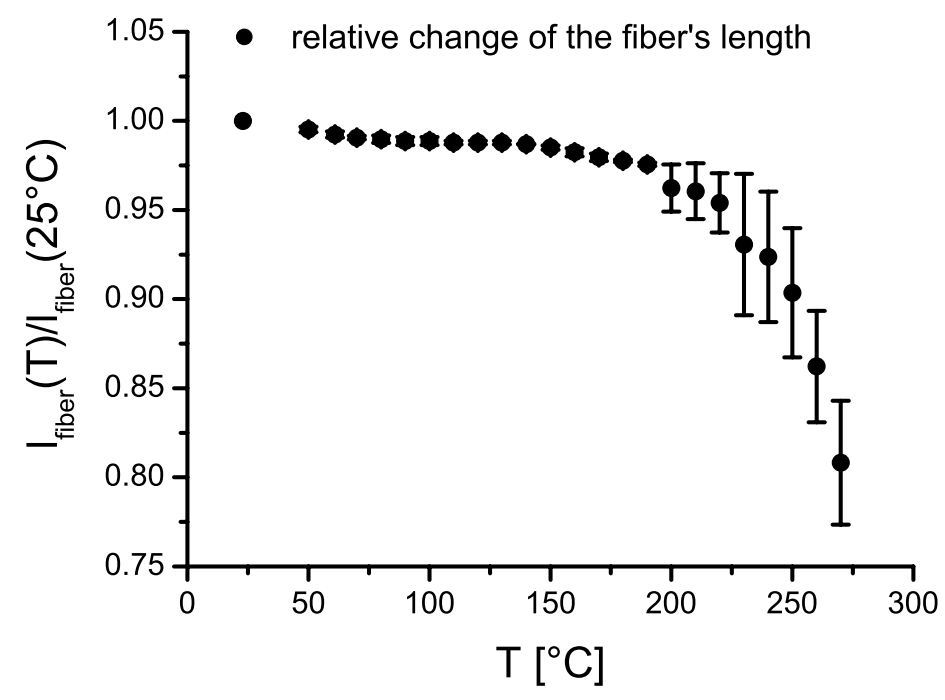

Figure 7.3: Relative change of length, observed for NM dragline. 

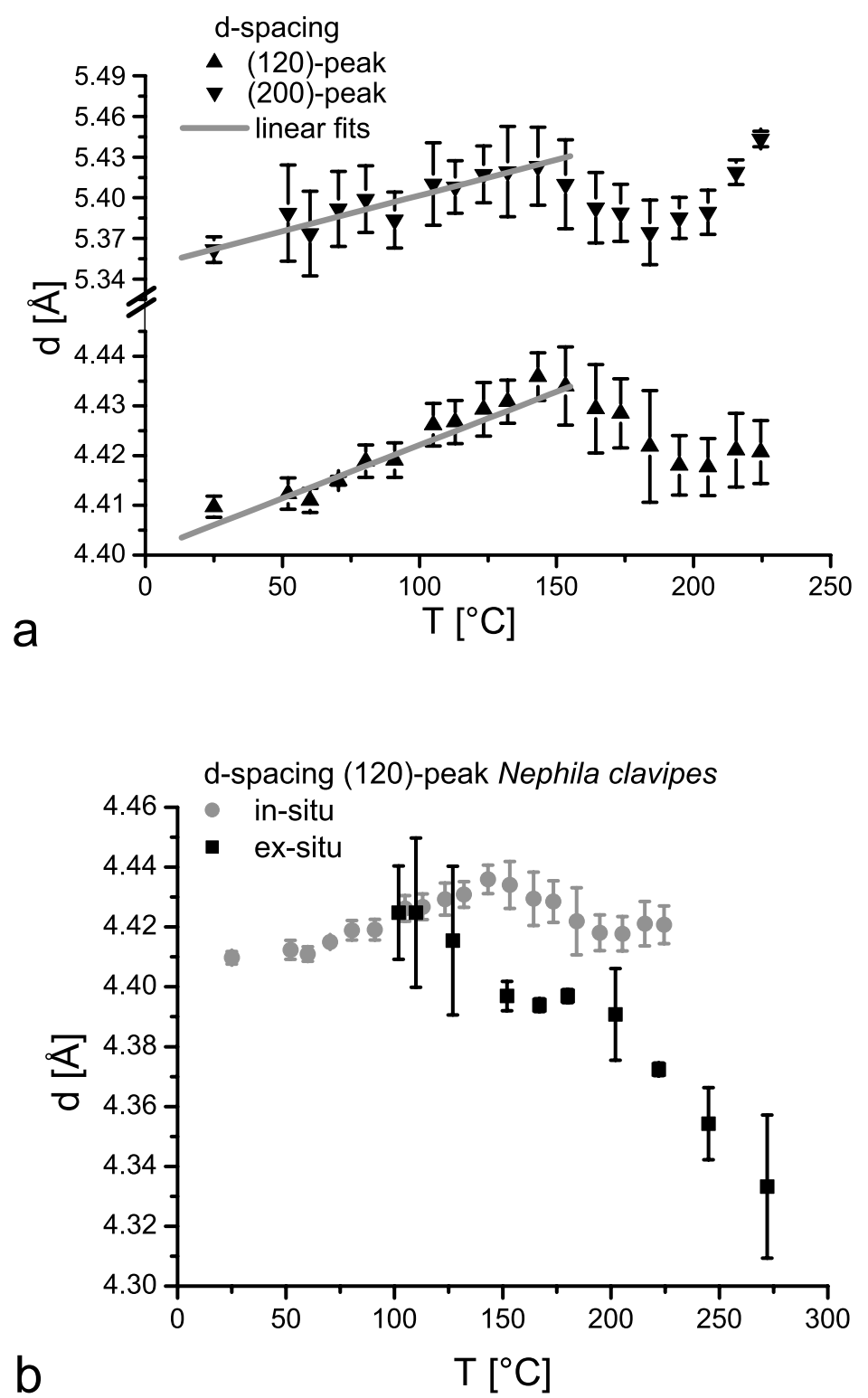

Figure 7.4: (a) Distance of the Bragg planes (d-spacing) obtained from the (120)- and (200)peak position, averaged over the Nephila species. The initial linear increase gives the thermal expansion coefficient along the two respective lattice directions. At higher $T$ irreversible changes are observed. (b) Comparison between the in-situ temperature measurements and the ex-situ measurements after annealing (NC (120)-peak). During in-situ temperature experiments the dspacing undergoes a maximum, while it constantly decreases during ex-situ experiments. 


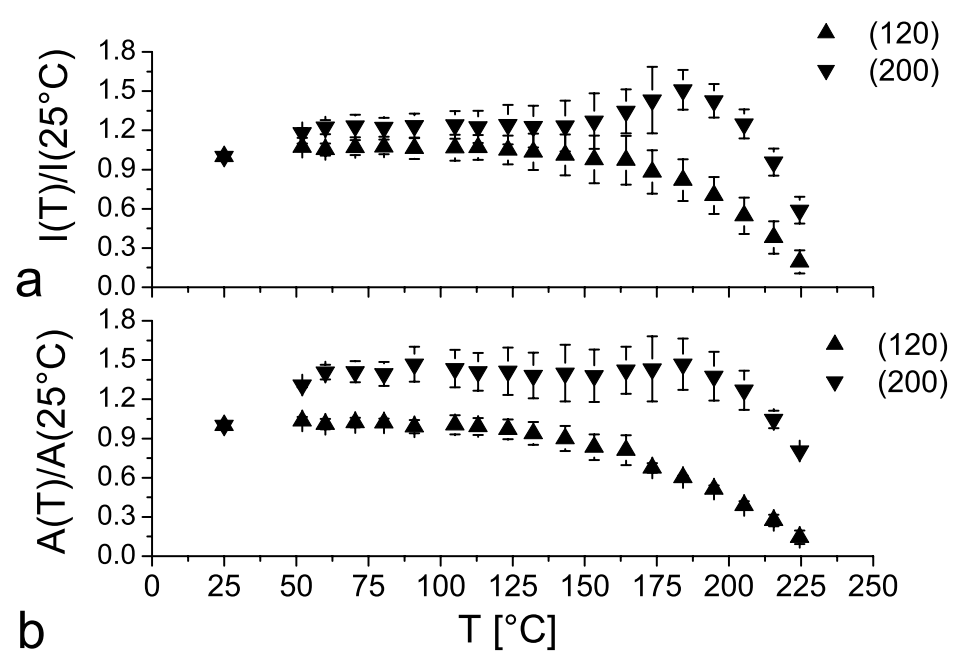

Figure 7.5: (a) Normalized peak intensity (I) of the (120)-peak and the (200)-peak vs. temperature. (b) Integrated intensity (area $A(T)$ ) of the (120)-peak and (200)-peak, again normalized by the result at $\mathrm{T}=25^{\circ} \mathrm{C}$.

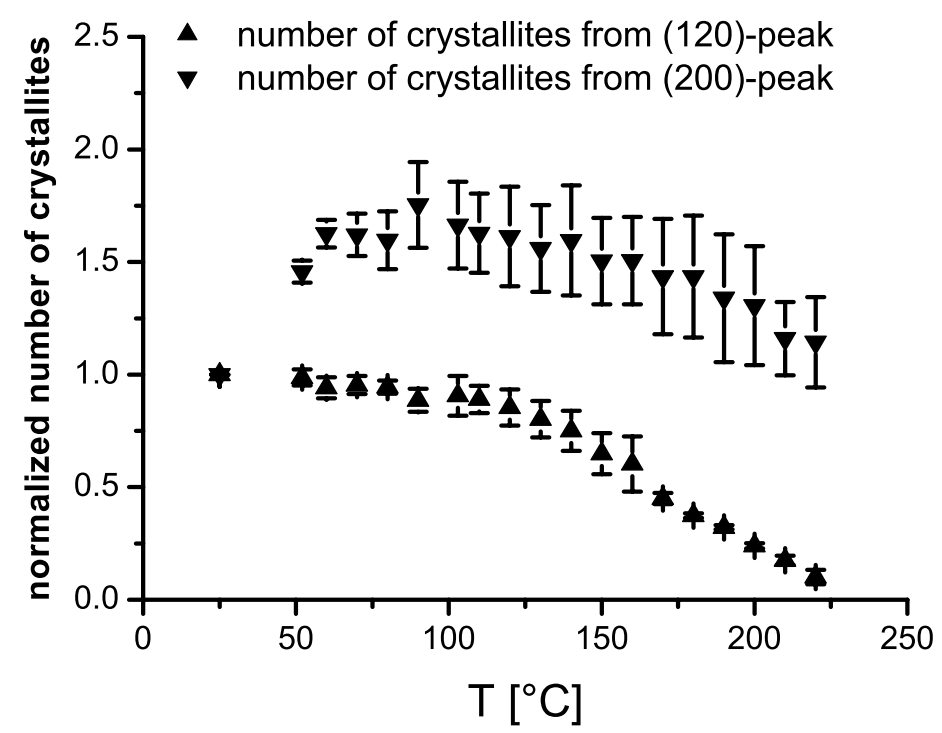

Figure 7.6: Normalized number of crystallites $N(T)$ in the scattering volume compared to room temperature. From both peaks the simplified analysis shows a decrease at high $T$. The result are inconsistent, in that $N(T)$ is different for the two curves. This falsifies the assumption that the observed intensity changes are solely based on the structure factor. 


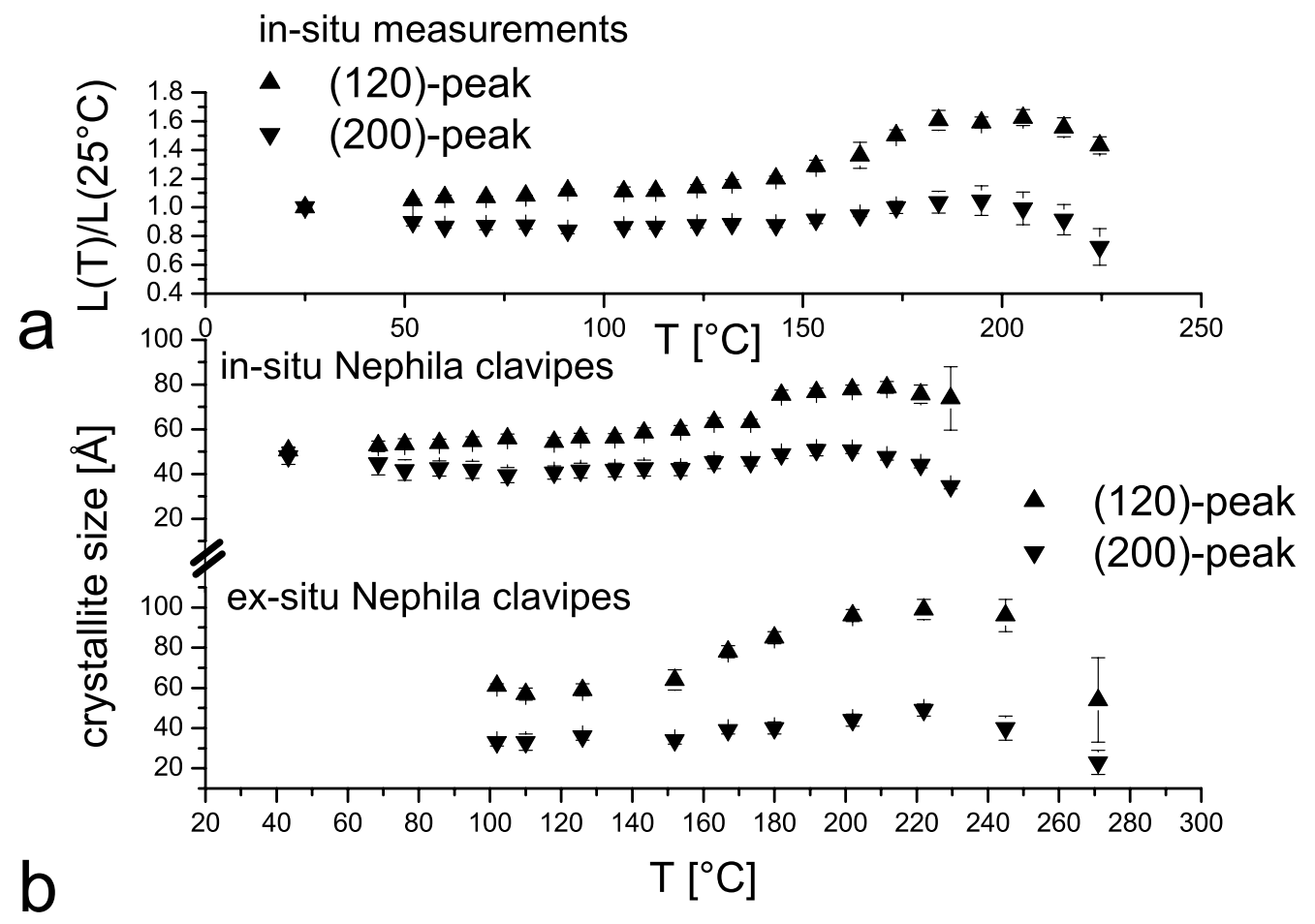

Figure 7.7: (a) Normalized size of the crystallite $L(T) / L\left(25^{\circ} \mathrm{C}\right)$ vs. temperature for the three species ensemble calculated from Lorentzian fits of (120)-peak are shown. The changes of size calculated from the (120) peak is much stronger than the one of (200).

(b) Comparison between in-situ temperature measurement and ex-situ annealing of NC dragline. 


\section{Chapter 8}

\section{Single Fiber Diffraction - The Influence of Strain}

In order to study changes of the molecular structure under external strain we have combined single fiber X-ray diffraction experiments with in-situ force-strain measurements. Essential for such studies is the use of single fibers otherwise it cannot be assured that the strain is uniform in the entire scattering volume. The measurements were performed at the ESRF's microfocus beamline ID13 in Grenoble as described in chapter 3.3.1. The fibers were mounted in the LEX 810 High Resolution Extensometer (Dia-Stron Ltd., Andover, Hampshire, UK), described in chapter 3.5. The samples were approximately $30 \mathrm{~mm}$ long and pulled with a speed of $0.01 \mathrm{~mm} / \mathrm{min}$ up to break. The experiments took place at the beamline's standard conditions at $50 \% \mathrm{RH}$ and $23^{\circ} \mathrm{C}$ which were stable enough to refrain from other setups for environmental control. Humidity and temperature were observed during the experiments with a testo 608-H1 hygrometer (Testo AG, Lenzkirch, Germany). The focusing with a pair of short focal length Kirkpatrick Baez (KB) mirrors [97] of the $12.7 \mathrm{keV}$ X-ray beam to a $7 \mu \mathrm{m}$ spot at the sample provided a sufficient flux density $\left(6.8 \cdot 10^{15} \mathrm{cps} / \mathrm{mm}^{2}\right)$ to obtain diffraction patterns from single dragline fibers, but also increases the risk of radiation damage. To prevent artifacts from radiation damage, we have performed the experiment in the following way:

(i) Elongation of the sample to a preset strain

(ii) Keeping the sample at this strain

(iii) Taking one $1 \mathrm{sec}$ exposure

(iv) Translation of the sample by $20 \mu \mathrm{m}$ to the left along the horizonally positioned fiber

(v) Realignment of the sample in the beam

(vi) Ten repetitions of step (iii) and (iv) to obtain eleven $1 \mathrm{sec}$ exposures at the preset strain value

(vii) Reiteration of steps (i)-(vi) at the next higher strain value. 
The strain-pauses lasted up to 5min. During every pause, relaxation occurs in the fiber, see chapter 5. The pauses were necessary to keep the fiber stable in the beam, which is essential, given the $7 \mu \mathrm{m}$ spot and $5 \mu \mathrm{m}$ fiber. Therefore the force-strain curves exhibit a sawtooth pattern, see figure 8.1a. The relaxation of the fibers measured at ID 13 was compared with the relaxation of non irradiated fibers, and no differences were found.

\subsection{Results}

The two-dimensional (2D) intensity distribution was analysed in the form of different one-dimensional (1D) cuts or slices, compare chapter 3.4. The lateral width of the $1 \mathrm{D}$ slices was optimized for signal-to-noise. At a pixel size of $157.88 \mu \mathrm{m}$ corresponding to $\Delta q \simeq 7.2 \cdot 10^{-3} \AA^{-1}$, typically eleven one-pixel slices were summed up to generate a 1D slice. From the equatorial slices through the beam center and the equatorial peaks, the peak positions $q_{120}$ and $q_{200}$ of the two strongest peaks and their fwhm were determined by least-square fitting, see chapter 3.4 and figure 3.10. The same procedure was applied to the (002)-peak in the meridional plane. As is well known, the peak positions $q_{h k l}$ gives information about the lattice constants of the pseudo orthorhombic unit cell of the $\beta$-sheet crystallites [42; 41], while the fwhm is related to the crystallite size according to the Debye-Scherrer formula. Both the vertical slice through the (002)-peak as well as the off-axis slice through the (120)- and (121)-peaks contain information about the lattice parameter $c$. The intensity ratio of the (120)- and (200)-peaks varies between the three Nephila species, but the (200) intensity is always significantly smaller than (120). Therefore the fitting errors of the (200)-parameters are always considerably higher than those of (120).

Starting with the zero strain reference state, it was found that $q_{120}$ and $q_{200}$ values for all samples measured where somewhat smaller than the $q$-values expected from the work of Warwicker [42], which is a classical reference for fiber proteins. However, the deviations are within the bounds of the Warwicker 3 class. Only for the species NM the diffraction was intense enough to enable a full analysis of the (002)-peak, and the $q_{002}$ values obtained from this species are nearly in perfect agreement with literature. We now discuss the structural parameters and the associated changes of the diffraction pattern in the following sequence: 1.) angular width of the refections indicative for orientational distribution of the crystallites, 2.) radial width of the reflections indicative of the crystallite size, and finally 3. ) the position of the peaks reflecting the lattice constants. All parameters, including those which are not represented graphically, are tabulated in table 8.1. The error bars indicate the standard error of mean $=$ standard deviation $/ \sqrt{n}$, were $n$ is the number of independent measurements for each strain value $\varepsilon$.

\subsubsection{Angular Width and Crystallite Orientation}

From arc slices through the (120)-peak, an angular distribution $a w_{120}$ of the intensity is obtained. This intensity distribution reflects the distribution of the tilt angles $\phi$ of the local $\beta$-sheet $z$-axis with the fiber axis. The crystallites are always 
aligned with their longest side ( $z$-axis) parallel to the direction of the fiber. From $a w_{120}$ an orientational order parameter $o p=\left(3 \cos (0.425 a w)^{2}-1\right) / 2$ can be defined $[5 ; 12], a w_{120}(\varepsilon)$ is analysed by a linear regression. For $\mathbf{N C} a w_{120}$ decreases clearly (figure 8.2a), less significantly for NS (figure 8.8) and within the experimental errors, it is constant for NM, see also figure 8.2a and table 8.1.

\subsubsection{Radial Width and Crystallite Size}

From the radial width (fwhm) of the reflection $\Delta q_{h k l}$ measured along the equatorial and meridional slices, the crystallite size $L_{h k l}$ was calculated from DebyeScherrer formula $L=k \cdot 4 \pi / \Delta q$ with a shape factor $k=0.9$, as outlined in the chapters 3 and 3.4. The crystallite size along the $y$-axis $(0 \mathrm{k} 0)$ was calculated from the (120)-peak, along with the pre-determined size from the (200)-peak.

$$
L_{0 k 0}=L_{120} \cos \left(\arctan \left(\frac{a}{2 b}\right)\right)=L_{120} \cos \left(29.31^{\circ}\right)
$$

However, since the (200)-peak is only observed as a shoulder to the stronger (120)peak, a clear separation of the lateral width along the $x$ and $y$ axes, respectively, is delicate and subject to significant errors. At zero or low strain, the crystallite size determined for the three species were NS $=67 \times 48 \times-\AA, \mathbf{N M}=50 \times 46 \times-\AA$ and NC $=43 \times 48 \times-\AA$. These values are in good agreement with previous measurements of fiber bundles. The value found for the crystallites length along the $z$-axis in the single fiber experiment was problematic, as discussed below. First we consider $\Delta q_{120}(\varepsilon)$, which behaves alike for all three Nephila species. While the peak positions $q_{120}$ and $q_{200}$ do not change with strain, $\Delta q_{120}(\varepsilon)$ increases linearly, and the lateral crystallite size decreases correspondingly. Up to $8 \%$ strain, $L_{120}$ decreases from $52 \AA$ down to $46 \AA$ corresponding to an average loss of $1.5 \mathrm{Bragg}$ planes. We attribute this loss of more than one lattice plane to a strain induced unfolding or fracturing (splitting) of the $\beta$-sheet crystallites along the direction of the hydrogen-bonds. Next we consider the crystallite size $L_{200}$ calculated from the (200)-reflection. This peak shows a different behavior for different species. There was no strain-dependency observable for NC and NM. For NS $L_{200}$ decreases from $66 \AA$ down to $45 \AA$ at $20 \%$ strain, corresponding to a decrease of two Bragg planes. However, as stated above, the results for the (200)-peak are always very susceptible to the details of the fitting approach (lineshape, parameter initialization). The high covariance with the (120)-peak parameters make it difficult, if not impossible to single out the (200)-peak parameters in a unique manner. We now consider the crystallite's size along the $z$-axis. Unfortunately, only for NM, the (002)-reflection was pronounced enough to allow a quantitative analysis. The width $\Delta q_{002}$ of the (002)-reflection was obtained from meridional slices and cake integrations. Both methods yield consistent results, see table 8.1. The corresponding length $L_{002}$, shows no dependency on the strain, while the corresponding lattice constant $q_{002}$ does, see below. The low signal to noise of this peak leads to significant errors. In absolute numbers, the crystal size $L_{002}$ along $z$ varies between $150 \AA$ and $100 \AA$. Compared to previously published results on fiber bundles, this range of sizes is a factor of two larger, see section 2.2. In other 
words the peak width is about two times broader in fiber bundle than in single fiber measurements, for reasons which are yet unclear.

\subsubsection{Peak Position and Lattice Constants}

The peak positions $q_{120}$ and $q_{200}$ were found to be independent of strain within experimental errors. Thus the lattice constants perpendicular to the direction of the force exhibit no or only a small strain dependence, see also table 8.1. Of all $q$-values $q_{002}$ showed the clearest correlation with strain. However, only for NM this peak was sufficiently intense for analysis. With increasing strain the lattice constant $c$ increases for NM. Up to $9 \%$ strain, there is a nearly linear correlation with a 15:1 ratio. When the fiber is stretched by $5 \%$ of it's initial length, the lattice constant $c$ increases by approximately $0.33 \%$, see the linear fit in figure 8.3a, for the corresponding slope see table 8.1. The off-axis slices support this observation only up to $4 \%$. Above $4 \%$ strain the values of the off-axis slices through the (121)-reflection are quite scattered. Furthermore, in absolute numbers, the lattice constant calculated from the off-axis slices is smaller than the value obtained from $q_{002}$, namely $c_{\text {offaxis }}=6.89 \AA$ compared to $c_{002}=6.952 \AA$. This shift seems to be intrinsic to the diffraction pattern, and may possibly be explained by strain fields or defects. As can be seen for example in figure 3.9, the (122)-peak is also shifted to higher $q$-values with respect to the (002)-peak.

\subsubsection{Amorphous Halo}

In samples with scattering high intensity it is also possible to analyse the scattering contribution of the amorphous matrix, i.e. the disordered polypeptide chains in which the crystallites are embedded and by which they are linked. As for the (002)-reflections, only NM samples showed at signal to noise ratio high enough for analysis. The amorphous halo can be analysed best in the meridional plane where it is not overlapped by scattering from the crystalline fraction. The peak center corresponds to the mean distance $d_{\text {amorph }}=2 \pi / q_{\text {halo }}$ of scatterers in the amorphous matrix, and exhibits a linear strain dependency. Starting with a value of $d_{\text {amorph }}=3.72 \AA$ at $\varepsilon=2 \%$ it decreases to approximately $d_{\text {amorph }}=3.5 \AA$ at $\varepsilon=9 \%$, see also figure $8.3 \mathrm{~b}$ and table 8.1. The width of the amorphous halo $\Delta q_{\text {amorph }}$ exhibits no dependency on $\varepsilon$, see table 8.1. Note that the decrease in the mean distance parallel to the applied strain is difficult to interpret and in contrast to the behavior of the lattice constant $c$. At the moment we don't have a satisfying explanation for this finding. 


\subsection{Discussion}

With these single fiber experiments we have shown that it is possible to observe mechanically-induced structural changes in single dragline fibers by synchrotron X-ray scattering.

It was found that the orientation of the $\beta$-sheet crystallites along the fiber axis, as well as the lattice constant $c$ parallel to the strain direction increases with increasing strain. At the same time the radial width of the reflections along the equatorial axes is found to increase. Since it is unlikely that a strain effect would induce a broadening along the equatorial axes $(x, y)$ but not in the meridional axis $(z)$, we attribute this broadening to a decrease of the crystallite lateral size.

The energy to stretch the fiber can easily be obtained from the force-strain curves, as it corresponds to the area under the curve, see chapter 3.5. We used the upper force values in figure 8.1a because they resemble the values for a force-strain curve without relaxation. Correspondingly, the energy necessary to stretch a single dragline fiber of $30 \mathrm{~mm}$ length up to $8 \%$ lies e.g. around $3.4 \cdot 10^{-2} \mathrm{~mJ}$ for $\mathbf{N M}$. To estimate the energy necessary to split all crystallites in the above mentioned way, the energy to split one crystallite and the total number of crystallites in the fiber have to be estimated. We assume five unit cells along both $x$ - and $y$-axis, and nine unit cells along $z$-axis with eight amino acids in each unit cell and two hydrogen bonds per amino acid. We take two H-bonds, since the weak non-covalent bonds (interaction via van-der-Waals forces) between the amino acids residues of different adjacent sheets can be estimated to have the same energy as the hydrogen bonds ( $20 \mathrm{~kJ}$ per mol). The experimental findings suggest that the crystallite decreases from $5 \times 5 \times 9$ unit cells to $4 \times 4 \times 9$ unit cells. That means 81 unit cells are split along $x$ - and $y$-axis or that 1296 bonds have to be broken.

With these values and a crystalline fraction of $20 \%$ the energy in a $30 \mathrm{~mm}$ fiber with $6 \mu \mathrm{m}$ diameter is $4.67 \cdot 10^{-2} \mathrm{~mJ}$ which is in the same energy range as the total energy for stretching. Naturally this rough estimation depends strongly on the number of broken bonds, the bond energy and on the number of amino acids at the surface. From hysteresis experiments [52] we know that only $68 \%$ of the energy is dissipated. If the crystallites only decrease by breaking hydrogen bonds as could be concluded from the $L_{120}$ versus $L_{200}$ analysis in NM, the splitting energy is reduced to $2.335 \cdot 10^{-2} \mathrm{~mJ}$ which is approximately $69 \%$ of the total energy of $3.4 \cdot 10^{-2} \mathrm{~mJ}$ necessary to stretch the fiber and can be regarded as an upper limit. This rough estimate shows that 'splitting' of the crystallites or better to an exfoliating of $\beta$-sheets at the internal surface of the crystalline and the amorphous regions is a plausible mechanism for energy dissipation [98].

Possibly this exfoliation effect results from shear induced by mechanically 'loaded' protein strands. Due to the small size of the crystallites the internal interfaces in a fiber exceed the external surface by huge amount, namely by factor on the order of $L / D \simeq 10^{3}$. Mechanical properties, including viscoelasticity, as well as transport properties and the uptake of solvents may be significantly be influenced by the properties of these interfaces. Under mechanical load polypeptides at these interfaces may then transform form the crystalline to an amorphous state, in agreement with the observed size reduction of the crystallites. 
This interpretation is also supported by the results of hysteresis measurements [52; 53], which shows that the envelope of repeated hysteresis cycles forms a "normal" force-strain curve [52]. This is an indication for irreversible processes in the fiber during stretching, which could also be attributed to an exfoliation of crystallites, as described above.

A further key for the understanding of the mechanical properties could be the variation of properties with relative humidity. The stress-strain curves show a pronounced transition with relative humidity $[52 ; 53]$, see figure $8.1 \mathrm{~b}$. Note that a similar behavior is known for synthetic polyamides such as nylon 6,6 which show a similar behavior when wetted [81]. It is plausible that the water diffuses into the amorphous parts of the fibers and replaces hydrogen-bonds between the polymer strands, such that the system softens. This transition induced by changes in the amorphous matrix (where water uptake is possible) indicates the importance of the amorphous matrix for the extensibility $\varepsilon_{\max }$ and the initial elastic modulus $\mathcal{E}$, whereas the irreversible changes related to energy dissipation would take place primarily in the crystallites, which at the same time also reinforce the matrix. Investigations of structural changes as a function of both external strain and relative humidity may shed further light on these issues. Finally, it would be important to further characterize the structural properties of the amorphous matrix, which however seems difficult due to the predominance of the crystalline components in the scattering intensity distribution. 

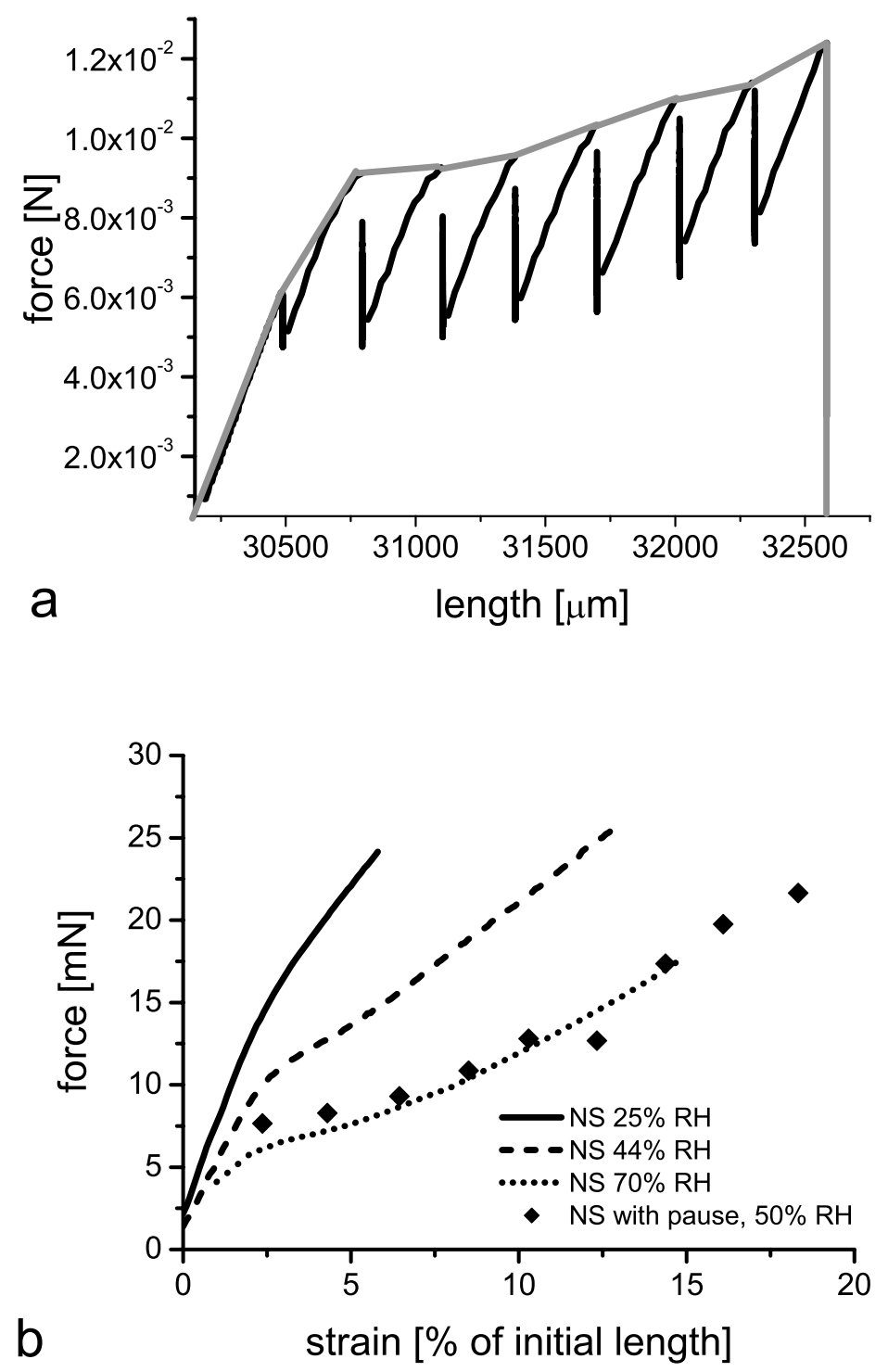

Figure 8.1: (a) Force-strain curve for a NM fiber. The initial length of the fiber was 30mm. The cusps in the curve reflect the pauses during stretching, at which X-ray data accumulation took place. The enveloppe of the curve connecting the upper force values is equivalent to a force-strain curve without pauses and was used to calculate the energy necessary to stretch the fiber (area under the curve).(b) Force-strain curves measured at different relative humidities (lines). Data is shown for NS samples. The lines correspond to continuous measurements [52; 53]. The symbols $(\diamond)$ indicate the force-strain values measured with breaks in the elongation at the microfocus X-ray experiment. 

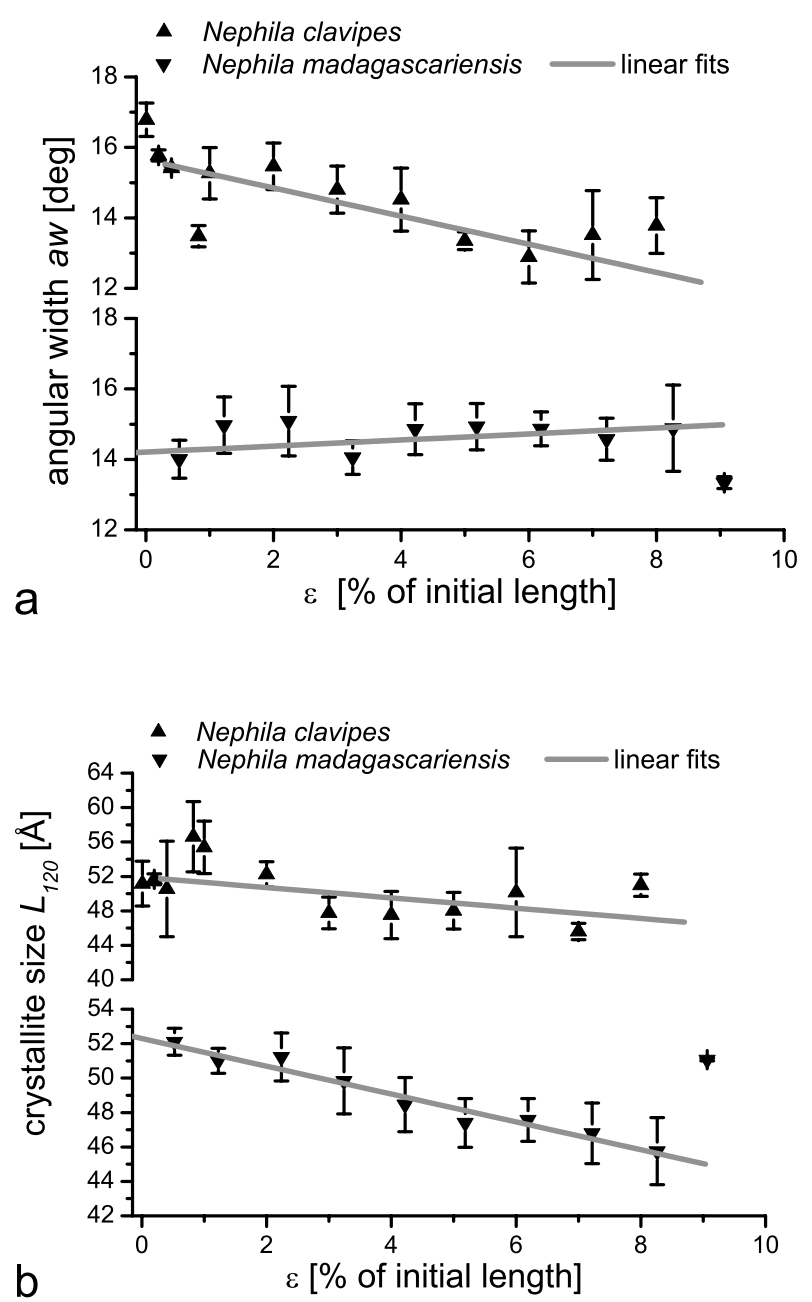

Figure 8.2: (a) Angular width aw of NM and NC samples versus strain. For NC the decrease in aw indicates a significient increase in crystallite orientation, while a constant relationship is observed for NC . (b) Crystallite size $L_{120}$ as a function of strain $\varepsilon$ calculated from the width of the (120)-peak. The data shows that the crystallite size perpendicular to the direction of the strain, decreases. 

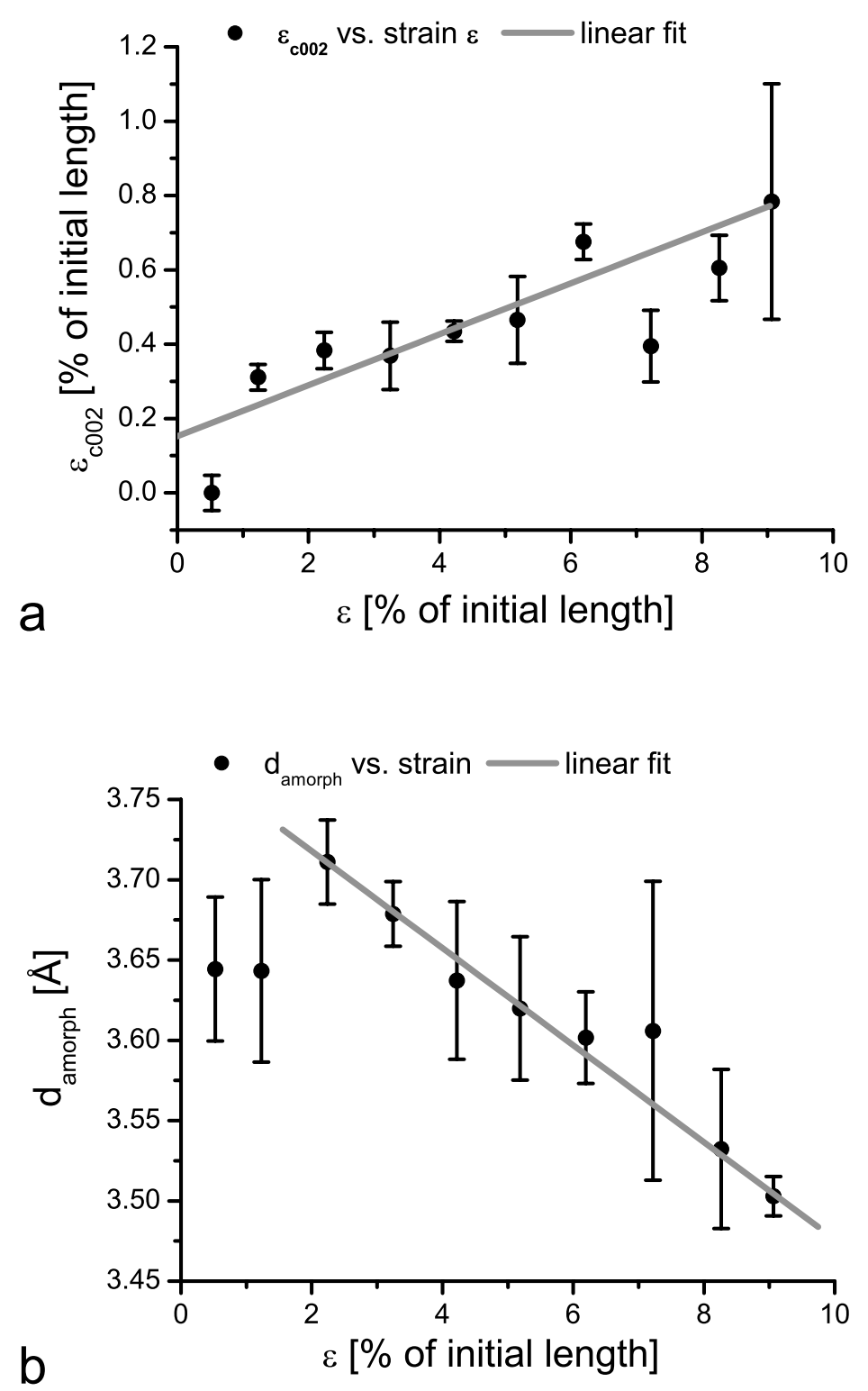

Figure 8.3: (a) The strain lattice $\varepsilon_{c 002}$ calculated from the relative changes of the lattice constant $c$, as obtained from the (002)-peak (shown for NM), as a function of external strain $\varepsilon$. (b) Analysis of the amorphous halo originating from the disordered chains. The mean distance of the amorphous matrix $d_{\text {amorph }}$ parallel to the fiber axis decreases from $2 \%$ strain on. 


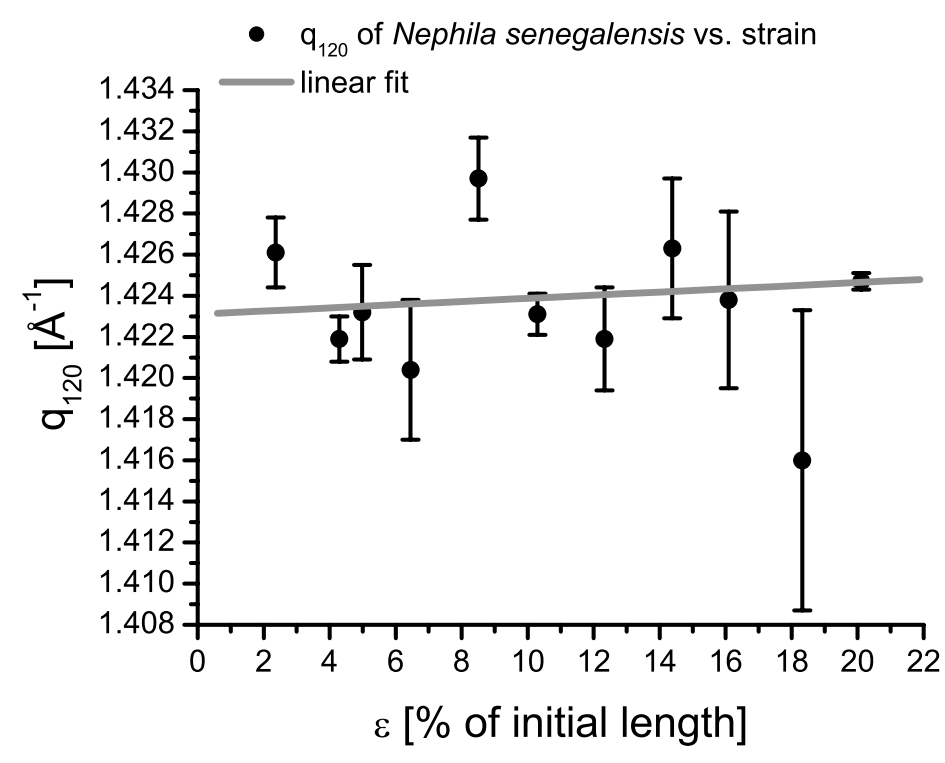

Figure 8.4: Position of the equatorial (120)-peak $q_{120}$ from $N$. senegalensis versus strain. Despite the high relative elongation of $\varepsilon=20 \%$ the peak position stays nearly constant.

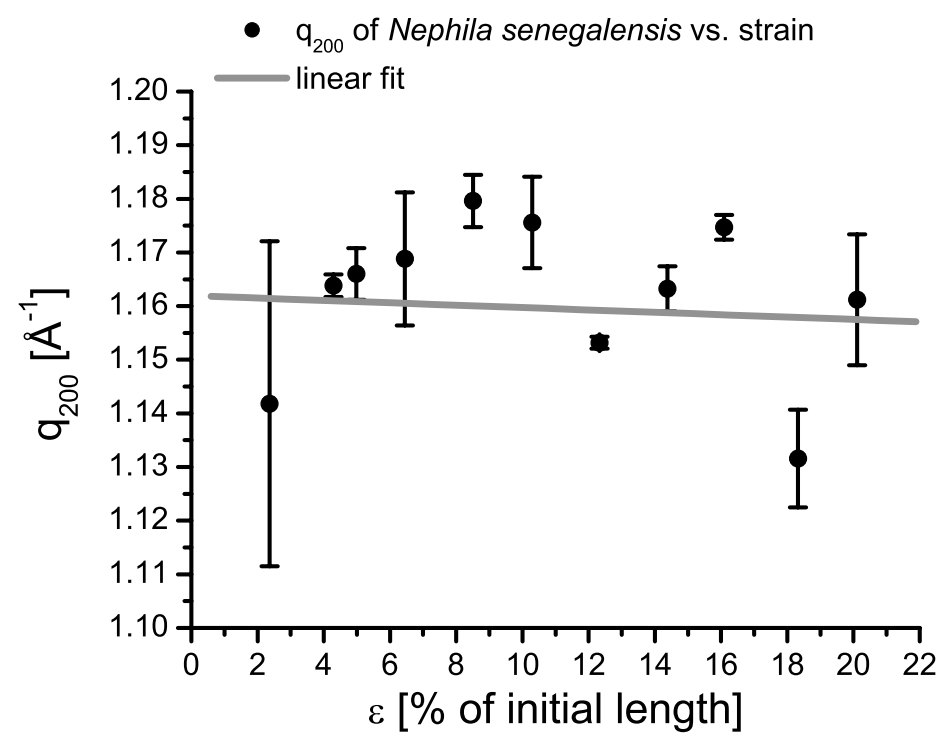

Figure 8.5: Position of the equatorial (200)-peak $q_{200}$ from $N$. senegalensis versus strain. As for the (120)-peak the peak's position stays nearly the same through out the whole elongation of $\varepsilon=$ $20 \%$ 


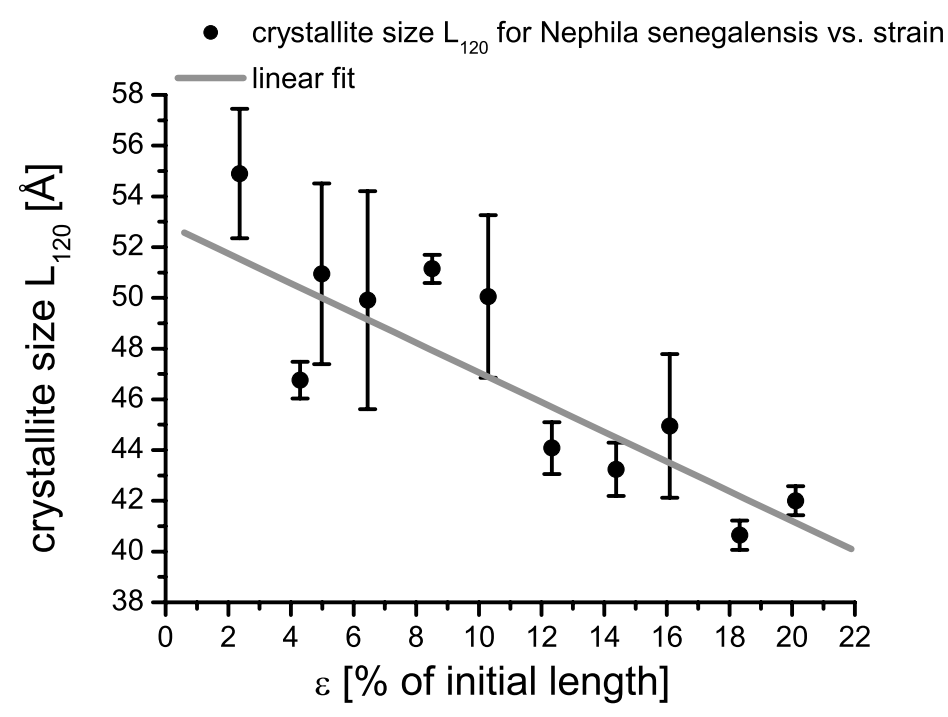

Figure 8.6: Crystallite size $L_{120}$ for $\mathrm{N}$. senegalensis versus strain. The size was calculated via Debye-Scherrer formula from the peak's fwhm. $L_{120}$ decreases with increasing strain from $55 \AA$ at $\varepsilon=0.5 \%$ to $42 \AA$ at $\varepsilon=20 \%$ strain. This corresponds to a decrease of nearly three Bragg planes and is similar to the finding on $\mathrm{N}$. madagascariensis dragline.

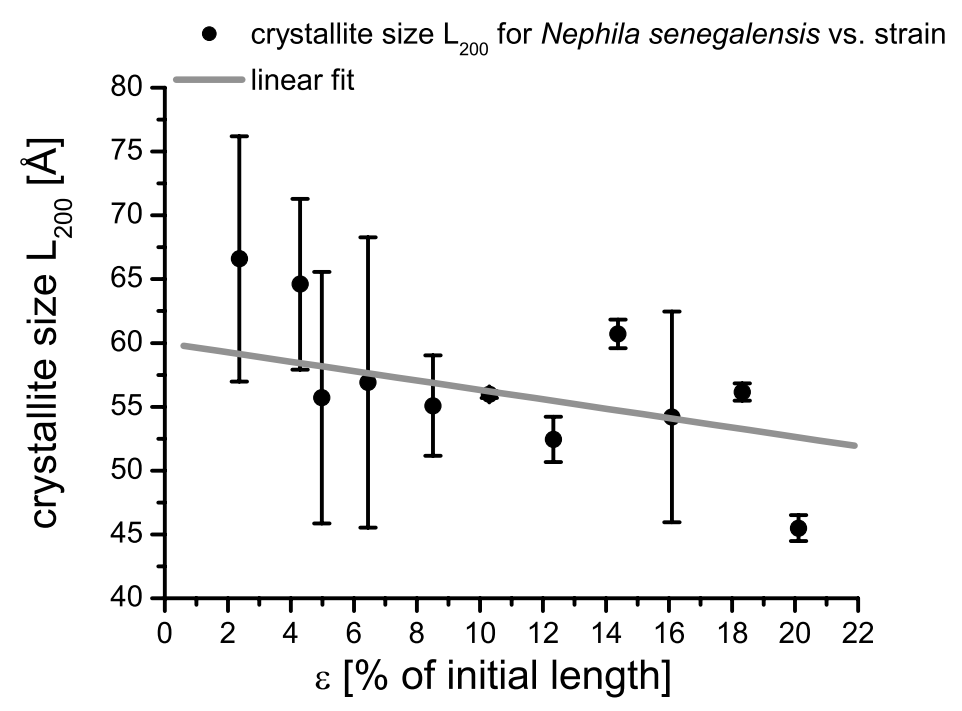

Figure 8.7: Crystallite size $L_{200}$ for $N$. senegalensis versus strain. The size was calculated via Debye-Scherrer formula from the peak's fwhm. L200 decreases with increasing strain from $66 \AA$ at $\varepsilon=0.5 \%$ to $45 \AA$ at $\varepsilon=20 \%$ strain. This corresponds to a decrease of nearly four Bragg planes and is not found that pronounced in the dragline of the other studied species. 


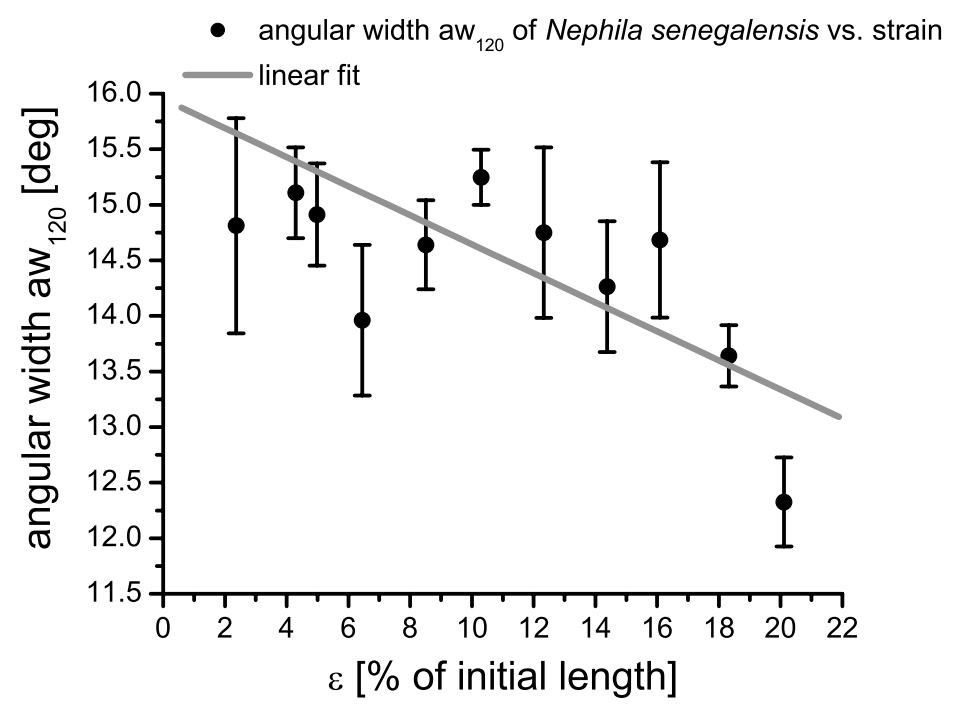

Figure 8.8: Angular width of the (120)-reflection aw $w_{120}$ for N. senegalensis versus strain. Comparable to $\mathrm{N}$. clavipes the angular width decreases with increasing strain, the order of the crystallites increases. 
Table 8.1: Values from linear regression of different crystallite parameters. Linear regression was performed in an interval of 0.5-8\% strain for NM and 1-8\% strain for NC. $q_{h k l}$ peak position and $L_{h k l}$ crystallite length calculated from fwhm; aw $w_{120}$ angular width from arc slices, shown in figure 3.10c; $c_{002}$ z-axis' lattice constant calculated from meridional slices, shown in figure $3.10 b ; c_{002}^{\text {cake }}$ z-axis' lattice constant calculated from cake integration, shown in figure 3.10d; $c_{\text {offaxis }}$ z-axis' lattice constant calculated form slices as shown in figure 3.10e. The linear regime of the mean distance $d_{\text {amorph }}$ starts at $2 \%$ strain. The cake integration was only performed for NMdue to its sufficient intensity of the amorphous halo.

\begin{tabular}{|c|c|c|c|c|c|c|}
\hline \multirow[t]{2}{*}{$x$} & \multicolumn{2}{|c|}{$\mathrm{NC}$} & \multicolumn{2}{|c|}{ NM } & \multicolumn{2}{|c|}{ NS } \\
\hline & intercep & $t$ slope & intercep & t slope & intercep & slope \\
\hline$q_{120}[\AA]^{-1}$ & 1.423 & $7.44 E-4$ & 1.423 & $-6.74 E-5$ & 1.423 & $7.71 E-5$ \\
\hline error & $\pm 9.99 E-4$ & $\pm 2.44 E-4$ & $\pm 4.35 E-4$ & $\pm 1.34 E-4$ & $\pm 9.02 E-4$ & $\pm 5.25 E-4$ \\
\hline$q_{200}[\AA]^{-1}$ & 1.170 & $-1.30 E-4$ & 1.185 & $-8.22 E-4$ & 1.162 & $-2.22 E-4$ \\
\hline error & $\pm 2.85 E-3$ & $\pm 6.44 E-4$ & $\pm 1.04 E-3$ & $\pm 2.70 E-4$ & $\pm 2.65 E-3$ & $\pm 2.22 E-4$ \\
\hline$q_{002}[\AA]^{-1}$ & & & 1.807 & $-1.21 E-3$ & & \\
\hline error & & & $\pm 5.43 E-4$ & $\pm 1.34 E-4$ & & \\
\hline $\begin{array}{l}q_{\text {cake }}[\AA]^{-1} \\
\text { error }\end{array}$ & & & $\begin{aligned} & 1.815 \\
\pm & 3.75 E-4\end{aligned}$ & $\begin{array}{l}-1.17 E-3 \\
\pm 1.12 E-4\end{array}$ & & \\
\hline $\begin{array}{l}q_{\text {halo }}[\AA]^{-1} \\
\text { error }\end{array}$ & & & $\begin{aligned} & 1.660 \\
\pm & 1.06 E-2\end{aligned}$ & $\begin{array}{r}1.46 E-2 \\
\pm 1.52 E-3\end{array}$ & & \\
\hline$L_{120}[\AA]$ & 51.91 & -0.60 & 52.30 & -0.81 & 52.91 & -0.59 \\
\hline error & \pm 1.48 & \pm 0.25 & \pm 0.60 & \pm 0.16 & \pm 0.67 & \pm 0.05 \\
\hline$L_{200}[\AA]$ & 52.98 & -0.29 & 49.12 & 0.23 & 60.01 & -0.37 \\
\hline error & \pm 1.06 & \pm 0.26 & \pm 0.87 & \pm 0.16 & \pm 0.89 & \pm 0.07 \\
\hline$L_{002}[\AA]$ & & & 142.56 & -4.28 & & \\
\hline error & & & \pm 4.97 & \pm 0.95 & & \\
\hline $\begin{array}{l}L_{\text {cake }}[\AA] \\
\text { error }\end{array}$ & & - & $\begin{array}{r}158.27 \\
\pm 4.29\end{array}$ & $\begin{array}{l}-3.48 \\
\pm 0.95\end{array}$ & & \\
\hline$a w_{120}[\mathrm{deg}]$ & 15.65 & -0.40 & 14.20 & 0.09 & 15.95 & -0.13 \\
\hline error & \pm 0.56 & \pm 0.12 & \pm 0.43 & \pm 0.09 & \pm 0.30 & \pm 0.02 \\
\hline $\begin{array}{l}\mathcal{c}_{002}[\AA] \\
\text { error }\end{array}$ & & & $\begin{aligned} & 6.952 \\
\pm & 2.10 E-3\end{aligned}$ & $\begin{array}{r}4.76 E-3 \\
\pm 5.26 E-4\end{array}$ & & \\
\hline $\begin{array}{l}c_{\text {cake }}[\AA] \\
\text { error }\end{array}$ & & & $\begin{aligned} & 6.925 \\
\pm & 1.45 E-3\end{aligned}$ & $\begin{array}{r}4.50 E-3 \\
\pm 4.31 E-4\end{array}$ & & \\
\hline $\begin{array}{l}c_{\text {offaxis }}[\AA] \\
\text { error }\end{array}$ & $\begin{array}{l}6.902 \\
\pm 3.54 E-2\end{array}$ & $\begin{array}{r}8.75 E-3 \\
\pm 8.43 E-3\end{array}$ & $\begin{array}{l}6.897 \\
\pm 3.57 E-3\end{array}$ & $\begin{array}{l}-1.71 E-5 \\
\pm 1.55 E-3\end{array}$ & & \\
\hline $\begin{array}{l}d_{\text {amorph }}[\AA] \\
\text { error }\end{array}$ & & - & $\begin{array}{l}3.726 \\
\pm 2.51 E-2\end{array}$ & $\begin{array}{l}-1.91 E-2 \\
\pm 5.85 E-3\end{array}$ & & \\
\hline $\begin{array}{l}\Delta q_{\text {amorph }}[\AA]^{-1} \\
\text { error }\end{array}$ & & - & $\begin{array}{l}1.817 \\
\pm 6.19 E-2\end{array}$ & $\begin{array}{l}-9.69 E-3 \\
\pm 1.29 E-2\end{array}$ & & \\
\hline
\end{tabular}





\section{Chapter 9}

\section{Single Fiber Diffraction - The Effect of High Humidities and Strain}

To study the influence of strain and humidity on the dragline's structure an experiment was performed at the ESRF's microfocus beamline ID13, similar to the one described in chapter 8. For humidity control the LEX 810 extensometer was equipped with the humidity chamber described in section 3.5.1 which allowed measurements below 5\% RH and above $90 \% \mathrm{RH}$. To compare the structure at low and high humidities the fiber was placed in the extensometer and exposed to dry nitrogen $(\mathrm{RH}<5 \%)$. After $30 \mathrm{~min}$ of equilibration the fiber was strained to $0.5 \%$ and a diffraction pattern was recorded. Then the fiber was relaxed, humidity increased over $90 \% \mathrm{RH}$. Again the sample was given $30 \mathrm{~min}$ for equilibration and a new diffraction pattern at $\varepsilon=0.5 \%$ and $90 \%$ humidity was recorded. Additionally, fibers previously immersed in water, were measured at humidities above $90 \% \mathrm{RH}$. All samples were approximately $30 \mathrm{~mm}$ long when inserted into the extensometer. Due to supercontraction, see chapter 2.4, the sample preparation for the immersed samples had to be modified. The fibers were glued at one side to a sample holder, cut to a length of 7-8cm and pulled underwater using the sample holder as a handle. The samples were kept in the MilliQ water for 5-10min. Then the supercontracted fibers were prepared as described in chapter 4, mounted in extensometer and the humidity chamber, which was set for humidities above $90 \%$ $\mathrm{RH}$.

When the experiments were performed the microfocus beamline was equipped with CRLs for beam focusing, see chapter 3.3.2. The advantage of this setting was a much cleaner beam, the disadvantage a lower flux which made an increase of the exposure time from 11 seconds to one minute per pattern necessary. Therefore it was unnecessary to take a series of diffraction patterns for one strain value at different positions of the fiber. The longer illumination time would have increased the time for such a series inadequately in respect to the limited beamtime.

The option of relaxation measurements was used for the extensometer settings. This means the sample was expanded up to a preset strain value (e.g. $0.5 \%$ for alignment and first measurements) and kept at this strain for 10min. In this time the force was recorded. After the preset relaxation time the extensometer moved 
back to its starting position. Then the next strain value was encountered. As seen in chapter 5 the gauge force $F_{G}$ in combination with the dragline's 'stretching memory' causes a shift in the measurements starting point. E.g. if the first and second extension of a $30 \mathrm{~mm}$ dragline sample were set to be $0.5 \%$, the first elongation of the fiber will correctly be $0.15 \mathrm{~mm}$ but $0.3 \mathrm{~mm}$ or $1 \%$ for the second extension. This is due to dragline silk's ability to store its stretching history, see also section 2.4. In this way 26 NM fibers and four NC fibers were studied.

\subsection{Influence of Humidity on the Structure at Low Strains}

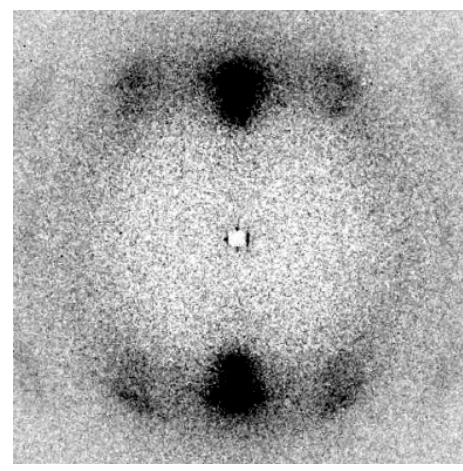

$\mathrm{RH}<5 \%$

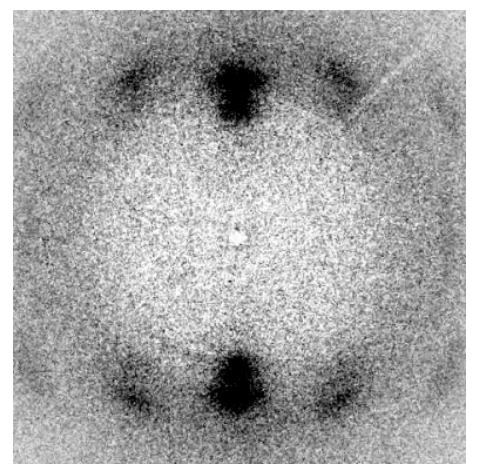

$\mathrm{RH}>90 \%$

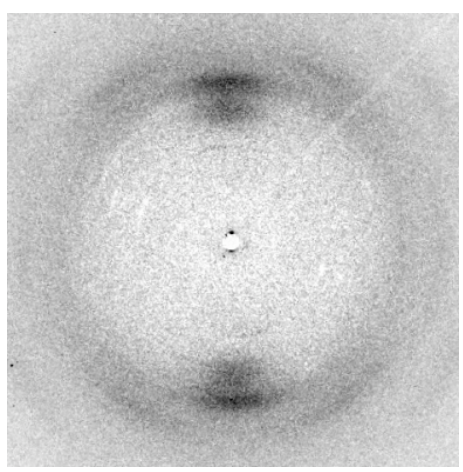

after immersion

Figure 9.1: Comparison of diffraction patterns obtained from N. madagascariensis. The increase of the humidity causes no visible changes in the diffraction patterns. Immersion in water on the other hand causes a circular broadening of the reflections.

In figure 9.1 the diffraction patterns of NM dragline at different humidities are shown. The two patterns taken at different relative humidities are from the same fiber. The pattern of the immersed fiber is from another sample of the same spider species. Visually the higher humidity seems to induce only minor changes in the diffraction pattern while immersion in water causes a circular smearing of the reflections. Out of the ensemble of $26 \mathrm{NM}$ and four NC fibers, only three series for NM provided enough data to measure the structures at low strain as well as at high and low humidities. Only one fiber survived a further elongation to $2 \%$ strain after increasing the humidity. So no systematic study of strain induced structural changes at high humidities was possible. Only the changes at low strain caused by increasing humidity could be recorded. We attribute this poor yield to supercontraction. Although the fibers were relaxed during the rise of humidity the gap of $28 \mathrm{~mm}$ of the LEX 810 extensometer was not enough to allow the fiber a free contraction, see figures 9.2 and 9.3. The longer it is exposed to the humid nitrogen the stronger it contracts until it is saturated, then relaxation occurs. When the contraction is hindered as for the present experiment an internal strain occurs in the fiber. So an additional stretching of the fiber, as necessary for a series of strain values, easily exceeds the maximum strain a sample can bear. 
This explanation is also supported by the angular width at different humidities. In figure 9.8 the angular width is shown for the described humidity conditions and low strain. For humidities smaller than 5\% RH the angular width lies around $16^{\circ}$, for humidities above $90 \% \mathrm{RH}$ around $14^{\circ}$ and for immersed fibers between $25^{\circ}$ and $30^{\circ}$. The decrease of the angular width with increasing humidity is astonishing, especially in the light of the nearly doubled angular width after immersion in water. We found a similar decrease in the experiments at ambient conditions, where the angular width starting point was also around $16^{\circ}$ and decreased down to approximately $13^{\circ}$ at a strain of $\varepsilon=6 \%$ for NC. So it seems appropriate to assume that the suppressed supercontraction induced an internal stress in the NC fiber [54] of approximately $34 \mathrm{MPa}(F(\varepsilon=6 \%)=6.75 \mathrm{mN}, 5 \mu \mathrm{m}$ diameter $)$ corresponding to an external strain of $6 \%$ at ambient conditions.

Further it could be observed that the crystallite size changes with humidity anisotropic for the equatorial reflections, see table 9.1. $L_{120}$ decreases from approximately $54 \AA$ to $43 \AA$, which corresponds to a loss of two Bragg planes, while $L_{200}$ increases from nearly $40 \AA$ to $47 \AA$., which corresponds to 1.4 Bragg planes. This can most likely be attributed to the different types of bonds along the crystal axes, see section 2.2. The (200)-reflections is sensitive to the hydrophobic interactions of the alanine residues along the $x$-axis which can increase crystallite size by $7 \AA$. These bonds also contribute to the (120)-reflection, but the peak is also influenced by hydrogen bonds along the $y$-axis. Therefore it can be assumed that similar to the amorphous matrix the H-bonds along this axis are partly suppressed by the water permeated into the fiber. What causes a deceases in the crystallite size along the $y$-axis $L_{0 k 0}$ from $47 \AA$ to $38 \AA$. Nevertheless the product of the crystallite sizes $L_{200}$ and $L_{120}$ is nearly the same for both dry and wet states. With respect to the model of Y. Termonia, section 2.5.2, this seems likely because the crystallite size strongly influences the fibers toughness which is also nearly the same for wet and dry states, see chapter 5, tables 5.1 and 5.2.

Table 9.1: Crystal parameters at low strains $\varepsilon \leq 1 \%$ and different humidities obtained from $\mathrm{N}$. madagascariensis dragline.

\begin{tabular}{cccc}
\hline parameter & $\leq 5 \% \mathrm{RH}$ & $\geq 90 \% \mathrm{RH}$ & immersed \\
\hline$q_{120}\left[\AA^{-1}\right]$ & $1.426 \pm 1.71 E-3$ & $1.422 \pm 1.23 E-3$ & $1.423 \pm 3.70 E-3$ \\
$q_{200}\left[\AA^{-1}\right]$ & $1.168 \pm 1.02 E-3$ & $1.171 \pm 3.55 E-3$ & $1.178 \pm 4.20 E-3$ \\
\hline$L_{120}[\AA]$ & $53.55 \pm 1.38$ & $58.68 \pm 1.35$ & $43.22 \pm 3.53$ \\
$L_{200}[\AA]$ & $39.79 \pm 1.22$ & $42.76 \pm 1.98$ & $47.15 \pm 2.22$ \\
\hline$a w_{120}[\mathrm{deg}]$ & $16.00 \pm 1.17 E-1$ & $14.18 \pm 1.02 E-1$ & $31.16 \pm 8.89 E-1$ \\
\hline
\end{tabular}




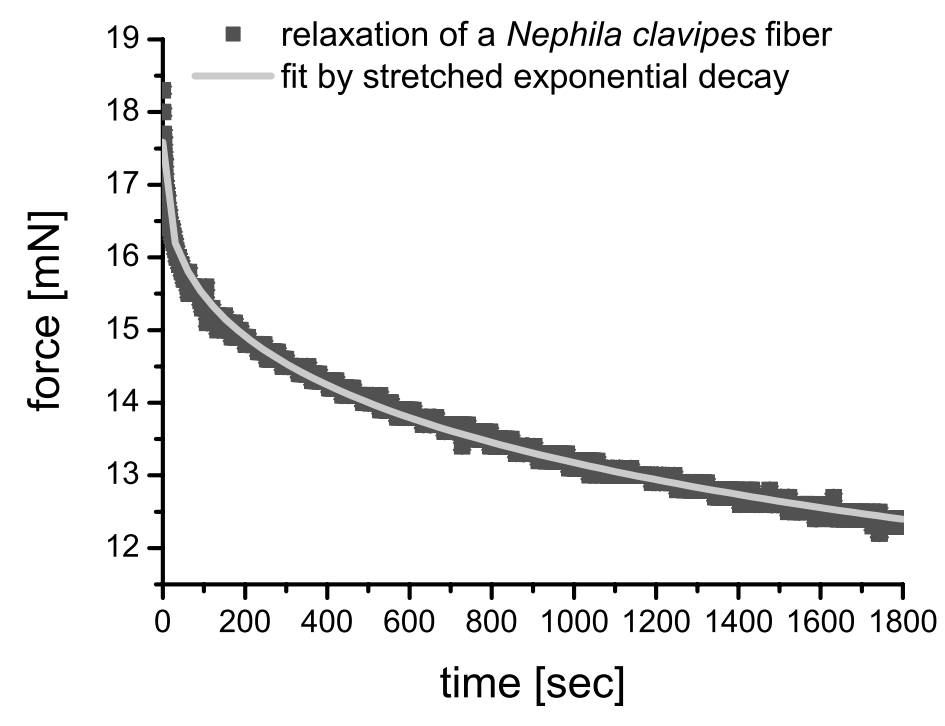

Figure 9.2: Relaxation curve of a N. clavipes dragline at 40\% RH. The fiber was extended to $\varepsilon=5 \%$ of it's initial length, the force-time relation was recorded over $1800 \mathrm{sec}$. The force-time curve can be fitted by a stretched exponential decay $F(t)=F_{1}+F_{2} \cdot \exp \left(-(t / \tau)^{\beta}\right.$, see chapter 5. Data by T. Vehoff [52; 53].

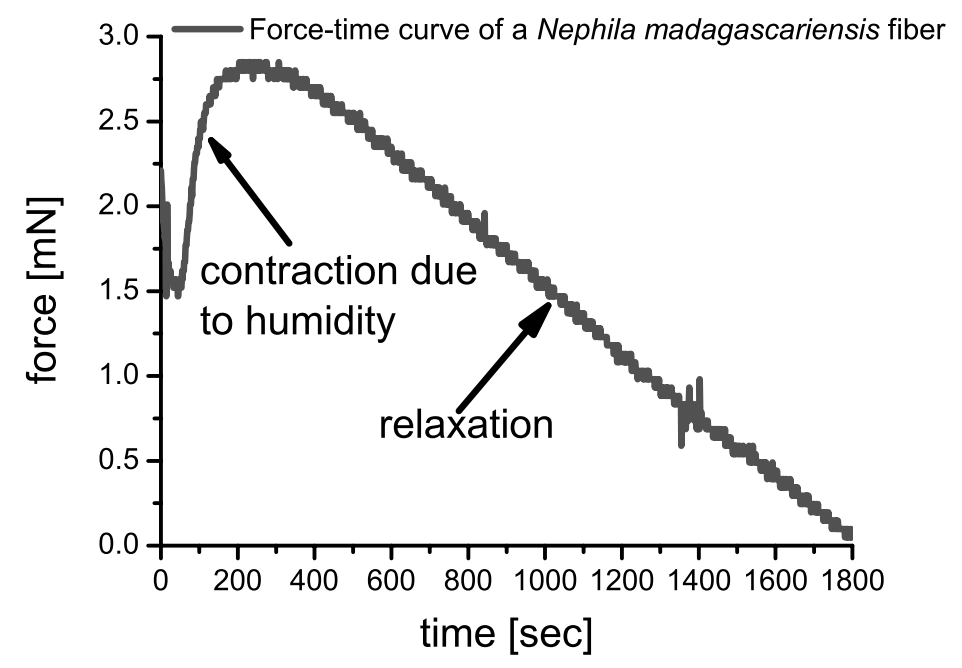

Figure 9.3: Force versus time for a N. madagascariensis dragline sample. The fiber was stored at $50 \% \mathrm{RH}$, in the chamber the humidity was above $90 \% \mathrm{RH}$, the fiber kept at $\varepsilon=0.5 \%$ and the force-time curve recorded over $1800 \mathrm{sec}$. In the first $30 \mathrm{sec}$ the fiber relaxes, with enduring uptake of humidity it starts to contract (30 to 230sec). The force rises from $1.52 \mathrm{mN}$ to $2.85 \mathrm{mN}$. After the fiber is saturated a linear relaxation starts $(t<230 \mathrm{sec})$ and not the normal exponential decay as shown in figure 9.2. 


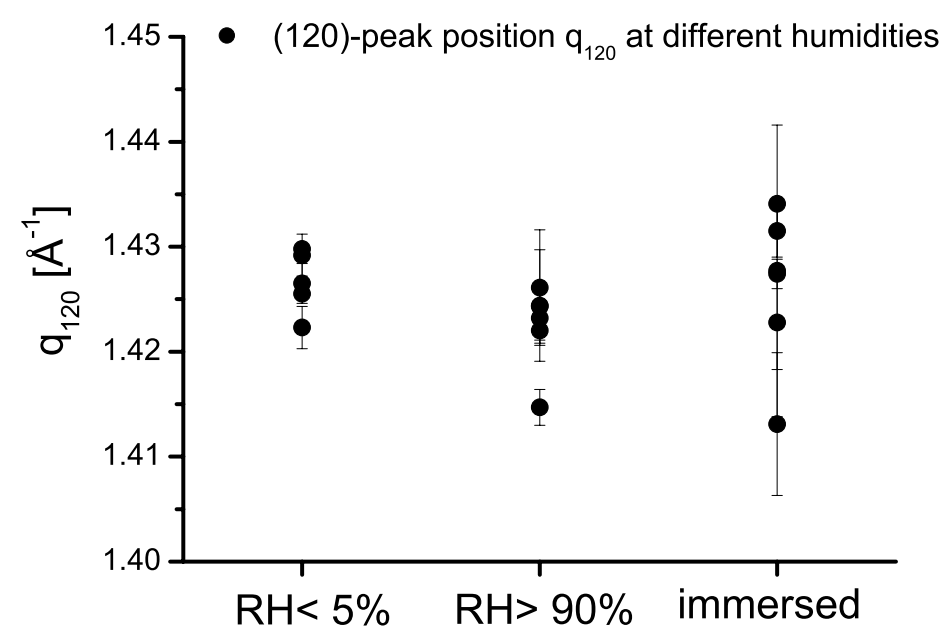

Figure 9.4: Equatorial (120)-reflection's positions $q_{120}$ measured at three different humidity conditions and strains $\varepsilon \leq 1 \%$. The values of all measurements are shown. A precise correlation between peak position $q_{120}$ and humidity is hard to estimate. The ensemble's deviation increases with humidity.

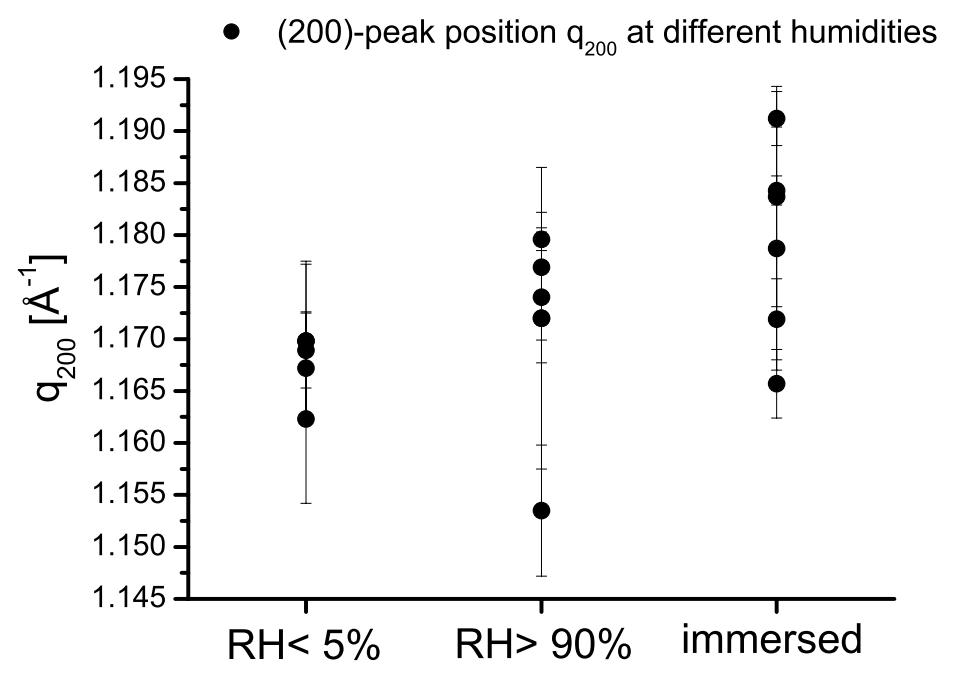

Figure 9.5: Equatorial (200)-reflection's positions $q_{200}$ measured at three different humidity conditions and strains $\varepsilon \leq 1 \%$. The values of all measurements are shown. The position $q_{200}$ increases with humidity, so the lattice constant a decreases, corresponding to the hydrophobic nature of the alanine residues along this axis. 


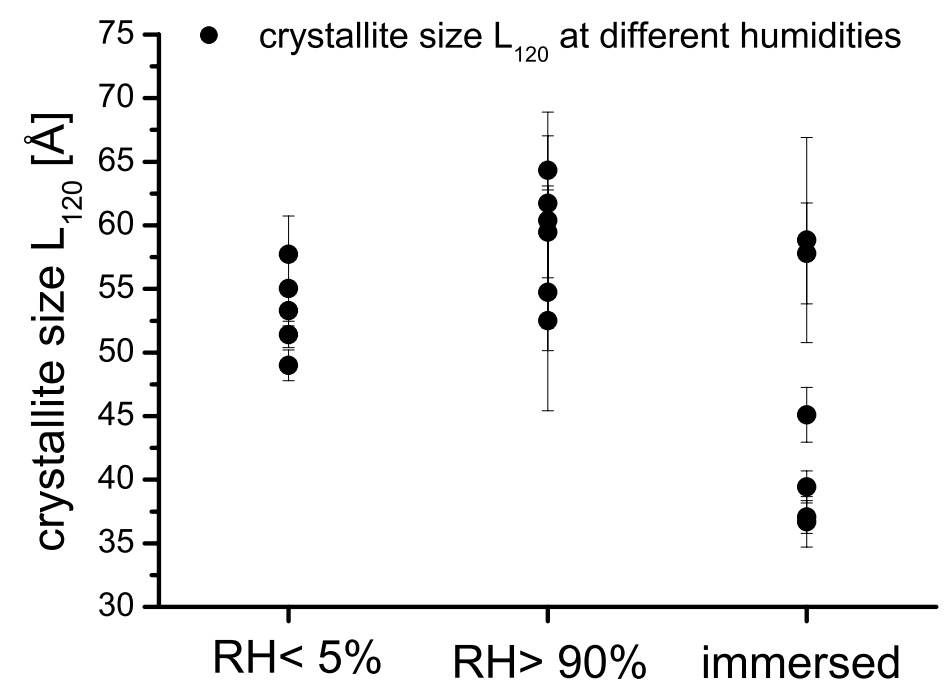

Figure 9.6: The crystallite size $L_{120}$ of the equatorial (120)-reflection for three different humidity conditions measured at strains $\leq 1 \%$. All measurements are shown. The crystallite size changes $L_{120}$ from approximately $54 \AA$ under arid conditions to $43 \AA$ after immersion in MilliQ water.

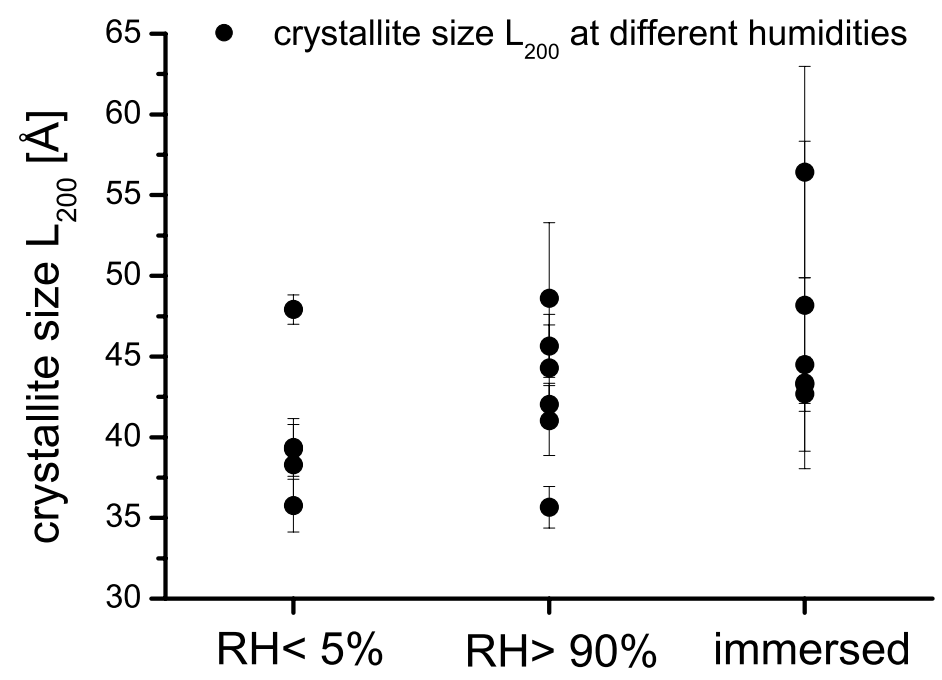

Figure 9.7: The crystallite size $L_{200}$ for three different humidity conditions measured at strains $\leq$ $1 \%$. The values of all measurements are shown. The crystallite size $L_{200}$ increases from approximately $40 \AA$ under arid conditions to $47 \AA$ after immersion in MilliQ water. This corresponds to a gain of nearly 1.4 Bragg planes. 


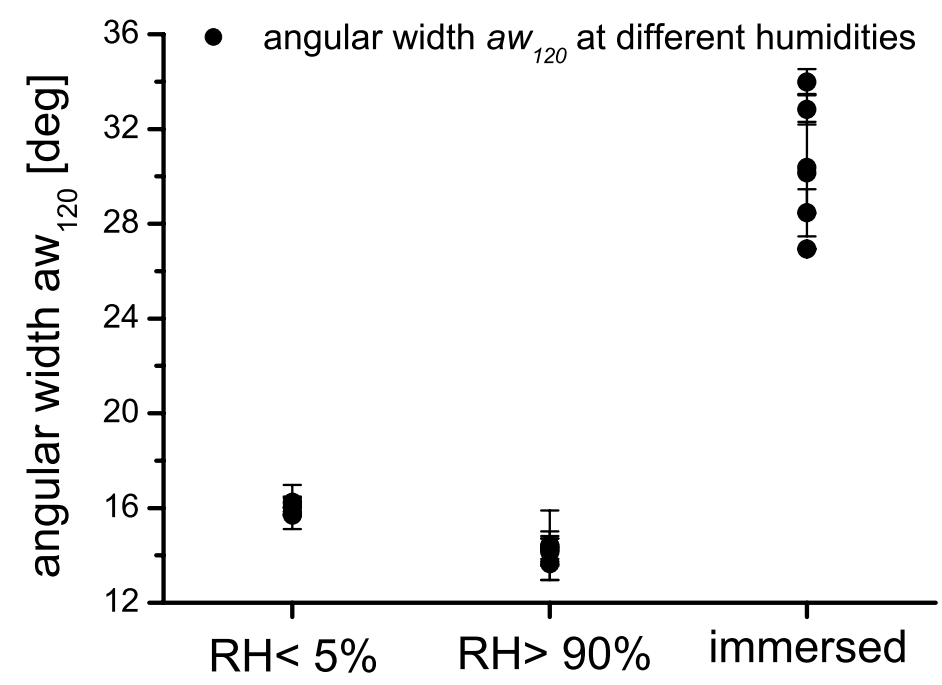

Figure 9.8: The angular width aw $w_{120}$ of the equatorial (120)-reflection for three different humidity conditions measured at strains $\leq 1 \%$. All measurements are shown. The slight decrease from $a w_{120}=16^{\circ}$ at arid conditions to $a w_{120}=14^{\circ}$ at humid conditions we attribute to a hindered supercontraction of the samples. 


\subsection{Immersed Fibers under Strain}

Similar to the experiments presented in chapter 8 the strain induced structural changes of immersed fibers will be adressed here. The error bars for NM indicate the standard error of mean $=$ standard deviation $/ \sqrt{n}$, where $n$ is the number of independent measurements for each strain value $\varepsilon$ while for NC only one sample could be elongated over a sufficient extension range. The NC error bars therefore indicates the fitting errors.

\subsubsection{Angular Width and Crystallite Orientation}

From arc slices through the (120)-peak the angular distribution $a w_{120}$ of the intensity is obtained. This intensity distribution reflects the distribution of the tilt angles $\phi$ of the local $\beta$-sheet $z$-axis with the fiber axis. The order of the crystallites is decreased after immersion reflected by the higher angular width of approximately $24^{\circ}$ compared to $16^{\circ}$ in the arid state, see also tables 9.1 and 9.2. The angular width $a w_{120}(\varepsilon)$ was analysed by a linear regression. Within the experimental errors, it is constant for $\mathbf{N M}$ but decreases for NC by approximately $3^{\circ}$ down to $23.5^{\circ}$ at $\varepsilon=34 \%$, see figures 9.15 and table 9.2. So the crystallites of NC and NS behave as predicted for filled elastomers [99].

\subsubsection{Radial Width and Crystallite Size}

From the fwhm (radial width) measured along the equatorial slice the crystallite sizes $L_{120}$ and $L_{200}$ were calculated by the Debye-Scherrer formula (equation 3.21, $29)$. The crystallite size along the $y$-axis (0k0) was calculated from the (120)-peak, along with the pre-determined size from the (200)-peak.

$$
L_{0 k 0}=L_{120} \cos \left(\arctan \left(\frac{a}{2 b}\right)\right)=L_{120} \cos \left(29.31^{\circ}\right)
$$

Since the (200)-peak is only observed as a shoulder to the stronger (120)-reflection, a clear separation of the lateral width by peak fitting is delicate and subject to significant errors.

At zero or low strain, the crystallite size determined for the two species were $\mathbf{N M}=43 \times 43 \times-\AA$ and $\mathbf{N C}=44 \times 49 \times-\AA$. Up to $28 \%$ strain, $L_{120}$ for NM decreases from $59 \AA$ down to $44 \AA$ corresponding to an average loss of 3.5 Bragg planes and for NC $L_{120}$ decreases from $49 \AA$ down to $37 \AA$ corresponding to an average loss of 3 Bragg planes. The crystallite size $L_{200}$ calculated from the (200)reflection shows a different behavior. There was no strain-dependency observable for NM. For NC the linear regression gives a slope of $0.27( \pm 0.11) \cdot \varepsilon$ which is problematic because it is dominated by the first and the last value which are both around $61 \AA$ while the two values in between are around $45 \AA$.

\subsubsection{Peak Position and Lattice Constants}

The peak positions $q_{120}$ and $q_{200}$ were found to be independent of the applied strain within experimental errors. Thus the lattice constants perpendicular to the 
direction of the force exhibit no or only a small strain dependence, see also table 9.2. This corresponds to findings for ambient conditions.

\subsubsection{Amorphous Halo}

The higher disorder of immersed dragline has the effect that the meridional (002)reflection does no longer overlay the amorphous halo, see figure 9.1. Therefore the amorphous halo was analysed in the meridional slices and no cake integration was necessary. The amorphous peak center corresponds to the mean distance $d_{\text {amorph }}=2 \pi / q_{\text {halo }}$ of the scatterers in the amorphous matrix, and exhibits a linear strain dependency which is a more distinct for NC. Starting with a value of $d_{\text {amorph }}=3.87 \AA$ at $\varepsilon=1 \%$ it decreases to approximately $d_{\text {amorph }}=3.55 \AA$ at $\varepsilon=$ $34 \%$, see also table 9.2. The width of the amorphous halo $\Delta q_{\text {amorph }}$ exhibits a similar dependency on the strain $\varepsilon$ which was not found for the ambient experiments.

\subsection{Discussion}

With these experiments we have shown that it is possible to observe mechanically and humidity-induced structural changes in single dragline fibers by X-ray diffraction.

So it was found that the orientation of the $\beta$-sheet crystallites along the fiber axis decreases significantly with humidity and increases with strain for NC while the strain does not influence the crystallites' orientation in NM fibers. Furthermore it does not influence the equatorial peak positions $q_{120}$ and $q_{200}$. At the same time the radial width of the equatorial (120)-reflection is found to increase. As in chapter 8 we attribute this broadening to a decrease of the crystallite lateral size $L_{120}$. This decrease in $L_{120}$ is discontinuous and the main changes occur at the step from $\varepsilon=15 \%$ to $\varepsilon=28 \%(\mathbf{N M})$ and $\varepsilon=22 \%$ to $\varepsilon=34 \%$ (NM). The findings support those of the experiments at ambient condition, presented in chapter 8 , but the dependencies seemed to be weaker than those at ambient conditions. Most likely this is a result of the supercontraction. The immersed fibers are softer and less ordered, see chapter 5 , so the induced stress by elongation is less than in the uncontracted state. At the same time the humidity influences the crystallite's tilt distribution and the crystallite size. The changes of the average crystallite are anisotropic with rising humidity. Namely $L_{200}$ increase from nearly $40 \AA$ to 47 $\AA$ and $L_{0 k 0}$ decrease from $47 \AA$ to $38 \AA$. This we attribute to the hydrophobic interactions along the $x$-axis and the hydrophilic interactions along the crystal's $y$-axes. 
Table 9.2: Values from linear regression of different crystallite parameters from immersed fibers. Linear regression was performed in an interval of 0.5-28\% strain for NM and 1-34\% strain for NC. $q_{h k l}$ peak position and $L_{h k l}$ crystallite length calculated from fwhm; aw $w_{120}$ angular width from arc slices, shown in figure 3.10c.

\begin{tabular}{|l|cl|cc|}
\hline \multicolumn{1}{|c|}{$\mathrm{x}$} & \multicolumn{2}{|c|}{ NC } & \multicolumn{2}{c|}{ NM } \\
& \multicolumn{2}{|c|}{ intercept } & slope & \multicolumn{2}{c|}{ intercept } & slope \\
\hline$q_{120}[\AA]^{-1}$ & 1.426 & $-6.74 E-5$ & 1.426 & $-1.03 E-4$ \\
$\operatorname{error}$ & $\pm 1.40 E-3$ & $\pm 1.08 E-4$ & $\pm 1.30 E-3$ & $\pm 1.48 E-4$ \\
$q_{200}[\AA]^{-1}$ & 1.183 & $-1.02 E-4$ & 1.176 & $-2.54 E-4$ \\
$\operatorname{error}$ & $\pm 1.84 E-3$ & $\pm 1.78 E-4$ & $\pm 3.74 E-3$ & $\pm 2.30 E-4$ \\
\hline$L_{120}[\AA]$ & 56.34 & -0.48 & 49.39 & -0.09 \\
$\operatorname{error}$ & \pm 1.61 & \pm 0.07 & \pm 2.51 & \pm 0.19 \\
$L_{200}[\AA]$ & 44.34 & 0.27 & 43.06 & 0.07 \\
error & \pm 2.06 & \pm 0.11 & \pm 0.59 & \pm 0.05 \\
\hline aw $w_{120}[\AA]$ & 26.48 & -0.08 & 26.94 & $1.3 E-3$ \\
error & \pm 0.56 & \pm 0.03 & \pm 0.03 & \pm 0.04 \\
\hline$d_{\text {amorph }}[\AA]$ & 3.843 & $-3.87 E-3$ & 3.843 & $-3.87 E-3$ \\
error & $\pm 3.74 E-3$ & $\pm 2.30 E-4$ & $\pm 1.47 E-2$ & $\pm 1.74 E-3$ \\
$\Delta q_{\text {amorph }}[\AA]$ & 1.475 & $-8.17 E-3$ & 1.624 & $-1.15 E-3$ \\
error & $\pm 7.78 E-2$ & $\pm 3.80 E-3$ & $\pm 1.19 E-2$ & $\pm 2.64 E-3$ \\
\hline
\end{tabular}




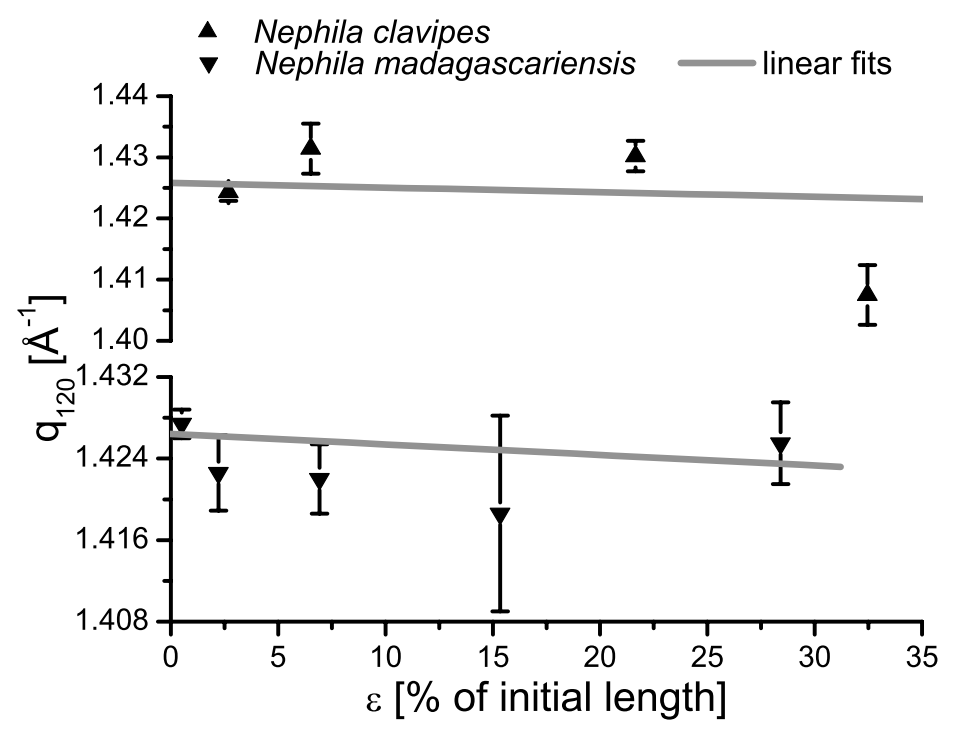

Figure 9.9: Tensile behavior of the equatorial (120)-peak position $q_{120}$ of immersed dragline from $\mathrm{N}$. clavipes and $\mathrm{N}$. madagascariensis. The changes by the applied strain are nearly negligible.

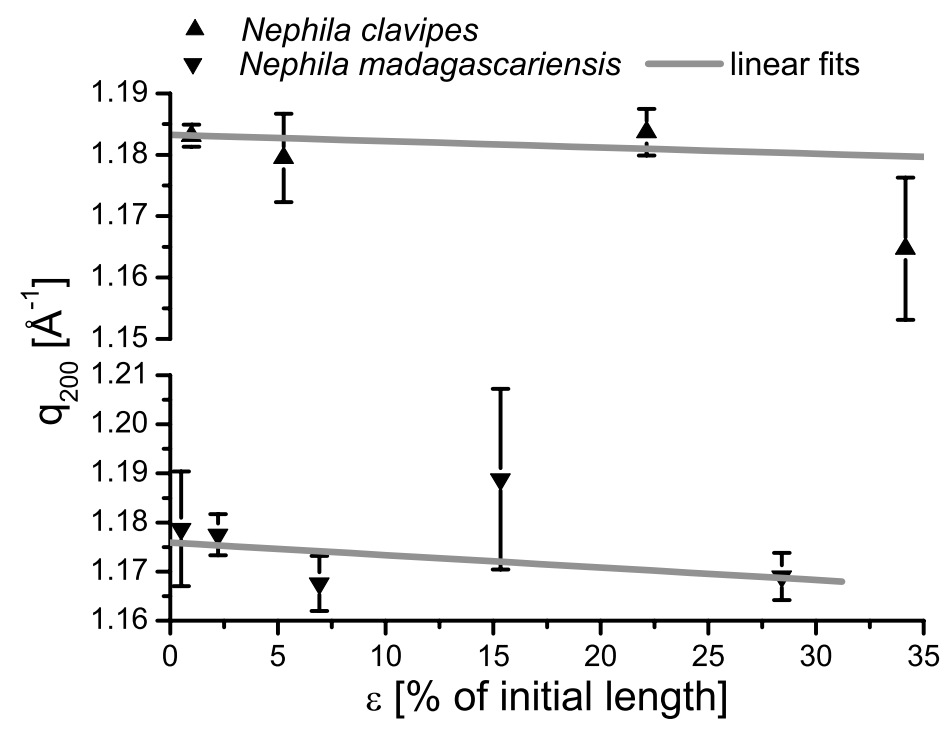

Figure 9.10: Tensile response of the equatorial peak position $q_{200}$ of immersed dragline to external strain. As for $q_{120}$ the changes are negligible. 


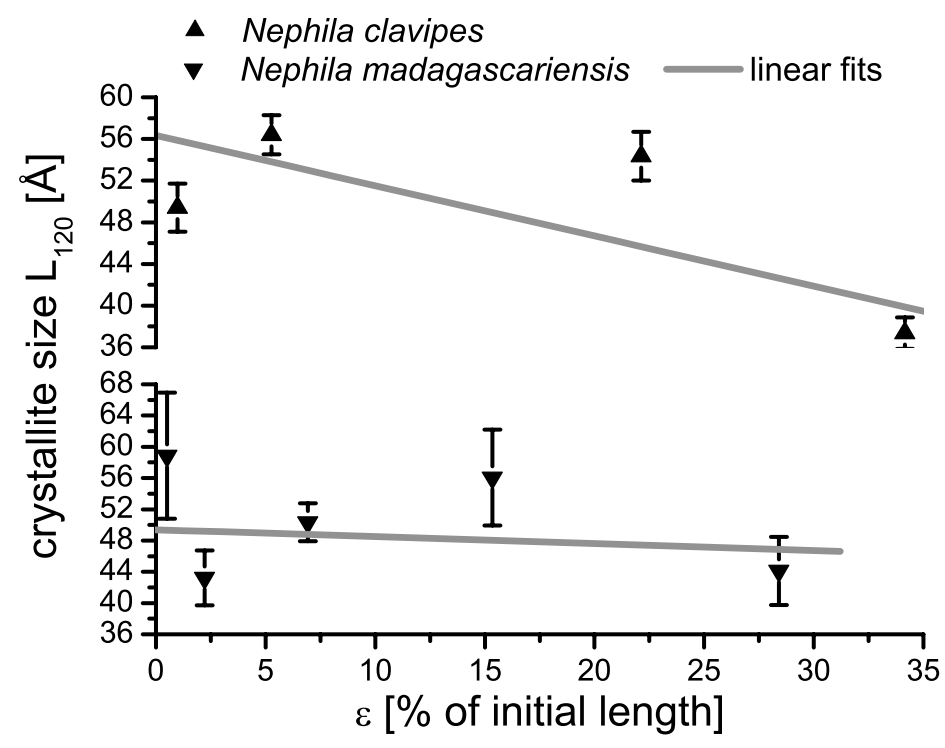

Figure 9.11: Tensile response of the crystallite size $L_{120}$ of immersed dragline to external strain. For both spider species the crystallite size decreases perpendicular to the direction of the applied strain.

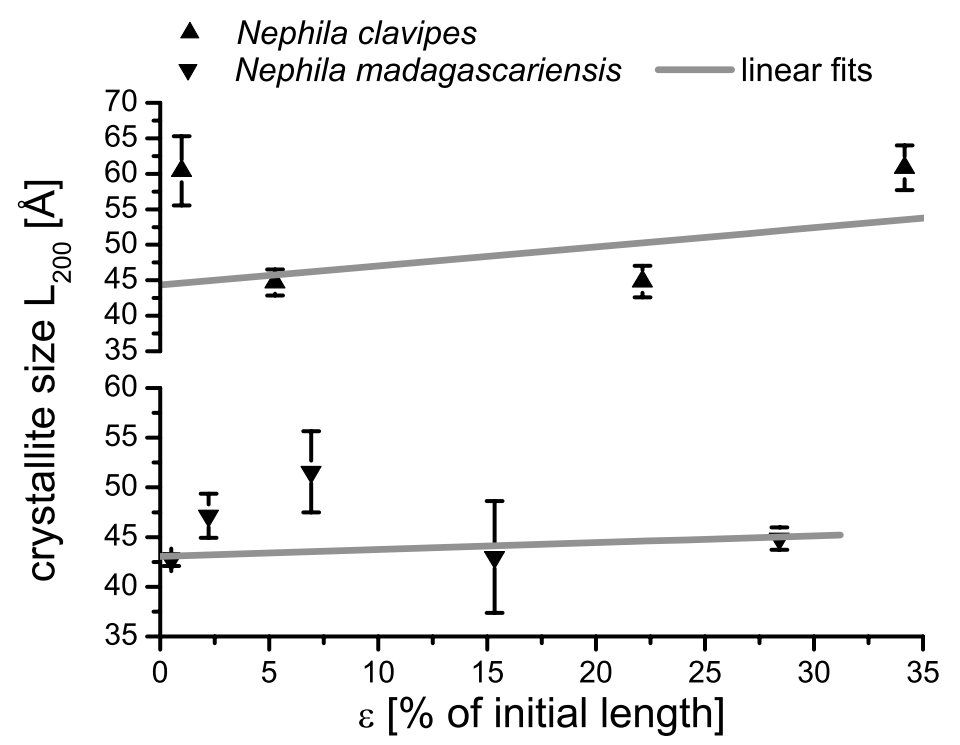

Figure 9.12: Response of the crystallite size $L_{200}$ of immersed dragline to external strain. The increase given by the linear regression for $\mathrm{N}$. clavipes is problematic due to the fact the $L_{200}$ is nearly the same for the begin and the end of the extension. 


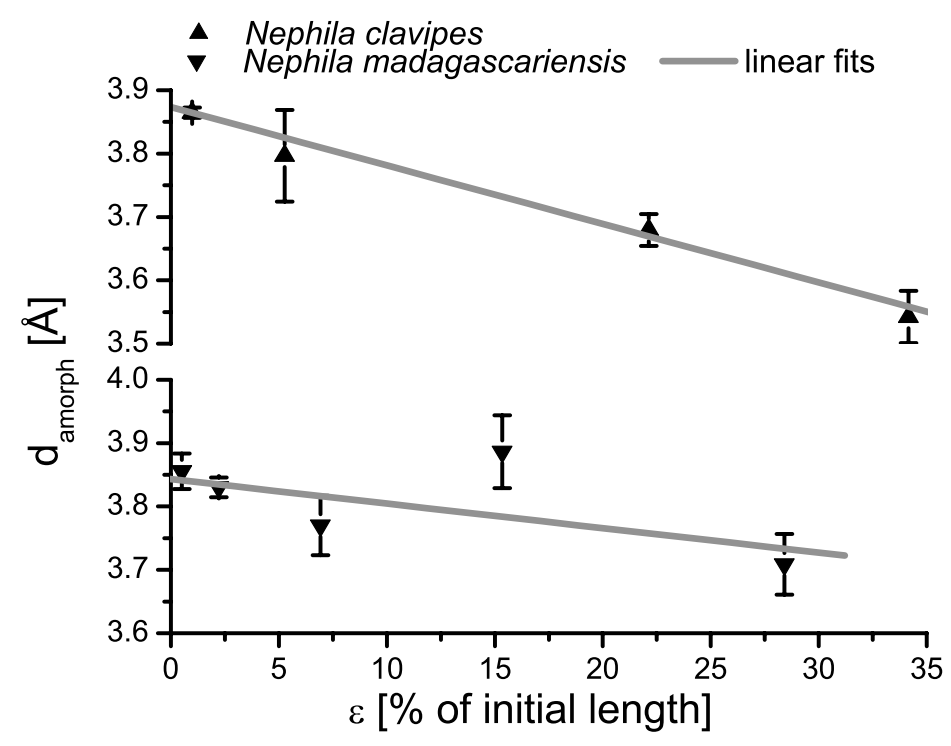

Figure 9.13: Mean distance $d_{\text {amorph }}$ of the amorphous matrix versus strain \& for immersed samples. It deceases for both species but much stronger for $\mathrm{N}$. clavipes.

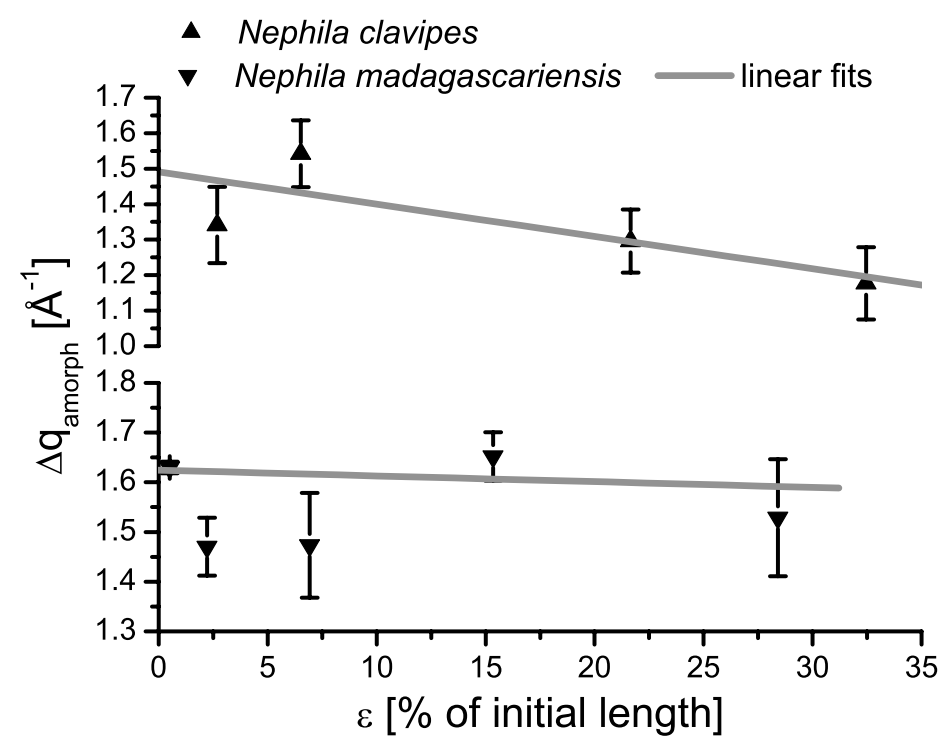

Figure 9.14: Width of the amorphous halo $\Delta d_{\text {amorph }}$ of the amorphous matrix versus strain $\varepsilon$ for immersed samples. For $\mathrm{N}$. clavipes it deceases significantly while it stays nearly the same for $\mathrm{N}$. madagascariensis. 


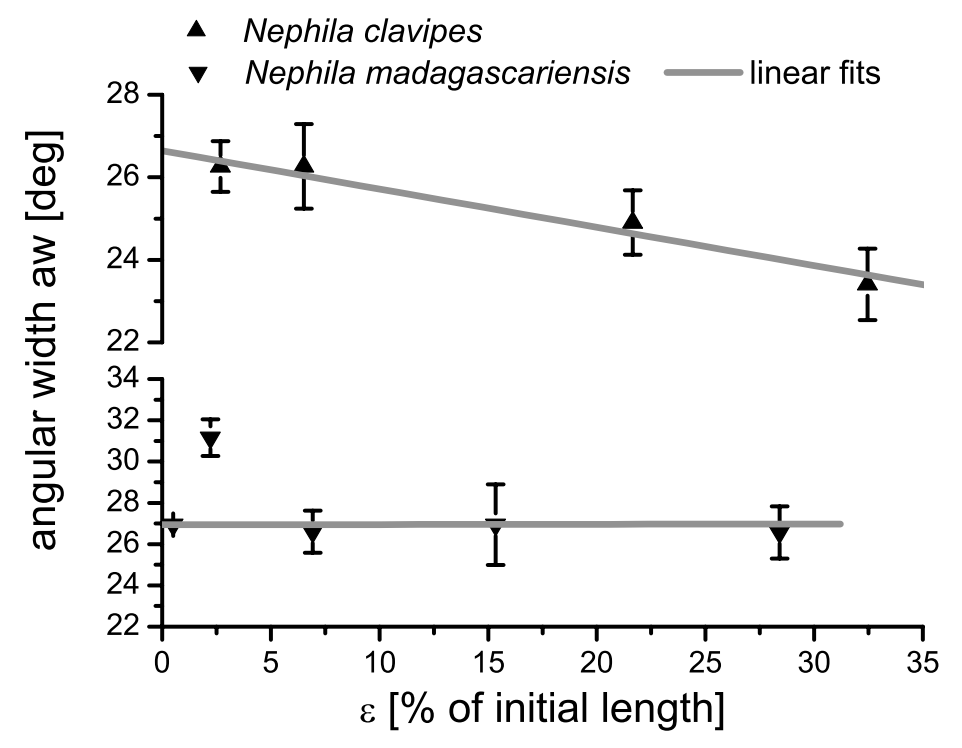

Figure 9.15: Response of the angular width aw $w_{120}$ of immersed samples on extension $\varepsilon$. For $\mathrm{N}$. clavipes it deceases significantly while it stays nearly the same for $\mathrm{N}$. madagascariensis. 


\section{Chapter 10}

\section{Summary}

In this study we have investigated the structural response of dragline silk to temperature, humidity and external strain.

We have focused on the structural properties of the crystalline components. Experimentally, we have first shown that strain- and humidity-induced structural changes can be monitored by single fiber X-ray scattering. The method is sensitive enough to observe the effect of strain on the lattice constants of the $\beta$-sheet crystallites, see figure $8.3 \mathrm{a}$. The observed response of the crystallite structure to the relative humidity as well as thermal and mechanical load were surprising. As mentioned in section 2.2, the crystalline components are often considered to be inactive cross links connecting different protein-strands and as a passive filler material reinforcing the amorphous matrix.

The temperature experiments, described in chapter 7 , indicate a surprising stability of the $\beta$-sheet crystallites against thermal denaturation. Up to $195^{\circ} \mathrm{C}$ the crystallite size was found to increase, and denaturation was not observed below $210^{\circ} \mathrm{C}$ as indicated by a decrease of the crystallite size. The initial growth of the crystallites with temperature is anisotropic in $x$ - and $y$-direction and accompanied by a change in composition. These phenomena correlate with the glass transition found in spider silk by DSC, which is mostly attributed to the amorphous matrix.

Under strain at ambient conditions, as well as under humid conditions, the crystallites align along the fiber axis in the direction of the force. Furthermore, the strain causes a radial broadening of the equatorial peaks which dominate the diffraction pattern. Such behavior can be interpreted as a 'splitting' or 'unfolding' of the crystallites. Accordingly, a number of hydrogen bonds in the crystallites need to be broken under mechanical load. A simple estimate shows that the number of broken bonds is sufficient to explain the amount of energy dissipation in the fiber. If this interpretation proves correct some assumptions about the origin of spider silk's mechanical properties had to be reconsidered.

In the model by $Y$. Termonia, presented in chapter 2.5 .2 , the $\beta$-sheets are regarded as passive elements which mainly contribute to the fibers stress-strain behavior by constraints of the amorphous strands emanating from the crystallites. It seems logical to incorporate our findings in such a model and to study the effect of a strain-dependent crystallite size on the network's tensile response. A generalized model could be validated by simulation of the X-ray scattering from the model 
network, as outlined in chapter 6, in particular if it can explain the unexpected behavior of the amorphous halo, see section 8.1.4 and 9.2.4. Most likely a generalized model with strain dependent crystallite properties, as the crystallite size, may also be able to reproduce the observed hysteresis behavior, as described in chapter 2.4 and 5 .

In addition, humidity induced changes in the diffraction pattern have been recorded, notably a broadening of the equatorial reflections. Accordingly, the lateral size of the crystallites (along the given direction in the lattice) $L_{120}$ decreases from approximately $54 \AA$ to $43 \AA$, while $L_{200}$ increases from nearly $40 \AA$ to 47 $\AA$ after immersion of a dry fiber in water. This can be interpreted in the light of recovery of the fiber's mechanical properties after immersion in water. A previously stretched fiber will show an original stress-strain behavior after immersion and drying. The fibers 'stretching history' is erased completely. If the crystallite size is connected to the stress-strain behavior, as discussed in the previous paragraph, one may interpret the recovery as reformation of broken bonds in the crystallites driven by the hydrophobicity of the alanine side chains.

Although some of the observed phenomena need further experimental and theoretical studies, we are confident that our findings will be helpful for a quantitative understanding of this fascinating biomaterial. 


\section{Abbreviations}

aa amino acid / amino acids

BM Bombyx mori silkworm which produces the silk for textiles

CRL Compound Refractive Lens, refractive optic for X-ray focusing

DSC Differential Scanning Calorimetry

ESRF European Synchrotron Radiation Facility

FTIR Fourier Transformed Infrared Spectroscopy

fwhm Full Width at Half Maximum

KB Kirkpatrick-Baez, mirror optic for X-ray focusing

HASYLAB Hamburger Synchrotronstrahlungslabor

MAS major ampullate silk, synoymous for dragline

NC Nephila clavipes, orb-web spider (Tetragnathidae) from Florida

NM Nephila madagascariensis, orb-web spider (Tetragnathidae) from East Africa

NMR Nuclear Magnetic Resonance

NS Nephila senegalensis, orb-web spider(Tetragnathidae) from West Africa

PE polyethylene

PP polypropylene

RH relative humidity 



\section{Bibliography}

[1] T. Scheibel. Mechanical properties of recombinant spider silk proteins. Applied Physics A, 82:191-192, 2006.

[2] D. Huemmerich, T. Scheibel, F. Vollrath, S. Cohen, U. Gat, and S. Ittah. Novel assembly properties of recombinant spider dragline silk protein. Current Biology., 14:2070-2074, 2006.

[3] S. Rammensee, D. Huemmerich, K. Hermanson, T. Scheibel, and A. Bausch. Rheological characterisation of recombinant spider silk nanofiber networks. Applied Physics A, 82:261-264, 2006.

[4] W. A. Shear, J. M. Palmer, J. A. Coddington, and P. M. Bonamo. A devonian spinneret: Early evidence of spiders and silk use. Science, 246:479-481, 1989.

[5] D. T. Grubb and L. W. Jelinski. Fiber morphology of spider silk: The effects of tensile deformation. Macromolecules, 30:2860-2867, 1997.

[6] Z. Yang, D. T. Grubb, and L. W. Jelinski. Small-angle x-ray scattering of spider dragline silk. Macromolecules, 30:8254-8261, 1997.

[7] C. Riekel and F. Vollrath. Spider silk fibre extrusion: combined wide- and small-angle x-ray microdiffraction experiments. International Journal of Biological Macromolecules, 29:203-210, 2001.

[8] C. Riekel, M. Mueller, and F. Vollrath. In situ x-ray diffraction during forced silking of spider silk. Macromolecules, 32:4464-4466, 1999.

[9] C. Riekel, C. I. Bränden, C. Craig, C. Ferrero, F. Heidelbach, and M. Müller. Aspects of x-ray diffraction on single spider fibers. International Journal of Biological Macromolecules, 24:179-186, 1999.

[10] A. Bram, C. I. Bränden, I. Snigireva C. Craig, and C. Riekel. X-ray diffraction from single fibers of spider silk. Journal of Applied Crystallography, 30:390-392, 1997.

[11] A. Glišović, J. Thieme, P. Guttmann, and T. Salditt. Transmission x-ray microscopy of spider dragline silk. International Journal of Biological Macromolecules, 40:87-95, 2007.

[12] A. Glišović. Röntgenstrukturanalyse von spinnenseide. Diplomarbeit, Universität des Saarlandes, 2004. 
[13] H. M. Peters. Über den spinnapperat von Nephila madagscariensis. Zeitschrift für Naturforschung, 10b:396-400, 1955.

[14] F. Vollrath. Spider webs and silks. Scientific American, March:52-54, 1992.

[15] P. D. Knight and F. Vollrath. Liquid crystalls and flow elongation in a spider's silk production line. Proceedings of the Royal Society B, 266:519 - 523, 1999.

[16] J. M. Kenney, D. Knight, M. J. Wise, and F. Vollrath. Amyloidogenic nature of spider silk. European Journal of Biochemistry, 269:4159-4163, 2002.

[17] H.-J. Jin and D. L. Kaplan. Mechanism of silk processing in insects and spiders. Nature, 424:1057-1061, 2003.

[18] F. Vollrath. Liquid crystalline spinning of spider silk. Nature, 410:541 - 548, 2001.

[19] C. Dicko, F. Vollrath, and J. M. Kenney. Spider silk protein refolding is controlled by changing ph. Biomacromolecules, 5:704-710, 2004.

[20] C. Dicko, J. M. Kenney, D. Knight, and F. Vollrath. Transition of a $\beta$-sheetrich structure in spidroin in vitro: The effect of ph and cations. Biochemistry, 43:14080-14087, 2004.

[21] Z. Shao and F. Vollrath. Materials: Surprising stength of silkworm silk. Nature, 418:741 - 741, 2002.

[22] K. B. Guess and C. Viney. Thermal analysis of major amullate (dragline) spider silk: the effect of spinning rate on tensile modulus. Thermochimica Acta, 315:61-66, 1998.

[23] Z. J. Pan, C. P. Li, and Q. Xu. Active control on molecular conformations and tensile properties of spider silk. Journal of Applied Polymer Science, 92:901-905, 2004.

[24] A. Sponner, E. Unger, F. Grosse, and K. Weisshart. Differential polymerization of the two main protein components of dragline silk during fibre spinning. Nature Materials, 4:772-775, 2005.

[25] R. F. Foelix. Biology of Spiders, chapter Spider Webs. Oxford University Press,, 2 edition, 1996.

[26] S. J. Lombardi and D. L. Kaplan. The amino acid composition of major ampullate gland silk (dragline) of Nephila clavipes (araneae, tetragnathidae). Journal of Arachnology, 18:297-306, 1990.

[27] A. Spooner, B. Schlott, F. Vollrath, E. Unger, F. Grosse, and K. Weisshart. Characterization of the protein components of Nephila clavipes dragline silk. Biochemistry, 44:4727-4736, 2005. 
[28] Swiss Institute of Bioinformatics. Expasy - expert protein analysis system. ExPASy homepage. http:/ / www.expasy.org.

[29] G. N. Ramachandran and V. Sasisekharan. Conformation of polypeptides and proteins. Advances in Protein Chemistry, 23:283-438, 1968.

[30] W. Hoppe et al. Biophysik, chapter 2.3.5. Springer-Verlag Berlin, 2 edition, 1982.

[31] M. Xu and R. V. Lewis. Structure of a protein superfiber: Spider dragline silk. Proceedings of the National Academy of Sciences of the United States of America (PNAS), 87:7120-7124, 1990.

[32] M. B. Hinman and R. V. Lewis. Insolation of a clone encoding a second dragline silk fibroin. Journal of Biological Chemistry, 267:19320-19324, 1992.

[33] J. Gatesy, C. Hayashi, D. Motruk, J. Woods, and R. V. Lewis. Extreme diversity, conservation and convergence of spider silk fibroin sequences. Science, 291:2603-2605, 2001.

[34] A. D. Parkhe, S. K. Seeley, K. Gardner, L. Thompson, and R. V. Lewis. Structural studies of spider silk proteins in the fiber. Journal of Molecular Recognition, 10:1-6, 1997.

[35] B. L. Thiel, K. B. Guess, and C. Viney. Non-periodic lattice crystals in the hierarchical microstructure of spider (major ampullate) silk. Biopolymers, 41:703719, 1997.

[36] J. D. van Beek, S. Hess, F. Vollrath, and B. H. Meier. The molecular structure of spider dragline silk: Folding and orientation of the protein backbone. Proceedings of the National Academy of Sciences of the United States of America (PNAS), 99:10226-10271, 2002.

[37] Z. Dong, R. V. Lewis, and C. R. Middaugh. Molecular mechanism of spider silk elasticity. Archives of Biochemistry and Biophysics, 284:53-57, 1991.

[38] D. Wilson, R. Valluzzi, and D. Kaplan. Conformational transition in model silk peptides. Biophysical Journal, 78:2690-2701, 2000.

[39] D. Porter, F. Vollrath, and Z. Shao. Predicting the mechanical properties of spider silk as a model nanostructured polymer. European Physical Journal E, 16:199 - 206, 2005.

[40] J. M. Gosline, M. W. Denny, and M. E. DeMont. Spider silk as rubber. Nature, 309:551-552, 1984.

[41] R. E. Marsh, R. B. Corey, and L. Pauling. An investigation of the structure of silk fibroin. Biochimica et Biophysica Acta, 16:1-34, 1955.

[42] J. O. Warwicker. Comparative studies of fibroins - ii. the crystal structures of various fibroins. Journal of Molecular Biology, 2:350-362, 1960. 
[43] A. Glišović and T. Salditt. Temperature dependent structure of spider silk by x-ray diffraction. Applied Physics A, January 2007. published online.

[44] F. N. Braun and C. Viney. Modelling self assembly of natural silk solutions. International Journal of Biological Macromolecules, 32:59-65, 2003.

[45] A. H. Simmons, C. A. Michal, and L. W. Jelinski. Molecular orientation and two-component nature of the crystalline fraction of spider dragline silk. Science, 271:84-87, 1996.

[46] E. Oroudjev, J. Soares, S. Arcidiancono, J. B. Thompson, S. A. Fossey, and H. G. Hansma. Segmented nanofibers of spider dragline silk: Atomic force microscopy and single-molecule force spectroscopy. Proceedings of the $\mathrm{Na}$ tional Academy of Sciences of the United States of America (PNAS), 99:6460-6465, 2002.

[47] R. Valluzzi and H.-J. Jin. X-ray evidence for a 'super'-secondary structure in silk fibers. Biomacromolecules, 5:696-703, 2004.

[48] D. Sapede, T. Seydel, V. T. Forsyth, M. M. Koza, R. Schweins, F. Vollrath, and C. Riekel. Nanofibrillar structure and molecular mobility in spider dragline silk. Macromolecules, 38:8447-8453, 2005.

[49] K. Augsten, P. Mühlig, and C. Herrmann. Glycoprotein and skin-core structure in Nephila clavipes spider silk observed by light and electron microscopy. Scanning, 22:12-15, 2000.

[50] S. Putthanarat, N. Stribeck, S. A. Fossey, R. K. Eby, and W. W. Adams. Investigation of the nanofibrils of silk fibers. Polymer, 41:7735 Đ7747, 2000.

[51] G. Zubay. Biochemistry. McGraw-Hill, 4th edition edition, 1999.

[52] T. Vehoff, A. Glišović, H. Schollmeyer, and T. Salditt. Mechanical properties of spider dragline silk: Humidity, hysteresis and relaxation. submitted to Biopolymers, September 2006.

[53] T. Vehoff. Elasticity of spider dragline silk. Diplomarbeit, Georg-AugustUniversität Göttingen, 2006.

[54] F. I. Bell, I. J. McEwen, and C. Viney. Supercontractive stress in wet spider dragline. Nature, 416:37-37, 2002.

[55] R. W. Work. A comparative study of the supercontraction of major ampullate silk fibers of orb-web-building spiders (Araneae). Journal of Arachnology, 2:299-308, 1981.

[56] Y. Liu, Z. Shao, and F. Vollrath. Relationships between supercontraction and mechanical properties of spider silk. Nature Materials, 4:901-905, 2005.

[57] M. Elices, J. Pérez Rigueiro, G. Plaza, and G. V. Guinea. Recovery in spider silk fibers. Journal of Applied Polymer Science, 92:3537-3541, 2004. 
[58] J. M. Gosline, P. A. Guerette, C. S. Ortlepp, and K. N. Savage. Mechanical design of spider silks: From fibroin sequence to mechanicl function. The Journal of Experimental Biology, 202:3295-3303, 1999.

[59] S. A. Fossey and D. L. Kaplan. Polymer Data Handbook, chapter Silk Polymers. Oxford University Press,, 1 edition, 1999.

[60] C. Y. Hayashi, N. H. Shipley, and R. V. Lewis. Hypothesis that corralate the sequence, structure, and mechanical properties of spider silk proteins. International Journal of Biological Macromolecules, 24:271-275, 1999.

[61] L. Dai, Y. Zhang, and Z. Ou-Yang. Elastic theory of single spider silk protein molecule. Thin Solid Films, 438-439:382-385, 2003.

[62] S. A. Fossey and S. Tripathy. Atomistic modeling of interphases in spider silk fibers. International Journal of Biological Macromolecules, 24:119-125, 1999.

[63] H. Zhou and Y. Zhang. Hierarchical chain model of spider capture silk elasticity. Physical Review Letters, 94:028104, 2005.

[64] N. Becker, E. Oroudjev, S. Mutz, J. P. Cleveland, P. L. Hansma, C. Y. Hayashi, D. E. Makarov, and H. G. Hansma. Molecular nanosprings in spider capturesilk threads. Nature Materials, 2:278 - 283, 2003.

[65] Y. Termonia. Molecular modeling of spider silk elasticity. Macromolecules, 27:7378-7381, 1994.

[66] J. P. OBrien and KC. H. Gardner S. R. Fahnestock, Y. Termonia. Nylons from nature: Synthetic analogs to spider silk. Advanced Materials, 10:1185-1195, 1998.

[67] E. Guth. Theory of filler reinforcement. Journal of Applied Physics, 16:20-25, 1945.

[68] J. A. Marqusee. Chain conformation in lamellar semicrystalline interphases. Macromolecules, 19:2420-2426, 1986.

[69] Y. Termonia. Chain conformation at semicrystalline interphases. Macromolecules, 28:7667-7670, 1995.

[70] M. von Laue. Röntgenstrahlinterferenzen. Physikalische Zeitschrift, 14:1075$1079,1913$.

[71] C. Kittel. Einführung in die Festkörperphysik. Oldenburg-Verlag, 7. aufl. edition, 1988.

[72] W. H. Bragg and W. L. Bragg. The reflection of x-rays by crystals. Proceedings of the Royal Society A, 88:428-438, 1913.

[73] J. A. Nielsen and D. McMorrow:. Elements of Modern X-Ray Physics. John Wiley and Sons, 1st edition, 2001. 
[74] C. Riekel, A. Cedola, F. Heidelbach, and K. Wagner. Microdiffraction experiments on single polymeric fibers by synchrotron radiation. Macromolecules, 30:1033-1037, 1997.

[75] S. Roth, M. Burghammer, A. Janotta, and C. Riekel. Rotational disorder in poly(p-phenylene terephthalamide) fibers by x-ray diffraction with a $100 \mathrm{~nm}$ beam. Macromolecules, 36:1585-1593, 2003.

[76] C. Li. Strukturanalyse von antibiotischen Peptiden in Lipidmembranen mittels Röntgenreflektivität. PhD thesis, Georg-August-Universiät zu Göttingen, 2004.

[77] C. Riekel and R. J. Davies. Applications of synchrotron radiation micro-focus techniques to the study of polymer and biopolymer fibers. Current Opinion in Colloids E Interface Science, 9:396-403, 2005.

[78] B. Lengeler, C. G. Schroer, M. Kuhlmann, B. Benner, F. Gunzler, O. Kurapova, F. Zontone, A. Snigirev, and I. Snigirev. Refractive x-rays lenses. Journal of Physics D, 38:A218-A222, 2005.

[79] Dr. A. Hammersley. Fit2d. European Synchrotron Radiation Facility homepage. http://www.esrf.fr/computing/scientific/FIT2D/.

[80] R. W. Work and P. D. Emerson. An apparatus and technique for the forcible silking of spiders. Journal of Arachnology, 10:1-10, 1982.

[81] S. Michielsen. Effekt of moisture and orientation on the fracture of nylon 6,6 fibers. Journal of Applied Polymer Science, 52:1081-1089, 1994.

[82] R. Brill. Beziehung zwischen wasserstoffbindungen und eingien eigenschaften von polyamiden. Die Makromoleculare Chemie, 18:294-309, 1956.

[83] F. Ko, S. Kawabata, M. Inoue, M. Niwa, S. Fossey, and J. Song. Engineering properties of spider silk. Materials Research Society Symposium Proceedings, 2002.

[84] M. H. Meling. Modellierung und strukturanalyse von $\beta$-faltblattkristalliten in spinnenseide. Diplomarbeit, Max-Planck-Institut für Biophysikalische Chemie Göttingen, 2006.

[85] S. Ulrich, M. H. Meling, A. Glišović, T. Salditt, and A. Zippelius. Simulating the diffraction from $\beta$-sheets. to be published, 2006.

[86] C. H. Bamford, L. Brown, A. Elliott, W. E. Hanby, and I. F. Trotter. Alphaand beta-forms of poly-1-alanine. Nature, 173:27-29, 1954.

[87] D. Sapede. Contributions à la compráhension de la structure et de la dynamique hiérarchiques du fil de traîne de l'araignée. Doctorat, Universite Joseph FourierGrenoble I, 2006. 
[88] C. L. Craig, C. Riekel, M. E. Herberstein, R. S. Weber, D. Kaplan, and N. E. Pierce. Evidence for diet effects on the composition of silk proteins produced by spiders. Molecular Biology and Evolution, 17:1904-1913, 2000.

[89] Gromacs.org. Gromacs. http://www.gromacs.org.

[90] YASARA Biosciences. Yasara. http://www.yasara.org.

[91] S. Arnott, S. D. Dover, and A. Elliott. Structure of $\beta$-poly-l-alanine: Refined atomic co-ordinates for an anti-parallel beta-pleated sheet. Journal of Molecular Biology, 30:201-208, 1967.

[92] P. M. Cunniff, S. A. Fossey, M. A. Auerbach, J. W. Song, D. L. Kaplan, W. W. Adams, R. K. Eby, D. Mahoney, and D. L. Vezie. Mechanical and thermal properties of dragline silk from the spider nephila clavipes. Polymers for Advanced Technologies, 5:401-410, 1994.

[93] Y. Baskin and L. Meyer. Lattice constants of graphite at low temperatures. Physical Review, 100:544-544, 1955.

[94] B. D. Cullity. Elements of X-Ray Diffraction. Addison-Wesley Publishing Company, 2nd edition, 1978.

[95] M. L. Mansfield. Temperature-dependent changes in the structure of the amorphous domains of semicrystalline polymers. Macromolecules, 20:1384$1393,1987$.

[96] H. S. Sheu. Lattice deformation and thermal stability of crystals in spider silk. International Journal of Biological Macromolecules, 34:325-331, 2004.

[97] P. Kirkpatrick and A. V. Baez. Formation of optical images by x-rays. Journal of the Optical Society of America, 38:766-774, 1948.

[98] P. B. Bowden and R. J. Young. Deformation mechanisms in crystalline polymers. Journal of Material Science, 9:2034-2051, 1974.

[99] W. F. Reichert, D. Göritz, and E. J. Duschl. The double network, a model describing filled elastomers. Polymer, 34:1216-1221, 1993. 



\section{Appendix A}

\section{Small Angle X-Ray Scattering (SAXS) of Spider Silk}

In contrast to WAXS measurements, which give information of unit cell dimensions and crystallite size, with SAXS measurements correlations on higher length scale are observed and hold information about the crystals arrangement. The experiments were performed at the X33 beamline, EMBL outstation Hamburg. The beamline $\mathrm{X} 33$ is a bending magnet beamline for biological small-angle scattering on the DORIS Fan D, located in the HASYLAB (Hamburg, Germany). It uses a fixed wavelength of $1.5 \AA$ and is equipped with a MAR Image Plate detector [345 $\mathrm{mm}$ ]. Monochromaticity is achieved by an asymmetric cut Si [111] crystal. The beamline is optimised for low background data collection from macromolecular solutions by the use of a vacuum flytube which provided a sample-to-detector distance of $2800 \mathrm{~mm}$. The experiments were performed on fiber bundles and calibrated with the beamline's collagen standard (periodicity $64 \mathrm{~nm}$ ), see figure A.1. Due to the fact that the length scales observed are now in the range of some dozen nanometers the scattering is no longer ruled by the atomic arrangement but by the sample's electron density distribution. For Nephila dragline the density of the whole fiber is approximately $1.35 \mathrm{~g} / \mathrm{cm}^{3}$ [59] while the density of the crystalline fraction is approximately $1.36 \mathrm{~g} / \mathrm{cm}^{3}$ (calculated for 8 alanine amino acids per unit cell). So density and electron density contrast between the amorphous and the crystalline fraction are too low for a sufficient SAXS signal without further preparation, see figure A.2. The required electron density contrast was installed by taking advantage of the amorphous matrix's property to absorb water. The water permeates predominately into the amorphous parts but not into the crystalline.

After wetting it was possible to obtain a signal parallel to the fiber axis. The intensity profile of the arid sample was used for background correction and subtracted from the signal of the wetted sample. The peak was found to be around $0.085 \AA^{-1}$ and corresponds to a mean distance between the crystallites of $75 \AA$, which is in good agreements with the findings of other groups [7;46;48]. Perpendicular to the fiber axis it was impossible to measure a signal for both wet and dry state. The crystallite's interspacing was calculated with an assumed mean crystallite of 50 $\AA \times 50 \AA \times 60 \AA$ and a crystalline fraction of $20 \%$ to $30 \%$, see section 2.2 . The vol- 
ume $V$, the crystallite represents $20 \%$ to $30 \%$ of, shall have a square base with an edge length $\zeta$ and a height of $75 \AA$. So $\zeta$ can be found to be $45 \AA$ by the following calculation

$$
\begin{aligned}
\zeta_{30 \%}^{2} \times 75 \AA & =V=\frac{1}{0.33}\left[(50 \AA)^{2} \times 60 \AA\right] \\
\zeta & =\sqrt{\frac{V}{75 \AA}} \approx 77 \AA, \\
\zeta_{20 \%}^{2} \times 75 \AA & =V=\frac{1}{0.20}\left[(50 \AA)^{2} \times 60 \AA\right] \\
\zeta & =\sqrt{\frac{V}{75 \AA}} \approx 100 \AA .
\end{aligned}
$$

So the the distance in between adjacent $\beta$-sheets ranges from $30 \AA$ to $50 \AA$. It is remarkable that the three Nephila species only differ slightly in WAXS but show notable differences in SAXS. Only for Nephila senegalensis it was possible to achieve a sufficient density contrast for SAXS measurements after wetting with MilliQ water. Also the SAXS results published by other groups are from NS. This phenomena is not really understood yet but we anticipate that the slightly higher amount of hydrophilic amino acids in the amorphous matrix of NS is responsible for this finding. 


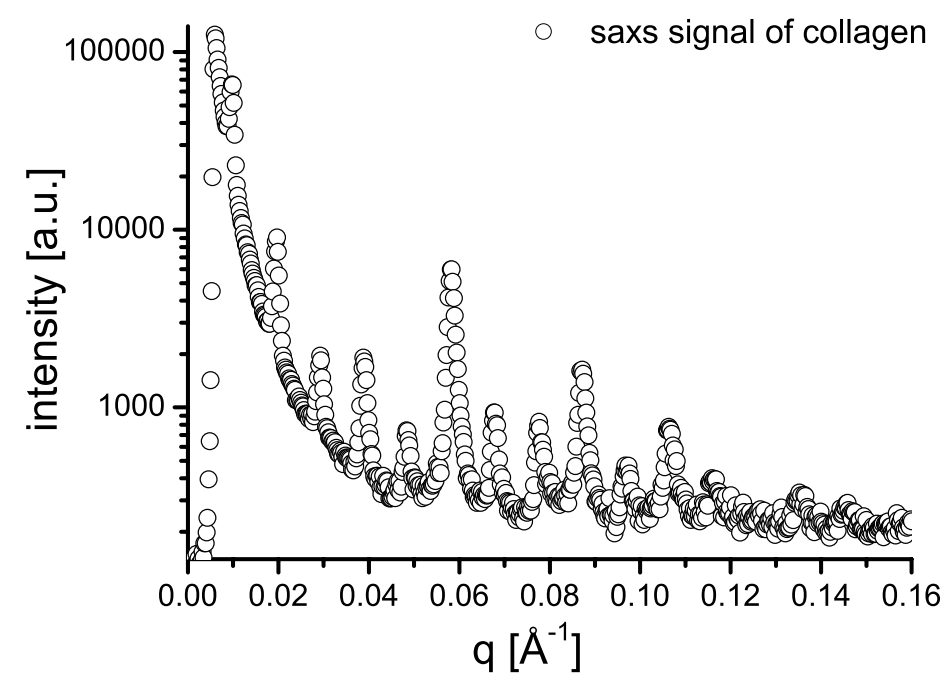

Figure A.1: 1D-SAXS signal of the collagen calibration sample available at the X33 small angle beamline at the EMBL outstation Hamburg. The periodicity is about $64 \AA$.

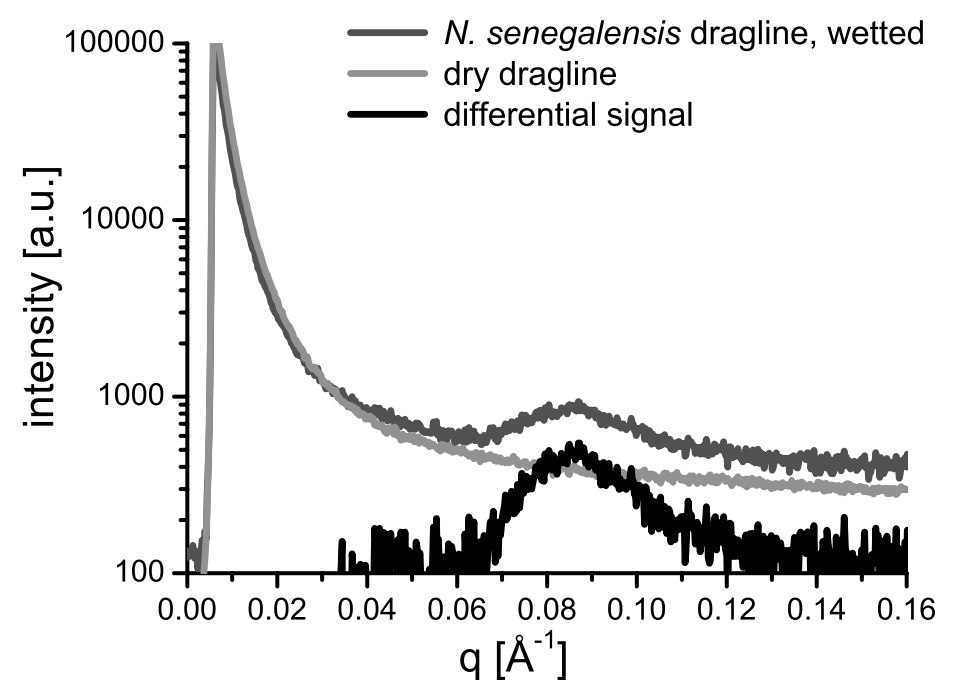

Figure A.2: 1D-SAXS signal of a NS fiber bundle. In the dry state the electron density contrast between amorphous and crystalline fraction is too low to obtain a signal. Only after wetting the contrast is strong enough. The profile of the dry sample was used for background correction. The maximum of the corrected curves lies around $0.085 \AA^{-1}$ which corresponds to a mean distance of $75 \AA$. 



\section{Appendix B}

\section{Primary Structures}

Experimental details regarding Nephilas' primary structures can be found in [31], [32] and [33].

\section{B.1 Nephila clavipes}

\section{Spidroin 1 (Fragment)}

\begin{tabular}{|c|c|c|c|c|}
\hline 10 & 20 & 30 & 40 & 50 \\
\hline QGAGAAAAAA & GGAGQGGYGG & LGGQGAGQGG & YGGLGGQGAG & QGAGAAAAAA \\
\hline 60 & 70 & 80 & 90 & 100 \\
\hline AGGAGQGGYG & GLGSQGAGRG & GQGAGAAAAA & AGGAGQGGYG & GLGSQGAGRG \\
\hline 110 & 120 & 130 & 140 & 150 \\
\hline GLGGQGAGAA & AAAAAGGAGQ & GGYGGLGNQG & AGRGGQGAAA & AAAGGAGQGG \\
\hline 160 & 170 & 180 & 190 & 200 \\
\hline YGGLGSQGAG & RGGLGGQGAG & AAAAAAGGAG & QGGYGGLGGQ & GAGQGGYGGL \\
\hline 210 & 220 & 230 & 240 & 250 \\
\hline GSQGAGRGGL & GGQGAGAAAA & AAAGGAGQGG & LGGQGAGQGA & GASAAAAGGA \\
\hline 260 & 270 & 280 & 290 & 300 \\
\hline GQGGYGGLGS & QGAGRGGEGA & GAAAAAAGGA & GQGGYGGLGG & QGAGQGGYGG \\
\hline 310 & 320 & 330 & 340 & 350 \\
\hline LGSQGAGRGG & LGGQGAGAAA & AGGAGQGGLG & GQGAGQGAGA & AAAAAGGAGQ \\
\hline 360 & 370 & 380 & 390 & 400 \\
\hline GGYGGLGSQG & AGRGGLGGQG & AGAVAAAAAG & GAGQGGYGGL & GSQGAGRGGQ \\
\hline$\overline{410}$ & $\widetilde{420}$ & 430 & 440 & 450 \\
\hline AGAAAAAAG & GAGQRGYGGL & GNQGAGRGGL & GGQGAGAAAA & AAAGGAGQGG \\
\hline 460 & 470 & 480 & 490 & 500 \\
\hline YGGLGNQGAG & RGGQGAAAAA & GGAGQGGYGG & LGSQGAGRGG & QGAGAAAAAA \\
\hline 510 & 520 & 530 & 540 & 550 \\
\hline VGAGQEGIRG & QGAGQGGYGG & LGSQGSGRGG & LGGQGAGAAA & AAAGGAGQGG \\
\hline 560 & 570 & 580 & 590 & 600 \\
\hline LGGQGAGQGA & GAAAAAAGGV & RQGGYGGLGS & QGAGRGGQGA & GAAAAAAGGA \\
\hline 610 & 620 & 630 & 640 & 650 \\
\hline GQGGYGGLGG & QGVGRGGLGG & QGAGAAAAGG & AGQGGYGGVG & SGASAASAAA \\
\hline 660 & 670 & 680 & 690 & 70 \\
\hline SRLSSPQASS & RVSSAVSNLV & ASGPTNSAAL & SSTISNVVSQ & IGASNPGLSC \\
\hline 710 & 720 & 730 & 740 & \\
\hline CDVLIQALLE & VVSALIQILG & SSSIGQVNYG & SAGQATQIVG & QSVYQAL \\
\hline
\end{tabular}




\section{Spidroin 2 (Fragment)}

$\begin{array}{rrrrr}10 & 20 & 30 & 40 & 50 \\ \text { PGGYGPGQQG } & \text { PGGYGPGQQG } & \text { PSGPGSAAAA } & \text { AAAAAAGPGG } & \text { YGPGQQGPGG } \\ 60 & 70 & 90 & 90 & 100 \\ \text { YGPGQQGPR } & \text { YGPGQQGPS } & \text { PGSAAAAAA } & \text { SGQQGPGGY } & \text { PRQQGPGGY } \\ 110 & 120 & 130 & 140 & 150 \\ \text { QGQQGPSGPG } & \text { SAAAASAAAS } & \text { AESGQQGPGG } & \text { YGPGQQGPGG } & \text { YGPGQQGPGG } \\ 160 & 170 & 180 & 190 & 200 \\ \text { YGPGQQGPG } & \text { PGSAAAAAAA } & \text { ASGPGQQGP } & \text { GYGPGQQGP } & \text { GYGPGQQGPS } \\ 210 & 220 & 230 & 240 & 250 \\ \text { GPGSAAAAAA } & \text { AASGPGQQGP } & \text { GGYGPGQQGP } & \text { GGYGPGQQGL } & \text { SGPGSAAAAA } \\ 260 & 270 & 280 & 290 & 300 \\ \text { AAGPGQQGPG } & \text { GYGPGQQGPS } & \text { GPGSAAAAAA } & \text { AAAGPGGYGP } & \text { GQQGPGGYGP } \\ 310 & 320 & 330 & 340 & 350 \\ \text { GQQGPSGAGS } & \text { AAAAAAAGPG } & \text { QQGLGGYGPG } & \text { QQGPGGYGPG } & \text { QQGPGGYGG } \\ 360 & 370 & 380 & 390 & 400 \\ \text { SASAAAAAAG } & \text { PGQQGPGGYG } & \text { PGQQGPSGPG } & \text { SASAAAAAAA } & \text { AGPGGYGPGQ } \\ 410 & 420 & 430 & 440 & 450 \\ \text { QGPGGYAPGQ } & \text { QGPSGPGSAS } & \text { AAAAAAAAGP } & \text { GGYGPGQQGP } & \text { GGYAPGQQGP } \\ 460 & 470 & 480 & 490 & 500 \\ \text { SGPGSAAAAA } & \text { AAAAGPGGYG } & \text { PAQQGPSGPG } & \text { IAASAASAGP } & \text { GGYGPAQQGP } \\ 510 & 520 & 530 & 540 & 550 \\ \text { AGYGPGSAVA } & \text { ASAGAGSAGY } & \text { GPGSQASAAA } & \text { SRLASPDSGA } & \text { RVASAVSNLV } \\ 560 & 570 & 580 & 590 & 600 \\ \text { SSGPTSSAAL } & \text { SSVISNAVSQ } & \text { IGASNPGLSG } & \text { CDVLIQALLE } & \text { IVSACVTILS } \\ 610 & 620 & & & \\ \text { SSSIGQVNYG } & \text { AASQFAQVVG } & \text { QSVLSAF } & & \end{array}$




\section{B.2 Nephila madagascariensis}

\section{Spidroin 1 (Fragment)}

\begin{tabular}{|c|c|c|c|c|}
\hline 10 & 20 & 30 & 40 & 50 \\
\hline GLGGQGAGQG & AGAAAAAAGG & AGQGGYGGLG & SQGAGRGGYG & GQGAGAAAAA \\
\hline & 70 & & & \\
\hline AAGGAGQGGY & GGLGSQGAGQ & GGYGGLGGQG & AGQGAAAAAA & AGGAGQGGYG \\
\hline 110 & 120 & 130 & 140 & 150 \\
\hline GLGSQGAGRG & GYGGQGAGAA & AAATGGAGQG & GYGGVGSGAS & AASAAASRLS \\
\hline 160 & 170 & 180 & 190 & \\
\hline SPQASSRVSS & AVSNLVASGP & TNSAALSSTI & SNAVSQIGAS & NPGLSGCDVL \\
\hline 210 & 220 & 230 & & \\
\hline IQALLEVVSA & LIHILGSSSI & GOVNYGSAGO & ATO & \\
\hline
\end{tabular}

\section{Spidroin 2 (Fragment)}

$\begin{array}{rrrrr}10 & 20 & 30 & 40 & 50 \\ \text { QGPSGPGSAA } & \text { AAAAAGPGQQ } & \text { GPGGYGPGQ } & \text { GPGGYGPGQQ } & \text { GPSGPGSAAA } \\ 60 & 70 & 90 & 100 \\ \text { AAAAAAAGPG } & \text { QQGPGGYGPG } & \text { PQGPGGYGP } & \text { QQGPSGYGPG } & \text { QQGPSGPGSA } \\ 110 & 120 & 130 & 140 & 150 \\ \text { ASAAAAAGG } & \text { QQGPGGYGPG } & \text { QQGPGGYGPG } & \text { QQGPSGPGSA } & \text { AAAAAAGPGQ } \\ 160 & 170 & 180 & 190 & 200 \\ \text { QGPGGYGPGQ } & \text { QGPGGYGQQ } & \text { QGPSGPGSAA } & \text { AAAAAAGPQ } & \text { QGPGGYGPGQ } \\ 210 & 220 & 240 & 250 \\ \text { QGPGGYGPGQ } & \text { QGPSGPGSAA } & \text { AAAAAGPGQQ } & \text { GPGGYGPQQ } & \text { GPGGYGPGQ } \\ 260 & 270 & 280 & 290 & 300 \\ \text { GPSGPGSAAA } & \text { AAAAAAGPGQ } & \text { QGPGGYGPGQ } & \text { QGPGQQGPSG } & \text { PGSAAAAAAA } \\ 310 & 320 & 330 & 340 & 350 \\ \text { GPGPQGPGY } & \text { GPGQQGPGGY } & \text { GPSGPGSAAA } & \text { AAAAAGPGQQ } & \text { GPGGYGPGQ } \\ 360 & 370 & 380 & 390 & 400 \\ \text { RPSGYGPGQQ } & \text { GPSGPGSAAA } & \text { AAAAGPGQG } & \text { PGAYGPSPG } & \text { SAAAAAGLGG } \\ 410 & 420 & 430 & 440 & 450 \\ \text { YGPAQQGPSG } & \text { AGSAAAAAAA } & \text { GPGGYGPVQQ } & \text { GPSGPGSAAG } & \text { PGGYGPAQQG } \\ 460 & 470 & 480 & 490 & 500 \\ \text { PARYGPGSA } & \text { AAAAAAGSAG } & \text { YGPGPQASAA } & \text { ASRLASPDSG } & \text { ARVASAVSNL } \\ 510 & 520 & 530 & 540 & 550 \\ \text { VSSGPTSSAA } & \text { LSSVISNAVS } & \text { QIGASNPGLS } & \text { GCDVLIQALL } & \text { EIVSACVTIL } \\ 560 & & & & \end{array}$

SSSSIGQVNY GAA 


\section{B.3 Nephila senegalensis}

\section{Spidroin 1 (Fragment)}

$\begin{array}{rrrrr}10 & 20 & 30 & 40 & 50 \\ \text { GLGGQGAGRG } & \text { AGAAAAAAGG } & \text { AGQGGYGGLG } & \text { GQGAGAAAAA } & \text { AGGAGQGGQG } \\ 60 & 70 & 80 & 90 & 100 \\ \text { LGGRGAAAAG } & \text { GAGQGGYGGL } & \text { GGQGAGRGAG } & \text { AAAAAAGGAG } & \text { QGGYGGLGGQ } \\ 110 & 120 & 130 & 140 & 150 \\ \text { GAGAAAAAAA } & \text { AGGAGQGGY } & \text { GLGSQGAGRG } & \text { GYGGQGAGAA } & \text { VAAIGGVGQ } \\ 160 & 170 & 180 & 190 & 200 \\ \text { GYGGVGSGAS } & \text { AASAAASRLS } & \text { SPEASSRVSS } & \text { AVSNLVSSGP } & \text { TNSAALSSTI } \\ 210 & 220 & 230 & 240 & 250 \\ \text { SNVVSQIGAS } & \text { NPGLSGCDVL } & \text { IQALLEVVSA } & \text { LVHILGSSSI } & \text { GQVNYGSAGQ }\end{array}$

ATQ

\section{Spidroin 2 (Fragment)}

$\begin{array}{rrrrr}10 & 20 & 30 & 40 & 50 \\ \text { QGPGGYGPSG } & \text { PGSAAAASAA } & \text { AGPGQQGPGA } & \text { YGPSGPGSAA } & \text { AAAGPGXYGP } \\ 60 & 70 & 80 & 90 & 100 \\ \text { GQQGPSGPGA } & \text { AAAAAGPGQ } & \text { GPGGYGPGAA } & \text { AAAAAAAGPG } & \text { QQGPVAYGPS } \\ 110 & 120 & 130 & 140 & 150 \\ \text { GPGSAASAAG } & \text { PGGYGPARYG } & \text { PSGSAAAAAA } & \text { AGAGSAGYGP } & \text { GPQASAAASR } \\ 160 & 170 & 180 & 190 & 200 \\ \text { LASPDSGARV } & \text { ASAVSNLVSS } & \text { GPTSSAALSS } & \text { VIXNAVSQIG } & \text { ASNPGLSGCD } \\ 210 & 220 & 230 & & \\ \text { VLIXALLEIV } & \text { SACVTILSSS } & \text { SIGQVNYGAA } & \text { ATQ } & \end{array}$




\section{Appendix C}

\section{Fit2D Macros}

The Fit2D macros to obtain one dimensional intensity profiles from 2D-diffraction patterns are given here. The Fit2D function 'Create Macro' was used to produce a macro for each slice type which then were combined. Fit2D is case sensitive, also the line breaks are essential. Slices require the definition of start pixel and end pixel, arc slices are per definition around the beam center and require start, middle and end pixel, for cake integrations inner radius, outer radius, start azimuth and end azimuth have to be defined by giving the associated pixel coordinates. The pixel size has to be given in $\mu \mathrm{m}$ and the sample to detector distance in $\mathrm{mm}$.

\section{C.1 Equatorial, Meridional and Arc Slices}

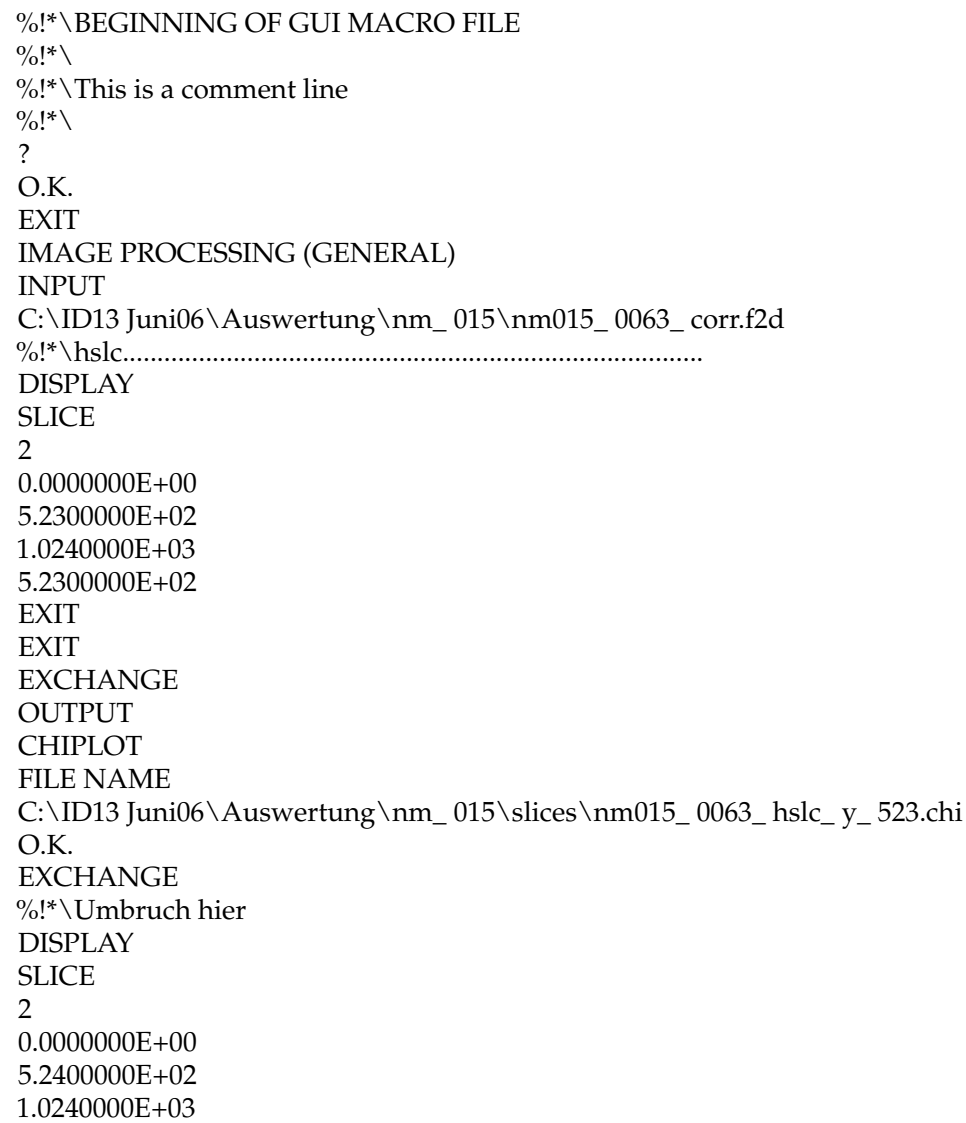




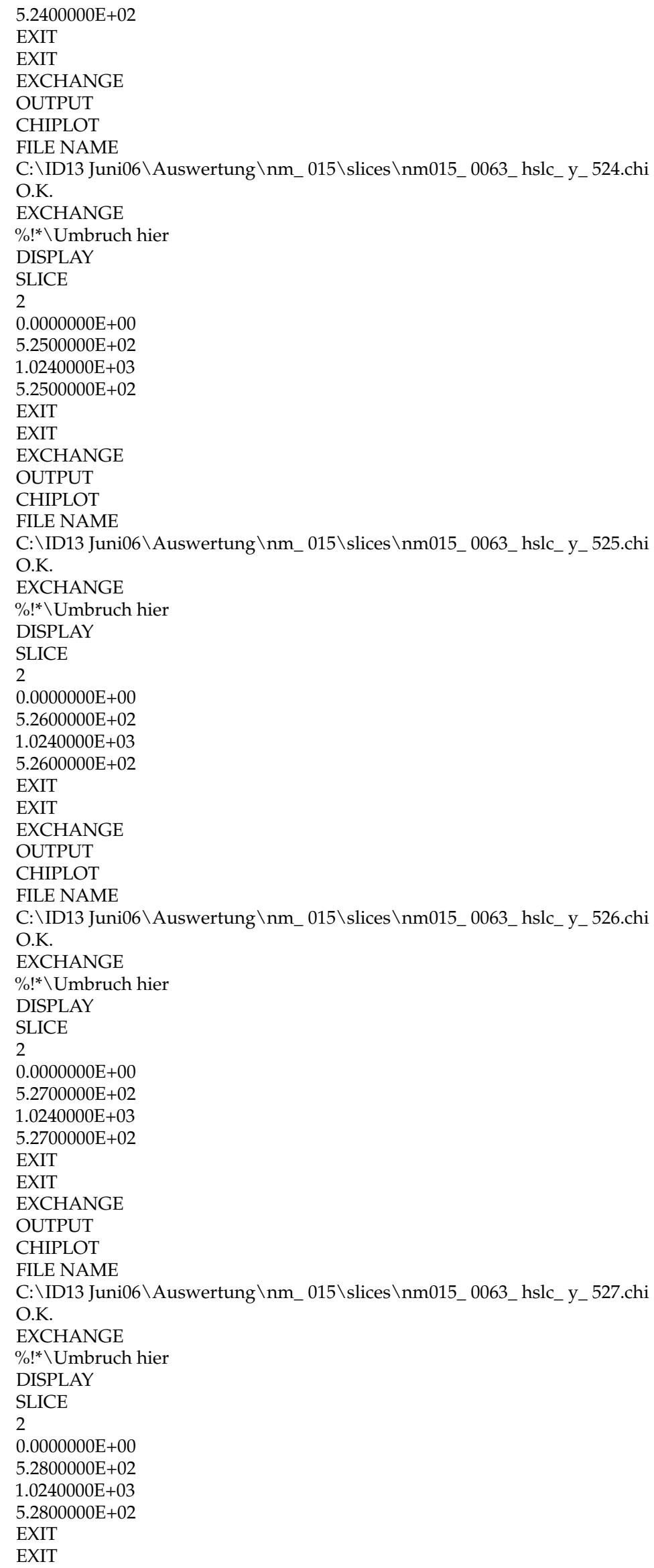




\section{EXCHANGE}

OUTPUT

CHIPLOT

FILE NAME

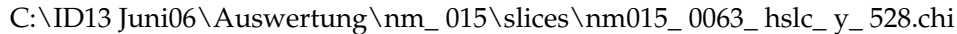

O.K.

EXCHANGE

$\% !^{*} \backslash$ Umbruch hier

DISPLAY

SLICE

2

$0.0000000 \mathrm{E}+00$

$5.2900000 \mathrm{E}+02$

$1.0240000 \mathrm{E}+03$

$5.2900000 \mathrm{E}+02$

EXIT

EXIT

EXCHANGE

OUTPUT

CHIPLOT

FILE NAME

C: \ID13 Juni06 \Auswertung $\backslash n m_{-}$015 \slices $\backslash$ nm015_0063_hslc_y_529.chi

O.K.

EXCHANGE

$\% !^{*} \backslash$ Umbruch hier

DISPLAY

SLICE

$0.0000000 \mathrm{E}+00$

$5.3000000 \mathrm{E}+02$

$1.0240000 \mathrm{E}+03$

$5.3000000 \mathrm{E}+02$

EXIT

EXIT

EXCHANGE

OUTPUT

CHIPLOT

FILE NAME

C: \ID13 Juni06 \Auswertung $\backslash n m_{-}$015 \slices $\backslash n m 015$ _0063_hslc_ y_530.chi

O.K.

EXCHANGE

$\% ! * \backslash$ Umbruch hier

DISPLAY

SLICE

2

$0.0000000 \mathrm{E}+00$

$5.3100000 \mathrm{E}+02$

$1.0240000 \mathrm{E}+03$

$5.3100000 \mathrm{E}+02$

EXIT

EXIT

EXCHANGE

OUTPUT

CHIPLOT

FILE NAME

C: \ID13 Juni06 \Auswertung \nm_015\slices $\backslash n$ m015_0063_hslc_y_531.chi

O.K.

EXCHANGE

$\% !^{*} \backslash$ Umbruch hier

DISPLAY

SLICE

2

$0.0000000 \mathrm{E}+00$

$5.3200000 \mathrm{E}+02$

$1.0240000 \mathrm{E}+03$

$5.3200000 \mathrm{E}+02$

EXIT

EXIT

EXCHANGE

OUTPUT

CHIPLOT 
FILE NAME

C: $\backslash$ ID13 Juni06 \Auswertung $\backslash n m_{-}$015 \slices $\backslash$ nm015_0063_hslc_y_532.chi O.K.

EXCHANGE

$\%$ !* $\backslash$ Umbruch hier

DISPLAY

SLICE

$0.0000000 \mathrm{E}+00$

$5.3300000 \mathrm{E}+02$

$1.0240000 \mathrm{E}+03$

$5.3300000 \mathrm{E}+02$

EXIT

EXIT

EXCHANGE

OUTPUT

CHIPLOT

FILE NAME

C: \ID13 Juni06 \Auswertung $\backslash n$ m_ 015 \slices $\backslash$ nm015_0063_hslc_y_533.chi

O.K.

EXCHANGE

$\%$ !* \vslc.......

DISPLAY

SLICE

2

$5.0800000 \mathrm{E}+02$

$0.0000000 \mathrm{E}+00$

$5.0800000 \mathrm{E}+02$

$1.0240000 \mathrm{E}+03$

EXIT

EXIT

EXCHANGE

OUTPUT

CHIPLOT

FILE NAME

C: \ID13 Juni06 \Auswertung $\backslash n m_{-}$015 \slices $\backslash n m 015 \_0063 \_$vslc_ X_ 508.chi

O.K.

EXCHANGE

$\% !^{*} \backslash$ Umbruch hier

DISPLAY

SLICE

$5.0900000 \mathrm{E}+02$

$0.0000000 \mathrm{E}+00$

$5.0900000 \mathrm{E}+02$

$1.0240000 \mathrm{E}+03$

EXIT

EXIT

EXCHANGE

OUTPUT

CHIPLOT

FILE NAME

C: \ID13 Juni06 \Auswertung $\backslash n m_{-}$015 \slices \nm015_0063_vslc_ X_ 509.chi

O.K.

EXCHANGE

$\%$ !* $\backslash$ Umbruch hier

DISPLAY

SLICE

2

$5.1000000 \mathrm{E}+02$

$0.0000000 \mathrm{E}+00$

$5.1000000 \mathrm{E}+02$

$1.0240000 \mathrm{E}+03$

EXIT

EXIT

EXCHANGE

OUTPUT

CHIPLOT

FILE NAME

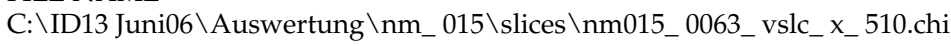

O.K. 
EXCHANGE

$\% ! * \backslash$ Umbruch hier

DISPLAY

SLICE

2

$5.1100000 \mathrm{E}+02$

$0.0000000 \mathrm{E}+00$

$5.1100000 \mathrm{E}+02$

$1.0240000 \mathrm{E}+03$

EXIT

EXIT

EXCHANGE

OUTPUT

CHIPLOT

FILE NAME

C: $\backslash$ ID13 Juni06 \Auswertung $\backslash n m_{-}$015 \slices $\backslash$ nm015_0063_vslc_ x_511.chi O.K.

EXCHANGE

$\% !^{*} \backslash$ Umbruch hier

DISPLAY

SLICE

2

$5.1200000 \mathrm{E}+02$

$0.0000000 \mathrm{E}+00$

$5.1200000 \mathrm{E}+02$

$1.0240000 \mathrm{E}+03$

EXIT

EXIT

EXCHANGE

OUTPUT

CHIPLOT

FILE NAME

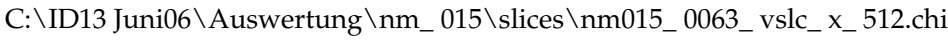
O.K.

EXCHANGE

$\% !^{*} \backslash$ Umbruch hier

DISPLAY

SLICE

$5.1300000 \mathrm{E}+02$

$0.0000000 \mathrm{E}+00$

$5.1300000 \mathrm{E}+02$

$1.0240000 \mathrm{E}+03$

EXIT

EXIT

EXCHANGE

OUTPUT

CHIPLOT

FILE NAME

C: $\backslash$ ID13 Juni06 \Auswertung $\backslash n$ m_ 015 \slices $\backslash$ nm015_0063_vslc_x_513.chi O.K.

EXCHANGE

$\%$ !* $\backslash$ Umbruch hier

DISPLAY

SLICE

2

$5.1400000 \mathrm{E}+02$

$0.0000000 \mathrm{E}+00$

$5.1400000 \mathrm{E}+02$

$1.0240000 \mathrm{E}+03$

EXIT

EXIT

EXCHANGE

OUTPUT

CHIPLOT

FILE NAME

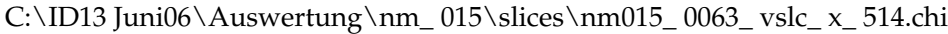

O.K.

EXCHANGE

$\% ! * \backslash$ Umbruch hier

DISPLAY 


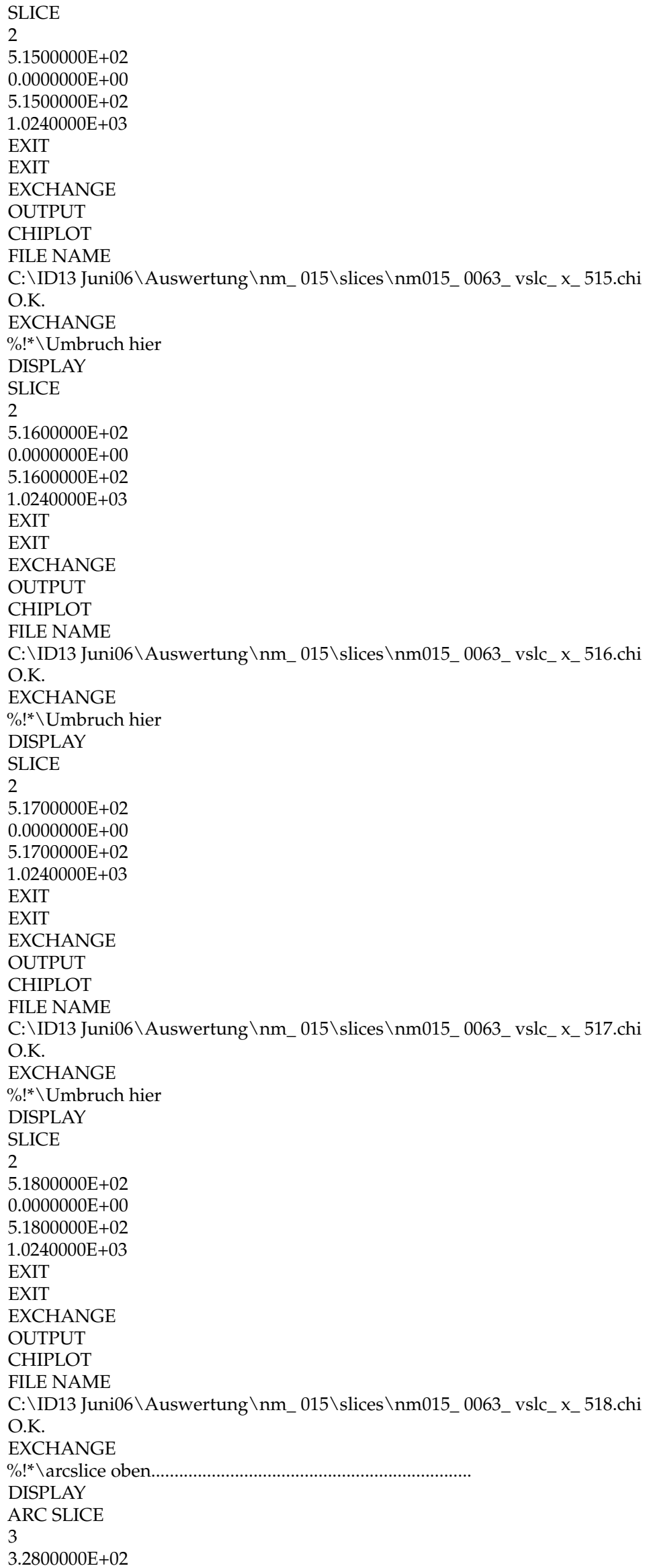


$5.2800000 \mathrm{E}+02$

$5.1300000 \mathrm{E}+02$

$7.0400000 \mathrm{E}+02$

$6.8000000 \mathrm{E}+02$

$5.2800000 \mathrm{E}+02$

EXIT

EXIT

EXCHANGE

OUTPUT

CHIPLOT

FILE NAME

C: \ID13 Juni06 \Auswertung \nm_015\slices \nm015_0063_aslc_y_704.chi

O.K.

EXCHANGE

$\%$ !* \Umbruch hier

DISPLAY

ARC SLICE

3

$3.2900000 \mathrm{E}+02$

$5.2800000 \mathrm{E}+02$

$5.1300000 \mathrm{E}+02$

$7.0300000 \mathrm{E}+02$

$6.7900000 \mathrm{E}+02$

$5.2800000 \mathrm{E}+02$

EXIT

EXIT

EXCHANGE

OUTPUT

CHIPLOT

FILE NAME

C: \ID13 Juni06 \Auswertung \nm_015 \slices $\backslash n m 015 \_0063 \_$aslc_y_703.chi O.K.

EXCHANGE

$\% ! * \backslash$ Umbruch hier

DISPLAY

ARC SLICE

3

$3.3000000 \mathrm{E}+02$

$5.2800000 \mathrm{E}+02$

$5.1300000 \mathrm{E}+02$

$7.0200000 \mathrm{E}+02$

$6.7800000 \mathrm{E}+02$

$5.2800000 \mathrm{E}+02$

EXIT

EXIT

EXCHANGE

OUTPUT

CHIPLOT

FILE NAME

C: \ID13 Juni06 \Auswertung $\backslash n$ m_ 015 \slices $\backslash$ nm015_0063_aslc_y_702.chi O.K.

EXCHANGE

$\%$ !* \Umbruch hier

DISPLAY

ARC SLICE

$3.3100000 \mathrm{E}+02$

$5.2800000 \mathrm{E}+02$

$5.1300000 \mathrm{E}+02$

$7.0100000 \mathrm{E}+02$

$6.7700000 \mathrm{E}+02$

$5.2800000 \mathrm{E}+02$

EXIT

EXIT

EXCHANGE

OUTPUT

CHIPLOT

FILE NAME

C: \ID13 Juni06 \Auswertung \nm_015\slices \nm015_0063_aslc_y_701.chi

O.K.

EXCHANGE 


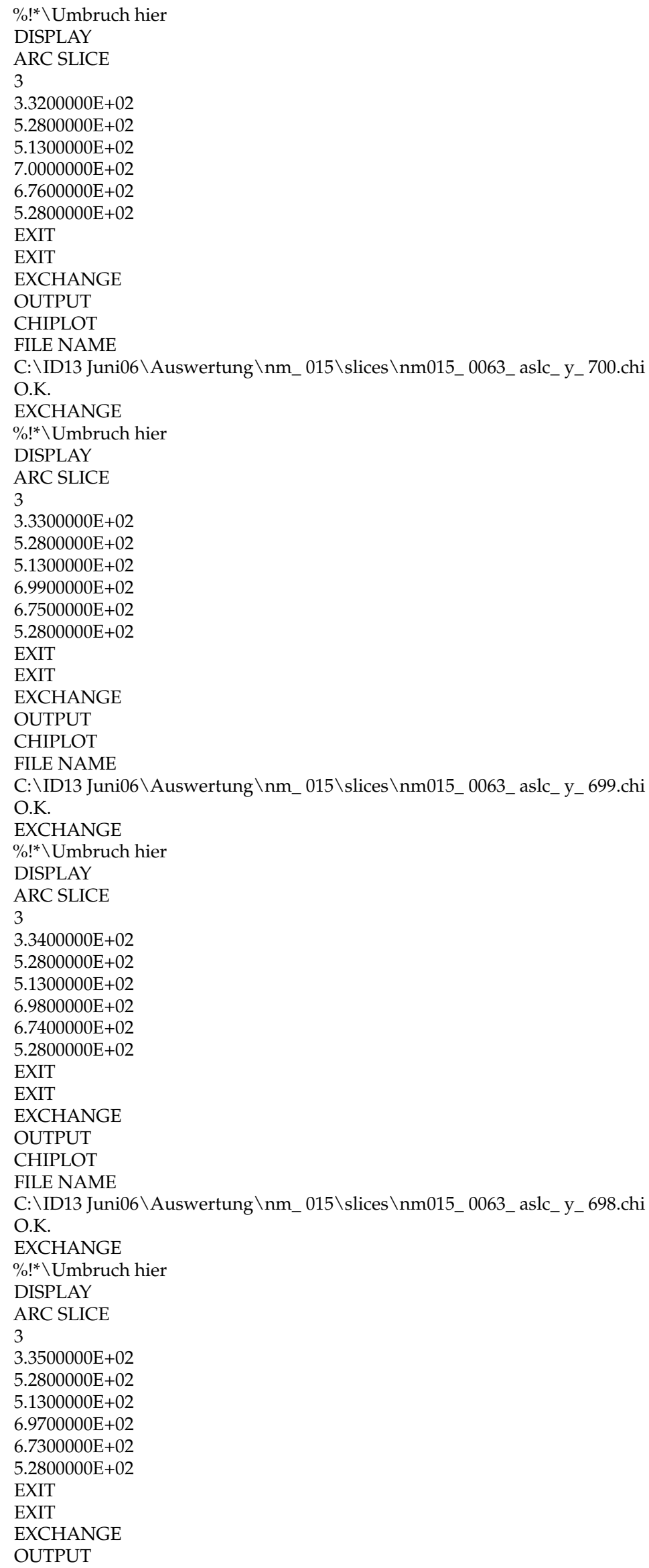


CHIPLOT

FILE NAME

C: \ID13 Juni06 \Auswertung \nm_015 \slices \nm015_0063_aslc_y_697.chi

O.K.

EXCHANGE

$\%$ !* \Umbruch hier

DISPLAY

ARC SLICE

3

$3.3600000 \mathrm{E}+02$

$5.2800000 \mathrm{E}+02$

$5.1300000 \mathrm{E}+02$

$6.9600000 \mathrm{E}+02$

$6.7200000 \mathrm{E}+02$

$5.2800000 \mathrm{E}+02$

EXIT

EXIT

EXCHANGE

OUTPUT

CHIPLOT

FILE NAME

C: \ID13 Juni06 \Auswertung \nm_015 \slices $\backslash n m 015 \_0063 \_$aslc_y_696.chi

O.K.

EXCHANGE

$\% !^{*} \backslash$ Umbruch hier

DISPLAY

ARC SLICE

3

$3.3700000 \mathrm{E}+02$

$5.2800000 \mathrm{E}+02$

$5.1300000 \mathrm{E}+02$

$6.9500000 \mathrm{E}+02$

$6.7100000 \mathrm{E}+02$

$5.2800000 \mathrm{E}+02$

EXIT

EXIT

EXCHANGE

OUTPUT

CHIPLOT

FILE NAME

C: $\backslash$ ID13 Juni06 \Auswertung $\backslash n m_{-}$015 \slices $\backslash n m 015 \_0063$ _aslc_y_ 695.chi O.K.

EXCHANGE

$\% !^{*} \backslash$ Umbruch hier

DISPLAY

ARC SLICE

3

$3.3800000 \mathrm{E}+02$

$5.2800000 \mathrm{E}+02$

$5.1300000 \mathrm{E}+02$

$6.9400000 \mathrm{E}+02$

$6.7000000 \mathrm{E}+02$

$5.2800000 \mathrm{E}+02$

EXIT

EXIT

EXCHANGE

OUTPUT

CHIPLOT

FILE NAME

C: \ID13 Juni06 \Auswertung \nm_015\slices \nm015_0063_aslc_y_694.chi

O.K.

EXCHANGE

$\% ! * \backslash$ Umbruch hier

$\% !^{*} \backslash$ arcslice unten.

DISPLAY

ARC SLICE

3

$3.2800000 \mathrm{E}+02$

$5.2800000 \mathrm{E}+02$

$5.1300000 \mathrm{E}+02$

$3.5200000 \mathrm{E}+02$ 
$6.8000000 \mathrm{E}+02$

$5.2800000 \mathrm{E}+02$

EXIT

EXIT

EXCHANGE

OUTPUT

CHIPLOT

FILE NAME

C: $\backslash$ ID13 Juni06 \Auswertung $\backslash n m_{-}$015 \slices $\backslash$ nm015_0063_aslc_y_ 352.chi O.K.

EXCHANGE

$\% !^{*} \backslash$ Umbruch hier

DISPLAY

ARC SLICE

3

$3.2900000 \mathrm{E}+02$

$5.2800000 \mathrm{E}+02$

$5.1300000 \mathrm{E}+02$

$3.5300000 \mathrm{E}+02$

$6.7900000 \mathrm{E}+02$

$5.2800000 \mathrm{E}+02$

EXIT

EXIT

EXCHANGE

OUTPUT

CHIPLOT

FILE NAME

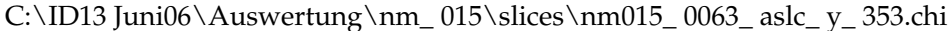

O.K.

EXCHANGE

$\% ! * \backslash$ Umbruch hier

DISPLAY

ARC SLICE

3

$3.3000000 \mathrm{E}+02$

$5.2800000 \mathrm{E}+02$

$5.1300000 \mathrm{E}+02$

$3.5400000 \mathrm{E}+02$

$6.7800000 \mathrm{E}+02$

$5.2800000 \mathrm{E}+02$

EXIT

EXIT

EXCHANGE

OUTPUT

CHIPLOT

FILE NAME

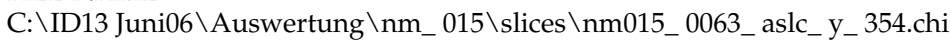

O.K.

EXCHANGE

$\%$ !* \Umbruch hier

DISPLAY

ARC SLICE

3

$3.3100000 \mathrm{E}+02$

$5.2800000 \mathrm{E}+02$

$5.1300000 \mathrm{E}+02$

$3.5500000 \mathrm{E}+02$

$6.7700000 \mathrm{E}+02$

$5.2800000 \mathrm{E}+02$

EXIT

EXIT

EXCHANGE

OUTPUT

CHIPLOT

FILE NAME

C: \ID13 Juni06 \Auswertung $\backslash n m_{-}$015 \slices $\backslash$ nm015_0063_aslc_y_355.chi O.K.

EXCHANGE

$\% !^{*} \backslash$ Umbruch hier

DISPLAY

ARC SLICE 
$3.3200000 \mathrm{E}+02$

$5.2800000 \mathrm{E}+02$

$5.1300000 \mathrm{E}+02$

$3.5600000 \mathrm{E}+02$

$6.7600000 \mathrm{E}+02$

$5.2800000 \mathrm{E}+02$

EXIT

EXIT

EXCHANGE

OUTPUT

CHIPLOT

FILE NAME

C: \ID13 Juni06 \Auswertung $\backslash n m \_015 \backslash$ slices $\backslash$ nm015_0063_aslc_y_356.chi

O.K.

EXCHANGE

$\% !^{*} \backslash$ Umbruch hier

DISPLAY

ARC SLICE

3

$3.3300000 \mathrm{E}+02$

$5.2800000 \mathrm{E}+02$

$5.1300000 \mathrm{E}+02$

$3.5700000 \mathrm{E}+02$

$6.7500000 \mathrm{E}+02$

$5.2800000 \mathrm{E}+02$

EXIT

EXIT

EXCHANGE

OUTPUT

CHIPLOT

FILE NAME

C: \ID13 Juni06 \Auswertung \nm_015\slices \nm015_0063_aslc_y_357.chi O.K

EXCHANGE

$\%$ !* $\backslash$ Umbruch hier

DISPLAY

ARC SLICE

$3.3400000 \mathrm{E}+02$

$5.2800000 \mathrm{E}+02$

$5.1300000 \mathrm{E}+02$

$3.5800000 \mathrm{E}+02$

$6.7400000 \mathrm{E}+02$

$5.2800000 \mathrm{E}+02$

EXIT

EXIT

EXCHANGE

OUTPUT

CHIPLOT

FILE NAME

C: \ID13 Juni06\Auswertung \nm_015 \slices \nm015_0063_aslc_y_358.chi

O.K.

EXCHANGE

$\%$ !* \Umbruch hier

DISPLAY

ARC SLICE

3

3.3500000E+02

$5.2800000 \mathrm{E}+02$

$5.1300000 \mathrm{E}+02$

$3.5900000 \mathrm{E}+02$

6.7300000E+02

$5.2800000 \mathrm{E}+02$

EXIT

EXIT

EXCHANGE

OUTPUT

CHIPLOT

FILE NAME

C: \ID13 Juni06\Auswertung \nm_015 \slices \nm015_0063_aslc_y_ 359.chi 
O.K.

EXCHANGE

$\% !^{*} \backslash$ Umbruch hier

DISPLAY

ARC SLICE

3

$3.3600000 \mathrm{E}+02$

$5.2800000 \mathrm{E}+02$

$5.1300000 \mathrm{E}+02$

$3.6000000 \mathrm{E}+02$

$6.7200000 \mathrm{E}+02$

$5.2800000 \mathrm{E}+02$

EXIT

EXIT

EXCHANGE

OUTPUT

CHIPLOT

FILE NAME

C: \ID13 Juni06 \Auswertung \nm_015\slices $\backslash n m 015 \_0063 \_$aslc_y_360.chi

O.K.

EXCHANGE

$\%$ !* $\backslash$ Umbruch hier

DISPLAY

ARC SLICE

3

$3.3700000 \mathrm{E}+02$

$5.2800000 \mathrm{E}+02$

$5.1300000 \mathrm{E}+02$

$3.6100000 \mathrm{E}+02$

$6.7100000 \mathrm{E}+02$

$5.2800000 \mathrm{E}+02$

EXIT

EXIT

EXCHANGE

OUTPUT

CHIPLOT

FILE NAME

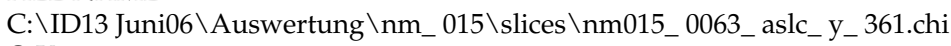

O.K.

EXCHANGE

$\% !^{*} \backslash$ Umbruch hier

DISPLAY

ARC SLICE

3

$3.3800000 \mathrm{E}+02$

$5.2800000 \mathrm{E}+02$

$5.1300000 \mathrm{E}+02$

$3.6200000 \mathrm{E}+02$

$6.7000000 \mathrm{E}+02$

$5.2800000 \mathrm{E}+02$

EXIT

EXIT

EXCHANGE

OUTPUT

CHIPLOT

FILE NAME

C: \ID13 Juni06 \Auswertung $\backslash n m_{-}$015 \slices $\backslash$ nm015_0063_aslc_y_362.chi

O.K.

EXCHANGE

EXIT

$\% !^{*} \backslash$ summation

FILE SERIES

SUMMATION

C: \ID13 Juni06 \Auswertung \nm_015 \slices $\backslash$ nm015_0063_aslc_y_352.chi YES

C: \ID13 Juni06 \Auswertung $\backslash n m_{-}$015 \slices $\backslash$ nm015_0063_aslc_y_362.chi

OUTPUT

CHIPLOT

FILE NAME

C: \ID13 Juni06 \Auswertung \nm_015 \nm015_0063_11aslc_ 1.chi 
O.K.

SUMMATION

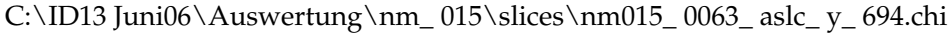
YES

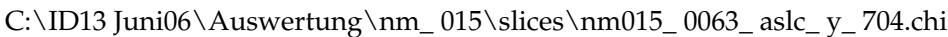

OUTPUT

CHIPLOT

FILE NAME

C: \ID13 Juni06 \Auswertung \nm_015 \nm015_0063_11aslc_2.chi

O.K.

SUMMATION

C: \ID13 Juni06 \Auswertung $\backslash n m_{-}$015 \slices $\backslash$ nm015_0063_hslc_y_523.chi YES

C: \ID13 Juni06 \Auswertung \nm_015\slices \nm015_0063_hslc_y_533.chi 1

OUTPUT

CHIPLOT

FILE NAME

$\mathrm{C}: \backslash$ ID13 Juni06 \Auswertung $\backslash n \mathrm{~nm} \_015 \backslash \mathrm{nm} 015 \_0063 \_11$ _hslc.chi

O.K.

SUMMATION

C: \ID13 Juni06 \Auswertung $\backslash n m_{-}$015 \slices $\backslash n m 015$ 0063_vslc_ x_508.chi YES

C: \ID13 Juni06 \Auswertung \nm_015 \slices $\backslash n m 015$ _0063_vslc_x_518.chi

OUTPUT

CHIPLOT

FILE NAME

C: \ID13 Juni06 \Auswertung \nm_015 \nm015_0063_11_vslc.chi

O.K.

$\% !^{*} \backslash$ Umbruch hier

EXIT

MACROS / LOG FILE

$\% ! * \backslash$ END OF IO MACRO FILE 


\section{C.2 Off-Axis Slices}

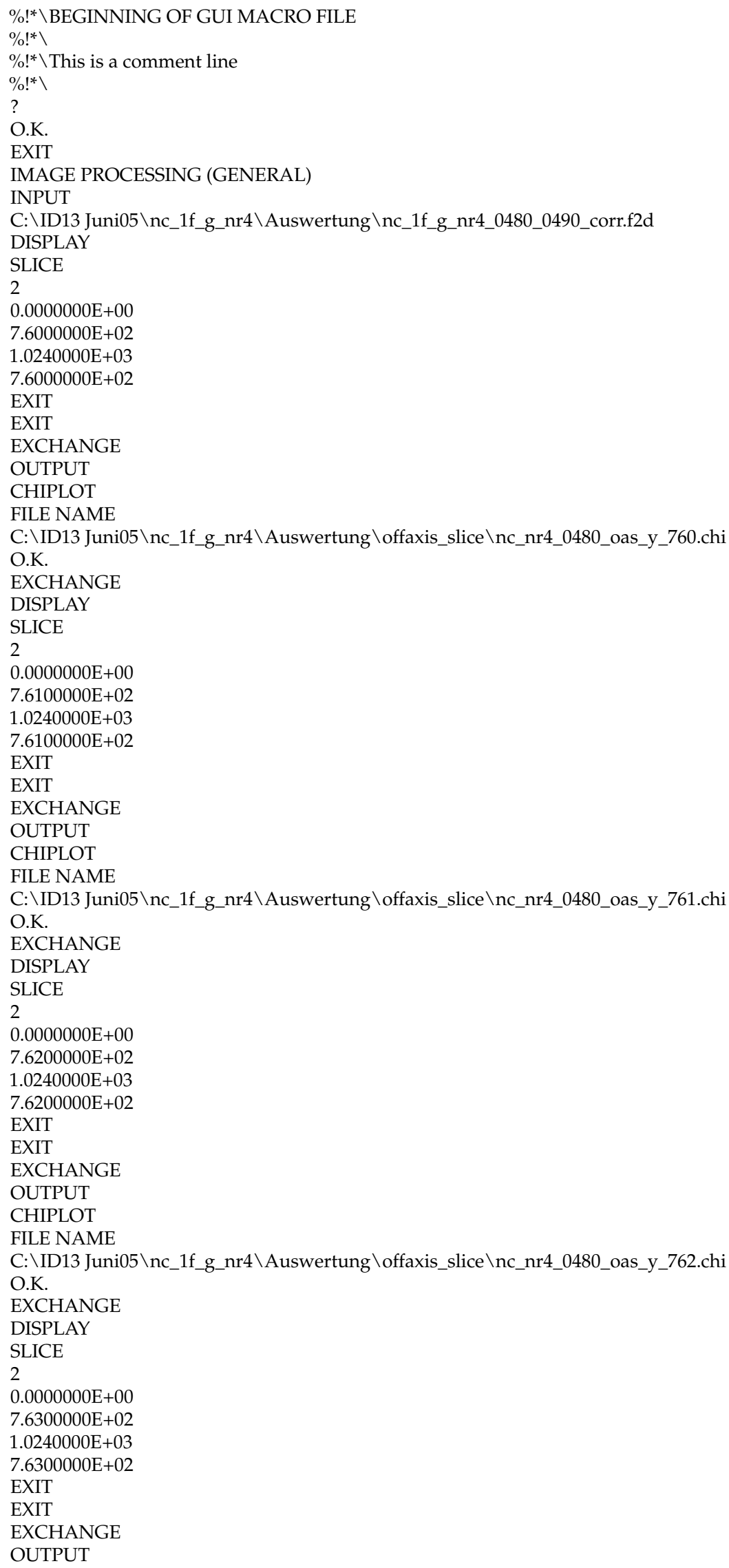


CHIPLOT

FILE NAME

C: \ID13 Juni05 \nc_1f_g_nr4\Auswertung \offaxis_slice \nc_nr4_0480_oas_y_763.chi

O.K.

EXCHANGE

DISPLAY

SLICE

2

$0.0000000 \mathrm{E}+00$

$7.6400000 \mathrm{E}+02$

$1.0240000 \mathrm{E}+03$

$7.6400000 \mathrm{E}+02$

EXIT

EXIT

EXCHANGE

OUTPUT

CHIPLOT

FILE NAME

C: \ID13 Juni05 \nc_1f_g_nr4\Auswertung \offaxis_slice \nc_nr4_0480_oas_y_764.chi

O.K.

EXCHANGE

DISPLAY

SLICE

2

$0.0000000 \mathrm{E}+00$

$7.6500000 \mathrm{E}+02$

$1.0240000 \mathrm{E}+03$

$7.6500000 \mathrm{E}+02$

EXIT

EXIT

EXCHANGE

OUTPUT

CHIPLOT

FILE NAME

C: \ID13 Juni05 \nc_1f_g_nr4\Auswertung \offaxis_slice \nc_nr4_0480_oas_y_765.chi

O.K.

EXCHANGE

DISPLAY

SLICE

$0.0000000 \mathrm{E}+00$

$7.6600000 \mathrm{E}+02$

$1.0240000 \mathrm{E}+03$

$7.6600000 \mathrm{E}+02$

EXIT

EXIT

EXCHANGE

OUTPUT

CHIPLOT

FILE NAME

C: \ID13 Juni05\nc_1f_g_nr4\Auswertung \offaxis_slice \nc_nr4_0480_oas_y_766.chi

O.K.

EXCHANGE

DISPLAY

SLICE

2

$0.0000000 \mathrm{E}+00$

$7.6700000 \mathrm{E}+02$

$1.0240000 \mathrm{E}+03$

$7.6700000 \mathrm{E}+02$

EXIT

EXIT

EXCHANGE

OUTPUT

CHIPLOT

FILE NAME

C: \ID13 Juni05 \nc_1f_g_nr4\Auswertung \offaxis_slice \nc_nr4_0480_oas_y_767.chi

O.K.

EXCHANGE

DISPLAY

SLICE 
$0.0000000 \mathrm{E}+00$

$7.6800000 \mathrm{E}+02$

$1.0240000 \mathrm{E}+03$

$7.6800000 \mathrm{E}+02$

EXIT

EXIT

EXCHANGE

OUTPUT

CHIPLOT

FILE NAME

C: \ID13 Juni05 \nc_1f_g_nr4\Auswertung \offaxis_slice \nc_nr4_0480_oas_y_768.chi

O.K.

EXCHANGE

DISPLAY

SLICE

$0.0000000 \mathrm{E}+00$

$7.6900000 \mathrm{E}+02$

$1.0240000 \mathrm{E}+03$

7.6900000E+02

EXIT

EXIT

EXCHANGE

OUTPUT

CHIPLOT

FILE NAME

C: $\backslash$ ID13 Juni05 \nc_1f_g_nr4\Auswertung \offaxis_slice \nc_nr4_0480_oas_y_769.chi

O.K

EXCHANGE

DISPLAY

SLICE

$0.0000000 \mathrm{E}+00$

$7.7000000 \mathrm{E}+02$

$1.0240000 \mathrm{E}+03$

$7.7000000 \mathrm{E}+02$

EXIT

EXIT

EXCHANGE

OUTPUT

CHIPLOT

FILE NAME

C: $\backslash$ ID13 Juni05 \nc_1f_g_nr4\Auswertung \offaxis_slice \nc_nr4_0480_oas_y_770.chi

O.K.

EXCHANGE

EXIT

FILE SERIES

SUMMATION

C: \ID13 Juni05\nc_1f_g_nr4\Auswertung \offaxis_slice \nc_nr4_0480_oas_y_760.chi YES

C: \ID13 Juni05 \nc_1f_g_nr4\Auswertung \offaxis_slice \nc_nr4_0480_oas_y_770.chi 1

OUTPUT

CHIPLOT

FILE NAME

C: \ID13 Juni05\nc_1f_g_nr4\Auswertung \offaxis_slice \nc_nr4_0480_0490_oaso.chi

O.K.

EXIT

IMAGE PROCESSING (GENERAL)

INPUT

C: \ID13 Juni05\nc_1f_g_nr4\Auswertung \nc_1f_g_nr4_0480_0490_corr.f2d

DISPLAY

SLICE

$0.0000000 \mathrm{E}+00$

$3.7600000 \mathrm{E}+02$

$1.0240000 \mathrm{E}+03$

$3.7600000 \mathrm{E}+02$

EXIT

EXIT

EXCHANGE 
OUTPUT

CHIPLOT

FILE NAME

C: \ID13 Juni05 \nc_1f_g_nr4\Auswertung \offaxis_slice \nc_nr4_0480_oas_y_376.chi

O.K.

EXCHANGE

DISPLAY

SLICE

2

$0.0000000 \mathrm{E}+00$

$3.7700000 \mathrm{E}+02$

$1.0240000 \mathrm{E}+03$

$3.7700000 \mathrm{E}+02$

EXIT

EXIT

EXCHANGE

OUTPUT

CHIPLOT

FILE NAME

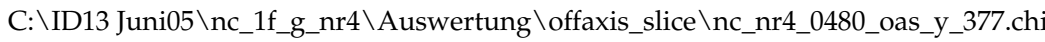

O.K.

EXCHANGE

DISPLAY

SLICE

2

$0.0000000 \mathrm{E}+00$

$3.7800000 \mathrm{E}+02$

$1.0240000 \mathrm{E}+03$

$3.7800000 \mathrm{E}+02$

EXIT

EXIT

EXCHANGE

OUTPUT

CHIPLOT

FILE NAME

C: \ID13 Juni05 \nc_1f_g_nr4\Auswertung \offaxis_slice \nc_nr4_0480_oas_y_378.chi

O.K.

EXCHANGE

DISPLAY

SLICE

2

$0.0000000 \mathrm{E}+00$

$3.7900000 \mathrm{E}+02$

$1.0240000 \mathrm{E}+03$

3.7900000E +02

EXIT

EXIT

EXCHANGE

OUTPUT

CHIPLOT

FILE NAME

C: \ID13 Juni05 \nc_1f_g_nr4\\Auswertung \offaxis_slice \nc_nr4_0480_oas_y_379.chi

O.K.

EXCHANGE

DISPLAY

SLICE

2

$0.0000000 \mathrm{E}+00$

$3.8000000 \mathrm{E}+02$

$1.0240000 \mathrm{E}+03$

$3.8000000 \mathrm{E}+02$

EXIT

EXIT

EXCHANGE

OUTPUT

CHIPLOT

FILE NAME

C: \ID13 Juni05 \nc_1f_g_nr4\\Auswertung \offaxis_slice \nc_nr4_0480_oas_y_380.chi

O.K.

EXCHANGE

DISPLAY 


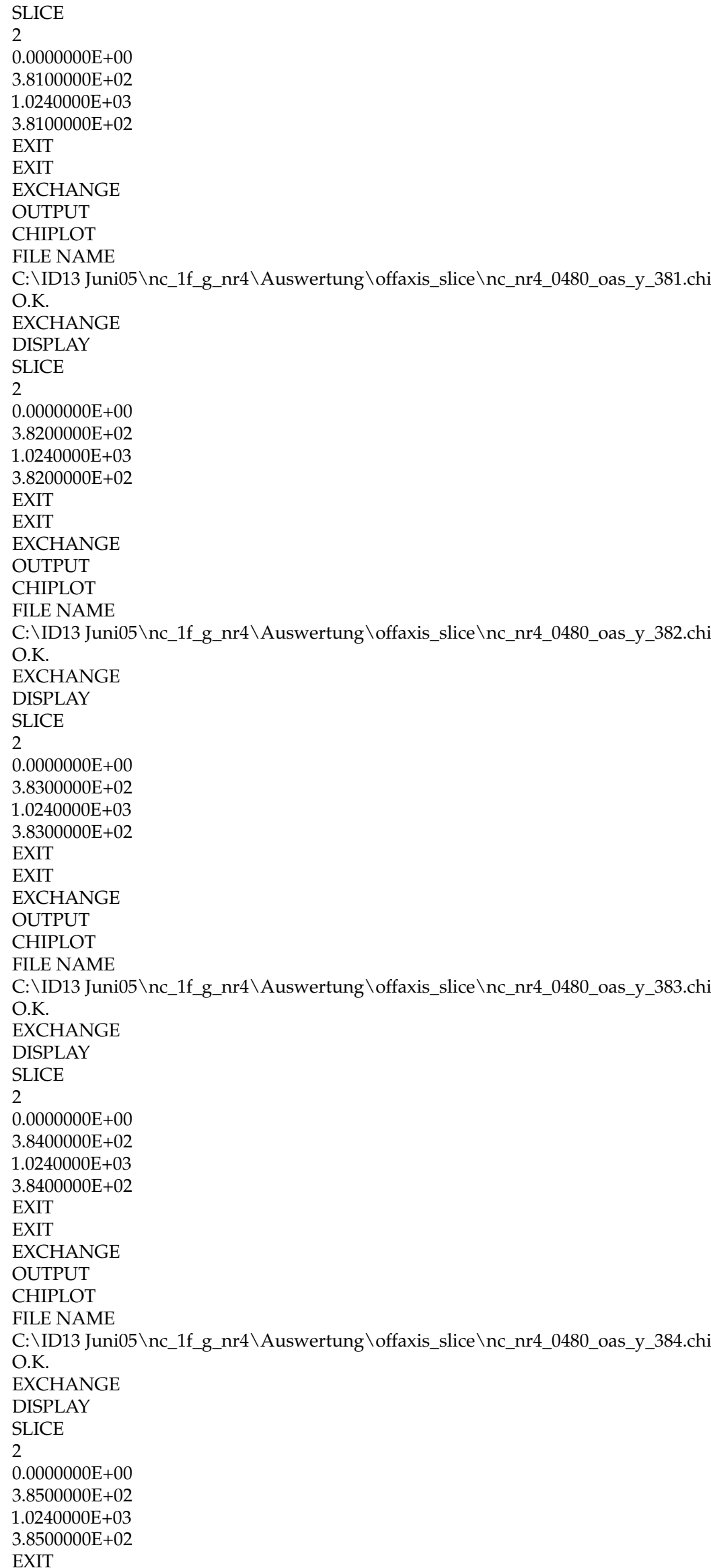


EXIT

EXCHANGE

OUTPUT

CHIPLOT

FILE NAME

C: \ID13 Juni05\nc_1f_g_nr4\Auswertung \offaxis_slice \nc_nr4_0480_oas_y_385.chi

O.K.

EXCHANGE

DISPLAY

SLICE

2

$0.0000000 \mathrm{E}+00$

$3.8600000 \mathrm{E}+02$

$1.0240000 \mathrm{E}+03$

$3.8600000 \mathrm{E}+02$

EXIT

EXIT

EXCHANGE

OUTPUT

CHIPLOT

FILE NAME

C: \ID13 Juni05 \nc_1f_g_nr4\Auswertung \offaxis_slice \nc_nr4_0480_oas_y_386.chi

O.K

EXCHANGE

EXIT

FILE SERIES

SUMMATION

C: \ID13 Juni05\nc_1f_g_nr4\\Auswertung \offaxis_slice \nc_nr4_0480_oas_y_376.chi YES

C: \ID13 Juni05 \nc_1f_g_nr4\Auswertung \offaxis_slice \nc_nr4_0480_oas_y_386.chi

OUTPUT

CHIPLOT

FILE NAME

C: \ID13 Juni05 \nc_1f_g_nr4\Auswertung \offaxis_slice \nc_nr4_0480_0490_oasu.chi

O.K.

EXIT

MACROS / LOG FILE

$\% ! * \backslash$ END OF IO MACRO FILE 


\section{C.3 Cake Integration}

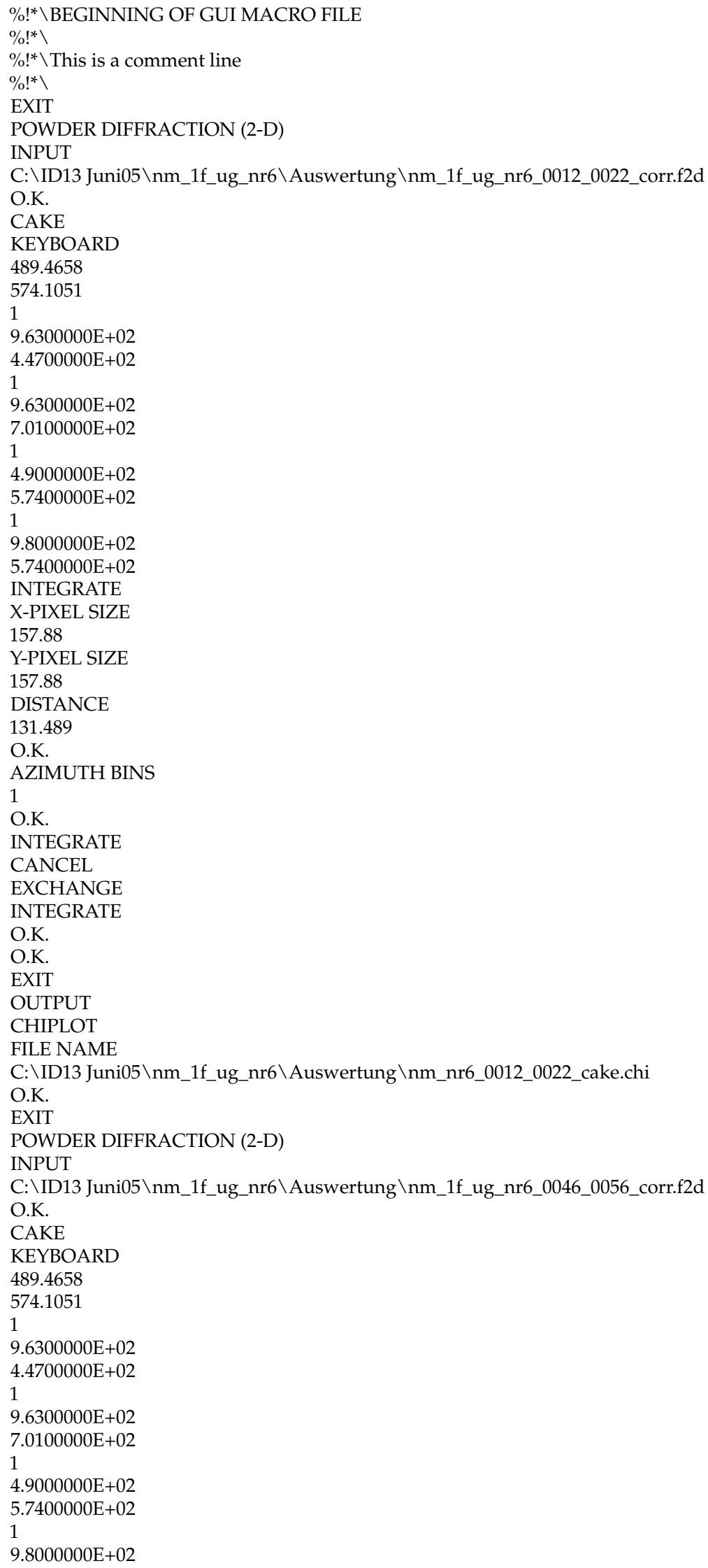




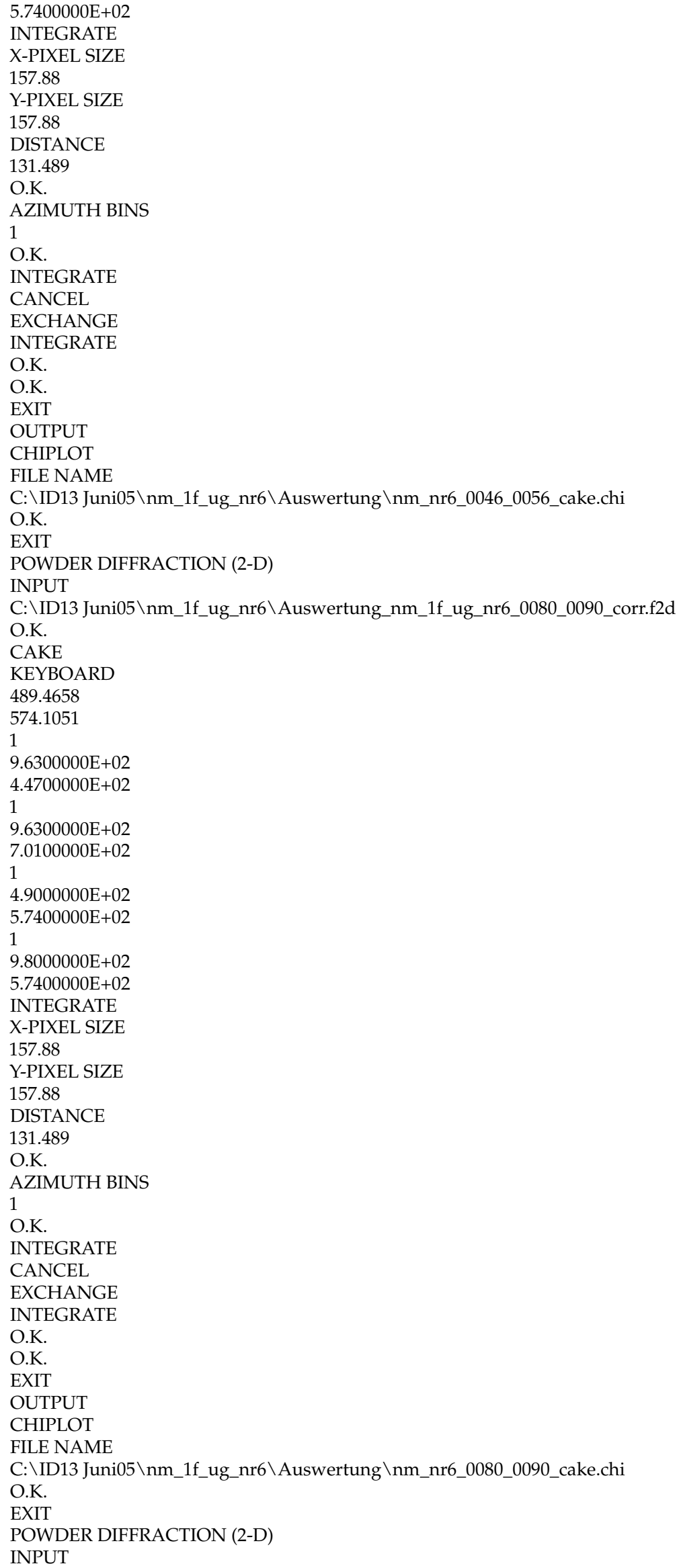


C: \ID13 Juni05 \nm_1f_ug_nr6\Auswertung \nm_1f_ug_nr6_0114_0124_corr.f2d

O.K.

CAKE

KEYBOARD

489.4658

574.1051

$9.6300000 \mathrm{E}+02$

$4.4700000 \mathrm{E}+02$

$9.6300000 \mathrm{E}+02$

$7.0100000 \mathrm{E}+02$

1

4.9000000E+02

$5.7400000 \mathrm{E}+02$

$9.8000000 \mathrm{E}+02$

$5.7400000 \mathrm{E}+02$

INTEGRATE

X-PIXEL SIZE

157.88

Y-PIXEL SIZE

157.88

DISTANCE

131.489

O.K.

AZIMUTH BINS

1

O.K.

INTEGRATE

CANCEL

EXCHANGE

INTEGRATE

O.K.

O.K.

EXIT

OUTPUT

CHIPLOT

FILE NAME

C:\ID13 Juni05 \nm_1f_ug_nr6\Auswertung \nm_nr6_0114_0124_cake.chi

O.K.

EXIT

POWDER DIFFRACTION (2-D)

INPUT

C: \ID13 Juni05\nm_1f_ug_nr6 \Auswertung \nm_1f_ug_nr6_0148_0158_corr.f2d

O.K.

CAKE

KEYBOARD

489.4658

574.1051

$9.6300000 \mathrm{E}+02$

$4.4700000 \mathrm{E}+02$

1

$9.6300000 \mathrm{E}+02$

$7.0100000 \mathrm{E}+02$

1

$4.9000000 \mathrm{E}+02$

$5.7400000 \mathrm{E}+02$

$9.8000000 \mathrm{E}+02$

$5.7400000 \mathrm{E}+02$

INTEGRATE

X-PIXEL SIZE

157.88

Y-PIXEL SIZE

157.88

DISTANCE

131.489

O.K.

AZIMUTH BINS 
1

O.K.

INTEGRATE

CANCEL

EXCHANGE

INTEGRATE

O.K.

O.K.

EXIT

OUTPUT

CHIPLOT

FILE NAME

C: \ID13 Juni05 \nm_1f_ug_nr6\Auswertung \nm_nr6_0148_0158_cake.chi

O.K.

EXIT

POWDER DIFFRACTION (2-D)

INPUT

C: \ID13 Juni05\nm_1f_ug_nr6 \Auswertung \nm_1f_ug_nr6_0182_0192_corr.f2d

O.K.

CAKE

KEYBOARD

489.4658

574.1051

1

$9.6300000 \mathrm{E}+02$

$4.4700000 \mathrm{E}+02$

1

$9.6300000 \mathrm{E}+02$

$7.0100000 \mathrm{E}+02$

$4.9000000 \mathrm{E}+02$

$5.7400000 \mathrm{E}+02$

$9.8000000 \mathrm{E}+02$

$5.7400000 \mathrm{E}+02$

INTEGRATE

X-PIXEL SIZE

157.88

Y-PIXEL SIZE

157.88

DISTANCE

131.489

O.K.

AZIMUTH BINS

1

O.K.

INTEGRATE

CANCEL

EXCHANGE

INTEGRATE

O.K.

O.K.

EXIT

OUTPUT

CHIPLOT

FILE NAME

C: \ID13 Juni05\nm_1f_ug_nr6 \Auswertung \nm_nr6_0182_0192_cake.chi

O.K.

EXIT

POWDER DIFFRACTION (2-D)

INPUT

C: \ID13 Juni05 \nm_1f_ug_nr6 \Auswertung \nm_1f_ug_nr6_0216_0226_corr.f2d

O.K.

CAKE

KEYBOARD

489.4658

574.1051

1

$9.6300000 \mathrm{E}+02$

$4.4700000 \mathrm{E}+02$

1 


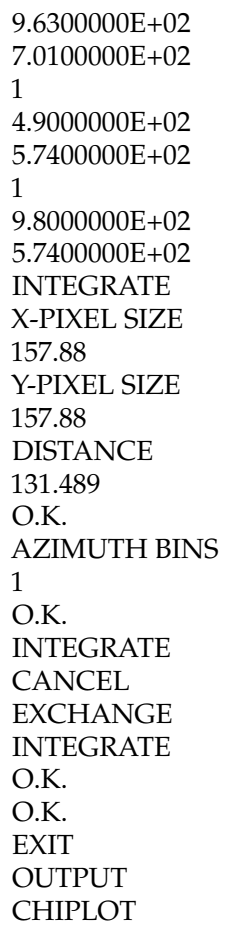


CHIPLOT

FILE NAME

C: \ID13 Juni05 \nm_1f_ug_nr6\Auswertung \nm_nr6_0250_0260_cake.chi

O.K.

EXIT

POWDER DIFFRACTION (2-D)

INPUT

C: \ID13 Juni05 \nm_1f_ug_nr6 \Auswertung \nm_1f_ug_nr6_0284_0294_corr.f2d

O.K.

CAKE

KEYBOARD

489.4658

574.1051

1

$9.6300000 \mathrm{E}+02$

$4.4700000 \mathrm{E}+02$

1

$9.6300000 \mathrm{E}+02$

$7.0100000 \mathrm{E}+02$

1

$4.9000000 \mathrm{E}+02$

$5.7400000 \mathrm{E}+02$

$9.8000000 \mathrm{E}+02$

$5.7400000 \mathrm{E}+02$

INTEGRATE

X-PIXEL SIZE

157.88

Y-PIXEL SIZE

157.88

DISTANCE

131.489

O.K.

AZIMUTH BINS

1

O.K.

INTEGRATE

CANCEL

EXCHANGE

INTEGRATE

O.K.

O.K.

EXIT

OUTPUT

CHIPLOT

FILE NAME

C:\ID13 Juni05 \nm_1f_ug_nr6\Auswertung \nm_nr6_0284_0294_cake.chi

O.K.

EXIT

POWDER DIFFRACTION (2-D)

INPUT

C: \ID13 Juni05\nm_1f_ug_nr6\Auswertung_nm_1f_ug_nr6_0318_0328_corr.f2d

O.K.

CAKE

KEYBOARD

489.4658

574.1051

1

$9.6300000 \mathrm{E}+02$

$4.4700000 \mathrm{E}+02$

1

$9.6300000 \mathrm{E}+02$

$7.0100000 \mathrm{E}+02$

1

$4.9000000 \mathrm{E}+02$

$5.7400000 \mathrm{E}+02$

1

$9.8000000 \mathrm{E}+02$

$5.7400000 \mathrm{E}+02$

INTEGRATE

X-PIXEL SIZE 
157.88

Y-PIXEL SIZE

157.88

DISTANCE

131.489

O.K.

AZIMUTH BINS

1

O.K.

INTEGRATE

CANCEL

EXCHANGE

INTEGRATE

O.K.

O.K.

EXIT

OUTPUT

CHIPLOT

FILE NAME

C:\ID13 Juni05 \nm_1f_ug_nr6\Auswertung \nm_nr6_0318_0328_cake.chi

O.K.

EXIT

MACROS / LOG FILE

$\%$ !* END OF IO MACRO FILE 


\section{Appendix D}

\section{List of Publications}

Anja Glišović, Richard J. Davies, Thorsten Vehoff and Tim Salditt Strain Dependent Structural Changes of Spider Dragline Silk submitted to Macromolecules

Anja Glišović and Tim Salditt Temperature Dependent Structure of Spider Silk by X-Ray Diffraction Applied Physics A, published online (2006)

Anja Glišović, Jürgen Thieme, Peter Guttmann and Tim Salditt Transmission X-Ray Microscopy of Spider Dragline Silk International Journal of Biological Macromolecules, Vol. 40, 87-95 (2007)

Thorsten Vehoff, Anja Glišović, Hauke Schollmeyer and Tim Salditt Mechanical Properties of Spider Dragline Silk: Humidity, Hysteresis and Relaxation submitted to Biophysical Journal

Arne Schäfer, Anja Glišović, Thorsten Vehoff and Tim Salditt Elasticity of Spider Dragline Silk - an AFM Study submitted to European Biophysical Journal 



\section{Appendix E}

\section{Acknowledgement}

I would like to thank Tim Salditt for this challenge and everything I've learned from him, as well as all members of the Institute für Röntgenphysik for the nice atmosphere. Furthermore special thanks to Thorsten Vehoff for the close and harmonious cooperation on spider silk and to our co-workers in the SFB 602 Annette Zippelius and Stefan Ulrich for fruitful discussions and cooperation, it was a pleasure to work with you.

I'd also like to thank Christoph Ollinger and Sebastian Kalbfleisch for their help with computer problems and Jochen Herbest, Thorsten Gronemann and Peter Nieschalk for the aid with technical issues.

Regarding individual beamtimes, I'd like to thank Richard J. Davies (ID13, ESRF), Ulrich Schade (BESSY II, Berlin), Manfred Rössle (EMBL, Hamburg) and Sergio Funari (DESY, Hamburg) for their support.

I also thank Prof. Dr. Friedrich G. Barth, Institut für Zoologie, Universität Wien and Prof. Michael E. Ellison, School of Textiles, Fiber and Polymer Science, Clemson University South Carolina who kindly provided spiders and silks.

I thank Hauke Schollmeyer, Jürgen Thieme, Michael Reese and Sebastian Panknin for proof-reading the manuscript or parts of it, as Dagmar Mirow for her help with spelling and grammar.

I also thank Klaus Giewekemeyer, Sebastian Aeffner and Sebastain Panknin for help during the beamtimes.

We gratefully acknowledge financial support by the Deutsche Forschungsgemeinschaft (DFG) through SFB 602/ B6.

Last but not least I want to thank my family who listened patiently to me moaning my problems, especially my godmother Carola Scholz, who was supportive of me and my family as long as she was with us.

Thank you all! 



\section{Lebenslauf}

\section{Persönliche Daten}

\begin{tabular}{l|l} 
Nachname & Glišović \\
Vorname & Anja \\
Geburtsdatum & 07.07.1978 \\
Geburtsort & Lübeck \\
Nationalität & deutsch
\end{tabular}

\section{Ausbildung}

\begin{tabular}{c|l} 
seit April 2005 & $\begin{array}{l}\text { Promotion am Institut für Röntgenphysik, Georg-August- } \\
\text { Universität Göttingen } \\
\text { wissenschaftliche Angestellte im II. Physikalischem Institut } \\
\text { Georg-August-Universität Göttingen }\end{array}$ \\
09.2000 - 04.2004 & $\begin{array}{l}\text { Physik Haupstudium an der Universität des Saarlandes Ab- } \\
\text { schluss: Diplom, Titel der Diplomarbeit "Röntgenstruktur- } \\
\text { analyse von Spinnenseide", extern angefertigt am Institut } \\
\text { für Röntgenphysik, Georg-August-Universität Göttingen } \\
\text { Physik Grundstudium an der Christian-Albrechts- } \\
\text { Universität Kiel Abschluss: Vordiplom }\end{array}$ \\
$09.1998-08.1998$ & $\begin{array}{l}\text { Oberschule zum Dom Lübeck, Abschluss: Allgemeine } \\
\text { Hochschulreife } \\
\text { Grund- und Hauptschule Grönauer Baum Lübeck }\end{array}$
\end{tabular}

